\title{
Anesthesia Recordkeeping: Accuracy of Recall with Computerized and Manual Entry Recordkeeping
}

Thomas Corey Davis

Virginia Commonwealth University

Follow this and additional works at: https://scholarscompass.vcu.edu/etd

Part of the Medicine and Health Sciences Commons

() The Author

\section{Downloaded from}

https://scholarscompass.vcu.edu/etd/2360

This Dissertation is brought to you for free and open access by the Graduate School at VCU Scholars Compass. It has been accepted for inclusion in Theses and Dissertations by an authorized administrator of VCU Scholars Compass. For more information, please contact libcompass@vcu.edu. 
C Thomas Corey Davis 2011

All Rights Reserved 


\section{ANESTHESIA RECORDKEEPING: ACCURACY OF RECALL WITH COMPUTERIZED AND MANUAL ENTRY RECORDKEEPING}

A Dissertation submitted in partial fulfillment of the requirements for the degree of $\mathrm{PhD}$ in Health Related Sciences at Virginia Commonwealth University.

by

THOMAS COREY DAVIS

Master of Science in Nurse Anesthesia, 1997

Bachelor of Science in Nursing, 1991

Director: DR. CHUCK BIDDLE, PHD

DIRECTOR OF RESEARCH, DEPARTMENT OF NURSE ANESTHESIA

AND

DR. JEFFERY A. GREEN, MD

VICE CHAIR, DEPARTMENT OF ANESTHESIOLOGY

Virginia Commonwealth University

Richmond, Virginia

May 2011 


\section{ACKNOWLEDGEMENTS}

"Not a sprint, but a marathon." Truer words have never been spoken regarding the process of writing a dissertation. Throughout this multi-year journey, I have been supported by my colleagues, friends and family. I would be remiss not to take a moment to recognize their efforts on my behalf.

To my nurse anesthesia colleagues, from both the clinical and educational arenas, I have never felt anything other than an outpouring of encouragement and pride in my endeavors. To work in an environment of mutual admiration and respect is too much to expect, and is a constant source of wonder to me. I am continuously amazed to discover those whom I hold in such high regard are so willing to assist me in the most menial of tasks to ensure my success. "Find a job you love, and you will never work a day in your life."

To my friends, I owe you repayment of many "rain checks," missed events, and postponed gatherings. Without exception, I have been supported and uplifted by brief words of encouragement and the factual dismissal of my excuses as I beg out of yet another social event. My journey is at an end, and yet is only beginning. I thank you for your understanding, and trust it will continue as I work to balance the demands of my career in academia with my small but essential core group of friends. I couldn't have done it without you. 
Finally, to my family, I owe you a debt I cannot repay. For countless evenings spent sequestered in my office, the sporting events and PTA meetings that went unnoticed, the ballet recitals attended with books open in my lap, I thank you for your understanding and support. To my long suffering wife, who cheerfully made excuses for my absences and shouldered the majority of responsibilities in order to give me time to complete this project, I am sincerely thankful. I have seen your love for me in each and every step you have taken so that I could address the demands of this research. I'll be home soon. To my children, who have grown older and taller since this journey began, I hope that my example has shown that great works require great determination and often sacrifice. Thank you for your loving support, and I hope you understand that Daddy's job is much more than just "homework." 
TABLE OF CONTENTS

Page

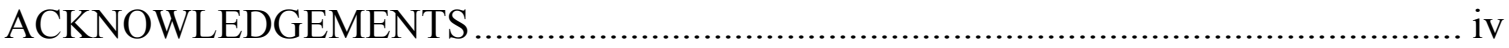

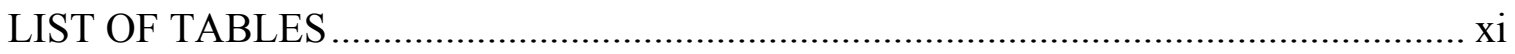

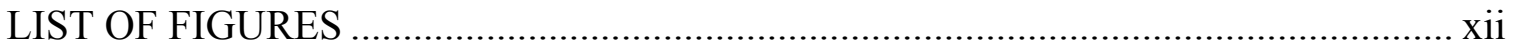

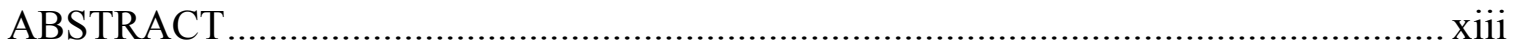

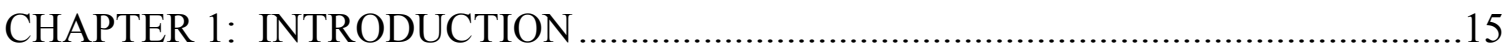

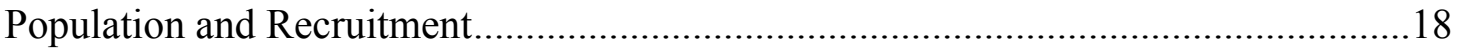

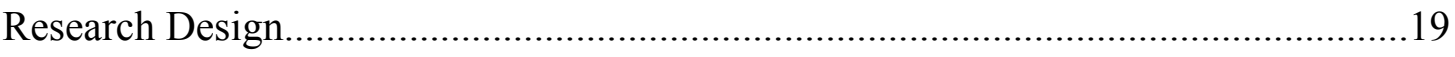

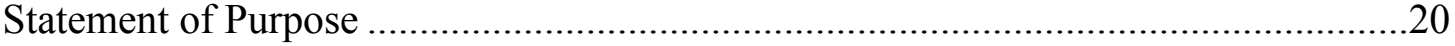

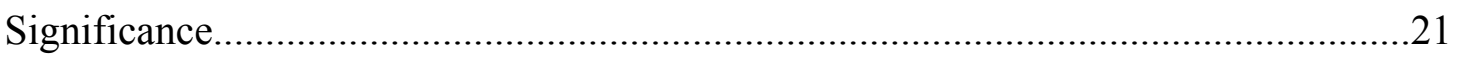

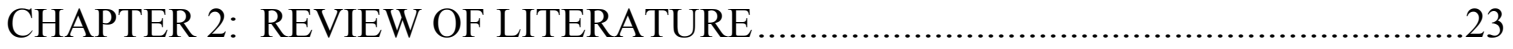

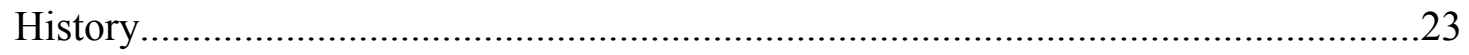

Early Development and Implementation ......................................................26

Standards of Monitoring and Voices of Caution ..................................................33

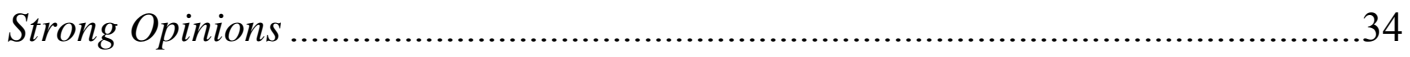

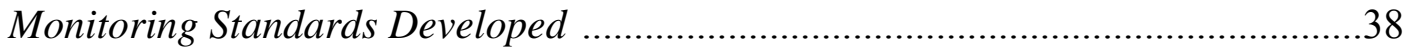

Advancement of Computing Technology ........................................................39

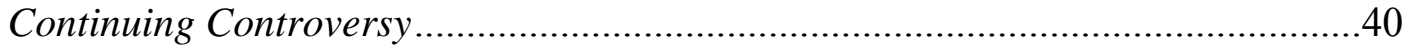

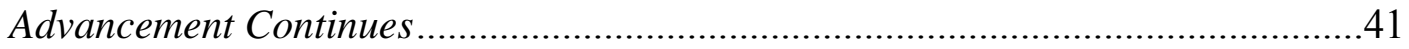


Experience with AIMS / Lessons Learned .............................................................43

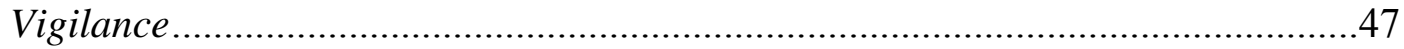

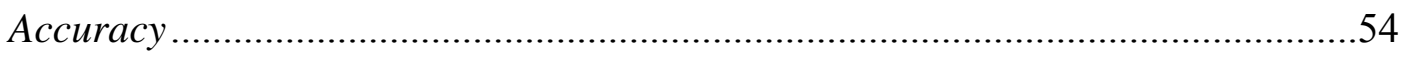

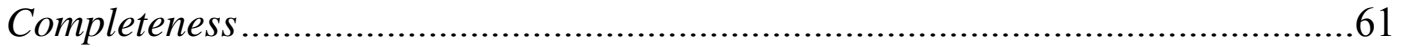

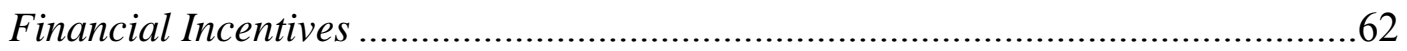

Enhanced Medico-legal Protection ....................................................................66

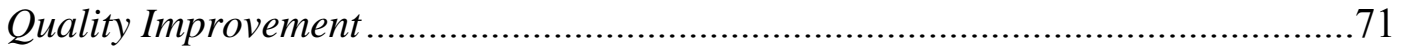

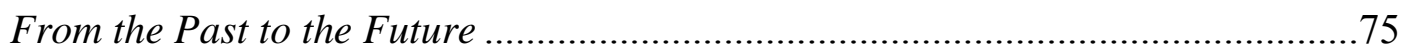

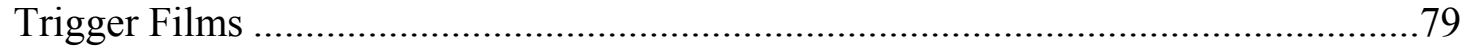

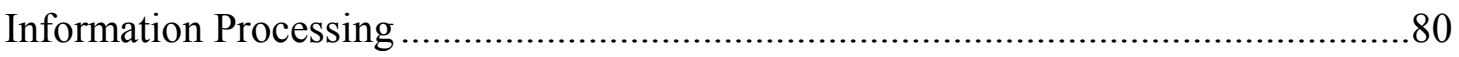

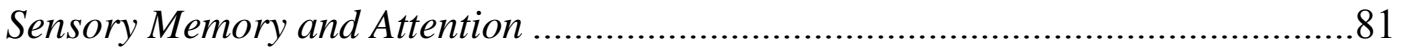

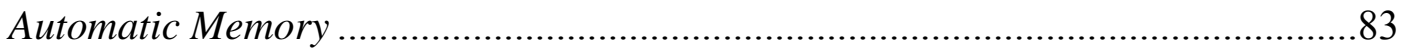

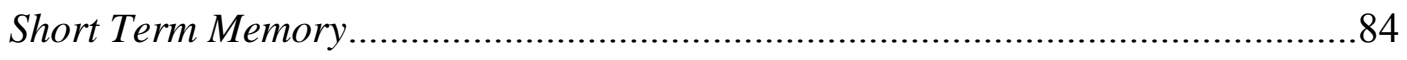

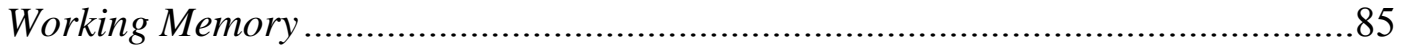

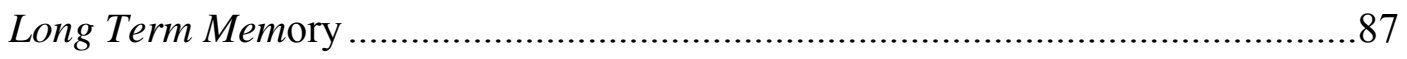

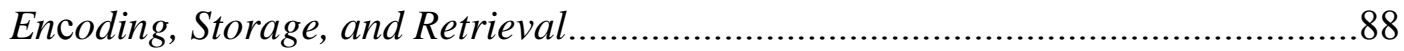

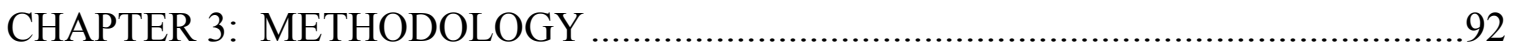

Relevance to Nurse Anesthesia..................................................................................93

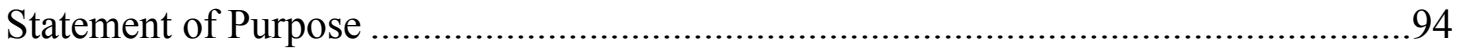

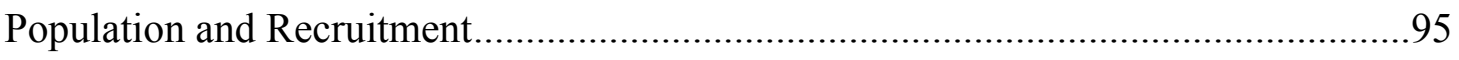

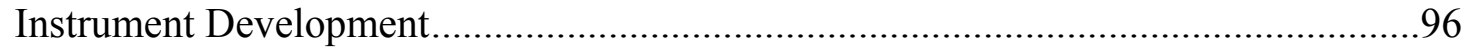




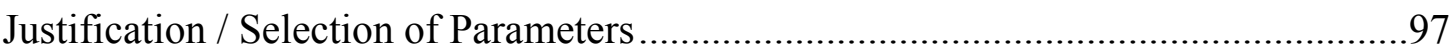

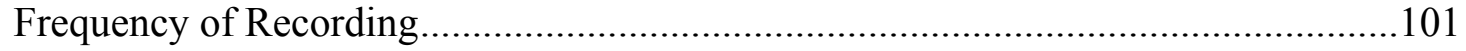

Measurement of Accuracy ............................................................................. 102

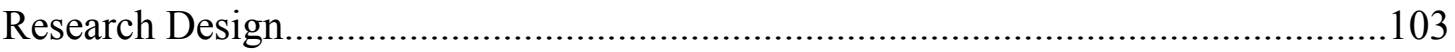

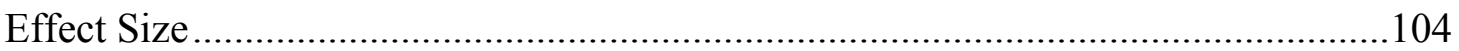

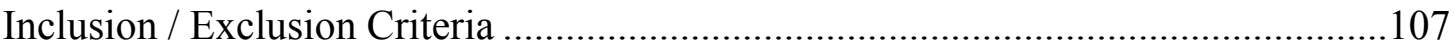

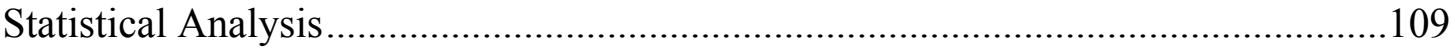

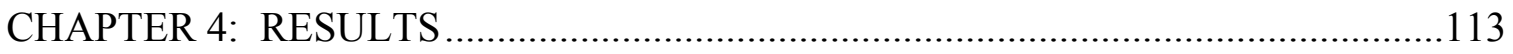

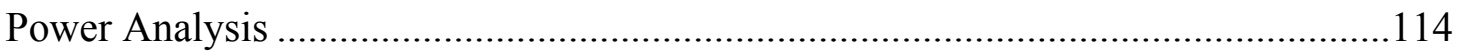

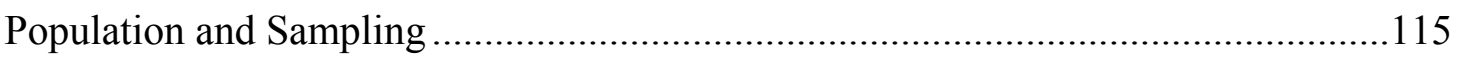

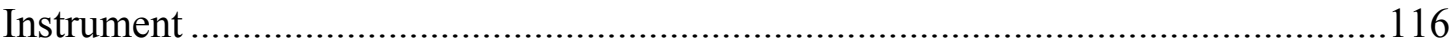

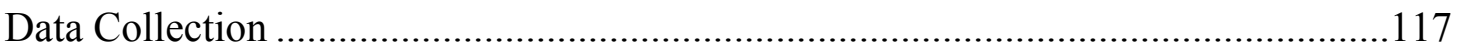

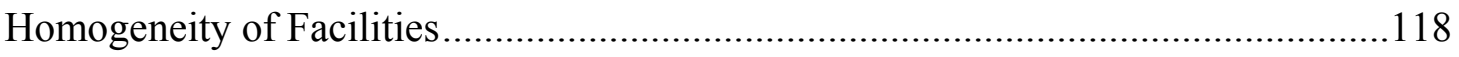

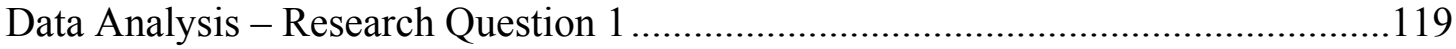

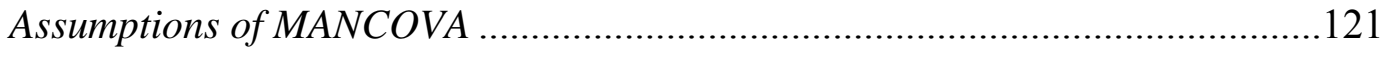

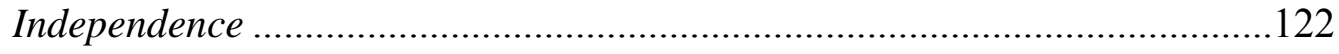

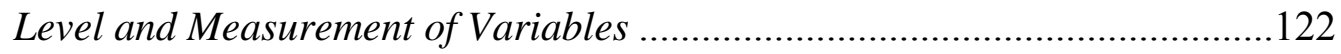

Linearity of Dependent Variables ......................................................... 124

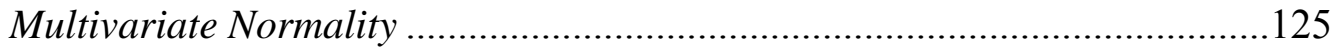

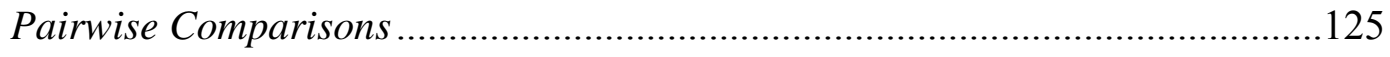

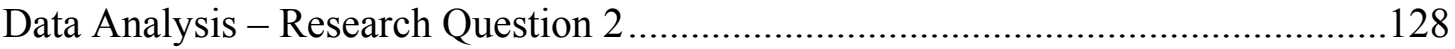




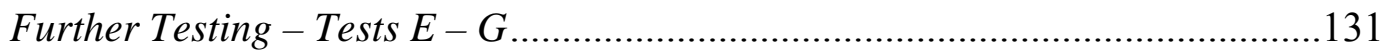

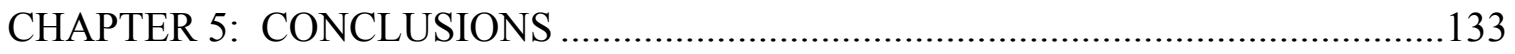

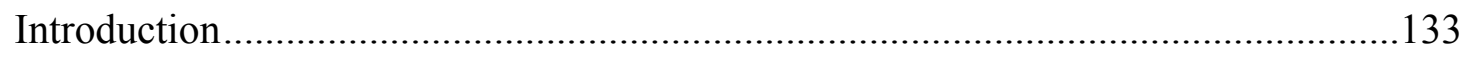

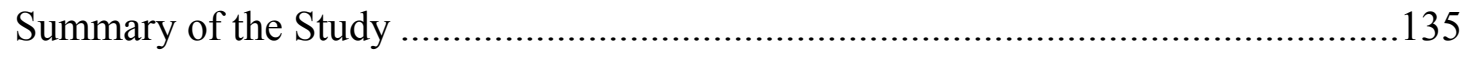

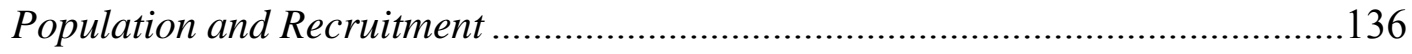

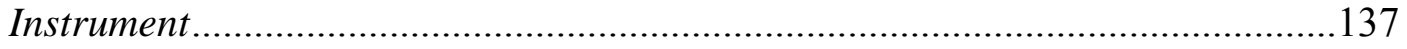

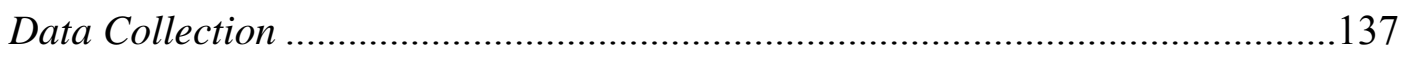

Overview of the Problem ...................................................................................... 138

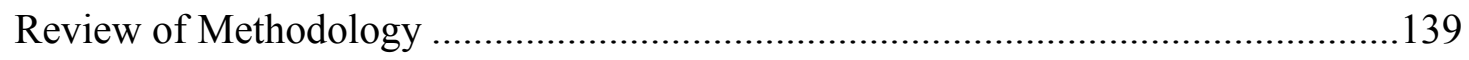

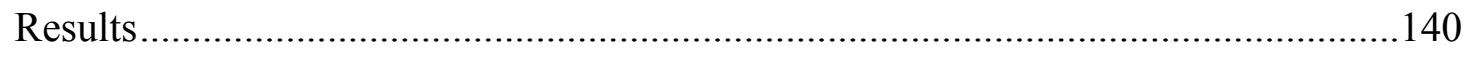

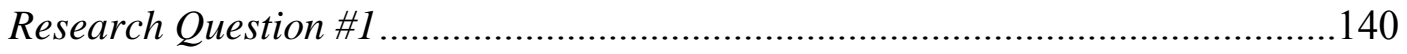

Research Question \#2............................................................................... 142

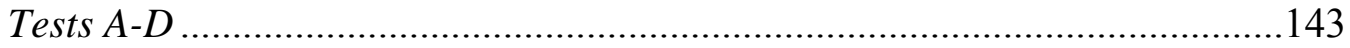

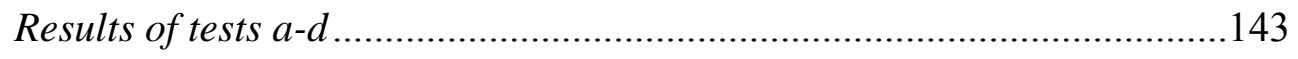

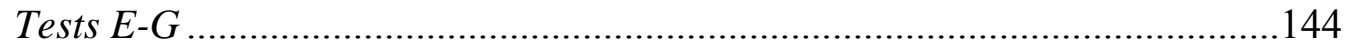

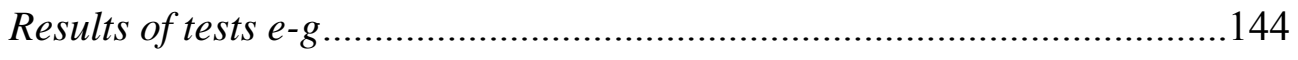

Relation of Results to the Literature ........................................................... 146

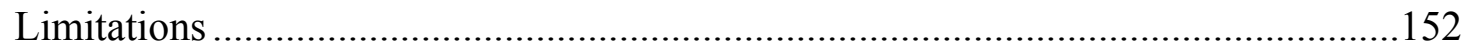

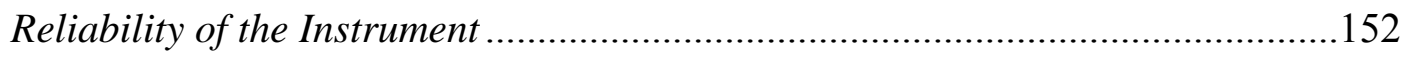

Threats to Internal and External Validity .......................................................152 


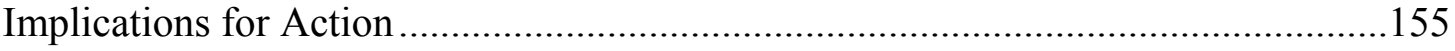

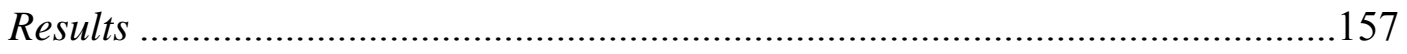

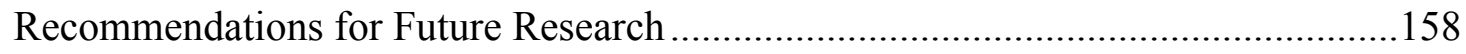

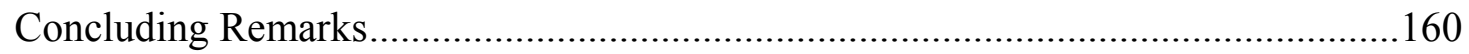

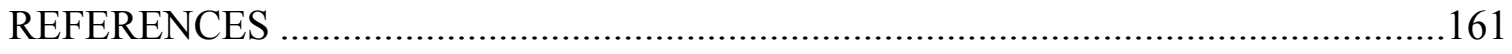

APPENDIX A：RECORDKEEPING SURVEY …………...................................179

APPENDIX B: SPEARMAN'S TEST OF CORRELATION OF COVARIATES ........181

APPENDIX C: HISTOGRAMS OF COVARIATES BY SITE......................................183

APPENDIX D: HISTOGRAMS OF COVARIATES BY METHOD OF

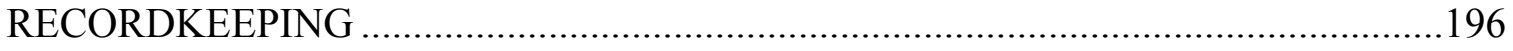

APPENDIX E: HISTOGRAMS OF DEPENDENT VARIABLES ………………........203

APPENDIX F: DATA ANALYSIS RESULTS - RESEARCH QUESTION \#2 ..........210

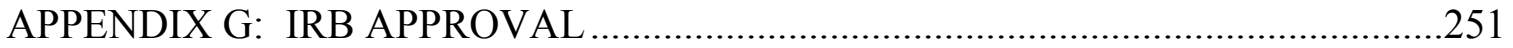

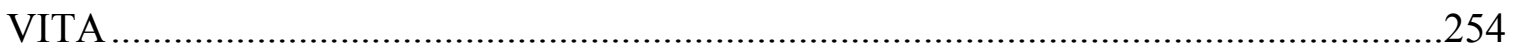




\section{LIST OF TABLES}

Table

Page

1: Facilities. .19

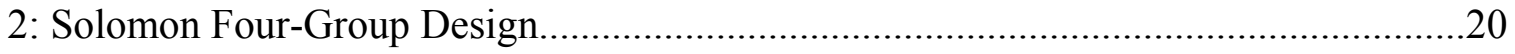

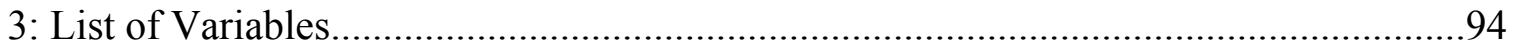

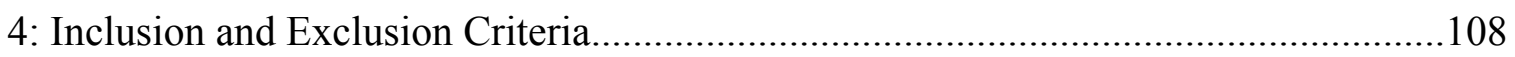

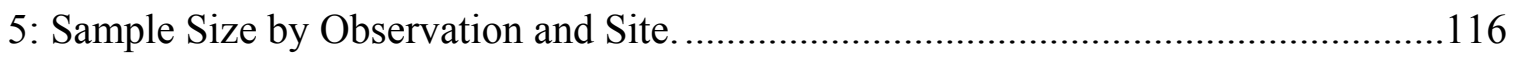

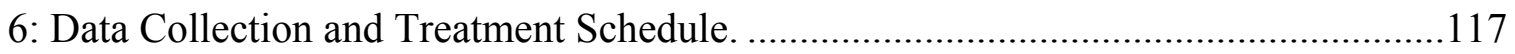

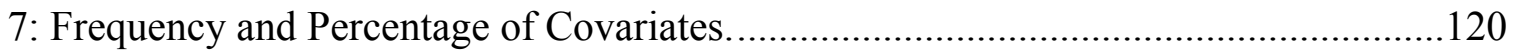

8: Levene's Test of Homogeneity of Variance of Covariates......................................123

9: Pillai's Trace - Homogeneity of VaPriance of Covariates and Site...........................124

10: Levene's Test of Homogeneity of Variance of Dependent Variables.......................125

11: Pairwise Comparisons - Dependent Variables by Method of Recordkeeping..........126

12: Pillai's Trace - Multivariate Tests of Method of Recordkeeping. ...........................127

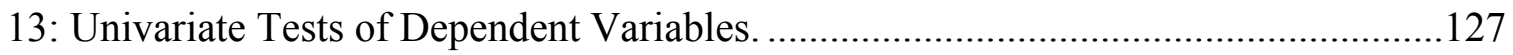

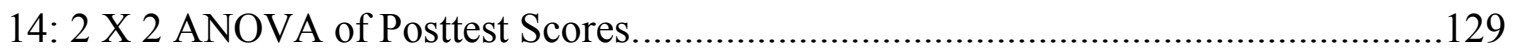

15: Two-way ANOVA of Pretest Data of Method of Recordkeeping..........................130 


\section{LIST OF FIGURES}

Figure

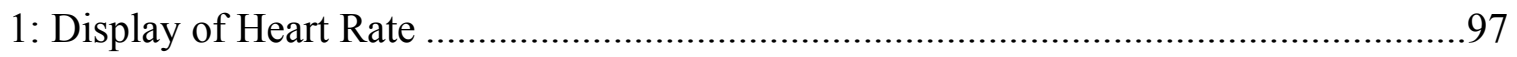

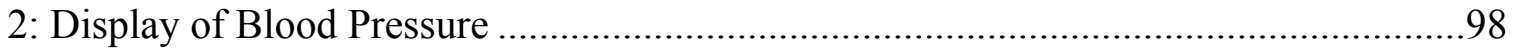

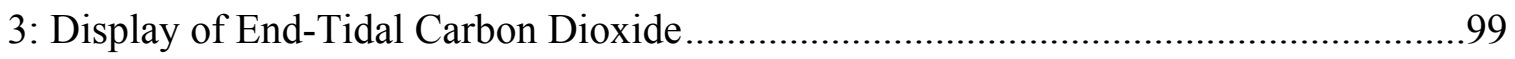

4: Display of Ventilation Screen - North American Dräger Fabius Model ....................100

5: Display of Inspiratory Pressure - North American Dräger Fabius Model ..................100 
ABSTRACT

ANESTHESIA RECORDKEEPING: ACCURACY OF RECALL WITH COMPUTERIZED

AND MANUAL ENTRY RECORDKEEPING

By Thomas Corey Davis, $\mathrm{PhD}$

A dissertation submitted in partial fulfillment of the requirements for the degree of $\mathrm{PhD}$ in Health Related Sciences at Virginia Commonwealth University.

Virginia Commonwealth University, 2011

Major Director: Dr. Chuck Biddle

Director of Research, Department of Nurse Anesthesia

And

Dr. Jeffery A. Green

Assistant Chief of Anesthesiology, Department of Anesthesia

Introduction: Anesthesia information management systems are rapidly gaining widespread acceptance. Aggressively promoted as an improvement to manual-entry recordkeeping systems in the areas of accuracy, quality improvement, billing and vigilance, these systems record all patient vital signs and parameters, providing a legible hard copy and permanent electronic record. At risk is a potential loss of "connectedness" to the patient with the use of computerized recordkeeping, perhaps jeopardizing vigilance.

Methods: This research analyzed differences in the accuracy of Certified Registered Nurse Anesthetists' (CRNAs) recall of specific patient variables during the course of an actual 
anesthetic case. CRNAs using computerized recordkeeping systems were compared to CRNAs using manual entry recordkeeping. Accuracy of recalled values of 10 patient variables was measured - highest and lowest heart rate, systolic blood pressure, inspiratory pressure, and endtidal carbon dioxide levels, lowest oxygen saturation and total fluid volume. In addition, a filmed educational vignette was presented to evaluate any effect on accuracy of recall following this presentation. Four tertiary care facilities participated in this research. A Solomon fourgroup research design was selected to control for the effect of pretesting on results of the filmed educational treatment.

Results: 214 subjects participated in this study; 106 in the computerized recordkeeping group, and 108 in the manual entry recordkeeping group. Demographic covariates were analyzed to ensure homogeneity between groups and facilities. No significant statistical differences were identified between the accuracy of recall among the groups. There was no statistically significant effect of the educational film vignette on accuracy of recall.

Conclusions: There was no difference in the accuracy of practitioners' recall of patient variables when using computerized or manual entry recordkeeping systems, suggesting little impact on vigilance. The educational film presented did not have an effect on accuracy of recall following the discussion of benefits and limitations of methods of recordkeeping. 


\section{CHAPTER ONE: INTRODUCTION}

In the United States, over 50 million anesthetics are delivered each year (Ishizawa, 2011). For each of these anesthetics, a detailed record is generated that includes vital signs, medications, and events of the surgery or procedure. Patients are monitored according to standards published by both the American Society of Anesthesiologists and the American Association of Nurse Anesthetists (AANA). These standards detail the vital signs and parameters that must be recorded by an anesthesia provider to document the monitoring of oxygenation, ventilation, circulation, and temperature (AANA - scope and standards for nurse anesthesia practice.2007; ASA, 2005).

The first known example of an anesthetic record can be found in the archives of the Massachusetts General Hospital, dated November 30, 1894 (Beecher, 1920). Developed by Dr. E. A. Codman, the record allowed the continuous documentation of heart rate, temperature, and respirations throughout the course of the anesthetic (Beecher, 1920). Systolic blood pressure readings were added to anesthesia records appearing after 1903 (Beecher, 1920). Both Dr. Codman and his contemporary, Dr. H.A. Cushing, indicated the merits of documentation of ether anesthetics, "It was undoubtedly a step toward improvement in what had been a very casual administration of a dangerous drug (Beecher, 1920)". 
The first known example of an automated anesthesia recordkeeping device dates to 1929 (McKesson, 1934). The device recorded three variables, oxygen percentage, respiratory volumes, and pulse pressure, on a continuous paper roll delineated in graphic form (McKesson, 1934). Fluctuations in these variables, primarily that of tidal respirations, indicated variations in the depth of anesthesia delivered, and any interruptions in the spontaneous breathing of the patient (McKesson, 1934). In his concluding remarks, Dr. McKesson indicated that such records would be "valuable for statistical study," much as modern anesthesia records are used for quality improvement (McKesson, 1934). Dr. McKesson also stated, "Automatic recording equipment is a more accurate means for the immediate determination of cause and effect during an administration. Such records stimulate closer observation and increase our knowledge of anesthesia, and should safeguard the patient (McKesson, 1934).”

Despite Dr. McKesson's praise, such means of recording data during an anesthetic did not take precedence over the manual entry chart. Only with the advent of computerized systems introduced in the 1970s is there further mention in the literature of electronic recordkeeping (Drui, Behm, \& Martin, 1973). With improvements in computing technology, efforts to automate medical records have attempted to ease the task of recordkeeping (Drui et al., 1973). Applications specific to the anesthesia record have begun to proliferate throughout the United States, due to established benefits of improved billing, legibility of the record, and access to data for quality assessment and improvement programs (Spring et al., 2007). In a recent survey of academic medical centers, $14 \%$ currently utilize an anesthesia information management system (AIMS), 
with an additional $29 \%$ in the planning or implementation phase (Egger Halbeis, Epstein, Macario, Pearl, \& Grunwald, 2008)

Proposed benefits over traditional manual entry recordkeeping systems (MERS), such as a reduction in workload or increased vigilance, have not been well established (Saunders, 1990). The proposed reduction in workload intended to increase the practitioners' time for monitoring of the surgical field or other activities of improved vigilance could also be spent in activities unrelated to direct patient care (Allard, Dzwonczyk, Yablok, Block, \& McDonald, 1995). With anesthesia reimbursement reductions and the steady advance of technology into all aspects of medical care, known benefits such as enhanced capture of billing services may result in the adoption of AIMS in many markets currently skeptical about their safety (Levitan, 2008).

AIMS are cited as superior to MERS in the areas of time management (Heinrichs, Monk, \& Eberle, 1997), data collection for quality improvement (Vigoda, Gencorelli, \& Lubarsky, 2006), and the capture of billing elements (Levitan, 2008). Concerns regarding the recording of artifact data as a potential source for malpractice claims (Feldman, 2004), excessive financial investment, increasing complexity of tasks, and decreasing attentiveness to the patient and monitors, thereby decreasing vigilance, (Abenstein, DeVos, Tarhan, \& Tarhan, 1992) have all been cited as limitations of AIMS. Studies analyzing vigilance and workload have failed to show significant differences between the two methods (Thrush, 1992). Examining the recall accuracy of specific vital signs, parameters, and events experienced by anesthesia practitioners utilizing AIMS and 
MER should serve as a starting point for further evaluation of the benefits and risks of recordkeeping systems.

\section{Population and Recruitment}

Certified registered nurse anesthetists (CRNAs) administer more than 30 million of the 50 million anesthetics delivered each year (American Association of Nurse Anesthetists, 2008). This study will draw a sample of CRNAs from a population of more than 200 CRNAs practicing at four medical centers across Virginia. CRNAs assigned to each facility on the day of evaluation will be asked to complete a simple questionnaire. A trained observer will provide each practitioner with brief relief from patient care, after constant attendance of the patient for a minimum of 30 minutes of anesthesia care during the maintenance phase of the case. The CRNA assigned to the case will be asked to turn away from the monitors and complete the questionnaire. The observer will document values directly from the trend data recorded in the patient monitor. To evaluate the accuracy of anesthesia providers' recall when recordkeeping with AIMS or MERS, a simple instrument has been developed (See Appendix I). Following establishment of face and content validity for this instrument, the research plan will be submitted for approval by the investigational review board (IRB) for Virginia Commonwealth University (VCU). With IRB approval, this instrument will be administered to practicing anesthesia providers participating in the actual delivery of anesthesia, and will require the provider to recall various aspects from the previous 30 minutes of their assigned case. Actual patient data will be collected by the relief CRNA to compare to the practitioners' recollections. Practitioners utilizing both AIMS and MERS will be evaluated, to allow 
comparison of any influence of the method of recordkeeping on the accuracy of practitioners' recall.

Four centers will be included in the project, selected by similarities of number of operating rooms, case load, and number of CRNA providers. These facilities are described in Table 1. All sites have agreed to participate in this research.

Table 1: Facilities

\begin{tabular}{ccccc}
\hline Facility & $\begin{array}{c}\text { Number of } \\
\text { Beds }\end{array}$ & $\begin{array}{c}\text { Number of } \\
\text { Operating } \\
\text { Rooms }\end{array}$ & $\begin{array}{c}\text { Number of } \\
\text { CRNAS }\end{array}$ & $\begin{array}{c}\text { Method of } \\
\text { Recordkeeping }\end{array}$ \\
\hline $\begin{array}{c}\text { Virginia } \\
\text { Commonwealth } \\
\text { University } \\
\text { Medical Center } \\
\text { (VCUMC) }\end{array}$ & 788 & 30 & 41 & AIMS \\
Inova Fairfax & 833 & 47 & 68 & AIMS \\
$\begin{array}{c}\text { Hospital (IFH) } \\
\text { Bon Secours St. }\end{array}$ & 369 & 30 & 41 & MERS \\
$\begin{array}{c}\text { Mary's Hospital } \\
\text { Medicorp Mary } \\
\text { Washington } \\
\text { Hospital }\end{array}$ & 412 & 26 & 51 & MERS \\
\hline
\end{tabular}

\section{Research Design}

A Solomon four group design has been selected for this study. This design minimizes the threat of testing on the outcome through the inclusion of one site that receives neither a pre-test, nor a treatment, but only a post-test. Each of the four facilities will be assigned to one of the four groups of this design as indicated in Table 2. Two sites will receive surveys as pre-tests, two sites will receive education with a trigger film (treatment), and all sites will be surveyed following the time of this presentation and again one month following the trigger film, as a post-test. 
Table 2: Solomon Four-Group Design

\begin{tabular}{lcccc}
\hline \multicolumn{1}{c}{ Group } & Survey & Trigger Film & Survey & $\begin{array}{c}\text { Survey- 1 month } \\
\text { Post - treatment }\end{array}$ \\
\hline St. Mary's (MERS) & $\mathrm{O}_{1}$ & $\mathrm{X}$ & $\mathrm{O}_{2}$ & $\mathrm{O}_{3}$ \\
Inova Fairfax (AIMS) & $\mathrm{O}_{4}$ & & $\mathrm{O}_{5}$ & $\mathrm{O}_{6}$ \\
VCUMC (AIMS) & & $\mathrm{X}$ & $\mathrm{O}_{7}$ & $\mathrm{O}_{8}$ \\
Mary Washington (MERS) & & & $\mathrm{O}_{9}$ & $\mathrm{O}_{10}$ \\
\hline
\end{tabular}

Statement of Purpose

The purpose of this research is to analyze the practitioner's accuracy in recalling patient data when using each method of recordkeeping. A second purpose of this research is to assess the effectiveness of a trigger film that details the benefits and limitations of both recordkeeping methods on the subsequent recall accuracy of anesthesia providers. This project seeks to answer two research questions:

- Do anesthesia providers using AIMS recordkeeping demonstrate equivalent recall accuracy of specific patient variables, as compared with anesthesia providers using a MERS?

- Does the instruction of the benefits and limitations of recordkeeping practices by trigger film influence the recall accuracy of specific patient variables by anesthesia providers using AIMS or MERS?

To answer these research questions, the following hypotheses will be analyzed:

$\mathrm{H}_{1} \quad$ For each anesthetic case, there will be no difference in the accuracy of recall of specific patient variables recorded by an anesthesia provider using AIMS or an anesthesia provider using a MERS.

$\mathrm{H}_{2} \quad$ For each anesthetic case, there will be no difference in the accuracy of recall of specific patient variables recorded by an anesthesia provider who 
has attended a trigger film presentation on the benefits and limitations of recordkeeping methods than a provider that has not received such instruction.

\section{Significance}

In the United States today, approximately $67 \%$ of all anesthetics delivered each year are administered by nurse anesthetists (American Association of Nurse Anesthetists, 2007). Since the initiation of anesthesia documentation, the majority of anesthesia providers have recorded this data manually on pre-printed forms. This form of MERS persists in more than 90 percent of anesthesia practices (Levitan, 2008), although the prevalence of AIMS is rapidly increasing (Egger Halbeis et al., 2008).

Improvements in the capture of billing elements, legibility of the record, and enhanced capabilities to review accurate data for quality improvement purposes have caused many experts in the field to brand the adoption of AIMS as inevitable (Levitan, 2008) (Hamilton, 1990) (Vigoda et al., 2006). In 2001, the Anesthesia Patient Safety Foundation published an initiative to utilize AIMS to improve patient safety (Cooper, 2007). This initiative was created in response to the Institute of Medicine's landmark report in 1999, which called for efforts to utilize developing technology to reduce the number of medical errors (Kohn, Corrigan, \& Donaldson, 1999). As recently as March 2008, an article in Anesthesiology News indicated that AIMS would "revolutionize anesthesia care" (Levitan, 2008).

Despite these claims, the safety of AIMS recordkeeping has not been established. Early in the debate, voices called for caution in their use (T. N. Noel, 1986)(Saunders, 
1990). Studies of vigilance that compare AIMS and MERS have been hindered by confounding variables and small sample size (Thrush, 1992). At the present time, AIMS are utilized by only five to ten percent of anesthesia providers (Levitan, 2008). This small percentage is cited to be due to the high initial cost of AIMS. With the increasing appeal for the implementation of AIMS (Levitan, 2008), there is an excellent opportunity for research into the benefits and limitations of each method of recordkeeping. Before widespread adoption of AIMS, researchers must examine differences in the "connectedness" of practitioners to the subtle trends of vital signs, parameters, and events that may influence patient safety. Researchers must also explore techniques to educate practitioners to enhance the benefits and minimize the limitations of either recordkeeping system. 


\title{
CHAPTER TWO: REVIEW OF LITERATURE
}

\author{
History
}

From the time of development of the first documented use of diethyl ether by Dr. William T.G. Morton in 1842, the safety of anesthesia administration has steadily increased. Initially the task of anesthetizing a patient was relegated to medical students under tutelage of a practicing surgeon, creating an anesthesia provider with a primary focus of studying the surgical procedure being preformed rather than vigilance toward the anesthetized patient. By the turn of the $20^{\text {th }}$ century, the morbidity and mortality of anesthesia delivery had become unacceptably high, and a provider dedicated to the specialty of anesthesia was sought (Gunn, 2005). At this time, physicians who specialized in anesthesia were few, due to the culture of medicine prevalent at the time. Surgeons recognized the need for anesthetists with specialized training, particularly those who would

"(1) be satisfied with the subordinate role that the work required, (2) make anesthesia their one absorbing interest, (3) not look on the situation of anesthetist as one that put them in a position to watch and learn from the surgeon's technic $\{\mathrm{sic}\},(4)$ accept the comparatively low pay, and (5) have the natural aptitude and intelligence to develop a high level of skill in providing the smooth anesthesia and relaxation that the surgeon demanded" (Thatcher, 1953) 
Most often, this role fell to nurses rather than physicians (Thatcher, 1953). Many of the physicians who administered anesthesia during this time were called in to service from the ranks of medical students studying the practice of surgery, were unskilled in the delivery of anesthetic agents, and often met with tragic results. A personal report shared by Dr. Harvey Cushing from the time of his medical training of a patient's death under anesthesia indicates not only the challenges of anesthesia delivery, but also the nonchalant attitude towards the mortality ascribed to such mortality by the surgeons at the time. Dr. Cushing was advised by the surgeon, "that sort of thing happed frequently and I had better forget about it and go on with the Medical School.” As a result of this and other such incidents, Dr. Cushing and his colleague, Dr. E.A. Codman of the Massachusetts General Hospital were encouraged to develop the first known examples of an anesthesia record. These documents recorded respirations, pulse rate, and temperature, along with narrative accounts of the events of the patients' reactions to the anesthetic. As stated by Dr. Cushing, "It was undoubtedly a step toward improvement in what had been a very casual administration of a dangerous drug. We do so much better with ether these days, but even so there remains much to learn" (Beecher, 1920).

More widespread use of handwritten accounts of anesthesia and surgery were to follow, but not for more than 20 years after these initial accounts. Even at this early date, voices of caution were raised, indicating that the manual documentation of "too elaborate a record of this kind might take the administrator's mind from his primary job". In defense, Dr. Cushing stated, "I feel most emphatically that it keeps his mind on his job" (Beecher, 1920). 
Perhaps as a result of similar concerns of distraction and reduced vigilance, in 1929, Dr. E.I. McKesson (1934) developed a device to record respiratory volumes, oxygen percentage, and pulse pressure, from which both the systolic and diastolic blood pressures were derived. Dr. McKesson (1934) advised, "It is very difficult for one person to count the pulse and respiration, measure the blood pressure and the volume of breathing, to determine the volume of rebreathing or the quantity of carbon dioxid $\{$ sic $\}$ used (the anesthetic), to note the dosage and a few other factors in their proper sequence and with sufficient frequency to aid in the administration". Dr. McKesson (1934) also indicates that these same limitations were raised as cause not to keep such a record of anesthesia, to better maintain vigilance focused toward the patient. Through examples of anesthetic records generated by his device, Dr. McKesson (1934) also indicates the first recorded incidence of "artifact" or erroneous data. Listed in Chart 2 a "notch" is indicated in the graphic display of respirations. Such a "notch" indicates a reduction in tidal volume of respirations, as with "deep narcosis (McKesson, 1934). In this example, this data is indicated to reflect a failure of the anesthetist to maintain an adequate mask seal to the patient's face, and this is indicated by a handwritten notation on the record. Dr. McKesson (1934) indicated that such records could be applied toward the ongoing research of anesthetic delivery techniques. He also indicated that such automatic recordkeeping had a higher degree of accuracy than handwritten records, many of which may be generated after the completion of the anesthetic rather than at the time of the event. Finally, the value of automatic records could be shown through improved 
knowledge of anesthesia by the practitioners, resulting in improved patient safety (McKesson, 1934).

While prophetic, Dr. McKesson's opinions and apparatus would not gain widespread use, and the handwritten record persisted with few changes into the distant future. Not until 1973 was there further mention of the benefits to accuracy in anesthesia recordkeeping that an electronic system could provide (Drui et al., 1973).

\section{Early Development and Implementation}

With the introduction in 1972 of a compact computerized calculator, the HP-35, the faithful slide-rule became instantly obsolete (Computer history museum - timeline of computer history. 2009). At this time of rapidly developing computing technology, Drui et al (1973) examined anesthesia practice with the intent of improving efficiency and, ultimately, patient care. The authors utilized "memomotion," a video imaging system that recorded data slower than actual time, to document the tasks of anesthesiologists. In addition, a trained observer with a stopwatch documented a series of twenty-four tasks, including periods of inactivity. Tasks were then rated according to the percentage of time devoted to the task, and the required knowledge, skill, and importance of each activity. The task of recordkeeping, while occupying a large proportion of the anesthesiologists' time, was determined to have very low requirements of knowledge, skill, and importance. The authors determined that such an activity of low importance, requiring a minimum of skill and knowledge to perform should be automated (Drui et al., 1973).

Five years later, computing technology had advanced to the point that such automation had become possible, with Zollinger et al (1977) providing a comparison 
between handwritten anesthesia records and those generated by "a computerized surveillance system"(Zollinger, Kreul, \& Schneider, 1977). Over this study of 100 patients, computer generated records were found to produce "acceptable blood pressure measurements 78 percent of the time," at a rate of one reading every 2.5 minutes. The resident anesthesiologists produced handwritten records that documented 94 percent of blood pressure measurements, at an interval of once every five minutes. Discrepancies between the two forms of recordkeeping occurred in $43 \%$ of the records, most of which occurred during times of high workload for the provider, such as during induction, or periods of time when the providers' attention was focused on other tasks. Some of the discrepancies were indicated, “...when the vital signs recorded by the anesthesiologist tended to make the record look smooth" (Zollinger et al., 1977). Zollinger et al (1977) concluded that the records produced were similar in accuracy, and advocated that computerization of the record could collect data at times when the provider was "otherwise occupied," and would eliminate data that was "underestimated by humans who tended to smooth out a record." Even at this early stage, voices of caution were raised, as the article included comments by a guest editor, calling into question the accuracy of the recording devices employed in the study, and cautioned against "a 'hands off' policy" (Zollinger et al., 1977).

A year later, Shaffer et al (1978) examined the prevalence of handwritten records and the potential use of these documents to improve the quality of anesthesia delivery. The authors cited a survey of 46 hospitals that revealed that as many as 8 percent did not maintain any form of anesthesia record, and that 17 percent did not record drug 
administration or dosages. This finding was supported by a similar study from Great Britain, finding that "25.9 percent of the anesthesia records were comprised of a single written entry, 45 percent of the anesthesiologists never analyzed their records, and 51.6 percent analyzed them only sporadically" (Shaffer, Kaiser, Klingenmaier, \& Gordon, 1978). Further analysis of the methods of recordkeeping indicated that many limitations of production of these documents existed. These limitations included the difficulties with production of copies, limited space available for documentation, incorrect or omission of entries, and illegibility of handwriting. These limitations indicated a need for greater automation of the anesthetic record, to improve the current deficiencies and reduce the anesthetist's time spent on the task of recordkeeping. Shaffer et al (1978) surveyed anesthesiologists at their facility to devise a list of attributes for an ideal automated recordkeeping system. Shaffer et al (1978) summarized the survey into four main attributes deemed necessary by over $90 \%$ of respondents:

1. Automatic capture of information with the ability to edit inaccurate or erroneous data.

2. Ability to enter data manually through a keyboard, light pen, or graphic display.

3. Reliability of function

4. Ease of electronic storage and retrieval of information

Shaffer et al (1978) concluded that a "semiautomated" system would be the solution to the limitations of the system and account for the suggested improvements. Such a system would maintain many aspects of the handwritten record, to allow for manual entry and 
error correction, while providing automated capture of vital signs, allowing for a savings of 33 to $50 \%$ of the time spent producing a record by hand.

At this time in history, standardization of anesthetic equipment was in its infancy. The Pin-Index system, a method to prevent errors of connecting incorrect gas cylinders to an anesthesia machine, had only recently been introduced (Thompson, 1978). The automated non-invasive blood pressure device manufactured by Applied Medical Research, Tampa Florida, had just been introduced to the field, and was beginning to appear in clinical anesthetizing locations. This device, the Dinamap, provided an automated blood pressure reading with reliability, but provided only a visual display, and not an automated record of readings (Lindop, 1981). Against this background of developing technology, Apple et al (1982) offered a proposal for the development of a semiautomatic recordkeeping system similar to that called for by Shaffer et al in 1978. Apple et al (1982) provides details of the Abbograph, from Abbot Labs in Houston, Texas. The Abbograph provided only vital signs on a graphic plotting device, and had no capability for manual entry, an attribute deemed essential by both research studies. The authors offered a system of their own design, capable of both automatic capture of vital signs, as well as manual entry through a keypad of seven categories of data, including "anesthetic gases, intravenous fluids, body fluid losses, blood pressure data, ventilator settings, general patient record information and general events." Entry of data into this system could be made at the time of the event, at a later time, or "time independent," for patient information that is not time sensitive. To evaluate the device, the authors compared 20 handwritten records and 20 keypad records. Of the entries made by hand, 
$77.4 \%$ were discovered to be correct, compared to $93.1 \%$ accurate entries made with the keypad device, with enhanced legibility. The keypad device, however, omitted some blood pressure readings which the handwritten records did not. Handwritten records of blood pressure were often either incorrect or entered at incorrect times, indicating that the anesthetist entered this data from memory at some later time (Apple, Schneider, \& Fadel, 1982).

Mitchell (1982) added to the call for automation of the anesthetic record, surmising that an automated record would improve accuracy, enhance legibility, decrease the time spent on documentation, and still produce a more complete record. In addition, such a record could then be electronically stored, with data then mined for the purposes of improvement of quality and patient safety. Mitchell (1982) illustrated that the technology necessary for an automated system was currently available, but graphic, full color displays and the need for a printed hard copy remained financial obstacles to adoption. A focus on ease of user interface with the device was listed as a priority for full acceptance by those practitioners who would use the device, and failure of this aspect of design could preclude adoption of even the most sophisticated device (Mitchell, 1982). Mitchell (1982) summarized that widespread adoption of automated anesthesia recordkeeping devices would be dependent upon the resolution of the problems of inexpensive high fidelity color displays and printers, greatly improved user interfacing systems, fully integrated alarm systems, enhanced capability for data storage and retrieval, and overall system reliability. 
In 1985, Block et al published an account of their efforts to design and implement an automated anesthesia recordkeeping device at Duke University, in Durham, North Carolina. This system, the "Duke Automatic Monitoring Equipment" or DAME, was designed and implementation was attempted but thwarted due to several technical attributes. The DAME was designed to capture electrocardiograph (ECG), electroencephalomyogram (EEG), finger pulsimeter, temperature, and invasive blood pressure inputs. User interface was accomplished through a lightpen and a series of bar codes for various commands and entries into the system, with the bar codes contained in plastic coated pages located on the top of the DAME cart. A graphic display of ECG, EEG, and pulsimetry was provided by a cathode-ray tube (CRT) in real time, with an alarm panel with auditory and lighted alarms keyed to each monitoring variable. Data was collected by each DAME device, and then transferred to a larger computer system housed in a separate building for electronic storage. Introduction of the device was accomplished through the efforts of both the system design team as well as members of the maintenance and engineering faculty at the hospital, educating those who would be utilizing the device and providing a detailed instruction manual (F. E. Block Jr. et al., 1985).

Upon introduction, many unexpected problems with the DAME system arose, despite extensive testing and evaluation. Block et al (1985) cited the cumbersome size of the device, which was difficult for one person to move, despite a wheeled cart design, as a significant limitation to its acceptance. Connecting cables were easily damaged, and were difficult to connect from the patient to the machine. The bar code user interface 
proved especially problematic, in unexpected ways. Adjustments to the graphic display on the CRT could be made through the bar code system, but required multiple scans of the same bar code; producing such small changes that many providers attributed the lack of perceptible change to malfunction. A bar code for "declare medical emergency" was tested by practitioners so frequently that these messages were rapidly ignored, and this bar code was subsequently removed. Much of the data intended to be captured by the DAME system was found to be omitted, or entered incorrectly. Practitioners, to enhance time savings, frequently entered erroneous data for patient identifiers, such as a medical record of "111111," which produced multiple copies of the same medical record containing data from multiple cases, rendering any attempt at data retrieval erroneous, if not impossible. Of more than 20,000 printed records, Block et al (1985) stated that less than 50 were considered to be complete documents. Faced with these many limitations, many practitioners used the device as a monitor for vital sign monitoring only; most simply refused to use the device. Attempts to reduce the size of the device resulted in the creation of the MicroDAME, a much smaller version of the original device. Prior to introduction of this smaller device, all funding was eliminated, halting further progress. In retrospect, Block et al (1985) stated,

We believe that the human factor remains the greatest barrier to the computerized operating room monitor. Certainly, new monitoring equipment should not be introduced simultaneously with other operating room changes. Even under the best of circumstances, however, many anesthesia personnel cannot be expected to learn how to properly use any 
new monitor. They will always make mistakes and fail to make necessary adjustment. It cannot be assumed that they will interact with the monitor in a useful way. They will monitor only those variables they want to monitor. Rather than a complex monitor, they want a simple one. Perhaps they are right in their preference. Yet, a monitor can be simple on the outside, complex on the inside, and provide the necessary information for patient care.

Despite the many, unexpected limitations, Block et al (1985) had shown that it was possible to electronically capture all aspects of the complex art and science of anesthesia care. Many more attempts would follow.

\section{Standards of Monitoring and Voices of Caution}

In 1985 , a sweeping change to the practice of anesthesia occurred with the publication of "Standards for patient monitoring during anesthesia at Harvard Medical School" (Eichhorn et al., 1986). For the first time, guidelines were suggested for the uniform monitoring of patients, as well as suggestions for the frequency of recording vital signs and other events of anesthesia and surgery. Within months, the American Society of Anesthesiologists (ASA) approved and published similar standards for basic monitoring of patient receiving anesthetic care (ASA House of Delegates, 2005). As if in answer to the lamentations of Block et al (1985), these standards set specific guidelines for which vital signs, parameters, and other aspects of anesthesia delivery should be monitored, and how frequently these values should be recorded. These standards also offered practitioners the option to waive various elements of monitoring, in extenuating 
circumstances such as emergency or trauma, but stated, "...it is recommended that when this is done, it should be so stated (including the reasons) in a note in the patient's medical record" (ASA House of Delegates, 2005). Such a statement provided for realistic options for practitioners to maintain safe practice while still exercising their own clinical judgment regarding the feasibility of monitoring according to the standards. Similar standards were also instituted by the American Association of Nurse Anesthetists (AANA) (AANA - scope and standards for nurse anesthesia practice. 2007). Widespread adoption of these standards swept the United States, and these same standards, with revisions for technological advancements, continue to this day and apply to all patients receiving any form of anesthesia, in any location or setting.

Also in 1985, Rosen and Rosenzweig wrote a letter to the editor of Anesthesiology, renewing the call for computerized recordkeeping of anesthesia practice. The letter provided suggestions for the development of a simple recording device that could be created with publicly available computing devices, printers, and software (Rosen \& Rosenzweig, 1985a). This short note to the editor set off a storm of controversy which was to direct the focus of much research for years to come.

\section{Strong Opinions}

In a letter to the editor, Rosen and Rosenzweig (1985) described the design of a computerized anesthesia recordkeeping device. This device was created from readily available consumer technology, retained a compact design, and allowed either manual or automatic entry of vital signs. Many of the suggestions from Drui et al (1973) were incorporated, including the ability to enter data in real time or asynchronously, as well as 
the ability to edit data that was automatically captured. Data from the computer was then sent to a "plotter" printer kept outside the operating room, eliminating noise (Rosen \& Rosenzweig, 1985a). An example of the hardcopy produced by this system was also provided by the authors. Software for the device was also described as commercially available, and specific information was provided for interested parties. Rosen and Rosenzweig (1985) stated that, despite the lack of definitive research, a "more legible and accurate record is a better defense in malpractice suits."

In response, Lees (1985) wrote to the editor in the next issue, advising caution. Lees (1985) stated, "To date no pursuit in anesthesiology technology has claimed more and delivered less than the search for a "computerized anesthesia record." The author went on to illustrate inconsistencies in the suggestions made by Rosen and Rosenweig (1985), including the ease of manual data entry and automatic capture of vital signs. In reference to this automatic documentation, Lees (1985) indicated that, "This requires special communications software. It's not enough that the plugs match!" Communication between electronic devices was cited as a significant obstacle to implementation of such a system, a problem that has only begun to be resolved with the advent of more modern systems today (Levitan, 2008). Lees (1985) called into question the lack of mention of the time required to print the documents described, indicating that this factor alone could be a significant hindrance to the widespread adoption of computerized recordkeeping. Accuracy was also questioned, as simply reporting information accurately does not indicate that the data recorded is correct. "Garbage in, garbage, out," Lees (1985) stated, advising that automated records were only as accurate as the monitoring devices that 
produced the readings to be documented. Finally, Lees (1985) disagreed with the opinion that increased legibility of the record would reduce malpractice claims or would support the position of the anesthesia team in malpractice claims. He stated, "The inference that somehow or other by using this magic box a successful defense is mounted to malpractice litigation is completely unsubstantiated" (Lees, 1985).

Rosen and Rosenweig (1985) responded to these claims, and offered to "dispel some of these misconceptions." In support of the claim that automatic capture of vital signs could be easily obtained, the authors cite standards of the Electronic Industry Association that would regulate the format of data and frequencies of signals to allow different devices to transfer information (Rosen \& Rosenzweig, 1985b). In truth, such standards did not create the ease of data transfer the authors described, as only recently have advancements been created to allow the sharing of information. Levitan (2008) stated,

Vendors and manufacturers are constantly striving to improve the AIMS they offer, with an eye toward promoting records integration. This means not only enhancing communications between systems within one hospital, but also creating a seamless flow of information between unrelated facilities.

Following this response, Noel (1986) responded with more severe criticism. Removing the concerns of development of electronic technology from the discussion, these comments focused on the purpose of the anesthesia record. Not merely a tool for recording data for future use, the anesthesia record was described as, 
...the physician's best tool for conceptually organizing the course of an anesthetic. The act of recording information on the chart forces the anesthesiologist to be aware of the time course and detail of anesthetic events. This awareness is the most important factor in anticipating further events, and correcting untoward events. A mechanically created record, regardless of the facility with which added notation can be made, has the capacity to be formed without ever passing through the consciousness of the anesthesiologist (T. N. Noel, 1986).

Computerized records, rather than a tool to support anesthesia departments involved in medical litigation, could be used as a means to illustrate the inattention of the anesthesia providers assigned to the case. Returning the focus toward the human element, the anesthesia provider, the author indicated that, "Until true machine intelligence is developed, anesthesia recordkeeping should remain a task performed by the human hand" (T. N. Noel, 1986).

Rosen and Rosenweig (1986) rebutted these comments in the next issue of Anesthesiology. Taking exception to the claim that an anesthesia provider required a handwritten record to remain vigilant and aware of the course of events of the case, the authors indicated that a computerized system could be customized to display information that would highlight trends, improving awareness. Other, more advanced uses were also suggested, including the storage of data for future use, as well as the storage and display of policies and procedures, or important but seldom used protocols, such as treatment of malignant hyperthermia (Rosen \& Rosenzweig, 1986). In closing, the authors reiterated 
that it would be the provider, not the recording system, which would ultimately determine the "diagnosis and course of action to be taken (Rosen \& Rosenzweig, 1986).

In this same issue, another letter to the editor offered similar support. Sarnat (1986) indicated that concerns of medical malpractice were only one of many reasons to develop a computerized recordkeeping system. In the author's opinion, the use of the anesthesia record for the purpose of organizing and maintaining awareness of the trends of the patient's course should encourage the development of automation, rather than serve as a hindrance (Sarnat, 1986). The author contended that a handwritten record would not force the attentiveness of the anesthetist, nor would an automated record reduce the provider's awareness of the details of the case. The author likened the development of a computerized recordkeeping system to other technological advancements and their subsequent impact on anesthesia care, such as the electrocardiograph reducing the need for a finger on the patient's pulse (Sarnat, 1986). Sarnat (1986) stated, "Anesthesiologists will not forsake their mission if the clipboard is replaced by an electronic display and they are freed from the tyranny of recording already-acquired data by hand."

\section{Monitoring Standards Developed}

Prior to 1986, monitoring of the physiologic parameters and vital signs of individual patients was largely a decision left to each individual anesthesia provider. Development of standardized guidelines began with the identification of factors associated with critical incidents during anesthesia delivery (Cooper, Newbower, Long, \& McPeek, 1978), as well as the identification of preventable cardiac arrest, a problem 
alleviated in one study by the use of pulse oximetry (Keenan \& Boyan, 1991).

Researchers at Harvard Medical School identified accidents and errors that occurred during the course of anesthesia delivery, along with associated deaths, before and after the implementation of a list of standards of monitoring practice (Eichhorn et al., 1986). Authors found a significant decrease in the number of accidental incidents during anesthesia, as well as a decrease in associated mortality. Based upon these findings, the American Society of Anesthesiologists (ASA) approved similar monitoring standards for all anesthesia delivery in 1986 (ASA House of Delegates, 2005). Following the adoption of these standards of monitoring (Eichhorn, 1993), the frequency and severity of anesthesia related "mishaps," declined significantly (Pierce, 1988) (Eichhorn, 1993). The specialty of anesthesiology would prove to be well advanced in the area of identification of preventable medical errors, well in advance of the hallmark publication by the Institute of Medicine in 1999, "To Err is Human" (Kohn et al., 1999). In response to this landmark publication, the Agency for Healthcare Research and Quality (AHRQ) received $\$ 50$ million from the United States Congress to begin efforts concentrated to identify and prevent medical errors (Report brief. to err is human: Building a safer health system institute of medicine.). The following year, 2001, the Anesthesia Patient Safety Foundation (APSF) initiated a program to advance patient safety through the development of "automated information systems" (Stoelting, 2001).

\section{Advancement of Computing Technology}

By 1990, technology had continued to advance, as had the controversy of the computerized record, spurred by the search for more effective monitoring to adhere to the 
ASA's standards. Hamilton (1990) claimed the computerized record was, "inevitable and valuable." The author spoke of a system that could gather information from standard non-invasive monitors, provide an electronic graphic display, and condense all this information into a succinct and legible printed record. Hamilton defined incentives to the development of a computerized record, accuracy, decreased bias of the recordkeeper, enhanced completeness, improved vigilance by creating additional time to attend to the patient and/or procedure, legibility, and standardization (Hamilton, 1990).

Discussions of implementation and acceptance of computerized systems continued through the year, with Kari (1990) comparing computerized and handwritten charting systems in an ICU. The author found overall acceptance from the nursing staff of the computerized system, and a decrease in errors of recall of hemodynamic trends on the part of physicians (Kari, Ruokonen, \& Takala, 1990). The design and implementation of a computerized medical record system was examined by Gage et al (1990). The importance of the inclusion of the actual users of the system in the design and implementation phase was found to be of paramount importance to ultimate acceptance of the system (Gage, Subramanian, Dydro, \& Poppers, 1990).

\section{Continuing Controversy}

In the midst of these advancements, voices were still raised for caution. Saunders (1990) addressed the controversy by offering contrary views to several of the prominent potential benefits of AIMS. The proposed enhanced accuracy of the computerized record, ultimately resulting in improvements in patient care and safety was questioned, by raising the concern that the anesthesia provider will no longer remain connected to the 
subtle trends of vital signs. The author indicated that the review of previous information (trends) to enhance either prediction of future outcomes, or the result of intervention, has yet to receive adequate study. Saunders (1990) also questioned the belief that a more accurate record would protect providers from "frivolous" malpractice suits. The risk of recoded artifacts erroneously viewed as "instability" of the patient, was cited as a drawback of computerized records (Saunders, 1990). Concerns about the enhanced ability for computerized records to be analyzed for the purposes of quality improvement were raised, by the indication that any queries to the database must be designed by humans, exposing the results to similar degrees of error as with handwritten records. The actual accuracy of monitoring instruments was raised as a contrary point to the enhanced accuracy of computerized monitors, by the removal of human intervention to assess each reading for validity in the context of the anesthetic case. Saunders (1990) indicated that the human practitioners act as a filter to erroneous readings, screening such artifacts prior to recording values, rather than after the fact in terms of explanation of artifacts recorded by AIMS. Manual entry of data that cannot be recorded automatically was also cited as a source for not only potential error or inaccuracy, but also as a time consuming activity that may affect the timeliness of recordkeeping in a crisis (Saunders, 1990). Finally, Saunders (1990) challenged the concept of the improvement of quality of care resulting from computerized recordkeeping as an untested claim.

\section{Advancement Continues}

The first hospital in the United States to adopt an AIMS for full use occurred at Burbank Hospital in Fitchburg, Massachusetts. Beginning in 1988, this facility initiated 
an AIMS project, eventually collecting over 8,000 cases with the loss of only 13 records. Loss of data was “due to user, not equipment, failure." Eichhorn and Edsall (1991) detailed the benefits of enhancements in quality assurance, inventory, and billing as a result of implementation of an AIMS. Cost savings were cited, with the initial cost of $\$ 100,000$ for purchasing and implementation of the AIMS in eight operating rooms, and a savings of $\$ 100,500$ over the first 18 months of use. In response to the concern of lack of timeliness of manual entry, Eichhorn and Edsall (1991) stated, "Eighty-two percent of our manual entries are made within 2 minutes of an event occurring" (Eichhorn \& Edsall, 1991).

Within this same article, Gravenstein (1991) illustrated that the practice of recording heart rate and blood pressure every five minutes originated as a means of time management, as such values were collected by manual measurement, as well as for space limitations on handwritten records. With the removal of these constraints by automated systems, the author advised that much more frequent data collection would result in greater accuracy of trends. The potential rate of change of various physiologic parameters is cited as either "fast" or "slow," with only temperature falling into the "slow" category, indicating a strong argument for more frequent recording of those "fast" parameters (Gravenstein, 1991).

Arguments for use of AIMS in order to produce a record that was not only accurate and timely, but also neatly organized and legible, to enhance credibility in the event of medical malpractice claims were presented by Kroll (1991). The author stated, "The least credible source of fact is a specific memory at the time of deposition. Specific 
memory is no substitute for what is clearly documented in the medical record. In law, the perception of truth is truth. If it is written, it was done" (Kroll, 1991).

Westenskow (1991) suggested the potential development of "artificial intelligence," as a means to not only deal with the recording of artifact, but also to elicit alarms or notifications to the anesthesia provider when predefined limits are exceeded (Westenskow, 1991). Eichhorn (1991) illustrated the possibility that the use of AIMS could extend beyond the operating room, and expand the ability to collect data for quality assurance in any anesthetizing location, even in remote locations. The author also discussed the possibility of encompassing the entire periopertive period, from preoperative interview through postoperative visit, by means of an AIMS (Eichhorn, 1991). Future developments, in addition to the "artificial intelligence" of AIMS, could include the implementation of control systems to assist the anesthesia provider in the selection of agents, the dosage of agents, and the timing of administration, the opportunity to teach high technology to learners in the environment, and even broader connections with other computerized systems both within the hospital facility and between distant facilities (Smith, 1991).

\section{Experience with AIMS / Lessons Learned}

With the implementation of AIMS at various institutions throughout the United States, authors began to share their experiences with systems, offer advice on development and implementation, and espouse both benefits and limitations. Abenstein, et al (1992) discussed eight years of experience with the COMputerized ANesthesia Data Acquisition System (COMANDAS) at the Mayo Medical Center, Rochester, Minnesota. 
After collection of over 24,000 records during this time, benefits cited were enhanced completeness of the record, improved organization of the anesthesia record, and greatly improved legibility. The primary limitation of the system was cited as difficulty with the user interface, particularly the time required to enter data in times of high workload (induction, emergence, invasive line placement, initiation and termination of cardiopulmonary bypass). Additional difficulties included the recording of artifacts, and problems with interfacing with other computers or medical devices. These difficulties led the department to explore the possibility of replacement of the COMANDAS system. Ultimately, no satisfactory replacement could be found, and the facility elected to attempt to update their current system on site (Abenstein et al., 1992).

Zbinden et al (1992) echoed many of the software issues raised by Abenstein et al (1992). Attempts to standardize a software "package" in Switzerland were met with similar obstacles. The goal of this group was to develop a computerized system that integrated administrative and statistical data for the anesthesia department and surgical services, and ultimately to include the intensive care units. The primary hindrance cited was the fact that the introduction of an AIMS “... was not welcomed by the anesthesiologists, nor were benefits perceived for the patient or the anesthesiologist." Despite objection, the system, once implemented, produced significant benefits. Benefits included more efficient billing, increasing income, and improvements in scheduling of cases in the operating rooms, reducing overtime and enhancing cost savings. Difficulties with the user interface, which did not include either "windowing" or use of a computer 
mouse, continued to plaque the system and create dissatisfaction (Zbinden, Christensen, \& Kuster, 1992).

Petry et al (1992) analyzed the use of three different AIMS in cardiac anesthesia. The authors stated, "Automated monitor data record systems are considered to be a prerequisite not only for research in anesthesia, but also for quality assurance" (A. Petry, Gockel, \& Wulf, 1993a). Despite the authors' belief in AIMS, issues of user interface and recording of artifact are cited as sources of significant dissatisfaction (A. Petry et al., 1993a).

The concept of computerization of the preoperative interview was explored, through the use of an automated preoperative patient "interview". A computerized series of questions were presented to 120 patients presenting to the preoperative clinic. Elapsed time was recorded, and found to increase in direct proportion with the age of the patient. Other difficulties identified were patients' feelings of intimidation of computers, preoperative anxiety that resulting in lack of care in responding to questions, and uncertainty as to the importance and relevance of the information provided. The authors stated, "It is important to remember that as good as the computer may be in obtaining historical patient data, it is not a substitute for a complete evaluation by a physician" (Vitkun et al., 1995).

Wang et al (1994) explored the concept of integration of an AIMS with the information management system employed by the hospital. To achieve success, the user interface was designed to be "user friendly" and "fast." In order to achieve this goal, the AIMS was integrated into the hospital's information system. With this connection, a 
great deal of patient information could be accessed by the anesthesia providers and added to the anesthetic record, reducing or eliminating the time required to find and manually enter this data. Data editing was available, to allow anesthesia providers to adjust erroneous data and provide comments, yet the original data was retained to enable auditing of the record at a later date. The addition of "event" keys allowed anesthesia providers to mark the time and occurrence of an event when they were otherwise occupied and unable to fully enter data about the event. These marks acted as place holders, to allow the anesthesia provider to return to the exact time and expand on the nature of the event once workload was reduced. Time and motion studies were preformed on 44 cases prior to the implementation of the system, and on 41 cases after the AIMS were employed. Results indicated a reduction in the time spent on recordkeeping activities, from $20.4 \%$ of the case with handwritten records to $13.4 \%$ with use of an AIMS. There was also an significant increase in the time spent in "arranging equipment," from $6.4 \%$ to $8.1 \%$, time spent in "patient preparation," from $10.1 \%$ to $13.1 \%$, and time spent in "non-anesthesia activities" from $6.3 \%$ to $11.3 \%$ of the total time spent during the anesthetic case (Wang, Gardner, \& Seager, 1995). The classification of "non-anesthesia activities," was not defined by the authors.

Perhaps in contrast to these results, Henrichs (1994) called to attention the discussion of some disadvantages of a computerized system. The author stated, By going through the process of entering data on the course of the anesthetic procedure on the protocol sheet, the information is mentally absorbed and evaluated by the anaesthetist. This information may, 
however, be lost when the data are recorded fully automatically - without active involvement on the part of the anaesthetist. Studies on human performance are needed to elucidate the effect of automated records on anaesthesia quality (Heinrichs, 1995).

The author indicated that such a fully automatic system was not in place at the Clinic for Anesthesiology at Johannes Gutenberg University, Mainz, FRG, Medical School, Germany, for these very reasons. The perception of the anesthesiologists at the time was that, "We feel that fully automated anesthesia records could be used only if intelligent alarms are integrated into these systems" (Heinrichs, 1995).

\section{Vigilance}

By the year 1989, computing technology had advanced to a point where a fully automatic recording of a patient's physiologic parameters and vital signs was widely available. Various systems were beginning to become implemented in both academic centers as well as private practice facilities both in the United States and abroad. In many of these facilities, obstacles to complete acceptance and satisfaction continued to surface in the form of concerns about the effect of computerized charting on the overall vigilance of the anesthesia provider.

Vigilance as affected by the workload of the anesthesia provider has been studied long prior to the advent of the AIMS of the 1990's. A definition is offered for vigilance as a task, "which requires the detection of changes in a stimulus during long monitoring periods when the subject has little or no prior knowledge of the sequence of the changes"(Olmedo \& Kirk, 1977). In 1982, Paget et al examined the tasks of the 
anesthesia provider and the effect of these tasks on the concept of vigilance. The authors cited the effects of "time-sharing," "the form of the stimulus," "signal frequency and strength," "environment," "noise," "gas pollution," sleep deprivation," and "end-spurt," on the vigilance of the anesthesia provider. "Time-sharing" referred to the fact that anesthesia providers must conduct many tasks of equal importance simultaneously. The authors stated, "Only rarely is performance enhanced; whereas increasing the time and complexity of the job are most likely to result in performance decrements, if the level of arousal of the individual to the tasks can be maintained then there may be no decrement" (N. S. Paget, Lambert, \& Sridhar, 1981a). “The form of the stimulus," was identified as a significant factor to vigilance, with more complex stimuli (complex displays, integration of audio and visual displays and alarms) resulting in enhanced vigilance rather than reduced vigilance, despite divided attention. "Signal frequency and strength," indicated that the rate of stimuli presentation produced conflicting results, with some subjects responding more accurately to fast paced signals, yet when fast paced signals were combined with a reduced strength of signal (lower volume or dim display), accuracy of response degraded. "Environment," referred to the state of awareness of the subject. In the case of the anesthesia provider, factors such as noise level or exposure to waste gases could reduce alertness, resulting in a diminishment of vigilance. "Noise," a selfexplanatory term, referenced the effects of several types of noise (quiet, speech, music, industrial noise) presented either in a continuous or intermittent manner. Of these, the continuous presence of music resulted in the least effect on the subject's vigilance, although the authors indicated that further study was indicated. "Gas pollution," referred 
to the presence of waste anesthetic gases in the environment, a factor virtually eliminated by the use of scavenging systems for waste gases in modern operating rooms. "Sleep deprivation," clearly indicated a progressive loss of vigilance with increasing periods of lack of sleep (N. S. Paget, Lambert, \& Sridhar, 1981b). Performance returned to previous levels following a period of rest. Finally, the concept of "end-spurt," improved performance as the overall task approached its endpoint, was unable to be fully explained, with the authors suggesting further study (N. S. Paget et al., 1981b). Many of the issues raised by the work of Paget et al (1981) would become topics of study for many years to come (Weinger \& Englund, 1990).

The examination of the use of automated systems, specifically that of automated non-invasive blood pressure readings, received attention in 1986. Kay and Neal (1986) studied vigilance through the occlusion of the ear piece of a group of residents taking manual blood pressure readings, and a similar group of residents using an automated blood pressure device (ABPD). Results noted a significant decrease in the use of earpiece listening devices in the group using an ABPD (24\%) as compared to the group taking manual blood pressure readings (65\%). Total time (in seconds) before recognition of loss of auditory data from the earpiece was significantly lower in the group utilizing the automated system (108 \pm 66 seconds), as compared to $58 \pm 61$ seconds in the manual blood pressure group. The authors cited an arbitrary 180 second "cut-off" time for discovery of the clamping of the earpiece as a source of potential underestimation of results in the automated blood pressure device group. The authors stated, "Although ABPDs have been hailed as a major advance in monitoring, our study suggests that 
slower response times to monitoring interruption occur in a training program that relies exclusively on such devices” (Kay \& Neal, 1986).

In an abstract, Yablok (1990) compares vigilance of anesthesia providers using an AIMS and those using handwritten records. Twelve providers, both anesthesiologists and CRNAs were studied during a total of 5 cases using each of the two recordkeeping modalities. The researcher approached practitioners during the maintenance phase of the anesthetic. Practitioners were asked to turn away from the patient and monitors and recall the values of seven physiologic parameters; blood pressure, heart rate (HR), endtidal carbon dioxide level $\left(\mathrm{ETCO}_{2}\right)$, inspired oxygen concentration $\left(\mathrm{FiO}_{2}\right)$, oxygen saturation $\left(\mathrm{SpO}_{2}\right)$, peak inspiratory pressure ( $\mathrm{PiP}$, and temperature. Any deviation from values currently displayed on the monitors was counted as error. Results indicated that "in over one fifth of the AR \{automated record\} cases, the providers could not give the value of one or more variables" (Yablok, 1990), p. A416).

Also in 1990, Weinger and Englund replicated much of the work of Paget et al (1981). Listing factors that affect vigilance and performance of anesthesia providers, the authors also discuss the possibility of automation as a potential solution, but suggested caution. The authors stated, "There are several reasons to automate complex systems: to enhance system performance, to increase safety, and to reduce human workload. However, automation will not necessarily lead to improved system performance in every situation" (Weinger \& Englund, 1990). Weinger and Englund (1990) called for "intelligent" systems, that could incorporate alerts and alarms that could trigger responses from the anesthesia provider, rather than add to the volume of monitoring devices that 
require attention; attention diverted from direct patient observation and care. The authors asked, "Does a new device provide sufficient additional information (or early warning of some critical condition) to justify its cost, both in terms of financial economy and in terms of decreased use of the already available monitoring strategies?"

The aspect of time spent keeping an anesthetic record was added to a study of vigilance that compared use of an AIMS with MER in 1995. Allard et al (1995) studied 33 residents and 8 CRNAs over the course of 76 anesthetic cases. Subjects were videotaped to determine the amount of time spent in various categories of activities, and were asked by a researcher to turn away from the patient and monitors and recall values for physiologic data - systolic and diastolic blood pressure, $\mathrm{HR}, \mathrm{PiP}, \mathrm{ETCO}_{2}, \mathrm{SpO}_{2}, \mathrm{FiO}_{2}$, and temperature. Results indicated that there was no significant difference in the time spent in recordkeeping tasks, and that recordkeeping accounted for $10-15 \%$ of the total time of each anesthetic case. Results of the vigilance study revealed a low percentage of incorrectly recalled values (11.56\%) for AIMS group as compared to the MER group $(8.11 \%)$, results that were not statistically significant. Fewer errors were noted in the AIMS group for the values of systolic blood pressure and oxygen saturation, indicating to the authors that these areas could have held a higher degree of significance to the subjects than other physiologic values (Allard et al., 1995).

Loeb (1995) studied nine anesthesiology residents over a series of 18 cases to determine the presence of differences in the performance of a "vigilance task" when asked to keep a handwritten record, or when the handwritten record was kept by a "scribe," an anesthesiologist not otherwise involved in patient care (Loeb, 1995). This 
work expanded on the author's previous work, which sought to define the degree of vigilance during three phases of anesthesia delivery - induction, maintenance, and emergence (Loeb, 1994). Loeb (1995) sought to examine the response rate of anesthesia residents to a change of a numerical value displayed alongside other physiologic parameters for the patient. The presence of a human "scribe," was intended to serve as a surrogate for an electronic recordkeeping system. The author stated, "This was not a study of the effect of current electronic record keepers on vigilance. Rather, we investigated whether the clinician must perform the record-keeping in order to maintain vigilance." Results of the study indicated that there were no significant differences between the groups in frequency of response or in time to response based upon the two groups. The author cited the use of the experimental numerical value as a possible limitation, in that the number had no value to actual patient condition, and further indicated that the use of recall of vital signs in previous studies was more an assessment of memory rather than vigilance (Loeb, 1995). No mention of the possibility of an expectancy (Hawthorne) effect to the vigilance task was discussed.

This work by Loeb (1995) was questioned in an article by Woods et al (1995). The authors indicated that the work by Loeb (1995) did not fully assess the impact of AIMS, as the presence of a human scribe would serve to provide an additional source of interaction, or another "team player". Woods et al (1995) indicated that "automated systems often fail as team players." The authors described AIMS as,

Strong, when they can act autonomously; silent, when they can provide poor feedback about their activities and intentions; clumsy, when they 
interrupt their human partners during high workload and high-criticality periods, or add new mental burdens during these high-tempo periods; difficult to direct, when it is costly for the human supervisor to instruct the automation about how to change as circumstances change. Systems with these characteristics create new problems for their human partners and new forms of system failure (Woods, Cook, \& Billings, 1995).

The authors define vigilance in terms of varieties of attention, in particular as "sustained attention, stating, "Sustained attention is what cognitive psychologists call vigilance.". Attention is further divided into "divided attention and attention switching." Divided attention is used by providers during periods of "overload", and includes, "time sharing, shedding loads, task priorities, and shifting workload over time" and is used during periods of high activity, as induction and emergence. Attention switching is used during periods of "underload," or during the maintenance phase in order to predict or detect the onset of a problem early in development. Based upon these definitions of vigilance, the authors indicated that Loeb's (1995) "vigilance task" was ineffective, as it was either too easy to complete, did not compete for similar resources (attention), or that the primary task required a low allocation of resources (attention). The authors concluded, "Studies of the impact of automation on human performance show that following this logic \{if a scribe is useful, automation will also be beneficial\} frequently produces automated devices that create new cognitive burdens, especially during high-tempo periods and nonroutine cases" (Woods et al., 1995). 
The role of AIMS was studied by Weinger et al (1997) in cases with a significant degree of "high-tempo periods," particularly anesthetics for patients undergoing cardiac surgery, with the inclusion of an additional task, transesophageal echocardiography. In this study, recordkeeping method was randomly assigned to the practitioners prior to each case. Twenty cases were studied, evenly divided between the two recordkeeping groups. Activities of the nine anesthesiology residents were monitored by a trained observer, and divided into 32 categories. Response to a "vigilance task," an "alarm light" mounted beside the electrocardiograph (ECG) monitor was recorded in seconds elapsed from the time of illumination until detection by the provider. Results indicated,

When all cases were included, the greatest amount of time was spent after intubation observing the monitors $(24.7 \pm 1.5 \%)$, recordkeeping $(11.5 \pm$ $0.6 \%)$, adjusting the intravenous tubes $(8.1 \pm 0.8 \%)$, and adjusting or observing the TEE $(7.7 \pm 1.1 \%)$. Both groups showed similar mean dwell times on nearly all task categories, including recording (Weinger, Herndon, \& Gaba, 1997).

The authors also indicated that both groups were similar in the amount of time spent in both direct and indirect patient care tasks, or in response time to the vigilance task.

Overall, no differences were discovered between the AIMS and MERS group on tasks of workload or vigilance (Weinger et al., 1997).

\section{Accuracy}

A second aspect of the controversy between the adoption of AIMS over existing MER systems is that of accuracy. With the advent of an alternative method to 
handwritten recordkeeping during the delivery of an anesthetic, critics called into question the benefits of computerization of the process. Prior to the advent of monitoring standards in 1986, the composition and organization of handwritten records varied by institution and even practitioner. The quality of such records often suffered from poor handwriting, rendering even complete records illegible to all but the practitioner responsible for the chart. Zollinger et al (1977) compared the composition of 100 records handwritten by anesthesia residents to those generated simultaneously by a computerized system. Residents recorded vital signs every five minutes, and the computerized system recorded vital signs in one minute intervals. Results revealed, "major discrepancies between the computerized and handmade records in 43 per cent of the patients". Residents recorded blood pressure readings $94 \%$ of the time, with the computerized system recording blood pressure $78 \%$ of the time; heart rate entries were noted 54.7 times per hour with computerized records, and only 10.8 times pefr hour on handwritten records. The authors noted that, "Significant changes in data were often underestimated by humans who tended to smooth out a record. In addition, a computerized monitoring recording tends to pick up changes when the anesthesiologist is otherwise occupied" (Zollinger et al., 1977). Such discrepancies would be noted over the course of many comparisons between computerized and handwritten records in years to follow, as would voices of caution that computerized charting should not replace direct observation of the patient.

The next study of accuracy of a computerized method of generating an anesthesia record would not appear in the literature for ten years. Logas et al (1987) offered an 
unpublished abstract detailing the comparison of $\mathrm{HR}$, and systolic and diastolic blood pressures as recorded on a handwritten record and collected by a non-invasive blood pressure monitor that also collected HR. Results revealed "significant discrepancies" between the two methods, particularly during induction and emergence from anesthesia (Logas, McCarthy, Narbone, \& Ivankovich, 1987). Discrepancies of more than 20 points were noted in $84 \%$ of the 101 cases measured, with an average of 2.3 occurrences per case.

A year later, Lerou et al (1988) studied differences between handwritten and automated values for eight physiologic parameters: tidal volume (TV), breathing rate, $\mathrm{ETCO}_{2}$, oxygen fraction in the anesthetic circuit $\left(\mathrm{FiO}_{2}\right)$, oxygen saturation $\left(\mathrm{SpO}_{2}\right)$, systolic and diastolic blood pressure (SBP, DBP), and HR. Thirty patients presenting for elective eye surgery were anesthetized by one of 3 anesthesiologists or six senior anesthesiology residents. Both a handwritten record and an automated record was generated for each patient, with a detailed evaluation of differences between the two records. The authors found that, "during the study our group of anesthesiologists updated their records with blood pressure and HR data at a mean interval of six minutes. Other variables were less frequently updated" (Lerou, Dirksen, van Daele, Nijhuis, \& Crul, 1988). Many instances of missing values were noted in the handwritten records, particularly for the periods of induction and emergence, when anesthesiologists entered data onto the handwritten record from memory. The authors calculated an "error fraction," for erroneous or missing data recorded by each method. For the handwritten records, an error fraction of 0.23 to 0.31 was calculated for $\mathrm{TV}$, breathing rate, $\mathrm{ETCO}_{2}$, 
$\mathrm{FiO}_{2}$, and $\mathrm{SpO}_{2}$. The error fraction for these same variables was calculated as $0.01-0.06$ for the computerized recordkeeping system, indicating a greater degree of accuracy. The authors noted, however, instances of erroneous data for the automated record, a widely held concern of computerized systems. Such artifacts as electrocautery interference with electrocardiograph (ECG) readings were minimized by an "averaging" feature of the automated recordkeeping system employed by the authors for this research. Despite the presence of artifact, the authors concluded, "The infrequent hand charting of important physiological variable indicates that even eight physiological variables are too many to record manually with a high update frequency. Therefore, a manually kept record may not be as useful in helping detect trends as is an automated record" (Lerou et al., 1988). In a study of 46 patients anesthetized by 32 anesthesia providers ( 20 anesthesiologists and 12 CRNAs), handwritten records were generated by the provider and compared to blood pressure recordings generated by an automated blood pressure monitor to determine accuracy of handwritten anesthesia recordkeeping. Cook et al (1989) noted that not a single record indicated a diastolic blood pressure higher than 110 $\mathrm{mmHg}$, while diastolic blood pressures greater than $110 \mathrm{mmHg}$ occurred 33 times in 15 cases as recorded by the automated blood pressure monitor. Systolic blood pressure readings of $170 \mathrm{mmHg}$ or greater were recorded by the automatic device in 11 cases, yet only in four of the handwritten records. The authors stated,

It is unlikely that the extreme readings absent from handwritten records were simply missed by the record keepers. Although a single reading might well be missed (for example, during intubation) most automatic 
records contained multiple instances of readings above the highest handwritten entry. Manual record keepers had, on average, more than three opportunities to capture and record a systolic pressure greater than the highest one they did record (Cook, McDonald, \& Nunziata, 1989).

The authors did not attempt to claim that automated recording of vital signs was superior to handwritten records, rather they relied on their data to present that there was a definitive difference between the two methods. Further, the authors did not attempt to explain the cause for such discrepancies, offering only, as did Zollinger et al (1977), that "physicians like smooth charts inasmuch as they imply a better management of the case" (Cook et al., 1989)). These findings of smoothing were further documented by researchers in 1990 (Shibutani, Bairamian, Subhedar, Kashiwagi, \& Kubal, 1990), and 1991 (F. E. Block Jr., 1991).

The controversy of automatically recorded artifact data was addressed by Gage (1992). The author offered a solution to erroneous readings through the programming of redundant signal channels used to record identical data. Just as a clinician observes trends, an automated system could be programmed to take multiple readings simultaneously, through separate channels, to reach an "average" value that would be more accurate than that recorded by only a single channel (Gage, 1992). This concept is similar to the "averaging" programming used by Lerou et al (1988).

An oft cited study of comparison between AIMS and MERS was conducted by Thrush in 1992. In this research, thirteen handwritten anesthesia records were compared with 13 records simultaneously generated by an AIMS. The handwritten records were 
recorded by anesthesiology residents, with the computerized records generated by an AIMS operated by an anesthesiologist not otherwise engaged in patient care. The author arbitrarily defined acceptable ranges of values for 5 physiologic parameters: SBP, DBP, $\mathrm{ETCO}_{2}, \mathrm{HR}$, and $\mathrm{SpO}_{2}$. Deviance from the acceptable range of values was noted for each record, and instances of deviance compared between the two methods of recordkeeping. Statistically significant differences were revealed for low values of SBP and DBP, high values of $\mathrm{ETCO}_{2}$, and low values for $\mathrm{HR}$; in each case, the automated system recorded more instances of the out-of-range values than handwritten records. Methodological problems, including a small sample size of thirteen patients, and failure to control the similarity of subjects and cases, plagued the research. Selection of anesthetic cases resulted in widely dissimilar procedures. Of the thirteen cases selected, eleven consisted of patients for cardiac surgery requiring cardiopulmonary bypass (CPB). One case was that of a patient receiving a cranioplasty procedure, and the thirteenth case was that of a patient for excision of a wrist ganglion. Inclusion criteria stated by the author was, "Adult patients scheduled for operations that required general anesthesia for longer than 1.5 hours." Of the thirteen cases included in the study, only nine cases were studied for the full 1.5 hours, four cases required cessation of observation due to the need for "early initiation of CPB." Subjects in the study produced disparate numbers of records; as stated, "One resident created three records, three residents created two records each, and four residents created one record each.” Despite the presence of these methodological flaws, the authors contended that results "support the use of automated, rather than manual, anesthesia records" (Thrush, 1992). 
The concerns of time spent in recordkeeping tasks, and overall quality of the anesthetic record, were explored by the multidisciplinary team of Edsall et al (1993). Ten patients presenting for arthroscopic surgery of the knee were anesthetized by three CRNA volunteers; one CRNA participating in six cases, with the remaining CRNAs participating in two cases each. Each CRNA completed an equal number of anesthesia records with AIMS and MERS. Each case was videotaped in its entirety, and reviewed by a researcher who timed the time of the anesthetic and the total amount of time spent on documentation with a stopwatch. Quality of the anesthetic record was determined by the amount of illegible or incorrect entries by either method of recordkeeping. Completeness of the anesthetic record was determined by the inclusion of 46 predetermined elements of patient care, including demographic data about the patient, appropriate frequency of notation of vital signs, and narrative data about procedures and events of the anesthetic. Average length of case was similar for the two groups, with MERS cases lasting an average of 31.78 minutes, and AIMS cases lasting an average of 33.76 minutes. Results of the study indicated that significantly more time was spent in recordkeeping activities with handwritten records (11.9 minutes per case) than with the AIMS (4.95 minutes per case). Frequency of artifact was not significantly different between the two groups. AIMS records were found to be more complete in both clinical items and vital signs. A total of 31 clinical items were absent in the AIMS group, as compared to 51 missing items in the MERS group. The AIMS group did not miss any vital sign data, while the MERS group had 71 absences of data. Methodological concerns of this study include the extremely small sample of three subjects generating a total of 10 
cases, equally divided between AIMS and MERS recordkeeping methods. Other concerns involve the fact that handwritten records had not been used in this facility, or by two of the participants in the previous 2.5 years, although all three CRNAs had extensive experience with the AIMS utilized in this study, logging over 300 cases each over a prior six month period. Despite these limitations, the authors submitted that, "In addition to producing a more complete, accurate, and easily reviewable record for quality assurance and other review purposes, AIMS's require proportionately less time than manual systems for anesthesia record keeping, thereby allowing more time for patient observation and direct patient care"(Edsall et al., 1993).

\section{Completeness}

In accompaniment to the work of Edsall et al (1993), critical elements for inclusion in the anesthetic record were the subject of a study by Biddle et al (2001). Based upon the standards of monitoring and record keeping of the ASA and AANA, thirteen variables were identified as important for inclusion in an anesthetic record. These variables included: patient identification, surgery and/or anesthesia start time, anesthesia provider name(s), heart rate/rhythm, blood pressure, oxyhemoglobin saturation, end-tidal carbon dioxide, $\mathrm{FiO}_{2}$, or $\mathrm{O}_{2} / \mathrm{N}_{2} \mathrm{O}$ /air liter flow, volatile agent concentration, airway management, intravenous fluid, emergence from anesthesia, and surgical positioning (Biddle et al., 2001). Through a multicenter study that included 4 academic medical centers and 5 community hospitals, a total of 4,989 anesthesia records were tabulated for missing variables. Results indicated that the variable, "emergence from anesthesia," was absent in $28.6 \%$ of records. "Surgical positioning" was absent in 
$18.9 \%$ of records, with "surgery and/or anesthesia start time," absent in $13.1 \%$ of records. All other variables were absent in less than $10 \%$ of records, with "patient identification" present on every chart evaluated.

The authors suggested that the high volume of tasks associated with emergence of anesthesia, coupled with performance pressure for efficient operating room turnover, could have resulted in the unusually high rate of absence of the variable "emergence from anesthesia" (Biddle et al., 2001). These findings are consistent with the findings of Edsall (1993), and serve to support the findings that periods of high workload may have a significant effect on recordkeeping.

\section{Financial Incentives}

Many proponents of electronic recordkeeping have cited improved capture of billing elements associated with anesthesia delivery as a distinctive benefit (Edsall et al., 1993) (Lubarsky et al., 1997). In 1997, Lubarsky et al examined use of the AIMS to perform and implement cost containment practices within the anesthesia department at the Duke University Medical Center in Durham, NC. This work was an expansion of previous research by the authors who cited in an abstract a cost savings of approximately one million dollars per year by introducing practice guidelines for pharmaceutical use (Lubarsky et al., 1996). In this research, total costs of muscle relaxants, induction agents, anxiolytics, opioid analgesics, fluids, and volatile anesthetics, were studied to determine usage practices and total costs, with 99\% accuracy (Lubarsky et al., 1997). Use of various muscle relaxants were examined by length of surgical case, average fresh gas flows were measured to determine total usage of volatile anesthetics, and individual pharmaceutical 
costs per provider were calculated. Results were considered by five "best practice committees," consisting of four to six members per committee, who examined the results of the study and created suggestions for cost containment. As a result of this research, practice guidelines were implemented at the facility to reduce pharmaceutical costs. In particular, individual pharmaceutical costs were calculated, and this information shared with each provider, and a reward program was instituted to provide practitioners with financial incentive in the form of an educational expense account for practitioners with high degrees of compliance. In this way, the authors felt certain to enhance or at least maintain cost savings through practice guidelines created through the use of the extensive database complied by the AIMS (Lubarsky et al., 1997).

A year later, in 1998, an estimate was published that only $1 \%$ of all anesthesia departments within the United States were presently utilizing AIMS (Thys, 1998). Thys (1998) cited enhanced capture of billing elements as one of several benefits that substantiate the claim that the use of AIMS was "essential” (Thys, 1998). Enhanced billing services included more accurate documentation of services provided to the patient, more accurate accounting of costs with the ability to produce cost-containment through practice guidelines, and improved utilization of resources to improve efficiency and limit waste of time and materials.

Reich (2006) explored the possibilities of improved efficiency through use of an AIMS, developing a program to obtain billing elements directly from the computerized anesthesia record at the time of service, rather than at a later time by billing personnel using paper vouchers. The program examined every record generated by the anesthesia 
department on a daily basis, and identified any missing elements that would be required for payment of services rendered. Billing elements included electronic signatures for all anesthesia personnel, patient demographic information and diagnosis, anesthetic technique and placement of invasive monitors, and surgical procedure preformed. Use of this program reduced "charge lag" (time elapsed from date of service to submission of charges to the payer) by 7.3 days, and direct collection of billing elements eliminated the need for a full-time employee assigned to anesthesia charge reconciliation, a cost savings determined to be $\$ 32,000$ per year. Further cost savings were realized by decreased costs for printing, transporting, and filing of records, a reduction of an additional $\$ 10,000$ per year. The authors cited the cost savings, enhanced accuracy of billing elements, and reduction of "charge lag," as justification for the purchase, implementation, and maintenance of an AIMS (Reich et al., 2006)). In an accompanying editorial, concerns of "intellectual property," the question was raised, "If the hospital owns the AIMS, who owns the data generated from the AIMS?” (Abouleish \& Conlay, 2006). The author cautions readers to carefully consider this question prior to development and implementation of such a system.

The concept of accuracy of documentation of all elements necessary for acceptance and reimbursement from third-party payers was further explored in 2007. The authors developed software that would examine each anesthetic record for completeness, and automatically notify practitioners by e-mail of missing elements in need of correction. Over the course of this research, cases that could not be billed were reduced from $3.4 \%$ of all cases to $0.04 \%$ of all cases. Time elapsed from identification to 
correction of these billing elements was reduced from an average of 33 days to 3 days. Time to release an anesthetic bill to the payers was reduced from an average of 3.1 days to an average of 1.1 days. Overall, more than $90 \%$ of anesthesia providers found the system easy to use. The authors stated that calculations of cost savings indicated, “...the time to return on investment was one half year" (Spring et al., 2007).

This "return on investment" concept was further refined by O'Sullivan et al (2007). The authors identified four areas of benefits unique to AIMS recordkeeping that enhanced billing and reimbursement, justifying the initial cost of investment in the system. The first area of benefit, the reduction of pharmaceutical costs, could be realized by the development, implementation, and adherence to practice guidelines for anesthetic agents. In addition to a reduction in costs, the authors suggested that use of pre-labeled, bar-coded syringes could also enhance patient safety by the reduction or elimination of medication errors. The next area of benefit provided by AIMS is that of a reduction in anesthesia staff costs through enhanced efficiency of scheduling of staff and cases. Through the use of an AIMS, the efficiency of each provider and utilization of each operating room or surgical service could be examined and improved. Such an analysis could have the effect of more efficient scheduling of cases in the operating theater, a more efficient "flow" of cases each day, resulting in the reduction or more efficient utilization of anesthesia and operating room staff. Enhanced capture of billing elements and charges was cited as the next benefit to the implementation of an AIMS. The reduction of "charge lag" and more accurate accounting of materials are identified as benefits that should easily gain acceptance when offered as justification for the initial 
cost of the system. The final benefit identified by O'Sullivan et al (2007) was that of enhanced billing for the hospital facility through more accurate documentation of diagnosis and procedure charge coding. All four benefits are well supported by the relevant literature, with an authors' note that, "Since our paper was accepted in April 2005, 5 additional papers satisfying the study criteria were published" (O'Sullivan, Dexter, Lubarsky, \& Vigoda, 2007).

Findings from O’Sullivan et al (2007) were cited by Egger Halbeis and Epstein (2008) as arguments to present to hospital facilities reluctant to provide initial financial outlay for purchase and implementation of an AIMS, despite similar utilization by other services within the facility (radiology, or pharmacy) (Egger Halbeis \& Epstein, 2008). Potential impediments to purchase of an AIMS offered by hospital administrators were cited as lack of understanding of benefit to the institution, despite clear evidence of benefit to the anesthesia department. The fact that in many facilities, anesthesia services are provided by a private group contracted for service to the hospital is indicated as a cause for such beliefs by the institution. Illustration of these four benefits, particularly that of enhanced accuracy of billing elements for the hospital, were cited by Egger Halbeis and Epstein (2008) as part of a "value proposition" to administrators who failed to recognize many of the potential benefits of an AIMS over more traditional handwritten anesthesia records (Egger Halbeis \& Epstein, 2008).

\section{Enhanced Medico-legal Protection}

The concept of enhanced medico-legal protection of the practitioner by use of automated anesthesia recordkeeping systems has been likened to the use of cockpit data 
recorders in the airline industry (Gibbs, 1989b). Comparisons between the delivery of anesthesia and piloting a commercial airliner have long been illustrated. Similarities between the two industries have been linked through safety checklists (pre-flight versus anesthesia machine checkout), the intensity of workload at the beginning and end of the case or flight (take-off and landing versus induction and emergence from anesthesia), and the paucity of stimulation during the majority of an uneventful case yet still requiring intense vigilance to prepare for swift response to any unexpected event (Gibbs, 1989b). The benefit of AIMS to serve as a flight data recorder for an anesthetic was illustrated by Gibbs (1989), and almost immediately refuted. In an editorial to the Journal of Clinical Monitoring, Gaba (1990), an author known for work with human error and patient safety, countered the arguments presented by Gibbs (1989). Gaba (1990) suggested that AIMS were limited by the accuracy of man-made and frequently non-invasive devices monitoring a human patient, while flight data recorders directly documented the functions of mechanical and electronic systems of a mechanical airframe. The potential for erroneous data recording by AIMS of the human patient would limit the accuracy of any documentation produced (Gaba, 1990). The concern of artifact has long been a common theme among practitioners considering the risks and benefits of AIMS. Concerning the medico-legal aspects of AIMS, Gaba (1990) stated, "Paradoxically, were it not for the medico-legal environment, the profession \{anesthesiology\} might have more quickly adopted many aspects of automated recordkeeping, with all its current limitations and flaws, because it would have benefits with few risks" (Gaba, 1990). 
An illustration of actual data generated and unrecorded by an AIMS was made in 1994. Through three case studies, Feingold et al (1994) cited episodes of severe bradycardia in the form of sinus pauses of up to 5 seconds requiring treatment with atropine for correction that were not recorded by the AIMS in use at the time. These omissions were noted to be due to the fact that data was recorded by the AIMS at a maximum frequency of 30 seconds (Feingold, Galindo, \& Feingold, 1994). In one case, placement of a permanent cardiac pacemaker was required due to recurrences of significant and symptomatic bradycardia (Feingold et al., 1994). The authors cited the omissions noted in these three cases as examples of significant risk of automated recordkeeping systems and the need for sustained vigilance on the part of the anesthesia provider assigned to the case (Feingold et al., 1994).

Continued concerns of accuracy and potential exposure to medical malpractice claims prompted a survey of anesthesia departments utilizing AIMS in 2004. Of particular concern were two scenarios, 1) documentation of actual data that is both innocuous and self-limiting, and 2) documentation of erroneous data or artifact by the AIMS. In each scenario, the author contended that most practitioners would choose not to record this data, relying on professional judgment that such data was of little benefit to the case (Feldman, 2004). Such data, as recorded by an AIMS, could serve as potentially damaging, albeit erroneous, data in the event of a poor outcome for the patient and subsequent medico-legal proceedings (Feldman, 2004; Feldman, 2004) The opposing viewpoint illustrates that anesthesia records generated by an AIMS would be more timely, more complete, and more legible than the equivalent report generated on a 
handwritten record. To research the validity of each point, the author developed and distributed a survey to 55 anesthesia departments utilizing AIMS for anesthesia recordkeeping. Of these 55 surveys, 22 were returned sufficiently complete for inclusion for comparison). Results of the survey indicated that in no case was the anesthetic record generated by the AIMS determined to serve as a hindrance to the defense of practitioners. Comments attached to the survey results indicated that in some cases, practitioners using AIMS felt that the system served to prevent malpractice claims. The practitioners felt that AIMS could reveal unsafe practices prior to a critical incident that could result in a lawsuit (Feldman, 2004)). Other comments also indicated that not only did the objectivity and legibility of an AIMS record serve to benefit the defense, in some cases the record implicated the practitioner's obvious lack of vigilance, resulting in a settlement with the plaintiff (Feldman, 2004). As a tool for defense of anesthesia practitioners, results of this survey indicated that such a record was most accurately described as objective; exonerating the innocent and implicating the guilty.

Despite the benefits of AIMS records in medical malpractice lawsuits, concerns of artifact and missing data prevailed. In 2006, Vigoda and Lubarsky document a case report in which a significant failure of the AIMS to capture patient data resulted in the implication of the anesthesia provider managing the case in a medical malpractice suit. Over the course of a craniotomy for a patient with a brain tumor, some 90 minutes of data was not captured by the AIMS. This AIMS contained software that allowed multiple "windows" of data could be displayed. Each window occupied the entire display screen, yet only the "home" screen displayed incoming data from the patient monitors. The loss 
of data capture was unrecognized by the anesthesia provider, who had a "summary" window displayed, and was unable to view incoming data.. Upon recognition of this issue as a result of a change of anesthesia providers, the problem was rapidly corrected, but the data was not entered manually. Upon emergence, the patient was returned to spontaneous respirations, but there was complete loss of motor function of the extremities. Over the course of the lawsuit, the anesthesia provider assigned to the case was charged with negligence due to the lack of proper monitoring of the patient, and the recording of data at five-minute intervals as required by the monitoring standards of the ASA (Vigoda \& Lubarsky, 2006a). Loss of data was determined to be due to the simple disconnection of a single cable between the patient monitors and the AIMS. Also unique to this lawsuit was the evidence of documentation by the attending anesthesiologist of physical presence on emergence; this documentation was determined to have been entered within minutes of the beginning of the case, rather than at the end of the case, as would be appropriate. By entering this data immediately after induction of anesthesia, the anesthesiologist cast doubt as to whether or not he/she was actually physically present on emergence from anesthesia. While there was evidence that indicated that the anesthesia provider displayed vigilance in patient care and was aware of vital signs that were unrecorded, the lack of documentation of vital signs and evidence of inappropriately timed documentation of other elements were "difficult to defend". The outcome of this case resulted in significant changes in documentation practices at the facility as well as a modification of the AIMS software by the manufacturer to add a "pop-up" window that would indicate the loss of incoming data (Vigoda \& Lubarsky, 2006a). 
Timeliness of documentation was examined by Vigoda and Lubarsky in an article published just one month later in 2006. The difficulty in the maintenance of the timeliness of required documentation when an anesthesiologist is supervising the anesthesia delivery of more than one operating room was illustrated, and differences in electronic and manual records were examined. The identification of an "audit trail," or the electronic record of the time of entry of documentation in an AIMS record, by medical malpractice lawyers has resulted in a significant reduction of "prospective charting" - documentation of events that have yet to occur - by anesthesia providers. Due to the lack of accurate determination of the timing of entries made on a handwritten anesthetic record, such prospective charting was, "according to private communications, not uncommon in many practices." Through educational sessions, e-mail contact of performance/compliance, and subsequent e-mail "copy notification" to the department chairman, documentation timing accuracy improved to $99.5 \%$ at the authors' facility. Through such actions, prospective charting - a practice potentially damaging to the integrity of the anesthesia care team - was virtually eliminated (Vigoda \& Lubarsky, 2006b).

\section{Quality Improvement}

With the development of electronic medical records, the potential advantages of computerized database systems over traditional handwritten records were quickly recognized. Directed review of a medical record by query entries showed the potential to improve the speed and quality of analysis of a patient's entire medical history (Whiting-

O'Keefe, Simborg, Epstein, \& Warger, 1985). Automation of an anesthetic record could 
also carry the added benefit of increased data through shorter sampling intervals, rather than the standard 5 minute documentation of vital signs. In addition to reducing the workload of the anesthesia provider by eliminating the need to manually record each value, an automated system could be programmed to record values much more frequently than could be feasibly recorded on a handwritten record. For some rapidly changing vital signs, such enhancement could result in a more accurate record of events of anesthesia and surgery (Gravenstein, de Vries, \& Beneken, 1989).

Once recorded, the anesthesia record could be consolidated into a large database, where each record produced could be stored electronically for future retrieval. Review of this database could potentially result in recognition of trends of poor or inefficient performance, or even the identification of practices that could prove detrimental to patient safety. Once identified, such practices could be examined more carefully, and refined, altered, or eliminated. Individual review of a database of such large volume would be infeasible without electronic means (Edsall, Jones, \& Smith, 1992).

Such queries have been shown to reveal potentially detrimental events occurring during the course of anesthesia delivery, with the use of electronic review illustrated to be more sensitive than the practice of voluntary reporting of such incidents. Sanborn et al (1996) compared deviations from normal and expected values of vital signs (heart rate, blood pressure, oxygen saturation, and temperature) voluntarily reported by anesthesia providers and the same deviations discovered by computerized searching software of anesthesia records collected by an AIMS. The electronic scans revealed 434 "incidents," 
of which only 18 were voluntarily reported by anesthesia providers (K. V. Sanborn, Castro, Kuroda, \& Thys, 1996a).

Identification of errors or potential complications is only the first step in a series of measures that move medical care toward improvement. Once a problem has been identified, the factors that contribute to the negative outcome must be evaluated, with a goal of prevention of complications. AIMS have been utilized to assist the anticipation of potential complications, as illustrated by the use of AIMS for prediction of the need for antiemetic treatment in a postanesthesia care unit (PACU) (Junger et al., 2001). AIMS have also been utilized for the identification of more serious complications that may develop over the course of an anesthetic, such as the prediction of hypoxia during onelung ventilation (Sticher et al., 2002), or for the need for inotropic support for patients undergoing cardiac surgery (Jost et al., 2003). Such predictions were accomplished through complex software analysis of large consolidations of many anesthetic records over many years, made possible by the electronic collection and storage of this data.

Further research supported the development of such large databases through use of AIMS. Examination of the physiologic effects of anesthetic agents has been examined, with suggestions for practice guidelines that may result in enhanced patient safety. Specific analysis of the physiologic effects of selected induction agents was categorized by ASA classification of patients, with the outcome of post-induction hypotension measured (Benson et al., 2000). After analysis of over 8,000 anesthetic records, of the three agents studied, propofol was found to produce the greatest reduction in blood pressure, followed by Thiopental, and etomidate produced the least hypotension. 
Further division of patients by ASA classification revealed that hypotension was greater in those patients with a classification of III or higher, and least with those of II or lower. These findings were consistent with literature specifically analyzing the physiologic effects of these agents, leading the authors to conclude that data analysis of computerized records was valid and reliable (Benson et al., 2000).

Analysis of electronic records may also be condensed to focus on the outcomes of specific anesthesia procedures. The incidence of severe bradycardia or asystole following spinal or epidural anesthesia administration was examined by the use of an AIMS database in 2003. Over 57,000 records were analyzed, with cases that involved spinal or epidural anesthesia associated with general anesthesia eliminated from analysis. Of the resulting 6,663 cases, 677 recorded bradycardia following administration of the central neuraxial block, and 46 cases were found to have severe bradycardia defined as a heart rate less than 40 beats per minute (bpm). Results of the data analysis revealed that an initial heart rate of less than $60 \mathrm{bpm}$ and male gender were most predictive of severe bradycardia following neuraxial blockade, with spinal anesthesia resulting in a higher incidence of bradycardia than epidural anesthesia administration. These findings were presented to enable anesthesia practitioners to recognize the potential for severe bradycardia in select patients, allowing for alternate anesthesia plans or pretreatment with agents to increase heart rate in susceptible patients. Development of such results served to establish the benefit of an AIMS database to significantly increase the speed and accuracy of analysis of a large volume of records (Lesser, Sanborn, Valskys, \& Kuroda, 2003). 
Development of results such as those presented by Lesser, et al (2003) and others (Egger Halbeis \& Epstein, 2008) encouraged the development of "decision support systems (DSS)s" to identify patients at risk of anesthetic complications and make appropriate adjustments in anesthesia administration and planning to prevent negative outcomes. Limitations of such a system were illustrated by Epstein et al (2009). Delay in entry of automated records into a consolidated database could affect the results of targeted queries that could be influenced by the absence of such late records. Other potential sources of error included the absence of entries, a delay in processing the record from the workstation to the central database, the rounding of timing of events by the AIMS, or by the lack of synchronization of clocks throughout the operating theater. When relying on queries to a large database generated by an AIMS, users were cautioned to take delays and latency of entries into account prior to making policy decisions based solely on this data (Epstein, Dexter, Ehrenfeld, \& Sandberg, 2009).

\section{From the Past to the Future}

Initially, the anesthetic record was intended to provide a document of anesthetic care that could be reviewed to determine best practices and improve patient safety (Beecher, 1920). From the time of these early records, efforts to streamline the process through electronic means have been explored, from the initial device by McKesson (1934) which faded in to obscurity, to efforts with early computers, as illustrated by Drui (1973). Acceptance of the process of automation of the anesthetic record has not been uniform, as discovered by Block, at different times and at two separate institutions (F. E. Block Jr. et al., 1985) (F. E. Block Jr, Reynolds, \& McDonald, 1998). 
Many studies have attempted to define the superiority of AIMS or MERS, through the examination of vigilance, accuracy, completeness, enhanced capture of billing elements and cost containment, medico-legal protection of anesthesia practitioners, and quality improvement (Allard et al., 1995; Kay \& Neal, 1986; Loeb, 1994; N. S. Paget et al., 1981a; Thrush, 1992; Weinger et al., 1997; Yablok, 1990; Zollinger et al., 1977). Beyond research, opinions have fanned the flames of controversy based upon experiences and assumptions that may or may not be based on fact (Lees, 1985; T. N. Noel, 1986; K. R. Noel, 1991; Rosen \& Rosenzweig, 1985a; Saunders, 1990). The area of enhanced legibility of an anesthetic record produced by an AIMS has been well accepted (F. E. Block Jr., 1991; Driscoll, Columbia, \& Peterfreund, 2007; Edsall et al., 1993; Hogan \& Wagner, 1997; Lerou et al., 1988; Logas et al., 1987; Zollinger et al., 1977). The benefits of an AIMS for the purposes of quality improvement and enhanced capture of billing elements have also been well received by the anesthesia community (Benson et al., 2000; Egger Halbeis \& Epstein, 2008; Jost et al., 2003; Lubarsky et al., 1996; Lubarsky et al., 1997; O'Sullivan et al., 2007; A. Petry, Gockel, \& Wulf, 1993b; Reich et al., 2005; K. V. Sanborn et al., 1996a).

Other elements of the anesthetic record have proven to be more controversial. The concepts of accuracy and vigilance have produced strong and conflicting opinions (Lees, 1985; T. N. Noel, 1986; Rosen \& Rosenzweig, 1985a; Rosen \& Rosenzweig, 1985b; Rosen \& Rosenzweig, 1986; Saunders, 1990). The concern of artifact or erroneous data, especially when seen as a potential risk of exposure to medical malpractice suits has been an issue since the first inception of AIMS (Cook et al., 1989; 
Driscoll et al., 2007; Feldman, 2004; Gage, 1992; Gibbs, 1989a; Hogan \& Wagner, 1997; Lerou et al., 1988; Logas et al., 1987; Shibutani et al., 1990; Vigoda \& Lubarsky, 2006a; Vigoda \& Lubarsky, 2006b; Zollinger et al., 1977). The effect of the method of recordkeeping on vigilance of the anesthesia provider has been studied by multiple authors (N. S. Paget et al., 1981a) (Kay \& Neal, 1986) (Weinger \& Englund, 1990) (Loeb, 1994) (Woods et al., 1995)(Thrush, 1992; Weinger et al., 1997). Paget et al (1982) defined many of the impediments to vigilance in the anesthesia provider. Kay and Neal (1986) suggested that use of automated systems - automated blood pressure devices - may result in a decrease in vigilance in anesthesia providers. Weinger and Englund (1990) called for automation, but with "intelligence," in the form of alarms and visual alerts to call attention to deviation from normative values. Loeb (1995) attempted to directly study vigilance of anesthesia residents through the use of a "vigilance task" and a second anesthesia provider to act as a scribe. This research was criticized by Woods et al (1995), who indicated that a "scribe" was another human anesthesia provider who could participate in the delivery of anesthesia. The authors contended that a computerized recordkeeping system could not participate in anesthesia delivery, either through direct action or by approval of the actions of the subject. The author also failed to identify the possibility of an expectancy effect in the conduction of the vigilance task. Weinger et al (1997) also attempted to directly manipulate vigilance through the use of a vigilance task, in this case, a light that would illuminate until a button was pressed. As with Woods et al (1995), no mention was made of the possibility of an expectancy effect by the subjects to the vigilance task. Thrush (1992) examined accuracy of data recorded by anesthesia 
providers using AIMS and those using MERS. This research claimed definitive results in favor of superior accuracy of practitioners using an AIMS, yet was subject to many methodological flaws. The study population was very small in size (13 cases), and data was collected on widely dissimilar cases, and a disparate number of records were generated by the subjects. Four cases did not record data for the full time allotted, due to the need to emergently initiate cardiopulmonary bypass, indicating a significant difference in workload and task density, yet these cases were included in the results.

In spite of any controversy clouding adoption of AIMS, potential benefits of computerized systems have resulted in the call for increased adoption (Stoelting, 2001). Some authors feel that the widespread adoption of AIMS would be a part of the solution to the unacceptable rate of medical error illustrated by the landmark document, "To Err is Human," published by the Institute of Medicine in 1999 (Cooper, 2007). The advancement of computing technology, the effort to standardize medical terminology and electronic records, and the potential cost savings of consolidating all areas of medical care into a single system, all serve to support the claim that AIMS are "poised to revolutionize anesthesia care" (Levitan, 2008). The proponents of computerization appear to be overcoming the critics of AIMS, in that $44 \%$ of academic medical centers in the United States have implemented, or are in the process of implementing an AIMS as of 2008(Egger Halbeis et al., 2008). Critics claim some leverage by raising fears of the development of "fully automated anesthesia systems," such as the Canadian "McSleepy" (Shelley, 2008). Despite these fears, however, the United States government has strengthened the call for more advanced technology to be applied to the health care field, 
and specifically to medical records (Huslin, 2009). In 2005, a goal was set to have an electronic medical record in place for each citizen of the United States by the year 2014 (Huslin, 2009). While obstacles to this goal echo many of the concerns of anesthesia providers regarding AIMS, financial incentives for physicians, medical practices, and hospitals included in the recent $\$ 19$ billion economic stimulus package may serve to further increase adoption of computerized systems, including AIMS (Huslin, 2009).

\section{Trigger Films}

Following the initial assessment of accuracy of recall of anesthesia providers using each method of recordkeeping, a presentation of the benefits and limitations of each method will be provided by the use of a trigger film. Trigger films present a brief scenario that simulates an actual patient care incident. Designed to induce an audience response, the film is stopped to allow discussion of the issue portrayed.

Ber and Alroy (2001) describe their many years of experience with the use of trigger films in the education of medical students in Israel. The authors cite that the discussions "triggered" by the film also stimulate reflective thinking about ethical and behavioral aspects of the presentation, in addition to the objectives of the film. In producing the trigger film, the authors find that brief three to five minute scenarios should be filmed in an environment that is familiar to the audience. The authors indicate that discussions may become "very lively and often noisy" (Ber \& Alroy, 2001).

Hartland et al (2003) describe the use of trigger films as an educational approach to clinical anesthesia instructors. The authors use the traditional format described by Ber and Alroy (2001), with the addition of another brief film that offers one solution to the 
issue presented. This second film is presented after a period of discussion, and may serve to either stimulate further discussion or to refocus the group to the objectives of the lesson (W. Hartland, Biddle, \& Fallacaro, 2003).

Hartland (2003) made films in a facility designed to accurately simulate an actual operating room environment. Clinical instructors in New York, North Carolina, and Virginia were asked to describe their perceptions of the films in terms of content of the films, believability, and validity of the attributes of the clinical instructor represented. In addition, the clinical instructors were asked to rank the top three most valuable attributes of each scenario presented, and to provide feedback for improvement. The ranking of attributes of the clinical instructors were consistent with previous research by these authors on the qualities of an ideal clinical educator (W. Hartland Jr \& Londoner, 1997). Hartland (2003) indicated that the trigger film was an effective alternative to point-ofcare teaching in an operating room environment, an environment that is poorly designed for effective teaching. Trigger films may be used in an environment conducive to learning, with no risk of harm to an actual patient.

\section{Information Processing}

The field of cognitive psychology focuses on the ways in which humans process information. A review of information processing offers insight into the differences of vigilance, or "connectedness" to the patient's condition by anesthetists recording vital signs and parameters with an AIMS or with MERS. These differences in the processing of information from the moment of assessment of a vital sign or parameter into the memory and judgment of the anesthetist will serve as the framework of this research. 
The modal model of memory as proposed by Atkinson \& Shiffman in 1968 is composed of three stages of memory. Sensory memory refers to all stimuli that are received through the five senses (Atkinson \& Shiffrin, 1968). The capacity of sensory memory is vast, and may include the entire visual field, yet the duration of this memory is extremely brief, only 500 milliseconds (Sperling, 1960). Stimuli are perceived by one of two types of processing: bottom-up or data-driven processing, or top-down or conceptually driven processing. In bottom-up processing, data is perceived and directly enters the neural network of memory. In top-down processing, data stimulates previous experiences or memories to define perception and is linked with this stored information in memory (Robinson-Riegler \& Robinson-Riegler, 2004).

\section{Sensory Memory and Attention}

Atkinson \& Shiffrin (1968) proposed that all information that is perceived by humans is entered into a "sensory memory store." Visual stimuli have been shown to decay within 500 milliseconds, while auditory stimuli may linger up to 2 minutes from the time of presentation. Movement of stimuli into short term memory requires attention, either voluntarily or involuntarily. Through voluntary control, attention to certain stimuli is proposed as a "gateway" into short term memory storage. Stimuli may be filtered, allowing only the most important or significant information to pass into short term memory. Still, some stimuli may be attended to through involuntary means, as evidenced by the "cocktail party phenomenon" proposed by Treisman (1960). This phenomenon occurs when humans attend to stimuli, such as a spoken name by a stranger at a cocktail party, even when they are not actively paying attention to the speaker (Treisman, 1960). 
The most widely accepted theory of attention is that of attenuation, in which stimuli are not entirely blocked from sensory memory, but rather the signals are "turned down" or attenuated, to partially filter the vast amount of information presented (Robinson-Riegler \& Robinson-Riegler, 2004). The theory of attenuation is supported by studies of the neurologic system, as noted by Driver (2001). Attention to information depends largely on the context of the situation or the importance of the perceived stimuli, yet unconscious attention may occur, as the exception rather than the rule (Driver, 2001). In an operating room environment, many separate sources of both auditory and visual stimuli are presented simultaneously.

Anesthesia monitors continuously display data on the condition of the patient, through numeric or graphical display of vital signs or other parameters, producing auditory tones and occasionally alarms to alert the provider to extreme values. The surgeon speaks with his assistant or nurses, asking for instruments or procedures, and occasionally to the anesthesia provider, seeking information about the patient's condition or to ask for changes in patient position. The anesthetist perceives all these stimuli, processing important information, disregarding irrelevant or distracting stimuli, and responding to queries or requests, perhaps without being consciously aware of attending to any such information. Much of the attenuation of this data takes place through topdown processing, as the anesthetist links current vital signs and parameters to previously determined normative values, and is influenced by the volume of task demands of the anesthetic case. 


\section{Automatic Memory}

Over time, individuals develop some mental processes that become automatic (Posner \& Snyder, 1975). After repeated exposure to the same set of stimuli, information may be processed without conscious attention or awareness. Such processes are simple to conduct when task demands are high, as they require very little mental effort and consume only a small amount of memory capacity. These automatic memory tasks may be difficult to consciously define; difficult to explain, but simple to demonstrate. Many of the tasks of the anesthetist (intubation, regional anesthesia administration) may become automatic over time, allowing for additional mental capacity for multiple tasks to be performed at once (awareness of vital signs during intubation or extubation).

To develop automaticity, Schneider \& Shiffron (1977) proposed that individuals conduct a visual search of their environment. When target items have a consistent location, the capacity of the individual's memory is unlimited, and recall tasks improve with practice. When items have an inconsistent location, or the location of these items is changed from one exposure to the next, the capacity of memory is significantly limited, and performance does not improve over time (Schneider \& Shiffrin, 1977). Given this theory, anesthetists who work in a familiar environment will have a greater capacity of memory than those who are newly introduced to an environment or procedure. Through repeated exposure in a consistent environment, memory will improve. Logan (1988) described this process as an instance based view. While initial performance of a task requires a significant amount of mental effort, with repeated experiences with the same task, performance may become automatic, as many "instances" of the activity are stored 
in memory. Initially, the individual may be able to readily describe the task in a detailed and step-wise fashion. With practice, the ability to describe the steps of the task may diminish, but actual performance of the skill may improve, reflecting the presence of automatic memory (Logan, 1988). Automaticity gives rise to errors, known as "action slips." The term "absentmindedness" is a more common term used to describe these action slips, which occur, literally when the individual "isn't thinking." Action slips become more common when attention is reduced by interference, such as when the individual is tired, stressed, or overloaded with multiple tasks - common occurrences in anesthesia.

\section{Short Term Memory}

Stimuli to which humans give attention are transferred into short term memory, which has a smaller capacity, but a significantly longer duration. Memories contained within short term memory also fade quickly, with up to $90 \%$ of information lost within 20 seconds (Peterson \& Peterson, 1959). The short term memory may also have a very limited capacity, with $7 \pm 2$ digits cited as a "magic number," and is the basis of the seven digit telephone number (Miller, 1956).

Encoding of information into long term memory involves a process known as “chunking," or categorizing information into smaller groups. Remembering a 7 digit telephone number is easier than a 10 digit number that includes the area code. To aid memory, individuals divide the ten digit number into two "chunks," the area code (3 digits) and the telephone number (7 digits). Note-taking during lecture is considered a form of "chunking” (Robinson-Riegler \& Robinson-Riegler, 2004). Handwritten 
anesthesia records would be considered a form of note-taking, or chunking, of patient information. The method of presentation of information to the subject and the individual's knowledge base significantly affect the quality of chunking. The skilled memory theory proposes that individuals with significant expertise in a given topic may directly encode information about this topic directly into long term memory. This direct link is enabled by a "rich recoding scheme," that links new information to previous memories of similar information (top-down processing) (Robinson-Riegler \& RobinsonRiegler, 2004). Word length has a inverse relationship to memory, with longer words more difficult to encode. Phonological similarities or words that sound similar are also more difficult to encode and recall. Information that is negatively impacted by such presentation is more likely to result in forgetting the data than the loss of properly encoded material over time, or decay. Interference may also take the form of displacement, when new information dislodges previously stored data. Interference may also overwrite previously stored information.

\section{Working Memory}

Currently, many psychologists refer to memory with the term working memory. Working memory, as described by Baddeley and Hitch (1974), consists of a "central executive" that guides the processing of information from two subgroups, the visualspatial sketchpad and the articulating loop. The visual-spatial sketchpad processes information from images and spatial relationships, while the articulating loop processes auditory or spoken (phonological) data (including subvocal rehearsal - silently repeating a word or digit - rehearsing the data to improve memory). These two loops do not 
interfere with each other, and are managed by the central executive. The central executive is a system of higher processing, dealing with reasoning, comprehension and problem-solving. This system acts as a "gatekeeper," processing information to determine the merit of the data prior to filing in long term memory (Baddeley \& Hitch, 1974). The central executive also may act as a "capacity allocator," switching the capacity of either the visual-spatial sketchpad or the articulating loop dependent upon the needs of the environment or presentation of information.

Whether processed by the articulatory loop or the visual-spatial sketchpad, information is often identified and classified prior to more advanced processing. The process of identification may be subdivided into patterns (letters or numbers), objects (physical items in the environment), and faces. For the purposes of this research, an understanding of pattern recognition is important.

Identification of patterns involves the recognition of concepts - a predetermined representation of a particular pattern, a form of "mental shorthand." (Robinson-Riegler \& Robinson-Riegler, 2004). Categories are subgroups of concepts, and contain many, smaller elements of the larger concept. To the anesthetist, the concept of the patient as stable may be divided into many categories of vital signs, parameters, and conditions. Vital signs may be categorized as normal when current values are compared with previously encoded values that have been indicated by both education and experience to be consistent with homeostasis.

Categorization has been researched extensively by cognitive psychologists, with the explanation-based approach perhaps the most representative of information 
processing for the anesthetist. In the explanation-based approach, the individual compares presented values with personal judgments of similarity to members of the respective category. These features are created through past education and experiences with similar members of a given category (Robinson-Riegler \& Robinson-Riegler, 2004). In this case, a value of 80 for heart rate is compared to previously learned and experienced normative values for the category of "acceptable" heart rate, values that may differ from patient to patient and vary by patient condition, reflecting context sensitivity. The explanation-based approach accounts for all such variables (age, co-existing disease, current condition or disease-state), to compare the present value with those values that, based on the judgment of the anesthetist, would be expected in the category of "acceptable," as one aspect of the concept, "stable."

\section{Long Term Memory}

Memories stored in the short term memory bank that are regarded with particular importance may be moved into long term memory. Authors of previous research have proposed that there are two distinct types of long term memories, episodic and semantic memory (Tulving, 1972). Episodic memories stem from events that the individual has actually experienced. Semantic memories are not actually experienced, but are learned and accepted as fact. Anesthetists may have knowledge of the signs and symptoms of a given event, such as malignant hyperthermia, even when they have never actually experienced a patient suffering from this particular disorder. Retrieval of each of these types of memory involves different processes. Episodic memories are associated with strong recollection, often recalling many aspects of the event, including the time, place, 
surroundings, and even emotions felt at the time. Semantic memory is simply the recall of a specific fact. Over time, episodic memories may fade, and have been found to be more prone to forget as compared with semantic memories. Many researchers believe that these two types of memory are not separate systems, but are more likely aspects of a more unified mental process ((Robinson-Riegler \& Robinson-Riegler, 2004)

\section{Encoding, Storage, and Retrieval}

Melton (1963) proposed this simple explanation of the processing of information. Stimuli must be encoded into a specific storage area, either short- or long-term memory. Once stored, information must be retrieved by some process in order to be useful. Often, the process of retrieval fails, or partially fails - the "tip of the tongue" phenomenon. In order to be properly encoded, several strategies must be employed. As previously stated, individuals must pay attention to a stimulus in order to begin the process of encoding. Once attended, information deemed important may be repeated, or rehearsed, to more definitively encode data into long term memory. The process of massed repetition involves a concentrated effort of repetition over a short span of time, such as practicing multiplication tables, or attending several lectures on the same topic on a given day. Distributed repetition involves repetition over a longer period of time, such as attending another lecture as a means to study material presented days or weeks before. Of the two forms of repetition, distributed repetition has shown greater benefit to recall when compared with massed repetition, originally termed the "spacing effect" (Melton, 1963).

Rehearsal is a form of mentally practicing information to enhance storage. While repetition refers to repeated presentations of information, rehearsal refers to an internal 
review of the information previously received. Handwritten note-taking is a form of rehearsal, but rehearsal may also occur by simply thinking about the information, mentally repeating the information. This form of repetition is known as maintenance rehearsal, and merely serves to retain the data in consciousness. The effectiveness of this form of rehearsal depends on the task of retrieval from long-term memory. If asked to recall a specific word, number, or definition, as with a short answer exam, maintenance rehearsal is not very effective, as there are no "hints" to aid retrieval of the data. Performance on a task of recognition, as with a multiple choice exam, is greatly enhanced by maintenance rehearsal. In this task, the correct answers are given, and simply need to be recognized. A more effective method of encoding information is elaborative rehearsal, where the information is not simply repeated (or re-written), but is redefined or elaborated upon, giving greater meaning to the presentation. Connecting the presented information to pre-existing data already stored in memory enhances the likelihood of recall, by either task of retrieval (Robinson-Riegler \& Robinson-Riegler, 2004).

The use of visual imagery, as in a trigger film presentation, may also strengthen encoding of information into long term memory. Paivio (1971) proposed the dual coding theory, which described two systems of memory. One system encodes concrete images or symbols visualized by the subject, while the second system is one of verbal representations of an event or concept. More intangible concepts may be encoded only through the verbal system, more concrete concepts may be encoded by both systems, enhancing future recall (Paivio, 1971). 
Armed with an understanding of information processing, this study will attempt to explore the nuances of encoding of patient data by anesthetists using AIMS and those using MERS. Each type of recordkeeping may utilize some form of either "bottom-up" or "top-down" processing in order to process the many stimuli presented simultaneously in an operating room environment. Certainly some form of attenuation takes place, as each anesthetist must both consciously and unconsciously filter the many streams of information competing for memory capacity, and still retain quick responses to queries, requests, or changes in patient condition. With practice, anesthetists may develop automatic memories, and recall may be enhanced by greater experience with the method of recordkeeping employed. The role of chunking of information may explain the ability of anesthetists to recall patient data, as each vital sign or parameter fall well within the "magic number" of $7 \pm 2$ digits. Anesthetists may also employ concepts and categories of information, applying an explanation-based approach of processing the vital signs and parameters presented by each individual patient to the store of previous experiences and knowledge base that forms the foundation of clinical judgment. The repetition of information presented in a short span of time may aid recall, yet differences in rehearsal, either by handwritten records, or by subvocalizing visual displays or even auditory alarms, may account for differences in recall. Anesthetists may unconsciously employ methods to enhance encoding, aided by the "central executive" and the switching of resources between an articulatory loop and a visual-spatial sketchpad.

Information processing offers potential explanations for both the proposed benefits of computerized recordkeeping, as well as the perceived importance of 
handwritten records to maintain "connectedness" to patient condition and to the course of a safe anesthetic. This study proposes to examine memory through a simple task of recall of vital signs and parameters of importance to each anesthetic case. Anesthetists who practice in facilities utilizing AIMS will be compared to anesthetists practicing in facilities using MERS, to assess any differences in recall accuracy. Any differences will be further explored by comparing any potential influence of age, gender, years of experience as a CRNA, as well as years of experience with each method of recordkeeping, guided by the concepts of information processing.

Based upon Paivio's dual-coding theory, a trigger film will be developed to demonstrate beneficial and detrimental effects of each method of recordkeeping. By presenting simulated operating room scenarios, both visual and verbal components of encoding will be utilized to enhance storage of this information into long term memory. Once stored, this dual-coded information should be readily recalled to alter the recordkeeping practices of anesthetists, potentially enhancing the beneficial aspects and reducing the limitations of their respective method of recordkeeping. Any such changes in practice will be assessed through the application of the proposed instrument to measure accuracy of recall, both in subjects who have viewed the trigger film presentation, as well as those who did not, to attempt to measure differences among the groups. 


\section{CHAPTER THREE: METHODOLOGY}

Certified Registered Nurse Anesthetists (CRNAs) administer more than 30 million anesthetics each year(American Association of Nurse Anesthetists, 2008). Each of these anesthetics is accompanied by an account of the events of the surgery and anesthetic, vital signs and parameters of the patient, and a record of all medications administered. These records are generated by either manual entry of data into a standardized form developed by the institution or anesthesia group, or through a computer-generated record. Anesthesia information management systems (AIMS) are reported to be utilized in five to ten percent of hospitals across the United States. One manufacturer of anesthesia delivery systems and monitors, Drager Medical, states that the Innovian ${ }^{\circledR}$ AIMS is in place in over 130 hospitals across the United States, and is used by more than 7,000 practitioners world-wide (Innovian anesthesia - your competitive advantage. 2008).

Through both research and opinion, the anesthesia community has sought to define the benefits of AIMS while controlling any potential limitations, real or imagined. Studies of the accuracy and impact on vigilance of an anesthesia provider using an AIMS as compared to traditional handwritten records have claimed definitive results, yet were hindered by methodological flaws (Thrush, 1992)(Loeb, 1994). Benefits of AIMS have been shown to be less controversial. Utilization of large databases of patient information has enhanced the quality assurance process, particularly through improvements in the identification of critical incidents (K. V. Sanborn, Castro, Kuroda, \& Thys, 1996b). 
Identification of potential complications through the use of such databases may result in changes in practice, or initiation of practice guidelines designed to improve patient safety (Benson et al., 2000). Development of practice guidelines has also been suggested as a way to enhance efficiency of anesthesia delivery, resulting in significant cost savings to the anesthesia group and hospital (Lubarsky et al., 1997). Enhanced capture of billing elements has also been suggested as a welcome benefit to use of an AIMS, and such financial incentives have been offered as a "talking point," as justification to hospital administrators that the initial cost of implementation will result in ultimate cost savings in addition to patient safety (O'Sullivan et al., 2007). At the present time, even government legislation has proposed incentives for the development and implementation of electronic medical recording technology, including AIMS (Huslin, 2009).

\section{Relevance to Nurse Anesthesia}

For each anesthetic administered, a detailed record is generated that includes vital signs, medications, and events of the surgery or procedure. Patients are monitored according to standards published by both the American Society of Anesthesiologists and the American Association of Nurse Anesthetists (AANA). These standards detail the vital signs and parameters that must be recorded by an anesthesia provider to document the monitoring of oxygenation, ventilation, circulation, and temperature (AANA - scope and standards for nurse anesthesia practice.2007; ASA, 2005). Since the development of the standards, the overwhelming majority of anesthesia providers have recorded this data 
manually on pre-printed forms. This form of manual entry recordkeeping (MERS)

persists in more than 90 percent of anesthesia practices (Levitan, 2008).

\section{Statement of Purpose}

The primary purpose of this research was to analyze the practitioner's accuracy in recalling patient data when using each method of recordkeeping. A second purpose of this research was to illustrate the effectiveness of a trigger film that details the benefits and limitations of both recordkeeping methods on the subsequent recall accuracy of anesthesia providers. A summary of independent variables, dependent variables, and covariables is provided in Table 3 .

Table 3: List of Variables

\begin{tabular}{|c|c|c|c|c|}
\hline \multicolumn{2}{|r|}{ Research Question } & $\begin{array}{c}\text { Independent } \\
\text { Variable }\end{array}$ & Dependent Variables & Co-Variables \\
\hline$\overline{1 .}$ & $\begin{array}{l}\text { Do anesthesia providers } \\
\text { using AIMS } \\
\text { recordkeeping } \\
\text { demonstrate equivalent } \\
\text { recall of specific patient } \\
\text { variables, as compared } \\
\text { with anesthesia provider } \\
\text { using a MERS? }\end{array}$ & $\begin{array}{l}\text { Method of } \\
\text { Recordkeeping } \\
\text { - MERS vs. } \\
\text { AIMS }\end{array}$ & $\begin{array}{ll} & \text { Accuracy of: } \\
\text { 1. Highest HR } \\
\text { 2. Lowest HR } \\
\text { 3. Highest SBP } \\
\text { 4. Lowest SBP } \\
\text { 5. Highest ETCO } \text { HCO }_{2} \\
\text { 6. Lowest ETCO } \\
\text { 7. Highest IP } \\
\text { 8. Total Fluid Volume }\end{array}$ & $\begin{array}{l}\text { 1. Age of provider } \\
\text { 2. Gender of provider } \\
\text { 3. Years of experience as a } \\
\text { CRNA } \\
\text { 4. Years of experience } \\
\text { with recordkeeping } \\
\text { method }\end{array}$ \\
\hline 2. & $\begin{array}{l}\text { Does the presentation } \\
\text { of the benefits and } \\
\text { limitations of } \\
\text { recordkeeping } \\
\text { practices by trigger } \\
\text { film influence the } \\
\text { recall accuracy of } \\
\text { specific patient } \\
\text { variables by anesthesia } \\
\text { providers using AIMS } \\
\text { or MERS? }\end{array}$ & $\begin{array}{l}\text { 1. Between } \\
\text { subjects - } \\
\text { Instruction vs. } \\
\text { No instruction } \\
\text { 2. Within subjects } \\
\text { - method of } \\
\text { recordkeeping - } \\
\text { MERS vs. } \\
\text { AIMS }\end{array}$ & $\begin{array}{ll} & \text { Accuracy of: } \\
\text { 1. } & \text { Highest HR } \\
\text { 2. } & \text { Lowest HR } \\
\text { 3. } & \text { Highest SBP } \\
\text { 4. } & \text { Lowest SBP } \\
\text { 5. } & \text { Highest ETCO } \mathrm{ECO}_{2} \\
\text { 6. } & \text { Lowest ETCO } \\
\text { 7. } & \text { Highest IP } \\
\text { 8. } & \text { Total Fluid Volume }\end{array}$ & $\begin{array}{l}\text { 1. Age of provider } \\
\text { 2. Gender of provider } \\
\text { 3. Years of experience as a } \\
\text { CRNA } \\
\text { 4. Years of experience } \\
\text { with recordkeeping } \\
\text { method }\end{array}$ \\
\hline
\end{tabular}

This project sought to answer two research questions: 
- Do anesthesia providers using AIMS recordkeeping demonstrate equivalent recall accuracy of specific patient variables, as compared with anesthesia providers using a MERS?

- Does the presentation of the benefits and limitations of recordkeeping practices by trigger film influence the recall accuracy of specific patient variables by anesthesia providers using AIMS or MERS?

To answer these research questions, the following hypotheses were be analyzed:

$\mathrm{H}_{1} \quad$ For each anesthetic case, there will be no difference in the accuracy of recall of specific patient variables recorded by an anesthesia provider using AIMS or an anesthesia provider using a MERS.

$\mathrm{H}_{2} \quad$ For each anesthetic case, there will be no difference in the accuracy of recall of specific patient variables recorded by an anesthesia provider who has attended a trigger film presentation on the benefits and limitations of recordkeeping methods than a provider that has not received such instruction.

\section{Population and Recruitment}

This research drew a sample of CRNAs from the population of more than 200 CRNAs practicing at the study site facilities in Virginia. CRNAs assigned to each facility on the day of evaluation were asked to complete a simple questionnaire (See Appendix A). A trained observer provided each practitioner with brief relief from patient care, after constant attendance of the patient for a minimum of 30 minutes of anesthesia 
care during the maintenance phase of the case. The CRNA assigned to the case was asked to turn away from the monitors and complete the questionnaire. The observer documented values directly from the trend data recorded in the patient monitor. Cases were limited to those utilizing general endotracheal anesthesia and mechanical ventilation, to ensure homogeneity of the sample and collection of data in all categories.

Four centers were included in the project, selected by similarities of number of operating rooms, case load, and number of CRNA providers. These facilities are listed in Table 1. Written permission was been obtained from all sites that participated in this research.

\section{Instrument Development}

A standardized instrument was developed (See Appendix 1) based upon previous research (Yablok, 1990; Allard et al, 1995), to evaluate the accuracy of anesthesia providers' recall when recordkeeping with AIMS or MERS. Eight questions were developed, asking practitioners to recall specific data for highest and lowest heart rate (HR), highest and lowest systolic blood pressure (SBP), highest and lowest end-tidal carbon dioxide $\left(\mathrm{ETCO}_{2}\right)$, total fluid volume administered, and highest inspiratory pressure (IP), all within the last thirty minutes of the case. Use of the 30 minute time interval was selected to ensure measurement of recall during part of the maintenance phase of the anesthetic, a documented time of reduced workload (Allard et al., 1995; Weinger et al., 1997; Weinger, Reddy, \& Slagle, 2004). A team of experts consisting of a CRNA with a PhD in epidemiology, an anesthesiologist responsible for the selection and implementation of an AIMS at the VCU Medical Center, a professor and 
anesthesiologist with expertise in AIMS development and implementation, a nurse practitioner with expertise in the field of information technology, and a professor of cognitive psychology, reviewed the instrument and justifications for the selected questions to establish face validity.

\section{Justification / Selection of Parameters}

Previous research indicated several vital signs and parameters as variables of interest in the measurement of accurate recall by anesthesia providers (Yablok, 1990; Allard et al, 1995). Heart rate has a wide range of acceptable values, allowing practitioners to note this value without frequent need for intervention (Morgan, Mikhail, \& Murray, 2005). Heart rate is indicated on anesthesia monitors in both a large, bold, numerical value as well as by a continuous graphic display of two electrocardiograph (ECG) leads (see Figure 1).

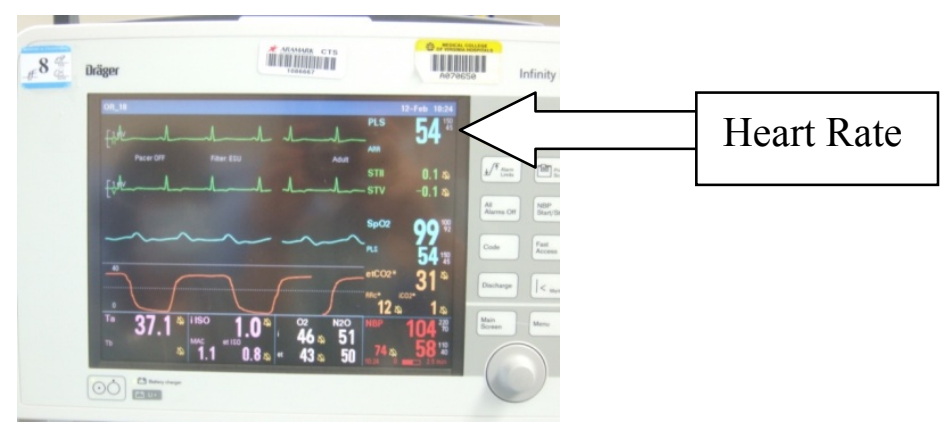

Figure 1: Display of Heart Rate

Systolic and diastolic blood pressures are indicated in a large, bold, numerical value when recorded by a non-invasive blood pressure cuff, as well as by a continuous graphic display of a waveform accompanied by a large, bold, numerical value when recorded by an invasive arterial catheter, as shown in Figure 2. 


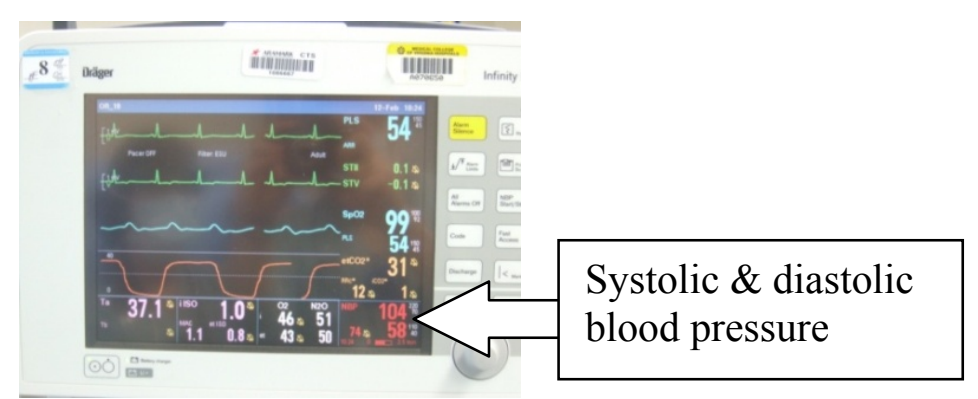

Figure 2: Display of Blood Pressure

Systolic blood pressure has a wide range of acceptable values, allowing practitioners to note this value without frequent need for intervention during the maintenance phase of the anesthetic (Morgan et al., 2005). Systolic blood pressures have been identified as variable when recorded by computerized systems that directly record each value. Manually entered records have been noted to produce inaccuracies known as “smoothing” (F. E. Block Jr., 1991; Cook et al., 1989; Lerou et al., 1988; Shibutani et al., 1990). Cases with arterial blood pressure readings were excluded, as beat-to-beat measurement may result in differences in averaging in five minute trend data.

End-tidal carbon dioxide values are indicated in both a large, bold, numerical value as well as by a continuous graphic display of a capnography waveform (See Figure 3). $\mathrm{ETCO}_{2}$ has a narrow range of acceptable values, which vary based upon changes in ventilation frequency and tidal volume delivered (Morgan et al., 2005). Practitioners observe $\mathrm{ETCO}_{2}$ values when verifying placement during general anesthesia (GA) with an endotracheal tube (ETT) or Laryngeal Mask Airway (LMA), when making initial ventilator settings during GA with an ETT, as well as at variable intervals throughout the anesthetic case to determine if minor adjustments are needed to reduce the $\mathrm{CO}_{2}$ level to prevent spontaneous respirations or respiratory alkalosis, or to reduce the $\mathrm{CO}_{2}$ level to 


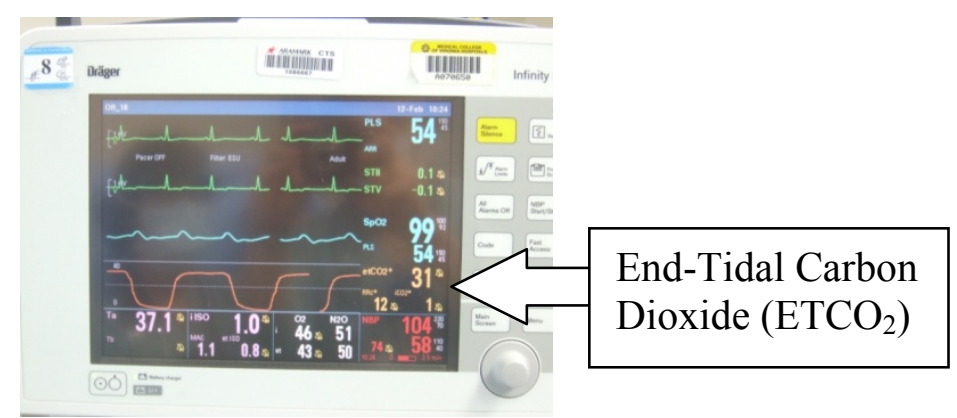

Figure 3: Display of End-Tidal Carbon Dioxide

prevent respiratory acidosis (Morgan et al., 2005)(ASA House of Delegates, 2005).

Practitioners observe $\mathrm{ETCO}_{2}$ values intermittently, as a qualitative measure of adequacy

of spontaneous respiration, during Monitored Anesthesia Care (MAC), or regional anesthesia with sedation. Continuous $\mathrm{ETCO}_{2}$ monitoring is required for any case that necessitates artificial airway support (AANA - scope and standards for nurse anesthesia practice.2007; ASA House of Delegates, 2005).

Inspiratory pressure (IP) values are indicated by a small, single color, numerical value on the ventilation screen of the anesthesia machine, and by the position of the needle on the pressure gauge of the ventilator circuit (See Figures 4 and 5). IP has a wide range of acceptable values, which vary based upon changes in lung compliance, pathologic disease states, and tidal volume delivered, with an accepted maximum of less than $50 \mathrm{cmH}_{2} \mathrm{O}$ with endotracheal intubation, and less than $20 \mathrm{cmH}_{2} \mathrm{O}$ for mask or LMA ventilation. IP higher than $50 \mathrm{cmH}_{2} \mathrm{O}$ may result in barotrauma, and IP higher than 20 $\mathrm{cmH}_{2} \mathrm{O}$ with a face mask or LMA may produce an increased risk of aspiration (Morgan et al., 2005). Practitioners observe IP values when verifying placement during general anesthesia (GA) with an endotracheal tube (ETT) or Laryngeal Mask 


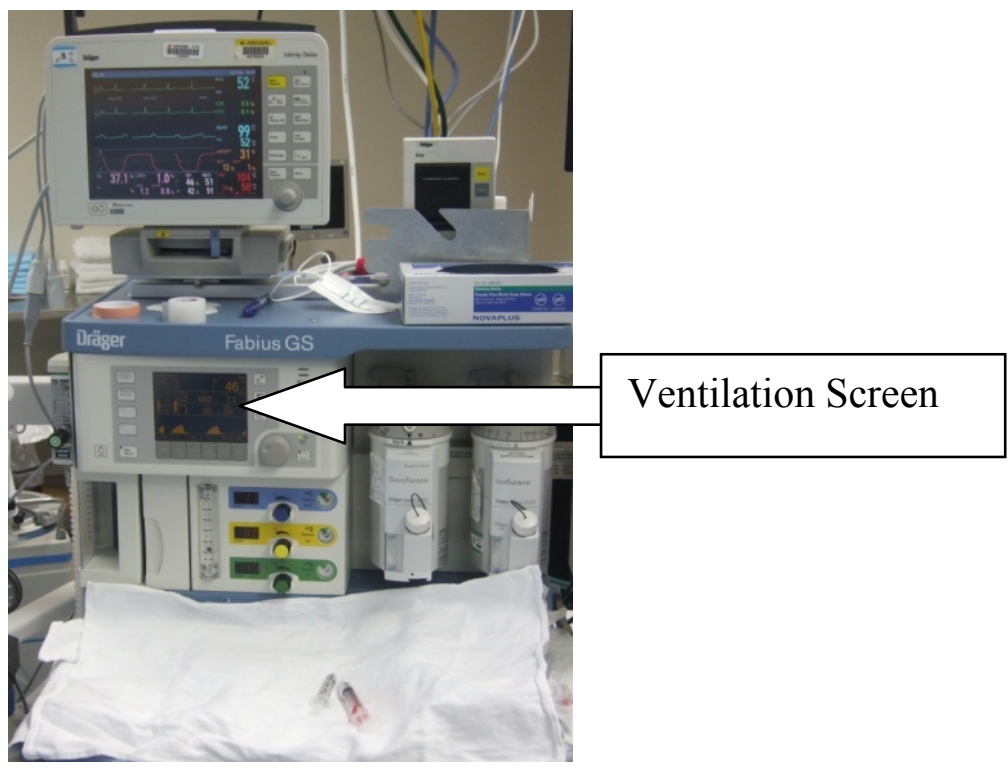

Figure 4: Display of Ventilation Screen - North American Dräger Fabius Model

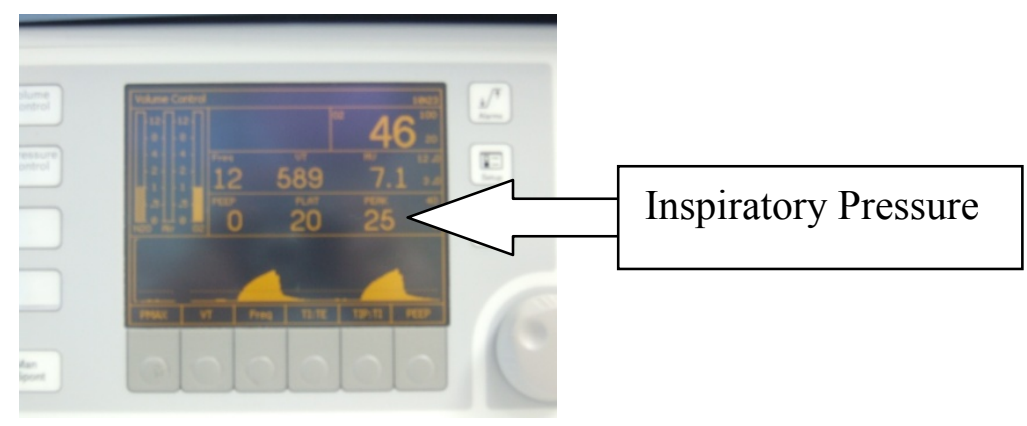

Figure 5: Display of Inspiratory Pressure - American Dräger Fabius Model

Airway (LMA), when making initial ventilator settings during GA with an ETT, as well as at variable intervals throughout the anesthetic case to determine if minor adjustments are needed to reduce the IP in the event of changes in lung compliance, or to detect an obstruction to ventilation or an accidental disconnection from the anesthesia circuit (Morgan et al., 2005)(ASA House of Delegates, 2005).

Fluid volume deficits are replaced by anesthesia practitioners in each anesthetic case, to varying degrees, depending upon the type and location of surgery, length of time of 
NPO (Latin: nil per os - nothing by mouth) or "fasting" status of the patient, and patient weight (Morgan et al., 2005). Replacement and maintenance of fluid volume is an integral part of each anesthetic, involving selection of type and volume of fluid administered (AANA - scope and standards for nurse anesthesia practice.2007). Notation of fluid volume must be entered manually by the practitioner using both computerized and manual entry recordkeeping systems.

\section{Frequency of Recording}

Practitioners using MERS document heart rate and systolic blood pressure every five minutes. $\mathrm{ETCO}_{2}$ and IP are recorded manually every fifteen minutes, while the frequency of fluid volume notation varies widely, dependent upon the individual practitioner and the length of the case. These time intervals adhere to the recordkeeping standards of the AANA \& ASA (AANA - scope and standards for nurse anesthesia practice.2007; ASA House of Delegates, 2005).

AIMS record vital signs and parameters at variable intervals, with systems capable of recording values every six seconds (Innovian anesthesia - your competitive advantage.2008). Frequency of graphical and/or numeric displays of vital signs and parameters (HR, SBP, ETCO 2 , IP) may be set by each individual institution, and usually follow the standards of the AANA and/or ASA. As with MERS, the frequency of recording fluid volume varies by institution and practitioner, and must be entered manually through a user interface, such as a keyboard. 


\section{Measurement of Accuracy}

Determination of accuracy of reported values was determined through a review of the relevant literature. Yablok (1990), in an abstract, studied the accuracy of recall of anesthesia providers over eight vital signs and parameters. The author established a value of \pm 9 millimeters of mercury ( $\mathrm{mmHg}$ ) for accuracy in determination of systolic blood pressure, \pm 9 beats per minute (bpm) for accuracy of recall of heart rate, \pm 4 centimeters of water pressure $\left(\mathrm{cmH}_{2} \mathrm{O}\right)$ for accuracy of peak inspiratory pressure (PIP), and \pm 3 mmHg for accuracy of recall of $\mathrm{ETCO}_{2}$ (Yablok, 1990). Allard et al (1995) also researched accuracy of recall of vital signs and parameters, with identical ranges for accuracy. Gravenstein et al (1989) used a dog model to determine changes in vital signs when hypotension, hypoxia, or hypercapnia was induced. The authors found that changes in SBP greater than $10 \mathrm{mmHg}$ required attention or intervention from the anesthesia provider. Intervention was also determined to be necessary for changes in $\mathrm{ETCO}_{2}$ greater than $10 \mathrm{mmHg}$, and for changes of greater than $10 \%$ in hemoglobin oxygen saturation $\left(\mathrm{SpO}_{2}\right)$ (Gravenstein et al., 1989). Lerou (1988) studied accuracy of computerized and handwritten records, using the computer-generated values as a "baseline," and recording the frequency and degree of deviation from these values recorded on the handwritten records. Data was considered to be "erroneous" if values for $\mathrm{ETCO}_{2}$ varied by more than $20 \%$, and values for $\mathrm{SpO}_{2}$ varied by more than $5 \%$. Due to the lack of well defined ranges for specific patient variables, data collected from the anesthesia monitor will be compared to the values recalled by the individual practitioners surveyed. Discrepancies will be cited as the variation from the monitor values, with 
values greater than the monitor value recorded as a positive number, and values below the monitor value as a negative number.

Following establishment of face validity for this instrument, after review a panel of experts, the research plan was submitted for approval by the investigational review board (IRB) for Virginia Commonwealth University (VCU). Following IRB approval, this instrument was administered to anesthesia providers participating in the actual delivery of anesthesia, and required the provider to recall various aspects of the case from the previous 30 minutes. Actual patient data was collected to compare to the practitioners' recollections. Practitioners utilizing both AIMS and MERS were evaluated, allowing comparison of any influence of the method of recordkeeping on the accuracy of practitioners' recall.

\section{Research Design}

A Solomon four group design was selected to minimize the threat of testing on the outcome of accuracy of recall. Each of the four facilities was be randomly assigned to one of the four groups of this design, a priori, as indicated in Table 2. Sites were matched based upon the attribute of method of anesthesia recordkeeping. Two sites utilized MERS, and two sites used an AIMS. All sites were selected for uniformity of number and experience of anesthesia providers, complexity of anesthetic cases, as well as volume of anesthetic procedures. Two sites received surveys as pre-tests, two sites received education with a trigger film (treatment), and all sites were surveyed 2 months following the time of this presentation and again after a one month delay, as post-tests. 
Data collection took place over as few days as possible to reach a maximum sample size, while limiting the threat of maturation to the group (Issac \& Michael, 1995).

\section{Effect size}

The effect size for the evaluation of the method of recordkeeping has not been well established in the literature. Thrush (1992) cited only frequency distributions, using Fisher's exact test to establish statistical significance. An observation study, the total sample for this study was 13 cases. Total numbers of out-of-range values as compared with total number of recorded values, producing a percentage of out-of-range values were presented, along with $p$ values determined by Fisher's exact test. Effect size cannot be determined from the values provided by the authors of this study.

Weinger et al (1997) provided tables of values indicating the mean number of tasks per minute, along with standard errors for the means, for both manual recordkeeping and electronic recordkeeping. A calculation of Cohen's $d$ indicates an overall effect size of 0.26 for all 26 categories, and an effect size of 0.48 for recordkeeping tasks. Block, Jr. et al, (1991) listed the values of a total of 4,942 blood pressure readings from 118 cases in a retrospective analysis. Values were given only in the form of six graphic charts indicating the percentage of values over ranges of blood pressure values in $10 \mathrm{mmHg}$ increments. The authors indicated that, "Variations in blood pressure and heart rate, common in daily life, appear to be frequent also during anesthesia" (F. E. Block Jr., 1991). The authors also cited previous works of Cook (1989) and Lerou (1988) to indicate a large difference in vital sign values recorded by automatic recordkeeping systems and those recorded 
manually, indicating a phenomenon the authors describe as "smoothing" (F. E. Block Jr., 1991).

Cook (1989) studied differences between handwritten and automatic blood pressure records.(Cook et al., 1989) Effect size was not discussed, and precise values were not indicated by the authors, precluding calculation of Cohen's $d$ from this study. The authors provided scatterplots of maximum and minimum systolic pressure. These graphs displayed one point per case, with a diagonal line indicating the point where electronically and manually recorded values were equal. Of the values recorded for maximum systolic blood pressure, only two fell on this line. Two values fell below this line of equality. All the remaining points (42) fell above the line, indicating that the electronically blood pressure exceeded blood pressures recorded manually. Similar findings were indicated on the scatterplot for minimum systolic blood pressure, with 32 cases falling below the diagonal line of equality, indicating that the electronically recorded blood pressure was lower than blood pressures recorded manually. Results of this study indicated a large effect size for accuracy of electronic records when compared to manually recorded blood pressure values.

Lerou et al (1988) compared automated and handwritten anesthesia records over eight variables: tidal volume, respiratory rate, End-tidal carbon dioxide fraction, inspired oxygen fraction, oxygen saturation via pulse oximetry, heart rate, and systolic and diastolic blood pressure. The authors did not provide precise values for their results, precluding calculation of Cohen's $d$. The authors calculated "error fractions" to indicate the number of minutes of missing data out of a total of 2,412 minutes. The authors found 
smaller values for "error fractions" for the variables of blood pressure and heart rate, yet found a much higher incidence of erroneous or incorrect values between the manually and electronically recorded values. The authors stated that " $57 \%$ more erroneous data are observed for the diastolic blood pressure than for the systolic blood pressure."(Lerou et al., 1988) Subdividing the cases into the time periods of induction, maintenance, and emergence, the authors indicate an error fraction of 0.67 for oxygen saturation and 0.76 for End-tidal carbon dioxidefraction during induction and emergence. Of all the values under study, the authors indicated only two instances of erroneous data recording by the electronic recordkeeping system. While the lack of precise values precluded calculation of effect size, the authors indicated a moderate to large difference in the two forms of recordkeeping.

Considering the lack of defined effect size and the difficulties in calculation of effect size from the data provided by the authors in published research, an effect size of "moderate" was considered in the determination of sample size in power analysis calculations. Calculations for sample size were conducted with an $\alpha$ value of 0.05 , indicating a $5 \%$ risk of Type I error and power of $0.80(\beta=0.20)$, indicating a $20 \%$ risk of Type II error. Values for "moderate" effect size were 0.30 , and "large" effect size was 0.50. (Polit \& Beck, 2004) Based upon these calculations, sample size was based upon a "moderate" effect size, and determined to be 88 total subjects. To achieve the required sample size determined by the power analysis, 44 subjects must be collected for each form of recordkeeping. Each of these two forms of recordkeeping were subdivided among the four facilities, two using AIMS and two using MERS. To achieve the necessary sample 
size, 22 subjects must be obtained at each of the four sites. Due to the lack of specificity of effect size published in the literature, post hoc power analysis was conducted to determine more accurate values for effect size and reduce the possibility of Type I and Type II error. The proposed statistical analyses of data, MANCOVA and profile analysis are robust to differences in sample sizes in cells. (Tabachnick \& Fidell, 2007)

Demographic data was collected from subjects in the form of age, gender, years of experience as a CRNA, and years of experience with the current method of recordkeeping. This demographic data was used as covariates in the MANCOVA analysis. This data was also analyzed to establish similarities between AIMS and MERS groups. In the event of disparate groups, stratified random sampling will be employed. This method may result in a reduction of sample size, and ultimately, in research power. A post hoc power analysis was conducted, with limitations revealed in the discussion of results.

\section{Inclusion/Exclusion Criteria}

Subjects were limited to CRNA anesthesia staff to limit confounding variables. Each facility provides anesthesia services in a team approach, with one CRNA and one anesthesiologist assigned to each surgical case. Anesthesiologists may be assigned to supervise up to four cases, while each CRNA is assigned to only one case, maintaining constant attendance with the patient. Anesthesiologists are available to provide assistance throughout the case, and are present during times of increased workload, such as induction of anesthesia, emergence from anesthesia, or times of hemodynamic instability. Additional differences in level of education, educational methods, and 
experience with method of recordkeeping precluded the inclusion of anesthesiologists in this research. In an attempt to limit any potential effect of lack of experience with the method of recordkeeping or a lack of experience at the facility, subjects were limited to those practitioners with at least 90 days of experience at each location.

The method of anesthesia was limited to general endotracheal anesthesia to further limit confounding variables. Placement of an endotracheal (ET) tube results in a high level of accuracy of measurement of percentages of exhaled gases and inspiratory and expiratory pressures. Other methods of anesthesia do not provide the accuracy of measurement of $\mathrm{ETCO}_{2}$, and IP that accompanies general endotracheal anesthesia, and were be excluded from this study.

Previous research has indicated discrepancies in the accuracy of recordkeeping during periods of increased workload (Biddle et al., 2001; Lerou et al., 1988; Logas et al., 1987). To minimize this effect, subjects were surveyed only during the maintenance phase of the anesthetic, during times of hemodynamic stability, and only after a period of at least 30 minutes of constant attendance to the patient. A full list of inclusion and exclusion criteria may be found in Table 4.

Table 4: Inclusion and Exclusion Criteria

\begin{tabular}{cc}
\hline Inclusion Criteria & Exclusion Criteria \\
\hline CRNA & Non-CRNA \\
$\geq 90$ days of employment at facility & $<90$ days of employment at facility \\
General endotracheal (ET) anesthesia & Absence of ET tube \\
Maintenance phase of anesthetic & Induction/Emergence phase of anesthetic \\
Hemodynamic stability & Absence of hemodynamic stability \\
$\geq 30$ minutes constant attendance to patient & $<30$ minutes constant attendance to patient \\
\hline
\end{tabular}




\section{Statistical Analysis}

The first research question was:

- Do anesthesia providers using AIMS recordkeeping demonstrate equivalent recall accuracy of specific patient variables, as compared with anesthesia providers using a MERS?

For this question, the method of recordkeeping served as the independent variable (IV). The accuracy of the practitioner's recall over the previous sixty minutes of the patient's vital signs, parameters, and events of the anesthetic case served as dependent variables. Vital signs were defined as the highest and lowest heart rate (HR), and highest and lowest systolic blood pressure (SBP). Vital parameters were defined as the highest and lowest $\mathrm{ETCO}_{2}$, and highest IP. Events of the case were defined as the total fluid volume delivered over the anesthetic case. These eight elements served as dependent variables. These variables were chosen due to the typical fluctuation of the HR, SBP, and $\mathrm{ETCO}_{2}$, as well as the fact that these three values are displayed on the largest monitor on the anesthesia machine. IP does not fluctuate in a negative direction, which would indicate a disconnection of the anesthetic circuit, but may rise in response to a decreasing plane of anesthesia, obstruction, or bronchospasm. IP is also displayed on the smallest monitor screen available to the anesthesia provider, separate from all other values studied. Total fluid volume delivered is a continuous measurement that is not recorded automatically by AIMS, and must be entered manually in both forms of recordkeeping. The age of the anesthesia provider, the years of experience as a CRNA, and the years of experience with the current method of recordkeeping were considered as covariates. 
A MANCOVA was performed to determine which DVs are affected by the method of recordkeeping. MANCOVA reduces the risk of Type I error when compared to the option of performing multiple ANCOVA tests (Tabachnick \& Fidell, 2007). The test statistic will be the F statistic, utilizing Pillai's trace, the most robust test for problems with assumptions, such as differences in sample sizes in cells, as will be the case with different numbers of practitioners at the four sites. If the overall $F$-statistic is significant, then univariate tests of group differences will be measured, as well as Roy-Bargmann step-down tests, with the DVs entered in an order defined by the different attributes of the displayed values (HR, SBP, and $\mathrm{ETCO}_{2}$ have a different digital display than IP, which could result in variance due to a difference in presentation). Covariates were entered as DVs, with statistically significant differences noted. Statistically significant correlations between CVs and DVs were compared for the percentage of variance explained by the CVs.

The second research question was:

- Does the instruction of the benefits and limitations of recordkeeping practices by trigger film influence the recall accuracy of specific patient variables by anesthesia providers using AIMS or MERS?

The Solomon four-group design was selected in order to address this research question as shown in Table 2. This form of experimental design assesses the potential of pretest sensitization separate from the effect of the treatment, in this case, the presentation of a trigger film. McGahee \& Tingen (2009) illustrate that the interaction of pretest sensitization has the potential to reduce the generalizability of the outcome 
(McGahee, T. W. \& Tingen, M. S., 2009). Selection of this design provides an additional measure of external validity to a design already strong in internal validity (Braver, M.C.W. and Braver, S. L., 1988). A limitation of this design, as stated by Braver \& Braver (1988), "...is the lack of certainty concerning the proper statistical treatment of this rather complicated design." (Braver, M.C.W. and Braver, S. L., 1988). When selecting a statistical test for this design, it should be noted that no single procedure may make use of all six observations simultaneously. The asymmetric design precludes traditional methods of statistical analysis (Campbell, D.T. and Stanley, J. C., 1963). Due to this limitation, the planned profile analysis (MANOVA for repeated measures) is not feasible.

Braver \& Braver (1988) offered a detailed multi-step process to analyze the data, taking into account all six observations and searching for any pretest sensitization along with any treatment effect. The first step in the process seeks evidence of pretest sensitization. This was accomplished through a 2 X 2 factorial ANOVA of the four posttest scores in the design. Two factors were considered, each with two levels: Pretest, with levels of Pretest and No Pretest; and Treatment, with levels of Treatment or No Treatment. The interaction effect would demonstrate evidence of pretest sensitivity if significant, and would be known as Test A. Also, a simple effect for treatment should also be significant in the first row $\left(\mathrm{O}_{2}\right.$ and $\left.\mathrm{O}_{5}\right)$, known as Test $\mathrm{B}$, but not significant in the second row $\left(\mathrm{O}_{5}\right.$ and $\left.\mathrm{O}_{7}\right)$, known as Test $\mathrm{C}$. Non-significant results indicate no presence of a pretest effect. 
To determine a treatment effect, the main effect of the factor, Treatment, should be significant (Test D). If non-significant, additional testing is required, as Test D does not consider the pretest information available for Groups 1 and 2 (St. Mary's hospital and Inova Fairfax hospital, See Table 2). The addition of this absent data will add power to the data analysis, and may be measured by a two-group ANCOVA on the posttest scores, using the pretest scores as CVs (Test E). A significant result for Test $\mathrm{E}$ is evidence of a treatment effect. If non-significant, testing should continue. To maximize power, analysis must be performed on the untested elements of the design. To measure the effect of the pretest on groups with the method of recordkeeping, a two-group ANOVA is preformed on the initial data collected at each site (Test F). A significant result indicates presence of pretest sensitivity or an effect of the method of recordkeeping. Finally, a $2 \mathrm{X}$ 2 Factorial ANOVA is preformed on the Pretest and Posttest scores of Groups 1 and 2, as indicated in Table 2, known as Test G. This final analysis completes the analysis of all data collected and a significant result indicates the influence of pretest sensitivity (Braver, M.C.W. and Braver, S. L., 1988). 


\section{CHAPTER FOUR: RESULTS}

The primary purpose of this research was to analyze the anesthesia practitioners' accuracy in recalling patient data when using handwritten or manual entry recordkeeping systems (MERS) or a computerized record generated by an Anesthesia Information Management System (AIMS). A second purpose was to determine the effectiveness of a trigger film that detailed benefits and limitations of both methods of recordkeeping. The project sought to answer two research questions:

- Do anesthesia providers using AIMS recordkeeping demonstrate equivalent recall accuracy of specific patient variables, as compared with anesthesia providers using a MERS?

- Does the presentation of the benefits and limitations of recordkeeping practices by trigger film influence the recall accuracy of specific patient variables by anesthesia providers using AIMS or MERS?

To answer these research questions, the following hypotheses were analyzed:

$\mathrm{H}_{1} \quad$ For each anesthetic case, there will be no difference in the accuracy of recall of specific patient variables recorded by an anesthesia provider using AIMS or an anesthesia provider using a MERS.

$\mathrm{H}_{2} \quad$ For each anesthetic case, there will be no difference in the accuracy of recall of specific patient variables recorded by an anesthesia provider who has attended a trigger film presentation on the benefits and limitations of 
recordkeeping methods than a provider that has not received such instruction.

\section{Power Analysis}

Prior to beginning data collection, a power analysis was performed to determine an appropriate sample size. Initial analysis was conducted through the use of charts available in Polit \& Beck (2004). Subsequent analysis was conducted using a stand-alone computer statistical program based upon the work of Cohen (1988), G*Power3.1. This software program is distributed without charge via the internet. G*Power3.1 was designed to provide $a$ priori estimations of statistical power with a known power, $\alpha$ value, and sample size, as well as post hoc estimations of achieved power when $\alpha$ value, sample size, and effect size are known. Estimations of sample size may also be generated, with a known $\alpha$ value, power, and effect size (Faul, Erdfelder, Buchner, \& Lang, 2009).

Based upon the lack of a theoretically, well-defined effect size and the inability to discern an effect size from the published research, a "moderate" effect size (0.3) was chosen for a priori calculations of power analysis and estimations of effect size. These calculations were conducted with an $\alpha$ value of .05, indicating no greater than a $5 \%$ risk of Type I error, and a power of $.80(\beta=0.2)$, indicating no greater than a $20 \%$ risk of Type II error. Sample size was determined to be 88 subjects by charts contained within Polit \& Beck (2004). Entering this data into G*Power3.1, the statistical test selected for Research Question \#1 was MANOVA: Repeated measures, within-between interaction. Effect size was set at $0.3, \alpha$ error probability set at .05 , Power (1- $\beta)$ set at .95 , with 2 
groups (AIMS vs. MERS), and 3 measurements. With these settings, G*Power3.1 indicated a total required sample size of 175 subjects. For Research Question \#2, the statistical test selected was ANOVA: Repeated measures, within-between interaction. Effect size was set at $0.3, \alpha$ error probability set at .05 , Power $(1-\beta)$ set at .95 , with 4 groups (St. Mary's, Inova Fairfax, VCUMC, and Mary Washington), 2 measurements (Pretest - Posttest) and correlation among repeated measures set to 0 . With these settings, G*Power3.1 indicated a total sample size of 106 subjects.

\section{Population and Sampling}

A convenience sample was drawn from a population of 201 CRNAs employed by 4 facilities in Central and Northern Virginia (See Table 1). All subjects were randomly selected on each day of data collection, based upon their assignment to patients and anesthetic cases that fit inclusion/exclusion criteria determined a priori by the researcher and a panel of experts (See Table 4). The researcher did not influence the assignment of CRNAs to cases. All subjects were given a brief overview of the study, and allowed to decline participation. Data collection was conducted as efficiently as possible, in order to capture the maximum possible sample size of practitioners while limiting the threat of maturation to the group (Issac \& Michael, 1995). Completion of the survey instrument was anonymous, with no identifying marks or variables collected from either the subject or the patient. Total sample size after all three repeated measurements were complete was 214 subjects, in excess of the recommended sample size of 175 subjects for Research Question \#1. Total sample size for the Pretest and Posttest groups for Research Question 
\#2 was 127 subjects, which exceeded the recommended total sample size of 106 subjects.

A detailed description of number of subjects by site may be found in Table 5 .

Table 5: Sample Size by Observation and Site

\begin{tabular}{cccccccc}
\hline Group & Survey & Subjects & $\begin{array}{c}\text { Survey I } \\
\text { Post - } \\
\text { treatment }\end{array}$ & Subjects & $\begin{array}{c}\text { Survey II } \\
\text { Post - } \\
\text { treatment }\end{array}$ & Subjects & $\begin{array}{c}\text { Total } \\
\text { Subjects } \\
\text { by Site }\end{array}$ \\
\hline $\begin{array}{c}\text { St. Mary's } \\
\text { (MERS) } \\
\text { Inova }\end{array}$ & $\mathrm{O}_{1}$ & 21 & $\mathrm{O}_{2}$ & 21 & $\mathrm{O}_{3}$ & 22 & 64 \\
$\begin{array}{c}\text { Fairfax } \\
\text { (AIMS) }\end{array}$ & $\mathrm{O}_{4}$ & 22 & $\mathrm{O}_{5}$ & 20 & $\mathrm{O}_{6}$ & 22 & 64 \\
$\begin{array}{c}\text { VCUMC } \\
\text { (AIMS) }\end{array}$ & & & $\mathrm{O}_{7}$ & 21 & $\mathrm{O}_{8}$ & 21 & 42 \\
$\begin{array}{c}\text { Mary } \\
\text { Washington } \\
\text { (MERS) }\end{array}$ & & & $\mathrm{O}_{9}$ & 22 & $\mathrm{O}_{10}$ & 22 & 44 \\
& & & & & & Total & 214 \\
\hline
\end{tabular}

Instrument

In order to assess the research questions and hypotheses, an instrument was developed to evaluate the accuracy of anesthesia providers' recall when recordkeeping with AIMS or MERS. Based upon prior research (Allard et al., 1995; Yablok, 1990), a total of 10 questions were compiled. These questions asked practitioners to recall specific values for the highest and lowest heart rate (HHR, LHR); highest and lowest systolic blood pressure (HSBP, LSBP); highest and lowest end-tidal carbon dioxide $\left(\mathrm{HETCO}_{2}\right.$ and $\left.\mathrm{LETCO}_{2}\right)$; highest and lowest inspiratory pressure (HIP and LIP), the lowest oxygen saturation $\left(\mathrm{LSPO}_{2}\right)$; and the total fluid volume (TFV) administered for the case. With the exception of the TFV administered, all values were to be recalled from the previous 30 minutes. A panel of experts reviewed this instrument and the justifications 
for each variable, and recommended the addition of $\mathrm{LSPO}_{2}$. Unanimous approval was obtained, establishing face validity.

\section{Data Collection}

After review and approval of the project by the panel of experts, application to the Virginia Commonwealth University (VCU) Investigational Review Board (IRB) was completed. The IRB granted an exempt review and a waiver of informed consent, effective December 2009. Written consent was obtained from each of the four facilities participating in the research. One site, Medicorp Mary Washington Hospital, requested an additional application to the facility's internal IRB, and such application was completed and also resulted in an exempt review with waiver of informed consent.

A Solomon four-group research design was implemented, in order to control for the possibility of a pretest effect on the outcome of the trigger film presentation (treatment). Time intervals between interactions at the facilities were kept similar, for homogeneity of sampling. All sites were visited, data collection completed, and treatments administered according to this design, as indicated in Table 6.

Table 6: Data Collection and Treatment Schedule

\begin{tabular}{cccccccc}
\hline Group & Survey & $\begin{array}{c}\text { Time } \\
\text { Interval }\end{array}$ & $\begin{array}{c}\text { Trigger } \\
\text { Film }\end{array}$ & $\begin{array}{c}\text { Time } \\
\text { Interval }\end{array}$ & $\begin{array}{c}\text { Survey I } \\
\text { Post }- \\
\text { treatment }\end{array}$ & $\begin{array}{c}\text { Time } \\
\text { Interval }\end{array}$ & $\begin{array}{c}\text { Survey II } \\
\text { Post - } \\
\text { treatment }\end{array}$ \\
\hline $\begin{array}{c}\text { St. Mary's } \\
\text { (MERS) }\end{array}$ & $\mathrm{O}_{1}$ & 84 days & $\mathrm{X}$ & 62 days & $\mathrm{O}_{2}$ & 28 days & $\mathrm{O}_{3}$ \\
$\begin{array}{c}\text { Inova Fairfax } \\
\text { (AIMS) }\end{array}$ & $\mathrm{O}_{4}$ & 83 days & & & $\mathrm{O}_{5}$ & 35 days & $\mathrm{O}_{6}$ \\
$\begin{array}{c}\text { VCUMC (AIMS) } \\
\begin{array}{c}\text { Mary Washington } \\
\text { (MERS) }\end{array}\end{array}$ & & & $\mathrm{X}$ & 62 days & $\mathrm{O}_{7}$ & 34 days & $\mathrm{O}_{8}$ \\
\hline
\end{tabular}


To collect the data, a trained observer, a qualified anesthesia provider, approached each Certified Registered Nurse Anesthetist (CRNA), gave a brief description of the project, instructions for completion of the instrument, and requested a verbal consent. Once consent was obtained, the subject CRNA was asked to briefly turn away from the monitors to complete the instrument with values from memory. Both the subject and the trained observer remained in constant attendance with the patient. The trained observer accessed the "trends" graphic display of the anesthesia monitoring system, and values for each variable were recorded based upon trended data from the previous 30 minutes, displayed in five minute intervals. Through the series of repeated measurements as listed in Table 5, a total of 214 surveys were collected, with 108 surveys from practitioners utilizing MERS, and 106 from practitioners utilizing AIMS.

\section{Homogeneity of Facilities}

Demographic data was collected for each subject, to serve as CVs in the analysis of data as well as to establish homogeneity of the samples collected from each of the four sites. Four CVs were collected from each subject; age, gender, years of experience, and years of experience with the current method of recordkeeping. An additional variable, ASA physical status classification, was collected to assess the homogeneity of anesthetic case assignments among subjects. A detailed list of the frequency and percentage of CVs may be found in Table 7. In order to identify correlations between the CVs and the method of recordkeeping, Spearman's correlation coefficient was calculated. Spearman's correlation coefficient is "a standard measure of the strength of relationship between two variables that does not rely on the assumptions of a parametric test (Field, 2005), pg. 
745." The outcome of Spearman's correlation coefficient indicated that the covariate "Gender" had a small positive correlation, indicating that the female gender had a small association with AIMS recordkeeping (i.e. Female subjects were more likely to use AIMS recordkeeping). For the covariate "Age," there was a slight negative correlation; this correlation indicated that with increasing age, there was a slight association with MERS recordkeeping. For the covariate "Years of Experience," there was a small negative correlation; this indicates that with increasing years of experience, there was a small association with MERS recordkeeping. The covariate "Length of Employment," had a slight negative correlation, indicating that with increasing length of employment there was a slight association with MERS recordkeeping. The covariate of "Years of Experience with Method of Recordkeeping" had a moderate negative correlation, indicating that with increasing years of experience with a particular method of recordkeeping there was a slight association with MERS recordkeeping. Finally, the covariate of "ASA classification" revealed an extremely slight positive correlation, indicating that increasing ASA classification has a very slight association with AIMS recordkeeping. A detailed account of the significance and correlation of $\mathrm{CV}$ s may be found in Appendix B.

\section{Data Analysis - Research Question 1}

For the first research question, data was analyzed with a Multivariate Analysis of Covariance, or MANCOVA, to determine which (if any) DVs were affected by the method of recordkeeping. This research question and hypothesis is cited below: 
Table 7: Frequency and Percentage of Covariates

\begin{tabular}{|c|c|c|}
\hline Covariate & Frequency & Percent \\
\hline \multicolumn{3}{|l|}{ Gender } \\
\hline Male & 63 & $29.4 \%$ \\
\hline Female & 151 & $70.6 \%$ \\
\hline \multicolumn{3}{|l|}{ Age } \\
\hline$<25$ years & 0 & $0 \%$ \\
\hline $26-35$ years & 65 & $30.4 \%$ \\
\hline $36-45$ years & 72 & $33.6 \%$ \\
\hline $46-55$ years & 56 & $26.2 \%$ \\
\hline$>55$ years & 21 & $9.8 \%$ \\
\hline \multicolumn{3}{|l|}{ Years of Experience } \\
\hline$<5$ years & 79 & $36.9 \%$ \\
\hline $5-10$ years & 54 & $25.2 \%$ \\
\hline $11-15$ years & 15 & $7.0 \%$ \\
\hline $16-20$ years & 28 & $13.1 \%$ \\
\hline$>20$ years & 38 & $17.8 \%$ \\
\hline \multicolumn{3}{|l|}{ Length of Employment } \\
\hline $3-6$ months & 23 & $10.7 \%$ \\
\hline 6 months - 1 year & 24 & $11.2 \%$ \\
\hline $2-5$ years & 81 & $37.9 \%$ \\
\hline $5-10$ years & 41 & $19.2 \%$ \\
\hline$>10$ years & 45 & $21 \%$ \\
\hline \multicolumn{3}{|c|}{ Years of Experience with Method of Recordkeeping } \\
\hline $3-6$ months & 16 & $7.5 \%$ \\
\hline 6 months - 1 year & 14 & $6.5 \%$ \\
\hline $2-5$ years & 72 & $33.6 \%$ \\
\hline $5-10$ years & 47 & $22.0 \%$ \\
\hline$>10$ years & 65 & $30.4 \%$ \\
\hline \multicolumn{3}{|l|}{ ASA Classification } \\
\hline 1 & 17 & $7.9 \%$ \\
\hline 2 & 128 & $59.8 \%$ \\
\hline 3 & 66 & $30.8 \%$ \\
\hline 4 & 3 & $1.4 \%$ \\
\hline 5 & 0 & $0.0 \%$ \\
\hline
\end{tabular}

- Research Question 1: Do anesthesia providers using AIMS recordkeeping demonstrate equivalent recall accuracy of specific patient variables, as compared with anesthesia providers using a MERS? 
$\mathrm{H}_{1} \quad$ For each anesthetic case, there will be no difference in the accuracy of recall of specific patient variables recorded by an anesthesia provider using AIMS or an anesthesia provider using a MERS.

Field (2008) recommended calculation of the F statistic, utilizing Pillai's trace, as the most robust test for problems with assumptions. If the overall F-statistic is significant, then univariate tests of group differences will be measured, as well as RoyBargmann step-down tests, with the DVs entered in an order defined by the different attributes of the displayed values (HR, SBP, and $\mathrm{ETCO}_{2}$ have a different digital display than IP, which could result in variance due to a difference in display). CVs will be entered as DVs in step-down tests, with statistically significant differences noted. Statistically significant correlations between CVs and DVs will be compared for the percentage of variance explained by the CVs (Field, 2005).

\section{Assumptions of MANCOVA}

In order to conduct an analysis that is reliable, certain assumptions must be met before data is analyzed with a particular statistical test (Field, 2005). For most parametric tests, four assumptions must be met: 1) the data must have a normal distribution, 2) there must be homogeneity of variance of the data, 3) the data must be measured on a continuous level, and 4) data from different subjects is independent (Field, 2005). For a multivariate test, such as MANCOVA, both multivariate normality and multivariate homogeneity of variance must also be met(Field, 2005). These assumptions will be discussed individually. 


\section{Independence}

A convenience sample was taken at each of the four facilities, taken from the population of CRNAs assigned to cases in the operating rooms of each of the four facilities of the days of testing. Each practitioner was assigned to rooms and cases without interference from the researcher. Based upon inclusion and exclusion criteria cited in Table 4, each practitioner had an equivalent chance of being selected to serve as a subject in this research. Responses of each individual were completely independent of one another, as the responses of one subject were based solely on their recollection of their patient's variables and were not influenced by the recollections of another subject.

\section{Level and measurement of variables}

To satisfy the assumptions of MANCOVA, the independent variable (IV) must be categorical in nature. In this case, the IV, "Method of Recordkeeping," is categorical practitioners surveyed utilized either "AIMS" or "MERS" recordkeeping. The dependent variables (DVs) in MANCOVA must be continuous. In this study, all DVs were measured on a continuous scale. Each practitioner's response was compared to actual data taken from the anesthesia monitor, and entered as a reflection of the difference recollections that were less than the actual value were entered as negative numbers, while recollections that exceeded the actual value were entered as positive numbers. MANCOVA also assumes homogeneity of the covariates (CVs). Homogeneity of CVs was determined using Levene's test. Levene's test "tests the hypothesis that the variances in different groups are equal (i.e. the difference between the variances is zero). A significant result indicates that variances are significantly different - therefore, the 
assumption of homogeneity of variances has been violated (Field, 2005), page 736. If Levene's test is non-significant, then the variances between the groups are roughly equal. The covariate "Gender" was found to be significant, $(p=.000)$. Analysis of the frequency of respondents, as indicated in Table 7 , reveals that 151 of the 214 subjects $(70.6 \%)$ were female. This data cannot be adjusted, as subjects must be either male or female.

Homogeneity of variance was established for each of the remaining CVs. A complete list of Levene's test of homogeneity of variance may be found in Table 8.

Table 8: Levene's test of Homogeneity of Variance for Covariates

\begin{tabular}{lcc}
\hline \multicolumn{1}{c}{$\begin{array}{c}\text { Covariate } \\
\text { Based on Mean }\end{array}$} & Levene Statistic & Significance \\
\hline Gender & 38.024 & .000 \\
Age & .756 & .386 \\
Years of Experience & .077 & .781 \\
Length of Employment & .005 & .942 \\
Years of Experience with Method of & .029 & .865 \\
Recordkeeping & .516 & .473 \\
ASA Classification &
\end{tabular}

A second test, the Pillai-Bartlett trace, (known as Pillai's trace) was preformed to confirm homogeneity of the CVs (Field, 2005). Pillai's trace is "the sum of the proportion of explained variance on the discriminant function variates of the data" (Field, 2005), pg. 741, and is the most robust test to violations of assumptions for multivariate analysis when sample sizes are equal. Pillai's trace was non-significant for all of the CVs measured by this, indicating that the assumption of homogeneity of variance is valid. In 
addition, Pillai's trace was performed on the variable "Site" to determine homogeneity of variance among all four sites participating in this research. The outcome of this variable confirms that all four sites display homogeneity of variance. A complete list of the outcome of Pillai's trace of the CVs may be found in Table 9.

Table 9: Pillai's Trace - Homogeneity of Variance of Covariates and Site

\begin{tabular}{ccccccc}
\hline Effect & Value & $\mathrm{F}$ & $\begin{array}{c}\text { Hypothesis } \\
\mathrm{df}\end{array}$ & Error df & Significance & $\begin{array}{c}\text { Partial Eta } \\
\text { Squared }\end{array}$ \\
\hline $\begin{array}{c}\text { Gender } \\
\text { Age }\end{array}$ & .68 & 1.1413 & 10.000 & 195.000 & .177 & .068 \\
$\begin{array}{c}\text { Years of } \\
\text { Experience }\end{array}$ & .55 & .1 .144 & 10.000 & 195.000 & .332 & .055 \\
$\begin{array}{c}\text { Length of } \\
\text { Employment }\end{array}$ & .042 & .854 & 10.000 & 195.000 & .577 & .042 \\
$\begin{array}{c}\text { Years of } \\
\text { Experience with } \\
\begin{array}{c}\text { Method of } \\
\text { Recordkeeping }\end{array}\end{array}$ & .034 & .695 & 10.000 & 195.000 & .728 & .034 \\
$\begin{array}{c}\text { ASA Classification } \\
\text { Site }\end{array}$ & .053 & 1.096 & 10.000 & 195.000 & .367 & .053 \\
\hline
\end{tabular}

Linearity of Dependent Variables

Another assumption of MANCOVA is that the DVs have a normal, linear distribution. Satisfaction of this assumption may also be determined though the use of Levene's test. None of the DVs are significant for violation of homogeneity of variance. A detailed list of these values may also be found in Table 9. In addition, histograms of each CV and DV may be found in Appendix C. 


\section{Multivariate Normality}

The assumption of multivariate normality of the DVs cannot be tested through the statistical analysis program SPSS. In order to establish multivariate normality, univariate normality must be established for each DV. Univariate normality is determined using Levene's test (Table 10). Results of this test indicated the satisfactory assumption of multivariate normality for all DVs.

Table 10: Levene's Test of Homogeneity of Variance of Dependent Variables

\begin{tabular}{ccc}
\hline $\begin{array}{c}\text { Dependent Variable } \\
\text { Based on Mean }\end{array}$ & Levene Statistic & Significance \\
\hline High Heart Rate & .718 & .398 \\
Low Heart Rate & .342 & .559 \\
High Systolic Blood Pressure & 1.809 & .180 \\
Low Systolic Blood Pressure & 2.561 & .111 \\
High ETCO 2 & .029 & .864 \\
Low ETCO 2 & 1.568 & .212 \\
Low SPO & \\
High Inspiratory Pressure & .130 & .719 \\
Low Inspiratory Pressure & 1.077 & .301 \\
Total Fluid Volume & .015 & .903 \\
\hline
\end{tabular}

\section{Pairwise Comparisons}

Pairwise comparisons were made between the two forms of recordkeeping, AIMS vs MERS for each of the 10 DVs, using the Bonferroni correction. The Bonferroni correction is "a correction applied to the $\alpha$-level to control the overall Type I error rate when multiple significance tests are carried out (Field, 2005), pg. 725." The outcome of 
this test indicated non-significant results for all DVs, indicating no significant difference between the accuracy of recall based upon the method of recordkeeping. Results of pairwise comparisons may be found in Table 11 .

Table 11: Pairwise Comparisons - Dependent Variables by Method of Recordkeeping

\begin{tabular}{lccccc}
\hline \multicolumn{1}{c}{ Dependent Variable } & \multicolumn{2}{c}{ Method of Recordkeeping } & $\begin{array}{c}\text { Mean } \\
\text { Difference }\end{array}$ & $\begin{array}{c}\text { Standard } \\
\text { Error }\end{array}$ & Significance \\
\hline High Heart Rate & MERS & AIMS & -3.162 & 1.930 & .103 \\
Low Heart Rate & MERS & AIMS & -1.712 & 1.264 & .177 \\
High Systolic Blood Pressure & MERS & AIMS & -1.252 & 2.909 & .667 \\
Low Systolic Blood Pressure & MERS & AIMS & 3.682 & 3.251 & .259 \\
High ETCO & MERS & AIMS & -.915 & .713 & .201 \\
Low ETCO 2 & MERS & AIMS & -1.032 & .614 & .094 \\
Low SPO & MERS & AIMS & -.460 & .604 & .448 \\
High Inspiratory Pressure & MERS & AIMS & .981 & .722 & .176 \\
Low Inspiratory Pressure & MERS & AIMS & -.895 & .743 & .230 \\
Total Fluid Volume & MERS & AIMS & 48.581 & 38.536 & .209 \\
\hline
\end{tabular}

Further data analysis was conducted through the use of Pillai's trace, known to be the most robust measure to violations of assumptions for multivariate analyses when sample size is equal (Field, 2005). The results of Pillai's trace indicated a significance of $p=.116$, or a non-significant result in the comparison of accuracy of measurement between the two forms of recordkeeping (Table 12). 
Table 12: Pillai's Trace - Multivariate tests of method of recordkeeping

\begin{tabular}{cccccccc}
\hline & Value & $F$ & $\begin{array}{c}\text { Hypothesis } \\
\text { df }\end{array}$ & Error df & Significance & $\begin{array}{c}\text { Partial } \\
\text { Eta } \\
\text { Squared }\end{array}$ & $\begin{array}{c}\text { Observed } \\
\text { Power }\end{array}$ \\
\hline $\begin{array}{c}\text { Pillai's } \\
\text { Trace }\end{array}$ & 0.075 & $1.577^{\mathrm{a}}$ & 10.000 & 195.000 & 0.116 & 0.075 & 0.762 \\
\hline
\end{tabular}

Individual univariate tests were also performed on each of the DVs, and are found in Table 13. None of the univariate tests were significant, adding further credibility to the claim that there is no difference in the accuracy of recall among practitioners based upon the method of recordkeeping. As none of the tests were significant, step-down tests were not required and were not conducted.

Table 13: Univariate Tests of Dependent Variables

\begin{tabular}{|c|c|c|c|c|c|c|c|c|}
\hline Dependent Variable & & $\begin{array}{l}\text { Sum of } \\
\text { Squares }\end{array}$ & df & Mean Square & $\mathrm{F}$ & Sig. & $\begin{array}{c}\text { Partial Eta } \\
\text { Squared }\end{array}$ & $\begin{array}{c}\text { Observed } \\
\text { Power }^{\mathrm{a}}\end{array}$ \\
\hline HighHeartRate & Contrast & 390.647 & 1 & 390.647 & 2.683 & .103 & .013 & .371 \\
\hline LowHeartRate & Contrast & 114.613 & 1 & 114.613 & 1.835 & .177 & .009 & .271 \\
\hline HighSBP & Contrast & 61.276 & 1 & 61.276 & .185 & .667 & .001 & .071 \\
\hline LowSBP & Contrast & 529.939 & 1 & 529.939 & 1.283 & .259 & .006 & .204 \\
\hline HighETCO2 & Contrast & 32.704 & 1 & 32.704 & 1.648 & .201 & .008 & .248 \\
\hline LowETCO2 & Contrast & 41.588 & 1 & 41.588 & 2.826 & .094 & .014 & .387 \\
\hline LowSPO2 & Contrast & 8.254 & 1 & 8.254 & .579 & .448 & .003 & .118 \\
\hline HighInspPress & Contrast & 37.612 & 1 & 37.612 & 1.844 & .176 & .009 & .272 \\
\hline LowInspPress & Contrast & 31.282 & 1 & 31.282 & 1.451 & .230 & .007 & .224 \\
\hline TotalFluidVol & Contrast & 92238.088 & 1 & 92238.088 & 1.589 & .209 & .008 & .241 \\
\hline
\end{tabular}

The F tests the effect of Method of Recordkeeping. This test is based upon the linearly independent pairwise comparisons among the estimated marginal means. 


\section{Data Analysis - Research Question 2}

The second purpose of this research was to determine the effectiveness of a trigger film detailing the benefits and limitations of both methods of recordkeeping, through the following research question and hypothesis:

- Does the presentation of the benefits and limitations of recordkeeping practices by trigger film influence the recall accuracy of specific patient variables by anesthesia providers using AIMS or MERS?

$\mathrm{H}_{2}$ For each anesthetic case, there will be no difference in the accuracy of recall of specific patient variables recorded by an anesthesia provider who has attended a trigger film

The Solomon four-group design was selected in order to address this research question (See Table 2). This form of experimental design assesses the potential of pretest sensitization separate from the effect of the treatment, in this case, the presentation of a trigger film. McGahee \& Tingen (2009) illustrate that the interaction of pretest sensitization has the potential to reduce the generalizability of the outcome (McGahee, $\mathrm{T}$. W. \& Tingen, M. S., 2009). Selection of this design provides an additional measure of external validity to a design already strong in internal validity (Braver, M.C.W. and Braver, S. L., 1988). A limitation of this design, as stated by Braver \& Braver (1988), "...is the lack of certainty concerning the proper statistical treatment of this rather complicated design." (Braver, M.C.W. and Braver, S. L., 1988). When selecting a statistical test for this design, it should be noted that no single procedure may 
make use of all six observations simultaneously. The asymmetric design precludes traditional methods of statistical analysis (Campbell, D.T. and Stanley, J. C., 1963). Due to this limitation, the planned profile analysis (MANOVA for repeated measures) is not feasible.

Braver \& Braver (1988) offers a detailed multi-step process to analyze the data, taking into account all six observations and searching for any pretest sensitization along with any treatment effect. Tables that indicate detailed results of all these steps may be found in Appendix D. The first step in the process is to seek evidence of pretest sensitization. This is accomplished through a $2 \times 2$ factorial ANOVA of the four posttest scores in the design $\left(\mathrm{O}_{2}, \mathrm{O}_{5}, \mathrm{O}_{7}\right.$, and $\left.\mathrm{O}_{9}\right)$, as shown in Table 14.

Table 14: 2 X 2 ANOVA of Posttest scores

\begin{tabular}{cccc}
\hline & & Treatment & \\
& & Yes & No \\
Pretest & Yes & $\mathrm{O}_{2}$ & $\mathrm{O}_{5}$ \\
& $\mathrm{No}$ & $\mathrm{O}_{5}$ & $\mathrm{O}_{7}$ \\
\hline
\end{tabular}

Two factors are considered, each with two levels: Pretest, with levels of Pretest and No Pretest; and Treatment, with levels of Treatment or No Treatment. The interaction effect will demonstrate evidence of pretest sensitivity if significant, and will be known as Test A. Also, a simple effect for treatment should also be significant in the first row $\left(\mathrm{O}_{2}\right.$ and $\left.\mathrm{O}_{5}\right)$, known as Test $\mathrm{B}$, but not significant in the second row $\left(\mathrm{O}_{5}\right.$ and $\left.\mathrm{O}_{7}\right)$, known as Test C. Non-significant results indicate no presence of a pretest effect.

To determine the effect of the treatment, the main effect of the factor should be significant (Test D). If non-significant, additional testing is required, as Test D does not 
consider the pretest information available for Groups 1 and 2 (St. Mary's hospital and Inova Fairfax hospital, See Table 2). The addition of this absent data will add power to the data analysis, and may be measured by a two-group ANCOVA on the posttest scores, using the pretest scores as CVs (Test E). A significant result for Test $\mathrm{E}$ is evidence of a treatment effect. If non-significant, testing should continue. To maximize power, analysis must be performed on the untested elements of the design. To measure the effect of the pretest on groups with the method of recordkeeping, a two-group ANOVA is preformed on the initial data collected at each site (Test F), as shown in Table 15. A significant result indicates presence of pretest sensitivity or an effect of the method of recordkeeping.

Table 15: Two-way ANOVA of Pretest Data by Method of Recordkeeping

\begin{tabular}{ccc}
\hline & \multicolumn{2}{c}{ Data Collection } \\
Method of Recordkeeping & Initial Sample & Initial Sample \\
MERS & $\mathrm{O}_{1}$ & $\mathrm{O}_{9}$ \\
AIMS & $\mathrm{O}_{4}$ & $\mathrm{O}_{7}$ \\
\hline
\end{tabular}

Finally, a 2 X 2 Factorial ANOVA is preformed on the Pretest and Posttest scores of Groups 1 and 2 (See Table 2), known as Test G. This final analysis completes the analysis of all data collected and a significant result indicates the influence of pretest sensitivity (Braver, M.C.W. and Braver, S. L., 1988). Data was analyzed according to the recommendations of Braver \& Braver (1988). Each DV was assessed separately. Results of the tests will be discussed in greater detail, below. Complete details of the full analysis may be found in Appendices D - M. 


\section{Tests A-D - Pretest Sensitivity/ Treatment Effect}

Analyses for all dependent variables in Test A indicated no statistical significance. Test B, measurement of treatment effect in row 1 of the 2 X 2 ANOVA, was also non-significant for all DVs. Test C, determining the effect of pretest sensitivity on the outcome of the treatment, was also non-significant for all groups. Test D, the measurement of the effect of the treatment, was non-significant for all DVs. Results of these tests indicate a low likelihood of pretest sensitivity, but also the absence of any influence of the trigger film on subsequent measures of accuracy.

\section{Further Testing - Tests $E-G$}

As indicated by Braver \& Braver (1988), non-significant results require further testing. Test E revealed a significant result for the DVs, "HSBP", "LSBP", and "TFV". Test E was non-significant for all other DVs.

Of these significant results, the DV, "HSBP," was significant in the interaction effect of the posttest scores and the pretest scores as a covariate. This result, Posttest $\mathrm{x}$ $\mathrm{O} 4$, had a value $p=.028$. This result indicates a significant difference between the pretest and posttest scores for the DV of "HSBP" at the Inova Fairfax hospital site. This site did not receive a treatment (trigger film), so this result may indicate the effect of the pretest on the posttest for this DV.

The DV, "LSBP," was also significant for the interaction effect of posttest scores with pretest scores used as a covariate. This result, Posttest $\mathrm{x} O 1$, had a value of $p=.046$. This result indicates a significant difference between the pretest and posttest scores 
recorded at the St. Mary's hospital site. This site received education with a trigger film, so this result may indicate either an effect of the pretest or the treatment for this DV.

The DV, "TFV," was significant for Test $\mathrm{E}$ with a result of $p=.014$. This result was for the interaction, Posstest x O1, indicating the St. Mary's hospital site, which received the trigger film treatment. This result could indicate an effect of the pretest, or an effect of the treatment on posttest scores for this DV.

Further testing of pretest sensitivity was conducted, through Tests F and G. For Test $\mathrm{F}$, the measure of pretest sensitivity and method of recordkeeping, one DV produced significant results - LSBP. For the DV, "LSBP," results of the interaction, "Pretest $\mathrm{x}$ Method of Recordkeeping" was significant, with $p=.036$. This result indicates that there was a significant difference between the initial scores for accuracy between sites with similar methods of recordkeeping for this DV.

The final test to measure pretest sensitivity, Test G, measured the differences between the pretest and posttest results for the first two groups, St. Mary's hospital and Inova Fairfax hospital. Only St. Mary's hospital received the trigger film treatment. Test G revealed significant results for the DVs, "LSBP" and "LIP." Both of these DVs were significant for the Pretest $\mathrm{x}$ Treatment interaction, with "LSBP" $p=.013$, and "LIP" $p=.020$. This result may indicate the presence of a treatment effect for these DVs. 


\title{
CHAPTER FIVE: CONCLUSIONS
}

\author{
Introduction
}

In the United States, each of the over 50 million anesthetics administered each year generates a detailed record of vital signs, medications, and events of the surgery or procedure (Ishizawa, 2011). Based upon monitoring standards established in 1986, and adopted by both the American Society of Anesthesiologists and the American Association of Nurse Anesthetists, anesthesia providers document oxygenation, ventilation, circulation, and temperature (Eichhorn et al., 1986). Initially developed as a means to improve individual practice, the creation of a record of events and techniques of anesthesia has been in existence since 1894 (Beecher, 1920).

As the science of anesthesia delivery advanced, the demands of documentation on the anesthesia provider increased. Electronic means of recordkeeping was proposed as early as 1929 in an effort to reduce the workload of the anesthetist while preserving the accuracy of the record (McKesson, 1934). Such automation of the record did not take precedence over the handwritten record, and only the development of computing technology in the early 1970's brought about a resurgence of interest in an electronically generated anesthesia chart (Drui et al., 1973).

Throughout the succeeding decades the call for automation of anesthesia documentation increased as computing technology expanded, with claims of 
enhanced time management (Heinrichs et al., 1997), improved data collection for quality improvement (Vigoda et al., 2006), and superior capture of billing elements (Kheterpal et al., 2007). These claims have been met with concerns of potential malpractice liability (Feldman, 2004), significant financial investment (O'Sullivan et al., 2007), increased complexity of tasks (Kennedy, Feingold, Wiener, \& Hosek, 1976) and decreased vigilance related to a decrease in attentiveness to the patient and monitors (Abenstein et al., 1992). Studies analyzing vigilance and workload indicated no significant differences between the two methods (Loeb, 1994; Thrush, 1992; Yablok, 1990). Many of these studies prove to have significant flaws, such as small sample size or methodological errors (Thrush, 1992)(Loeb, 1994), or failure to account for extraneous variables such as the effect of expectancy (Loeb, 1994)(Weinger et al., 1997). In addition, many experts in the field have branded the adoption of anesthesia information management systems (AIMS) as inevitable (Hamilton, 1990; Levitan, 2008; Vigoda et al., 2006).

In 2005, the United States government set a goal to have an electronic medical record for each citizen by 2014, and financial incentives for physicians, medical practices, and hospitals have been included in a recent $\$ 19$ billion economic stimulus package that includes the use of Anesthesia Information Management Systems (AIMS) (Huslin, 2009). Adoption of AIMS has increased, with over $44 \%$ of academic medical centers utilizing or planning to implement such a system in 2008 (Egger Halbeis et al., 2008). Driven by financial incentives, and "a need to mitigate increasing reporting requirements imposed by various regulatory bodies (Ehrenfeld \& Rehman, 2010), Page 
2," widespread adoption of AIMS is occurring without a satisfactory answer to the question of the effect of inattention of practitioners to patient monitoring.

This study sought to improve upon the experiments of the past and examine the effect of a potential treatment to enhance accuracy of memory. In an attempt to explore the attention of anesthesia providers, the accuracy of practitioners' recall of specific vital signs, parameters and events by anesthetists utilizing AIMS and manual entry recordkeeping systems (MERS) was assessed. In addition, the application of an educational program in the form of a trigger film was presented to select groups, to evaluate the effect of the presentation of benefits and limitations of each form of recordkeeping on the accuracy of anesthetists' recall.

\section{Summary of the Study}

Certified registered nurse anesthetists (CRNAs) participate in the administration of more than 30 million of the more than 50 million anesthetics delivered each year (American Association of Nurse Anesthetists, 2008). For each anesthetic administered, a detailed record is generated that includes vital signs, medications, and events of the surgery or procedure. Patients are monitored according to standards published by both the American Society of Anesthesiologists and the American Association of Nurse Anesthetists (AANA). These standards detail the vital signs and parameters that must be recorded by an anesthesia provider to document the monitoring of oxygenation, ventilation, circulation, and temperature (AANA - scope and standards for nurse anesthesia practice. 2007; ASA, 2005). Since the development of the standards, the overwhelming majority of anesthesia providers have recorded this data manually on 
pre-printed forms. This form of manual entry recordkeeping (MERS) persists in more than 90 percent of anesthesia practices (Levitan, 2008). Anesthesia information management systems (AIMS) are reported to be utilized in five to ten percent of hospitals across the United States (Ehrenfeld \& Rehman, 2010). One manufacturer of anesthesia delivery systems and monitors, Drager Medical, states that the Innovian ${ }^{\circledR}$ AIMS is in place in over 130 hospitals across the United States, and is used by more than 7,000 practitioners world-wide (Innovian anesthesia - your competitive advantage. 2008).

\section{Population and Recruitment}

A convenience sample was drawn from a population of over 200 CRNAs employed by 4 facilities in Central and Northern Virginia (See Table 1). All subjects were randomly selected on each day of data collection, based upon their assignment to patients and anesthetic cases that fit inclusion/exclusion criteria determined a priori by the researcher and a panel of experts (See Table 4). The researcher did not influence the assignment of CRNAs to cases. All subjects were given a brief overview of the study, and allowed to decline participation. Data collection was conducted as efficiently as possible, in order to capture the maximum possible sample size of practitioners while limiting the threat of maturation to the group (Issac \& Michael, 1995). Completion of the survey instrument was anonymous, with no identifying marks or variables collected from either the subject or the patient. Total sample size after all three repeated measurements were complete was 214 subjects. 


\section{Instrument}

A standardized instrument was developed (See Appendix 1) based upon previous research (Yablok, 1990; Allard et al, 1995), to evaluate the accuracy of anesthesia providers' recall when recordkeeping with AIMS or MERS. Eight questions were developed, asking practitioners to recall specific data for highest and lowest heart rate (HR), highest and lowest systolic blood pressure (SBP), highest and lowest end-tidal carbon dioxide $\left(\mathrm{ETCO}_{2}\right)$, total fluid volume administered, and highest inspiratory pressure (IP), all within the last thirty minutes of the case. Use of the 30 minute time interval was selected to ensure measurement of recall during part of the maintenance phase of the anesthetic, a documented time of reduced workload (Allard et al., 1995; Weinger et al., 1997; Weinger et al., 2004). A team of experts consisting of a CRNA with a $\mathrm{PhD}$ in epidemiology, an anesthesiologist responsible for the selection and implementation of an AIMS at the VCU Medical Center, a professor and anesthesiologist with expertise in AIMS development and implementation, a nurse practitioner with expertise in the field of information technology, and a professor of cognitive psychology, reviewed the instrument and justifications for the selected questions to establish face validity.

\section{Data Collection}

To collect the data, a trained observer, who was a qualified anesthesia provider, approached each Certified Registered Nurse Anesthetist (CRNA), gave a brief description of the project, instructions for completion of the recall accuracy instrument, and requested a verbal consent. Once consent was obtained, the subject CRNA was 
asked to briefly turn away from the monitors to complete the instrument with values from memory. Both the subject and the trained observer remained in constant attendance with the patient. The trained observer accessed the electronically archived vital signs from the anesthesia monitoring system, and values for each variable were recorded based upon trended data from the previous 30 minutes, displayed in five minute intervals. Through the series of repeated measurements as listed in Table 5, a total of 214 surveys were collected, with 108 surveys from practitioners utilizing MERS, and 106 from practitioners utilizing AIMS.

\section{Overview of the Problem}

The primary purpose of this research was to analyze the anesthesia practitioners' accuracy in recalling patient data when using manual entry recordkeeping systems (MERS) or a computerized record generated by an Anesthesia Information Management System (AIMS). A second purpose was to determine the effectiveness of a trigger film that detailed benefits and limitations of both methods of recordkeeping. The project sought to answer two research questions:

- Do anesthesia providers using AIMS recordkeeping demonstrate equivalent recall accuracy of specific patient variables, as compared with anesthesia providers using a MERS?

- Does the presentation of the benefits and limitations of recordkeeping practices by trigger film influence the recall accuracy of specific patient variables by anesthesia providers using AIMS or MERS?

To answer these research questions, the following hypotheses were analyzed: 
$\mathrm{H}_{1} \quad$ For each anesthetic case, there will be no difference in the accuracy of recall of specific patient variables recorded by an anesthesia provider using AIMS or an anesthesia provider using a MERS.

$\mathrm{H}_{2} \quad$ For each anesthetic case, there will be no difference in the accuracy of recall of specific patient variables recorded by an anesthesia provider who has attended a trigger film presentation on the benefits and limitations of recordkeeping methods than a provider that has not received such instruction.

\section{Review of Methodology}

A Solomon four group design was selected to minimize the threat of testing on the outcome of accuracy of recall. Each of the four facilities was be randomly assigned to one of the four groups of this design, a priori, as indicated in Table 2. Sites were matched based upon the attribute of method of anesthesia recordkeeping. Two sites utilized MERS, and two sites used an AIMS. All sites were selected for uniformity of number and experience of anesthesia providers, complexity of anesthetic cases, as well as volume of anesthetic procedures. Two sites received surveys as pre-tests, two sites received education with a trigger film (treatment), and all sites were surveyed 2 months following the time of this presentation and again after a one month delay, as post-tests. Data collection was conducted over as few days as possible to achieve a maximum sample size, while limiting the threat of maturation to the group (Issac \& Michael, 1995). Completion of the survey instrument was anonymous, with no identifying marks or variables collected from either the subject or the patient. Total sample size after all three 
repeated measurements were complete was 214 subjects, in excess of the recommended sample size of 175 subjects for Research Question \#1. Total sample size for the Pretest and Posttest groups for Research Question \#2 was 127 subjects, which exceeded the recommended total sample size of 106 subjects. The recommended sample sizes cited were obtained by a priori power analysis.

\section{Results}

\section{Research Question \#1}

For the first research question, data was analyzed with a Multivariate Analysis of Covariance, or MANCOVA, to determine which (if any) DVs were affected by the method of recordkeeping. This research question and hypothesis is cited below:

- Research Question 1: Do anesthesia providers using AIMS recordkeeping demonstrate equivalent recall accuracy of specific patient variables, as compared with anesthesia providers using a MERS?

$\mathrm{H}_{1} \quad$ For each anesthetic case, there will be no difference in the accuracy of recall of specific patient variables recorded by an anesthesia provider using AIMS or an anesthesia provider using a MERS.

Assumptions of MANCOVA were met, and covariates were analyzed to establish homogeneity. A criticism of previous studies comparing AIMS and MERS has been a lack of homogeneity of subjects and complexity of anesthetic cases. To address this criticism, analysis of the covariates (Gender, Age, Years of Experience, Length of Employment, Years of Experience with Method of Recordkeeping, and ASA 
Classification) was conducted and found to be homogenous, with the exception of Gender. The covariate of gender was found to be heavily skewed toward the female gender, primarily as a result of subjects participating from the Inova Fairfax site. Of a total of 64 subjects from Inova Fairfax Hospital, only 5 subjects were male. Overall, 63 subjects were male, and 151 were female.

A potential limitation of this research is the collection of data over several different sites. Specific analysis of the variable "Site" was preformed, to establish homogeneity of variance among all four sites participating in this research. The sites were also found to be homogenous. A detailed account of overall homogeneity of variance for covariates and sites may be found in Table 9 .

Data analysis was then conducted between subjects utilizing AIMS recordkeeping and those utilizing MERS over a total of 10 dependent variables (DVs). These dependent variables were: high and low heart rate, high and low systolic blood pressure, high and low end-tidal carbon dioxide levels, low oxygen saturation, high and low inspiratory pressure, and total fluid volume administered. All DVs were determined to have homogeneity of variance (Table 10). Pairwise comparisons between the two groups were found to be non-significant for all DVs (Table 11). An additional test, Pillai's trace, was conducted, and also found to be non-significant (Table 12). Individual univariate tests were conducted on all of the DVs, and were also found to be non-significant. Based upon these findings and the power analysis conducted, the hypothesis, 
$\mathrm{H}_{1}$ For each anesthetic case, there will be no difference in the accuracy of recall of specific patient variables recorded by an anesthesia provider using AIMS or an anesthesia provider using a MERS.

may be accepted with no greater than a $20 \%$ risk of a Type II error.

\section{Research Question \#2}

The second purpose of this research was to determine the effectiveness of a trigger film detailing the benefits and limitations of both methods of recordkeeping, through the following research question and hypothesis:

- Does the presentation of the benefits and limitations of recordkeeping practices by trigger film influence the recall accuracy of specific patient variables by anesthesia providers using AIMS or MERS?

$\mathrm{H}_{2}$ For each anesthetic case, there will be no difference in the accuracy of recall of specific patient variables recorded by an anesthesia provider who has attended a trigger film

The choice of the Solomon four-group design was selected to best answer this research question. Selection of this design allows the researcher to distinguish between the treatment effect and the effect of pretest sensitivity (Braver, M.C.W. and Braver, S. L., 1988). Unless analyzed, the effect of pretest sensitivity has the potential to limit the generalizability of the outcome (McGahee, T. W. \& Tingen, M. S., 2009). A detailed description of this design may be found in Table 2.

Statistical analysis of this design was challenging, as no one single procedure may make use of all six observations simultaneously (Braver, M.C.W. and Braver, S. L., 
1988). Due to this limitation, a MANOVA for repeated measures (profile analysis) is not feasible. Braver and Braver (1988) described a multi-step process of ANOVA measurements to analyze each DV separately. Due to the selection of ANOVA for repeated measurements, the total number of subjects was limited to only those subjects who participated in these six measurements, comprising the pretest and initial posttest groups of this design. Power analysis through $\mathrm{G}^{*}$ Power3.1 indicated a total sample size requirement of 106 subjects for an $\alpha$ value of .05 and a power of .80 . The total sample size for these six measurements was 127 subjects.

Tests $A-D$

Braver and Braver (1988) indicate that the initial analysis of a DV is to assess the influence of pretest sensitization through a 2 X 2 ANOVA of the four posttest scores in the design (Test A). Then, if the treatment has an influence on the outcome, a simple effect for treatment should be significant for the groups who received the treatment, in the first row of this $2 \times 2$ ANOVA (Test B), but not in the second row of the design, which contains the groups that did not receive the treatment (Test $\mathrm{C}$ ). To determine the effect of the treatment, the main effect should be significant (Test D).

Results of tests $a-d$. Analysis of all DVs in Test A indicated no statistical significance. The measurement of the treatment effect, Test B, was also non-significant for all DVs. Test C and D were also non-significant for all DVs. Results of these tests indicate no evidence of pretest sensitivity, but also no evidence of a treatment effect. As indicated by Braver and Braver (1988), testing must continue. 
Tests $E-G$

In the event of a non-significant result for the treatment in Test $\mathrm{D}$, additional testing is required. Test $\mathrm{D}$ does not consider the pretest results of the first two groups (St. Mary's Hospital and Inova Fairfax Hospital, Table 2). Adding this data will add power to the analysis and was measured by a two-group ANCOVA on posttest scores, using pretest scores as covariates (CVs). This test is known as Test E, and results may be found in Appendix D. A significant result for Test E indicates evidence of a treatment effect. If non-significant, testing must continue.

To maximize power, analysis must be performed on the untested elements of the design. To measure the effect of the pretest on groups by method of recordkeeping, a two-group ANOVA was performed on the initial data collected at each site, known as Test F (Table 15). A significant result of this test indicates the presence of pretest sensitivity or an effect of the method of recordkeeping on the results. Test G consists of a 2 X 2 Factorial ANOVA on the pretest and posttest scores of groups 1 and 2 (Table 2), with a significant result indicating the presence of pretest sensitivity.

Results of tests $e-g$. Test E, a measurement of the treatment effect, was significant for the DVs High Systolic Blood Pressure (HSBP), Low Systolic Blood Pressure (LSBP), and Total Fluid Volume (TFV). All remaining DVs were nonsignificant for Test E. The DV HSBP was significant for the interaction effect of the posttest scores using pretest scores as a covariate. This result indicated a significant difference between pretest and posttest scores at the Inova Fairfax Hospital site. As this site did not receive the trigger film educational program, this difference cannot be 
attributed to a treatment effect. The DVs LSBP and TFV were significant for the interaction between the pretest and posttest scores at St. Mary's Hospital, a site that received the trigger film treatment. These results may have indicated evidence of a treatment effect for these two DVs.

Test F, a measure of pretest sensitivity and method of recordkeeping, produced significant results for only a single DV, LSBP. This result indicated a significant difference between the initial scores of accuracy between sites with similar methods of recordkeeping for this DV. Interpretation of this result indicated that there was a significant difference between sites utilizing AIMS recordkeeping and MERS for the DV LSBP.

Test G, the final analysis of the Solomon four-group design, measured differences between the pretest and posttest results for the first two groups, St. Mary's Hospital and Inova Fairfax hospital. This test revealed significant results for the DVs LSBP and Low Inspiratory Pressure (LIP). Both DVs were significant for the interaction between the pretest and the treatment, indicating the potential presence of a treatment effect for LSBP and LIP at St. Mary's Hospital.

Interpretation of this complex analysis indicated that none of the DVs consistently revealed either an effect of pretest sensitization or treatment effect. Following the stepwise analysis proposed by Braver \& Braver (1988), only a single DV, LSBP, indicated significant values consistent with a treatment effect, and these values were significant at only a single site, St. Mary's Hospital. Overall, the lack of consistent significant results for the majority of DVs indicated that the hypothesis 
$\mathrm{H}_{2}$ For each anesthetic case, there will be no difference in the accuracy of recall of specific patient variables recorded by an anesthesia provider who has attended a trigger film

may be accepted, with no greater than a $20 \%$ risk of Type II error.

Relation of Results to the Literature

Many studies have attempted to define the superiority of AIMS or MERS, through the examination of vigilance, accuracy, completeness, enhanced capture of billing elements and cost containment, medico-legal protection of anesthesia practitioners, and quality improvement (Allard et al., 1995; Kay \& Neal, 1986; Loeb, 1994; N. S. Paget et al., 1981a; Thrush, 1992; Weinger et al., 1997; Yablok, 1990; Zollinger et al., 1977). Beyond research, opinions have raised controversy based upon experiences and assumptions that may or may not be based on fact (Lees, 1985; T. N. Noel, 1986; K. R. Noel, 1991; Rosen \& Rosenzweig, 1985a; Saunders, 1990). The area of enhanced legibility of an anesthetic record produced by an AIMS has been well accepted (F. E. Block Jr., 1991; Driscoll et al., 2007; Edsall et al., 1993; Hogan \& Wagner, 1997; Lerou et al., 1988; Logas et al., 1987; Zollinger et al., 1977). The benefits of an AIMS for the purposes of quality improvement and enhanced capture of billing elements have also been well received by the anesthesia community (Benson et al., 2000; Egger Halbeis \& Epstein, 2008; Jost et al., 2003; Lubarsky et al., 1996; Lubarsky et al., 1997; O'Sullivan et al., 2007; A. Petry et al., 1993b; Reich et al., 2005; K. V. Sanborn et al., 1996a). 
Other elements of the anesthetic record have proven to be more controversial. The concepts of accuracy and vigilance have produced strong and conflicting opinions (Lees, 1985; T. N. Noel, 1986; Rosen \& Rosenzweig, 1985a; Rosen \& Rosenzweig, 1985b; Rosen \& Rosenzweig, 1986; Saunders, 1990). The concern of artifact or erroneous data, especially when seen as a potential risk of exposure to medical malpractice suits has been an issue since the first inception of AIMS (Cook et al., 1989; Driscoll et al., 2007; Feldman, 2004; Gage, 1992; Gibbs, 1989a; Hogan \& Wagner, 1997; Lerou et al., 1988; Logas et al., 1987; Shibutani et al., 1990; Vigoda \& Lubarsky, 2006a; Vigoda \& Lubarsky, 2006b; Zollinger et al., 1977). The effect of the method of recordkeeping on vigilance of the anesthesia provider has been studied by multiple authors (N. S. Paget et al., 1981a) (Kay \& Neal, 1986) (Weinger \& Englund, 1990) (Loeb, 1994) (Woods et al., 1995)(Thrush, 1992; Weinger et al., 1997). Paget et al (1982) defined many of the impediments to vigilance in the anesthesia provider. Kay and Neal (1986) suggested that use of automated systems - automated blood pressure devices - may result in a decrease in vigilance in anesthesia providers. Weinger and Englund (1990) called for automation, but with "intelligence," in the form of alarms and visual alerts to call attention to deviation from normative values. Loeb (1995) attempted to directly study vigilance of anesthesia residents through the use of a "vigilance task" and a second anesthesia provider to act as a scribe. This research was criticized by Woods et al (1995), who indicated that a "scribe" was another human anesthesia provider who could participate in the delivery of anesthesia. The authors contended that a computerized recordkeeping system could not participate in anesthesia delivery, either through direct 
action or by approval of the actions of the subject. The author also failed to identify the possibility of an expectancy effect in the conduction of the vigilance task. Weinger et al (1997) also attempted to directly manipulate vigilance through the use of a vigilance task, in this case, a light that would illuminate until a button was pressed. As with Woods et al (1995), no mention was made of the possibility of an expectancy effect by the subjects to the vigilance task. Thrush (1992) examined accuracy of data recorded by anesthesia providers using AIMS and those using MERS. This research claimed definitive results in favor of superior accuracy of practitioners using an AIMS, yet was subject to many methodological flaws. The study population was very small in size (13 cases), and data was collected on widely dissimilar cases, and a disparate number of records were generated by the subjects. Four cases did not record data for the full time allotted, due to the need to emergently initiate cardiopulmonary bypass, indicating a significant difference in workload and task density, yet these cases were included in the results.

In spite of any controversy clouding adoption of AIMS, potential benefits of computerized systems have resulted in the call for increased adoption (Stoelting, 2001). Some authors feel that the widespread adoption of AIMS would be a part of the solution to the unacceptable rate of medical error illustrated by the landmark document, "To Err is Human," published by the Institute of Medicine in 1999 (Kohn et al., 1999). The advancement of computing technology, the effort to standardize medical terminology and electronic records, and the potential cost savings of consolidating all areas of medical care into a single system, all serve to support the claim that AIMS are "poised to revolutionize anesthesia care" (Levitan, 2008). The proponents of computerization 
appear to be overcoming the critics of AIMS, in that $44 \%$ of academic medical centers in the United States have implemented, or are in the process of implementing an AIMS as of 2008(Egger Halbeis et al., 2008). Critics claim some leverage by raising fears of the development of "fully automated anesthesia systems," such as the Canadian "McSleepy" (Shelley, 2008). Despite these fears, however, the United States government has strengthened the call for more advanced technology to be applied to the health care field, and specifically to medical records (Huslin, 2009). In 2005, a goal was set to have an electronic medical record in place for each citizen of the United States by the year 2014 (Huslin, 2009). While obstacles to this goal echo many of the concerns of anesthesia providers regarding AIMS, financial incentives for physicians, medical practices, and hospitals included in the recent $\$ 19$ billion economic stimulus package may serve to further increase adoption of computerized systems, including AIMS (Huslin, 2009).

This research was conducted in response to the controversial claims of superiority of AIMS over MERS, particularly in the area of accuracy and vigilance through attentiveness to monitors and anesthetic record. The study sought to limit extraneous variables by thoughful selection of homogenous clinical facilities, subjects, and ASA classification of cases (See Table 9). Careful analysis was conducted in an attempt to collect an adequate sample size that would enhance research power and enhance generalizability of results. Development of the instrument included detailed justification of all variables and was unanimously approved by a panel of experts prior to implementation. Data analysis revealed an overall lack of significant differences between the recall of practitioners using AIMS recordkeeping and those using MERS. 
In addition to the primary purpose, this research included a secondary goal to examine the effect of a trigger film presentation on the outcome of the accuracy of practitioners' recall of specific patient variables and events. The use of trigger films has a long history in medical education (Ber \& Alroy, 2001), and they have more recently been utilized in the education of CRNAs (W. Hartland et al., 2003). For this research, a trigger film was developed to present the benefits and limitations of each method of recordkeeping, based upon an extensive review of the literature. Once developed, the film was carefully edited by a team of experts, and then presented to two groups of CRNAs as indicated by the research design (See Table 2). Equivalent time elapsed between measurements of all groups following presentation, prior to subsequent data collection at all sites (See Table 6). A complex data analysis was conducted, guided by the literature (Braver, M.C.W. and Braver, S. L., 1988), on the initial pretest and posttest data. Lack of significant evidence of an effect of this treatment was noted, and further analysis of a secondary posttest was not indicated and was not conducted.

The information processing theory includes a widely accepted theory of attention, known as attenuation. In attenuation, stimuli are not entirely blocked from memory, but rather the signals are "turned down," or attenuated to partially filter the vast amount of information presented (Treisman, 1960). This theory is supported by studies of the neurologic system, as noted by Driver (2001). Attention to information depends largely on the context of the situation or the importance of perceived stimuli, yet unconscious attention may still occur (Driver, 2001). In the operating room environment, anesthesia monitors continuously display data on the patient's condition, through both numeric or 
graphic visual displays, accompanied by auditory tones and alarms that alert the provider to extreme values. Operating room personnel give orders, request instruments or information, and may converse with one another. Occasionally, these personnel interact with the anesthesia provider, requesting information about the patient's condition, or to ask for changes in the patient's position or vital signs. The anesthetist perceives all these stimuli, and processes important information, such as trends in vital signs or extreme values that require intervention. Irrelevant or distracting stimuli is disregarded, often without being consciously aware of the level of attention or dismissal given to certain stimuli. Much of the attenuation of this data takes place through top-down processing (Robinson-Riegler \& Robinson-Riegler, 2004), as data presented stimulates previous experiences or expectations that define perception and is then linked to this stored information in memory. It is also proposed that individuals encode large amounts of information into smaller groups, or chunks of data. By categorizing information in this manner, memory of the data is improved. Recall of a ten digit telephone number is often achieved by dividing the number into two chunks, a three digit area code and a 7 digit telephone number. In this manner, we are able to reduce a ten digit number into two numbers of 7 digits or less, aiding our memory (Miller, 1956). Note-taking during lecture is considered a form of chunking (Robinson-Riegler \& Robinson-Riegler, 2004), as would the creation of a handwritten record. The skilled memory theory (Ericsson, K. A. and Staszewski, J. J., 1989) proposes that individuals with significant expertise in a given topic may directly encode information about this topic into long term memory, as a 
skilled anesthetist could encode values for patient information directly into memory when using an AIMS recordkeeping system.

\section{Limitations}

\section{Reliability of the Instrument}

Justification for each of the DVs was presented and reviewed by a panel of experts during the process of establishing face validity of the instrument to be used for data collection. Of these DVs, Inspiratory Pressure (IP) was selected due to the fact that the display of this variable was located in a much smaller and separate screen than other patient variables (Figures $4 \& 5$ ), and values were documented directly onto the anesthesia record in AIMS, and would be recorded manually by practitioners using MERS. Data collection revealed that, unlike the Drager Innovian AIMS utilized by the VCU Medical Center, the Eko Frontiers AIMS utilized by Inova Fairfax Hospital did not automatically record IP, and practitioners were required to manually enter this variable. As a result, the data for these two DVs (High IP and Low IP), were entered manually at three of the four sites, and automatically at only one site. The data collected and analyzed for these two DVs must be considered unreliable, and no conclusions will be made based upon the results of these variables.

\section{Threats to Internal and External Validity}

Data collection at multiple sites is both strength and a limitation of the study. Multiple sites enhance the external validity of the research, but also predispose the study to the threat of selection. Drawing a convenience sample of subjects based upon their availability at each site on the day of testing is another form of the threat of selection. 
This threat to internal validity may introduce biases that result from unknown but preexisting differences between groups (Polit \& Beck, 2004). Each aspect of this threat was controlled by the analysis of demographic data about subjects as covariates and an additional variable "Site" for homogeneity of variance (Tables $8 \& 9$ ). Homogeneity of variance was confirmed for all covariates as well as for the sites, reducing the threat of selection.

Another threat to internal validity, that of a testing effect, may also impact this research. This threat arises when subjects are tested on multiple occasions, as with pretest - posttest designs. This project controlled for the effect of testing through the use of the Solomon four-group design. This form of experimental design assesses the potential of pretest sensitization separate from the effect of the treatment, in this case, the presentation of a trigger film. McGahee and Tingen (2009) illustrate that the interaction of pretest sensitization has the potential to reduce the generalizability of the outcome (McGahee, T. W. \& Tingen, M. S., 2009). Selection of this design provides an additional measure of external validity to a design already strong in internal validity (Braver, M.C.W. and Braver, S. L., 1988). A limitation of this design, stated by Braver and Braver (1988), "...is the lack of certainty concerning the proper statistical treatment of this rather complicated design." (Braver, M.C.W. and Braver, S. L., 1988). When selecting a statistical test for this design, it should be noted that no single procedure may make use of all six observations simultaneously. The asymmetric design precluded traditional methods of statistical analysis (Campbell, D.T. and Stanley, J. C., 1963). Due to this limitation, the planned profile analysis (MANOVA for repeated measures) was not 
feasible. Through a complex series of ANOVA for repeated measures recommended by Braver and Braver (1988), the data was analyzed carefully for pretest sensitivity and any effect of the treatment. Results of this analysis showed no evidence of pretest sensitivity, reducing the likelihood of the effect of testing on the results of either research question. In addition to the enhanced generalizability of the Solomon four-group design (McGahee, T. W. \& Tingen, M. S., 2009), measures were taken to reduce the threat of expectancy. A threat to external validity, the threat of expectancy (the Hawthorne effect), may take several forms: novelty effects, interaction of treatment and history effects, experimenter effects, and measurement effects. Of these potential threats, this research was at risk for only measurement effects, due to the repeated measurements at each site. To control for this threat, several measures were taken. In this research, a detailed explanation of the study provided in advance to all potential subjects would have introduced the threat of expectancy, and waiver of informed consent was approved by both the VCU Investigational Review Board (IRB), as well as the IRB of Mary Washington Hospital. Practitioners were not informed of the dates or times of data collection, and the researcher did not attempt to influence the assignment of individual subjects to particular dates or cases. This methodological approach also eliminated the potential threat of maturation on the internal validity of the study. As an additional measure, significant time was allowed to elapse between sampling periods. During each sampling period, data was collected as efficiently as possible to further reduce the threat of expectancy. 


\section{Effect Size}

Based upon the lack of a theoretically well-defined effect size and the inability to discern an effect size from the published research, a "moderate" effect size (.3) was chosen for a priori calculations of power analysis. Analysis of the data collected by this study revealed a partial-eta squared value (an indicator of effect size, see Table 13) (Field, 2005) between .001 (High Systolic Blood pressure) and .014 (Low End-Tidal Carbon Dioxide). These values indicate that a more appropriate effect size for the evaluation of accuracy of recall of patient variables is considerably less than the suggested value of "moderate" (.3). Results of this research indicate that the size of the effect of method of recordkeeping on the accuracy of recall of practitioners is extremely small, and future research in this area should account for this value when conducting calculations of power analysis and recommended sample size.

\section{Implications for Action}

Beginning with the first known account of anesthesia recordkeeping in 1894, handwritten records have sought to improve the safety and efficacy of practitioners in the care of their patients, and provide archival records for subsequent clinical cases and research purposes. With increasing monitoring capabilities, patient safety has improved, at the cost of increasing demands of recordkeeping for anesthesia providers. As early as 1934, Dr. McKesson expressed concern that the amount of recordkeeping tasks exceeded the ability of a human practitioner to both maintain appropriate vigilance to patient care while creating an accurate and timely anesthesia record. 
With these concerns in mind, development of an electronic means to record data from an anesthetic was initiated, although this goal exceeded the capabilities of technology until more modern times. As technology advanced to the point that a complete and accurate recording of multiple patient variables and events of anesthesia and surgery was possible, many experts raised voices of dissention, concerned that removal of the handwritten record would disconnect the practitioner from the flow of data critical to safe patient care (Lees, 1985; T. N. Noel, 1986; Rosen \& Rosenzweig, 1985b; Saunders, 1990). Others made the claim that removal of the human element could produce a record that was more accurate and of higher quality, as data collection could be increased through electronic means that would far exceed the capability of a handwritten chart created by a practitioner also engaged in patient care (Gravenstein et al., 1989). Perhaps the greatest concerns focused on potential for the loss of attentiveness to the patient (Kay \& Neal, 1986), and studies to compare the effects of methods of recordkeeping on the vigilance of anesthesia providers sought to respond. Often, these studies were flawed, reducing the validity of results that have become widely disseminated.

The current research effort sought to produce a simple measurement of attentiveness through the assessment of the accuracy of recall of practitioners utilizing either an AIMS recordkeeping system or a MERS. A multi-center study was proposed, in an attempt to avoid the flaw of inadequate sample size noted in previous studies, and to enhance external validity. In addition, a Solomon four-group design was implemented, to assess the degree, if any, of pretest sensitivity on the outcome of repeated measures. An 
effort was also made to address the lack of a theoretically well-defined effect size from existing literature, to ensure adequate sample size for sufficient research power. In recognition of the significant benefits and financial incentives associated with AIMS, a secondary purpose of this research was to evaluate the effect of a educational trigger film presentation on the accuracy of practitioners' recall of intraoperative patient variables. If effective, this form of education could be presented to sites currently utilizing AIMS as well as to those considering the risks and benefits of computerized recordkeeping.

\section{Results}

A total of 214 subjects participated in this research project, far in excess of the 175 subjects suggested by the power analysis for the first research question. Of these subjects, a total of 127 were analyzed to determine the potential effects of pretest sensitivity or treatment by trigger film education. Threats to internal and external validity were controlled through choice of research design, analysis of homogeneity of variance for both demographic data of participants as well as between the multiple sites. Dates for data collection were not shared with participants, and collection of data was completed as efficiently as possible to reduce the threat of expectancy.

At the conclusion of data analysis, the null hypothesis for research question \#1 was accepted, indicating no significant difference between practitioners' recall of patient variables when utilizing AIMS or MERS. In addition, the null hypothesis for research question \#2 was also accepted, as there was no significant evidence that the effect of the trigger film presentation had a perceptible effect on the accuracy of practitioners' recall. 
Also notable for the second research question was that there was no evidence of pretest sensitivity over the repeated data collection measurements.

Information processing theory and particularly the theories of attenuation and skilled memory help to explain the means by which practitioners may encode patient information into long term memory. This encoding and retrieval maintains a level of attention to the patient's condition throughout the course of the anesthetic, regardless of the method of recordkeeping employed. Results of this research support the belief that attentiveness may be maintained by practitioners utilizing AIMS, and handwritten records are not necessary to achieve this same level of "connectedness" to the anesthetic case.

Trigger film education has been used effectively in medical education for many years (Ber \& Alroy, 2001), and more recently in the education of nurse anesthetists (W. Hartland et al., 2003). The trigger film that was developed for this research was designed to present both benefits and limitations of each form of recordkeeping, to prevent the influence of experimenter bias (Polit \& Beck, 2004). As a result, specific consideration of accuracy of recall of patient variables was not presented, and could be integral in explaining the lack of effect of this treatment on subsequent measurements.

\section{Recommendations for Future Research}

Assessment of the accuracy of recall of patient variables has many potential implications for future research. The instrument developed for this study may be used to measure the effect of fatigue on anesthesia providers, by the comparison of recall accuracy of providers at various points in time throughout the day or night. Measurement 
of nurse anesthesia students or anesthesia residents at varying points of their education may provide information on the theory of skilled memory, as these practitioners develop from novice to expert in the field. Alteration in the elapsed time for recall, through measurements of 30,60, 90, or 120 minutes could offer insight into the length of time that patient variables may be accurately recalled by practitioners using either form of recordkeeping. Assessment of recall accuracy at facilities who are planning an implementation of an AIMS at intervals prior to implementation, and at 6 months and 1 year following implementation may produce enlightening results.

Utilization of existing data could produce information on attributes of practitioners who more accurately recall specific variables, or which variables are recalled most accurately. Information may be presented to the sites that participated in this research regarding the accuracy of practitioners' recall at each facility. Publication of the effect size noted by this study would be an important addition to the body of literature on the topic of anesthesia recordkeeping.

While the trigger film produced for this study did not result in a significant improvement in recall accuracy of practitioners, presentation of this film to practitioners who hold concerns similar to those expressed in the literature may result in an alteration in some beliefs that have been adequately researched and are as yet unknown to the individual. This film may benefit those practitioners who are involved in the process of selection and/or implementation of AIMS, as a means to foster discussion among colleagues and/or administrators. 


\section{Concluding Remarks}

With well documented financial incentives (Lubarsky et al., 1997; O'Sullivan et al., 2007; Reich et al., 2006), as well as government subsidies (Egger Halbeis et al., 2008)(Ehrenfeld \& Rehman, 2010), AIMS are becoming adopted at an unprecedented rate (Egger Halbeis et al., 2008). With the goals of the federal government to enhance the use of the electronic medical record, there is an emerging belief that AIMS may soon be mandated (Paradis, 2011), with more limited choices than currently available. As assessed by this research, concerns of practitioners still reflect many of the same concerns expressed in the editorial comments of the past. Current research must focus on well designed studies that address these concerns, as well as on educational methods to prepare practitioners to best select the method of recordkeeping best suited to the needs and abilities of their anesthesia practice to best ensure the safety of patients of both present and future generations. 


\section{REFERENCES}

AANA - scope and standards for nurse anesthesia practice. (2007). Retrieved 3/31/2008, 2008, from http://www.aana.com/Resources.aspx?ucNavMenu_TSMenuTargetID=51\&ucNavMen $\underline{\mathrm{u} \text { TSMenuTargetType }=4 \& \mathrm{ucNavMenu} \text { TSMenuID }=6 \& \mathrm{id}=783}$

Abenstein, J. P., DeVos, C. B., Tarhan, A., \& Tarhan, S. (1992). Eight year's experience with automated anesthesia record keeping: Lessons learned - new directions taken. International Journal of Clinical Monitoring and Computing, 9, 117-129.

Abouleish, A. E., \& Conlay, L. (2006). Automated anesthesia charge capture and submission: Wave of the future, or bridge to nowhere? Anesthesiology, 105(1), 5-7.

Allard, J., Dzwonczyk, R., Yablok, D., Block, F. E., \& McDonald, J. S. (1995). Effect of automatic record keeping on vigilance and record keeping time. British Journal of Anaesthesia, 74, 619-626.

American Association of Nurse Anesthetists. (2007). Certified registered nurse anesthetists at a glance. Retrieved 10/12, 2007, from http://www.aana.com/aboutaana.asp American Association of Nurse Anesthetists. (2008). Quality of care in anesthesia. Retrieved 3/31/2008, 2008, from 
http://www.aana.com/Resources.aspx?ucNavMenu TSMenuTargetID=51\&ucNavMen u_TSMenuTargetType $=4 \& u c N a v M e n u \_$TSMenuID $=6 \& \mathrm{id}=180$

Apple, H. P., Schneider, A. J., \& Fadel, J. (1982). Design and evaluation of a semiautomatic anesthesia record system. Medical Instrumentation, 16(1), 69-71.

ASA. (2005). Standards, guidelines and statements. Retrieved 3/31/2008, 2008, from http://www.asahq.org/publicationsAndServices/sgstoc.htm

ASA House of Delegates. (2005). Standards for basic anesthetic monitoring. Park Ridge, Illinois: American Society of Anesthesiologists.

Atkinson, R. C., \& Shiffrin, R. M. (1968). Human memory: A proposed system and its control processes. In W. K. Spence, \& J. T. Spence (Eds.), The psychology of learning and motivation: Advances in learning and theory (pp. 89-195). New York: Academic Press.

Baddeley, A. D., \& Hitch, G. (1974). Working memory. In G. H. Bower (Ed.), The psychology of learning and motivation: Advances in research and theory (8th ed., pp. 47-89). New York: Academic Press.

Beecher, H. K. (1920). The first anesthesia records (codman, cushing). Surgery, Gynecology, and Obstetrics, 71, 689-693.

Benson, M., Junger, A., Fuchs, C., Quinzio, L., Bottger, S., \& Hempelmann, G. (2000). Use of an anesthesia information management system (AIMS) to evaluate the physiologic effects of hypnotic agents used to induce anesthesia. Journal of Clinical Monitoring and Computing, 16(3), 183-190. 
Ber, R., \& Alroy, G. (2001). Twenty years of experience using trigger films as a teaching tool. Academic Medicine : Journal of the Association of American Medical Colleges, $76(6), 656-658$.

Biddle, C., Bauer, L., Dosch, M., Dove, C., Nagelhout, J., Waugaman, W., et al. (2001). Analysis of noteworthy indicators on the anesthesia record: A prospective, multiregional study. AANA Journal, 69(5), 407-410.

Block, F. E., Jr. (1991). Normal fluctuation of physiologic cardiovascular variables during anesthesia and the phenomenon of "smoothing.". Journal of Clinical Monitoring, 7(2), 141-145.

Block, F. E., Jr., Burton, L. W., Rafal, M. D., Burton, K., Newey, C., Dowell, L., et al. (1985). Two computer-based anesthetic monitors: The duke automatic monitoring equipment (DAME) system and the microDAME. Journal of Clinical Monitoring, 1(1), 30-51.

Block, F. E., Jr, Reynolds, K. M., \& McDonald, J. S. (1998). The diatek arkive "organizer" patient information management system: Experience at a university hospital. Journal of Clinical Monitoring and Computing, 14(2), 89-94.

Braver, M.C.W. and Braver, S. L. (1988). Statistical treatment of the soloman four-group design: A meta-analytic approach. Psychological Bulletin, 104(1), 150-154.

Campbell, D.T. and Stanley, J. C. (1963). Experimental and quasi-experimental designs for research. Chicago: Rand McNally.

Computer history museum - timeline of computer history. (2009). Retrieved 3/2/2009, 2009, from http://www.computerhistory.org/timeline/?category=cmptr 
Cook, R. I., McDonald, J. S., \& Nunziata, E. (1989). Differences between handwritten and automatic blood pressure records. Anesthesiology, 71(3), 385-390.

Cooper, J. B. (2007). Critical incidents, anesthesia safety and record keeping. Retrieved $3 / 31 / 2008,2008$, from http://www.apsf.org/resource center/newsletter/2000/winter/09CriticalIncidents.htm

Cooper, J. B., Newbower, R. S., Long, C. D., \& McPeek, B. (1978). Preventable anesthesia mishaps: A study of human factors. Anesthesiology, 49(6), 399-406.

Driscoll, W. D., Columbia, M. A., \& Peterfreund, R. A. (2007). An observational study of anesthesia record completeness using an anesthesia information management system. Anesthesia and Analgesia, 104(6), 1454-61, table of contents.

Driver, J. (2001). A selective review of selective attention research from the past century. British Journal of Psychology (London, England : 1953), 92 Part 1, 53-78.

Drui, A., Behm, R., \& Martin, W. (1973). Predesign investigation of the anesthesia operational environment. Anesthesia and Analgesia, 52, 584-591.

Edsall, D. W., Deshane, P., Giles, C., Dick, D., Sloan, B., \& Farrow, J. (1993). Computerized patient anesthesia records: Less time and better quality than manually produced anesthesia records. Journal of Clinical Anesthesia, 5(4), 275-283.

Edsall, D. W., Jones, B. R., \& Smith, N. T. (1992). The anesthesia database, the automated record, and the quality assurance process. International Anesthesiology Clinics, 30(2), 71-92.

Egger Halbeis, C. B., \& Epstein, R. H. (2008). The value proposition of anesthesia information management systems. Anesthesiology Clinics, 26(4), 665-79, vi. 
Egger Halbeis, C. B., Epstein, R. H., Macario, A., Pearl, R. G., \& Grunwald, Z. (2008). Adoption of anesthesia information management systems by academic departments in the united states. Anesthesia and Analgesia, 107(4), 1323-1329.

Ehrenfeld, J. M., \& Rehman, M. A. (2010). Anesthesia information management systems: A review of functionality and installation considerations. Journal of Clinical Monitoring and Computing,

Eichhorn, J. H. (1991). Quality assurance: No longer a separate process. Journal of Clinical Monitoring, 7(1), 79-81.

Eichhorn, J. H. (1993). Effect of monitoring standards on anesthesia outcome. International Anesthesiology Clinics, 31(3), 181-196.

Eichhorn, J. H., Cooper, J. B., Cullen, D. J., Maier, W. R., Philip, J. H., \& Seeman, R. G. (1986). Standards for patient monitoring during anesthesia at harvard medical school. JAMA : The Journal of the American Medical Association, 256(8), 1017-1020.

Eichhorn, J. H., \& Edsall, D. W. (1991). Computerization of anesthesia information management. Journal of Clinical Monitoring, 7(1), 71-82.

Epstein, R. H., Dexter, F., Ehrenfeld, J. M., \& Sandberg, W. S. (2009). Implications of event entry latency on anesthesia information management decision support systems. Anesthesia and Analgesia, 108(3), 941-947.

Ericsson, K. A. and Staszewski, J. J. (1989). Skilled memory and expertise: Mechanisms of exceptional performance. In Klahr, D. and Kotovsky, K. (Ed.), Complex information processing: The impact of herbert A. simon (pp. 235-267). Hillsdale, New Jersey: Lawrence Erlbaum. 
Faul, F., Erdfelder, E., Buchner, A., \& Lang, A. G. (2009). Statistical power analyses using G*Power 3.1: Tests for correlation and regression analyses. Behavior Research Methods, 41, 1149-1160.

Feingold, A., Galindo, A., \& Feingold, H. E. (1994). Lack of documentation of severe transient bradycardia by an automated anesthesia record. Journal of Clinical Monitoring, 10(6), 382-386.

Feldman, J. M. (2004). Do anesthesia information systems increase malpractice exposure? results of a survey. Anesthesia and Analgesia, 99(3), 840-3.

Field, A. (2005). Discovering statistics using SPSS (2nd ed.). London: Sage Publications. Gaba, D. M. (1990). The present and future medicolegal importance of record keeping in anesthesia and intensive care: The case for automation. Journal of Clinical Monitoring, 6(4), 338-339.

Gage, J. S. (1992). Artifacts. M.D.Computing : Computers in Medical Practice, 9(5), 288-292.

Gage, J. S., Subramanian, S., Dydro, J. F., \& Poppers, P. J. (1990). Automated anesthesia surgery medical record system. International Journal of Clinical Monitoring and Computing, 7(4), 259-263.

Gibbs, R. F. (1989a). The present and future medicolegal importance of record keeping in anesthesia and intensive care: The case for automation. Journal of Clinical Monitoring, 5(4), 251-255. 
Gibbs, R. F. (1989b). The present and future medicolegal importance of record keeping in anesthesia and intensive care: The case for automation. Journal of Clinical Monitoring, 5(4), 251-255.

Gravenstein, J. S. (1991). The anesthetic record: By hand or by computer, its purpose then and now. Journal of Clinical Monitoring, 7(1), 75-77.

Gravenstein, J. S., de Vries, A., \& Beneken, J. E. (1989). Sampling intervals for clinical monitoring of variables during anesthesia. Journal of Clinical Monitoring, 5(1), 17-21.

Gunn, I. P. (2005). Nurse anesthesia: A history of challenge. In J. J. Nagelhout, \& K. L. Zaglaniczny (Eds.), Nurse anesthesia (3rd ed., pp. 1-29). ST. Louis, MO: Elsevier.

Hamilton, W. K. (1990). The automated anesthesic record is inevitable and valuable. Journal of Clinical Monitoring, 6, 333-334.

Hartland, W.,Jr, \& Londoner, C. A. (1997). Perceived importance of clinical teaching characteristics for nurse anesthesia clinical faculty. AANA Journal, 65(6), 547-551.

Hartland, W., Biddle, C., \& Fallacaro, M. (2003). Accessing the living laboratory: Trigger films as an aid to developing, enabling, and assessing anesthesia clinical instructors. AANA Journal, 71(4), 287-291.

Heinrichs, W. (1995). Automated anaesthesia record systems, observations on future trends of development. International Journal of Clinical Monitoring and Computing, 12(1), 17-20.

Heinrichs, W., Monk, S., \& Eberle, B. (1997). Automated anesthesia record systems. [Automatische Anasthesieprotokollsysteme] Der Anaesthesist, 46(7), 574-582. 
Hogan, W. R., \& Wagner, M. M. (1997). Accuracy of data in computer-based patient records. Journal of the American Informatics Association, 4(5), 342-355.

Huslin, A. (2009, 3/26/09). Online health data in remission. Washington Post, pp. D01. Retrieved from http://www.washingtonpost.com/wpdyn/content/article/2009/02/15/AR2009021501284.html

Innovian anesthesia - your competitive advantage. (2008). Retrieved 2/28, 2008, from http://www.draeger.com/MT/internet/EN/us/prodserv/products/inform tech/innovian/p d_innovian.jsp

Ishizawa, Y. (2011). Special article: General anesthetic gases and the global environment. Anesthesia and Analgesia, 112(1), 213-217.

Issac, S., \& Michael, W. B. (1995). Handbook in research and evaluation for education and the behavioral sciences (3rd ed.). San Deigo, CA: EdITS.

Jost, A., Junger, A., Zickmann, B., Hartmann, B., Banzhaf, A., Quinzio, L., et al. (2003). Potential benefits of anaesthesia information management systems for multicentre data evaluation: Risk calculation of inotropic support in patients undergoing cardiac surgery. Medical Informatics and the Internet in Medicine, 28(1), 7-19.

Junger, A., Hartmann, B., Benson, M., Schindler, E., Dietrich, G., Jost, A., et al. (2001). The use of an anesthesia information management system for prediction of antiemetic rescue treatment at the postanesthesia care unit. Anesthesia and Analgesia, 92(5), 1203-1209. 
Kari, A., Ruokonen, E., \& Takala, J. (1990). Comparison of acceptance and performance of automated and manual data management systems in intensive care. International Journal of Clinical Monitoring and Computing, 7, 157-162.

Kay, J., \& Neal, M. (1986). Effect of automatic blood pressure devices on vigilance of anesthesia residents. Journal of Clinical Monitoring, 2(3), 148-150.

Keenan, R. L., \& Boyan, C. P. (1991). Decreasing frequency of anesthetic cardiac arrests. Journal of Clinical Anesthesia, 3(5), 354-357.

Kennedy, P. J., Feingold, A., Wiener, E. L., \& Hosek, R. S. (1976). Analysis of tasks and human factors in anesthesia for coronary-artery bypass. Anesthesia and Analgesia, 55(3), 374-377.

Kheterpal, S., Gupta, R., Blum, J. M., Tremper, K. K., O'Reilly, M., \& Kazanjian, P. E. (2007). Electronic reminders improve procedure documentation compliance and professional fee reimbursement. Anesthesia and Analgesia, 104(3), 592-597.

Kohn, L. T., Corrigan, J. M., \& Donaldson, M. S. (1999). To err is human: Building A safer health system - institute of medicine. Washington, D.C.: National Academy Press.

Kroll, D. A. (1991). The automated record: Legal help or pandora's box? Journal of Clinical Monitoring, 7(1), 77-78.

Lees, D. E. (1985). Computerized anesthesia records may have drawbacks. Anesthesiology, 63(2), 236-237.

Lerou, J. G., Dirksen, R., van Daele, M., Nijhuis, G. M., \& Crul, J. F. (1988). Automated charting of physiological variables in anesthesia: A quantitative comparison of 
automated versus handwritten anesthesia records. Journal of Clinical Monitoring, 4(1), $37-47$.

Lesser, J. B., Sanborn, K. V., Valskys, R., \& Kuroda, M. (2003). Severe bradycardia during spinal and epidural anesthesia recorded by an anesthesia information management system. Anesthesiology, 99(4), 859-866.

Levitan, D. (2008). AIMS poised to revolutionize anesthesia care. Anesthesiology News, 34(3), 8-11.

Lindop, M. J. (1981). Monitoring of the cardiovascular system during anesthesia. International Anesthesiology Clinics, 19(1), 1-29.

Loeb, R. G. (1994). Monitor surveillance and vigilance of anesthesia residents. Anesthesiology, 80(3), 527-533.

Loeb, R. G. (1995). Manual record keeping is not necessary for anesthesia vigilance. Journal of Clinical Monitoring, 11, 9-4.

Logan, G. D. (1988). Toward an instance-based theory of automatization. Psychological Review, 95, 492-527.

Logas, W. G., McCarthy, R. J., Narbone, R. F., \& Ivankovich, A. D. (1987). Analysis of the accuracy of the anesthetic record [Abstract]. Anesthesia and Analgesia, 66(S107) Lubarsky, D. A., Gan, T. J., Glass, P. S. A., Dear, G. D., Mythen, M., Dentz, M. et al. (1996). PACU clinical outcomes and financial savings following a pharmaceutical cost containment program in anesthesia using practice guidelines [Abstract]. Anesthesia and Analgesia, 82 S285. 
Lubarsky, D. A., Sanderson, I. C., Gilbert, W. C., King, K. P., Ginsberg, B., Dear, G. L., et al. (1997). Using an anesthesia information management system as a cost containment tool. description and validation. Anesthesiology, 86(5), 1161-1169.

McGahee, T. W. \& Tingen, M. S. (2009). The use of the soloman four-group design in nursing research. Southern Online Journal of Nursing Research, 9(1) Retrieved from WwW.snrs.org

McKesson, E. I. (1934). The technique of recording the effects of gas-oxygen mixtures, pressures, rebreathing and carbon-dioxid, with a summary of the effects. Anesthesia and Analgesia, 13(1), 1-7.

Melton, A. W. (1963). Implications of short-term memory for a general theory of memory. Journal of Verbal Learning and Verbal Behavior, 2, 1-21.

Miller, G. A. (1956). The magical number seven, plus or minus two: Some limits on our capacity for processing information. Psychological Review, 63, 81-97.

Mitchell, M. M. (1982). Automated anesthesia data management and recordkeeping. Medical Instrumentation, 16(6), 279-282.

Morgan, G. E., Mikhail, M. S., \& Murray, M. J. (Eds.). (2005). Clinical anesthesiology (4th ed.). New York: McGraw - Hill.

Noel, T. N. (1986). Computerized anesthesia records may be dangerous. Anesthesiology, 60,300 .

Noel, K. R. (1991). Controversy in automated record keeping. Journal of Clinical Monitoring, 7(3), 280. 
Olmedo, E. L., \& Kirk, R. E. (1977). Maintenance of vigilance by non-task-related stimulation in the monitoring environment. Perceptual and Motor Skills, 44(3 Pt 1), $715-723$.

O'Sullivan, C. T., Dexter, F., Lubarsky, D. A., \& Vigoda, M. M. (2007). Evidence-based management assessment of return on investment from anesthesia information management systems. AANA Journal, 75(1), 43-48.

Paget, N. S., Lambert, T. F., \& Sridhar, K. (1981a). Factors affecting an anaesthetist's work: Some findings on vigilance and performance. Anaesthesia and Intensive Care, 9(4), 359-365.

Paget, N. S., Lambert, T. F., \& Sridhar, K. (1981b). Factors affecting an anaesthetist's work: Some findings on vigilance and performance. Anaesthesia and Intensive Care, $9(4), 359-365$.

Paivio, A. (1971). Imagery and verbal processes. New York: Holt, Rinehart and Winston. Paradis, M. (2011, 1/26/2011). Five things every anesthesia provider should know about AIMS.

Peterson, L., \& Peterson, M. J. (1959). Short-term retention of individual verbal items. Journal of Experimental Psychology, 58, 193-198.

Petry, A., Gockel, H., \& Wulf, H. (1993a). Computer-aided anesthesia monitoring. experiences with the use of three systems in heart surgery. [Computer-unterstutztes Narkosemonitoring. Erfahrungen beim Einsatz dreier Systeme in der Kardiochirurgie] Der Anaesthesist, 42(8), 528-535. 
Petry, A., Gockel, H., \& Wulf, H. (1993b). Computer-aided anesthesia monitoring. experiences with the use of three systems in heart surgery. [Computer-unterstutztes Narkosemonitoring. Erfahrungen beim Einsatz dreier Systeme in der Kardiochirurgie] Der Anaesthesist, 42(8), 528-535.

Pierce, E. C.,Jr. (1988). Does monitoring have an effect on patient safety? monitoring instruments have significantly reduced anesthetic mishaps. Journal of Clinical Monitoring, 4(2), 111-114.

Polit, D. F., \& Beck, C. T. (2004). Nursing research: Principles and methods (7th ed.). Philadelphia: LIppencott Williams and Wilkins.

Posner, M. I., \& Snyder, C. R. R. (1975). Facilitation and inhibition in the processing of signals. In P. M. A. Rabbitt, \& S. Dornic (Eds.), Attention and performance (pp. 669682). New York: Academic Press.

Reich, D. L., Hossain, S., Krol, M., Baez, B., Patel, P., Bernstein, A., et al. (2005). Predictors of hypotension after induction of general anesthesia. Anesthesia and Analgesia, 101(3), 622-8, table of contents.

Reich, D. L., Kahn, R. A., Wax, D., Palvia, T., Galati, M., \& Krol, M. (2006).

Development of a module for point-of-care charge capture and submission using an anesthesia information management system. Anesthesiology, 105(1), 179-86; quiz 2312.

Report brief. to err is human: Building a safer health system - institute of medicine. Retrieved 3/18/2008, 2008, from http://www.iom.edu/CMS/8089/5575/4117.aspx 
Robinson-Riegler, G., \& Robinson-Riegler, B. (2004). Cognitive psychology: Applying the science of the mind. Boston: Pearson Education, Inc.

Rosen, A. S., \& Rosenzweig, W. (1985a). Computerized anesthesia record. Anesthesiology, 62(1), 100-101.

Rosen, A. S., \& Rosenzweig, W. (1985b). In reply to computerized anesthesia records may have drawbacks. Anesthesiology, 62(2), 237.

Rosen, A. S., \& Rosenzweig, W. (1986). On computerized anesthesia records. Anesthesiology, 65(1), 131-132.

Sanborn, K. V., Castro, J., Kuroda, M., \& Thys, D. M. (1996a). Detection of intraoperative incidents by electronic scanning of computerized anesthesia records. comparison with voluntary reporting. Anesthesiology, 85(5), 977-987.

Sanborn, K. V., Castro, J., Kuroda, M., \& Thys, D. M. (1996b). Detection of intraoperative incidents by electronic scanning of computerized anesthesia records. comparison with voluntary reporting. Anesthesiology, 85(5), 977-987.

Sarnat, A. J. (1986). Do not fear computerized anesthesia records. Anesthesiology, 65(1), $132-133$.

Saunders, R. J. (1990). The automated anesthetic record will not automatically solve problems in record keeping. Journal of Clinical Monitoring, 6, 334-337.

Schneider, W., \& Shiffrin, R. M. (1977). Controlled and automatic human information processing, I: Detection, search, and attention. Psychological Review, 84, 1-66. 
Shaffer, M. J., Kaiser, P. R., Klingenmaier, C. H., \& Gordon, M. R. (1978). Manual record-keeping and statistical records for the operating room. Medical Instrumentation, 12(3), 192-197.

Shelley, K. (2008). Is the automation of anesthesia possible or even desirable? Current Opinion in Anaesthesiology, 21(6), 748-749.

Shibutani, K., Bairamian, M., Subhedar, D., Kashiwagi, N., \& Kubal, K. (1990). Human factors affecting accuracy of hand-written arterial pressure record [Abstract]. Anesthesiology, 73(A1014)

Smith, N. T. (1991). Future possibilities. Journal of Clinical Monitoring, 7(1), 81-82. Sperling, G. (1960). Theinformation available in brief visual presentation. Psychological Monographs, 74, 1-29.

Spring, S. F., Sandberg, W. S., Anupama, S., Walsh, J. L., Driscoll, W. D., \& Raines, D. E. (2007). Automated documentation error detection and notification improves anesthesia billing performance. Anesthesiology, 106(1), 157-163.

Sticher, J., Junger, A., Hartmann, B., Benson, M., Jost, A., Golinski, M., et al. (2002). Computerize anesthesia record keeping in thoracic surgery--suitability of electronic anesthesia records in evaluating predictors for hypoxemia during one-lung ventilation. Journal of Clinical Monitoring and Computing, 17(6), 335-343.

Stoelting, R. K. (2001). President reports on state of APSF. Retrieved 3/31/2008, 2008, from http://www.apsf.org/resource_center/newsletter/2002/winter/02presidentreports.htm 
Tabachnick, B. G., \& Fidell, L. S. (2007). Using multivariate statistics (5th ed.). Boston: Pearson.

Thatcher, V. S. (1953). History of anesthesia : With emphasis on the nurse specialist. Philadelphia: Lippincott.

Thompson, P. W. (1978). Safety of anaesthetic apparatus. International Anesthesiology Clinics, 16(1), 199-223.

Thrush, D. N. (1992). Are automated anesthesia records better? Journal of Clinical Anesthesia, 4(5), 386-389.

Thys, D. M. (1998). The role of information systems in anesthesia. Retrieved 3/31/2008, 2008, from http://www.apsf.org/resource_center/newsletter/2001/summer/03Infosys.htm

Treisman, A. (1960). Contextual cues in selective listening. Quarterly Journal of Experimental Psychology, 12, 242-248.

Tulving, E. (1972). Episodic and semantic memory. In E. Tulving, \& W. Donaldson (Eds.), Organization of memory (). New York: Academic Press.

Vigoda, M. M., Gencorelli, F., \& Lubarsky, D. A. (2006). Changing medical group behaviors: Increasing the rate of documentation of quality assurance events using an anesthesia information system. Anesthesia and Analgesia, 103(2), 390-5.

Vigoda, M. M., \& Lubarsky, D. A. (2006a). Failure to recognize loss of incoming data in an anesthesia record-keeping system may have increased medical liability. Anesthesia and Analgesia, 102(6), 1798-1802. 
Vigoda, M. M., \& Lubarsky, D. A. (2006b). The medicolegal importance of enhancing timeliness of documentation when using an anesthesia information system and the response to automated feedback in an academic practice. Anesthesia and Analgesia, 103(1), 131-6, table of contents.

Vitkun, S. A., Gage, J. S., Anderson II, D. H., Williams, S. A., Halpern-Lewis, J. G., \& Poppers, P. J. (1995). Computerization of the preoperative anesthesia interview. International Journal of Clinical Monitoring and Computing, 12, 71-76.

Wang, X., Gardner, R. M., \& Seager, P. R. (1995). Integrating computerized anesthesia charting into a hospital information system. Journal of Clinical Monitoring and Computing, 12, 61-70.

Weinger, M. B., \& Englund, C. E. (1990). Ergonomic and human factors affecting anesthetic vigilance and monitoring performance in the operating room environment. Anesthesiology, 73(5), 995-1021.

Weinger, M. B., Herndon, O. W., \& Gaba, D. M. (1997). The effect of electronic record keeping and transesophageal echocardiography on task distribution, workload, and vigilance during cardiac anesthesia. Anesthesiology, 87(1), 144-155.

Weinger, M. B., Reddy, S. B., \& Slagle, J. M. (2004). Multiple measures of anesthesia workload during teaching and nonteaching cases. Anesthesia and Analgesia, 98(5), 1419-25, table of contents.

Westenskow, D. W. (1991). Artifacts and alarms: Problems and benefits. Journal of Clinical Monitoring, 7(1), 78-79. 
Whiting-O'Keefe, Q. E., Simborg, D. W., Epstein, W. V., \& Warger, A. (1985). A computerized summary medical record system can provide more information than the standard medical record. JAMA : The Journal of the American Medical Association, 254(9), 1185-1192.

Woods, D. D., Cook, R. I., \& Billings, C. E. (1995). The impact of technology on physician cognition and performance. Journal of Clinical Monitoring, 11(1), 5-8.

Yablok, D. O. (1990). Comparison of vigilance using automated versus handwritten records [Abstract]. Anesthesiology, 73 A416.

Zbinden, A. M., Christensen, J., \& Kuster, M. (1992). How can a standard software package for data management in anesthesia be achieved? Journal of Clinical Monitoring, 8(4), 315-318.

Zollinger, R. M.,Jr, Kreul, J. F., \& Schneider, A. J. (1977). Man-made versus computergenerated anesthesia records. The Journal of Surgical Research, 22(4), 419-424. 
APPENDIX A: RECORDKEEPING SURVEY 
Recordkeeping Survey

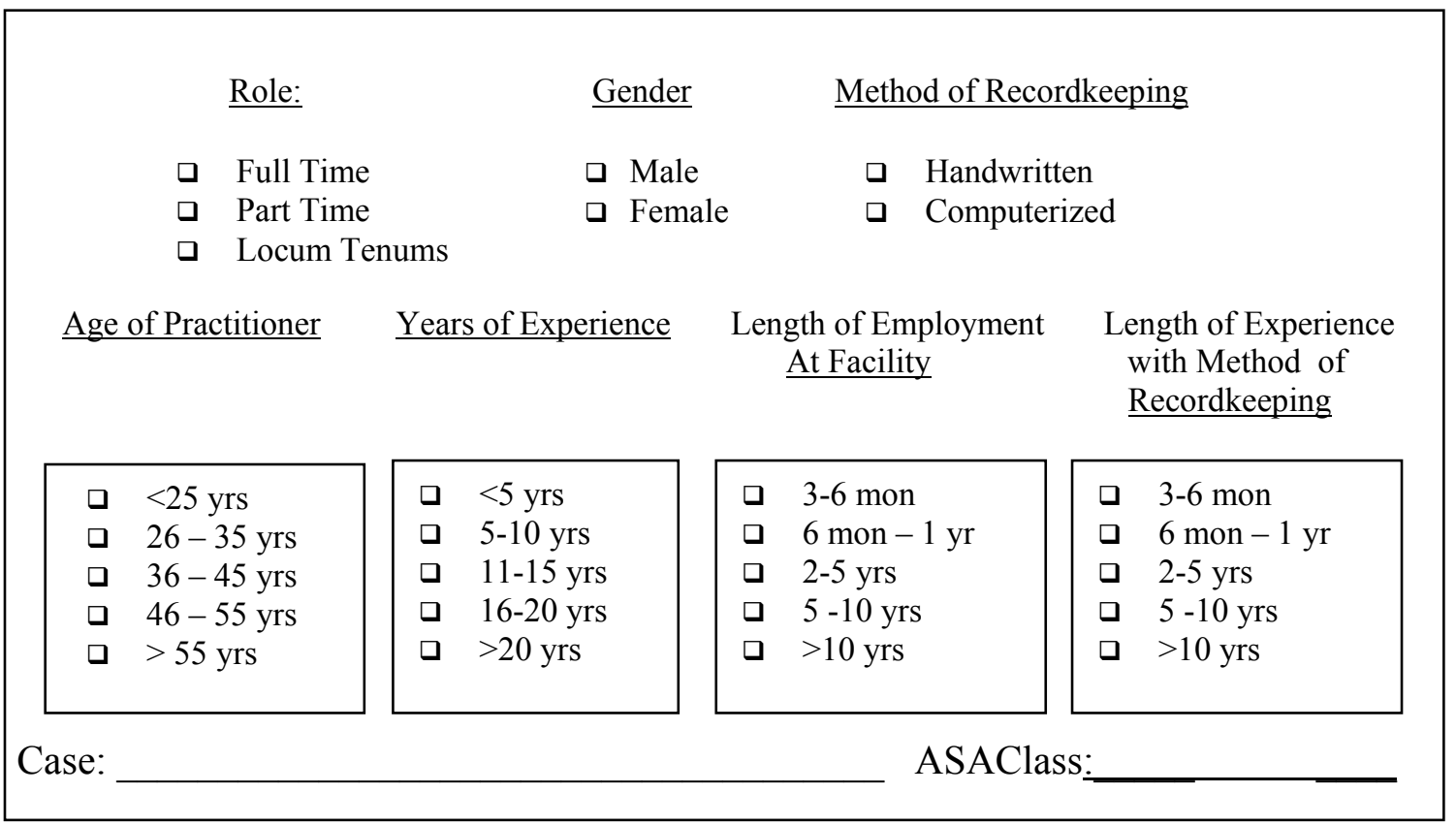

\begin{tabular}{|c|c|}
\hline Variable & Actual Value (Trends @ 5 min) \\
\hline Highest Heart Rate & \\
\hline Lowest Heart Rate & \\
\hline $\begin{array}{c}\text { Highest Systolic } \\
\text { Blood Pressure }\end{array}$ & \\
\hline $\begin{array}{c}\text { Lowest Systolic } \\
\text { Blood Pressure }\end{array}$ & \\
\hline Highest ETCO & \\
\hline Lowest ETCO & \\
\hline Lowest SPO & \\
\hline $\begin{array}{c}\text { Highest Inspiratory } \\
\text { Pressure }\end{array}$ & \\
\hline $\begin{array}{c}\text { Lowest Inspiratory } \\
\text { Pressure }\end{array}$ & \\
\hline $\begin{array}{c}\text { Total Fluid Volume } \\
\text { for case }\end{array}$ & \\
\hline
\end{tabular}


APPENDIX B: SPEARMAN'S TEST OF CORRELATION OF COVARIATES 
Spearman's Test of Correlation of Covariates

\begin{tabular}{|c|c|c|}
\hline \multicolumn{2}{|c|}{ Method of Recordkeeping } & \multicolumn{2}{|c|}{ Spearman's Test } \\
\hline MERS $=1 \quad$ AIMS $=2$ & .002 & Value \\
\hline Covariates & .008 & .209 \\
Gender & .001 & -.180 \\
Age & .113 & -.227 \\
Years of Experience & & -.109 \\
Length of Employment & .000 & -.376 \\
Years of Experience with Method of & & .041 \\
Recordkeeping & .552 & \\
ASA Classification & & \\
\hline
\end{tabular}


APPENDIX C: HISTOGRAMS OF COVARIATES BY SITE 
Histograms of Covariates by Site

- Normal

Histogram

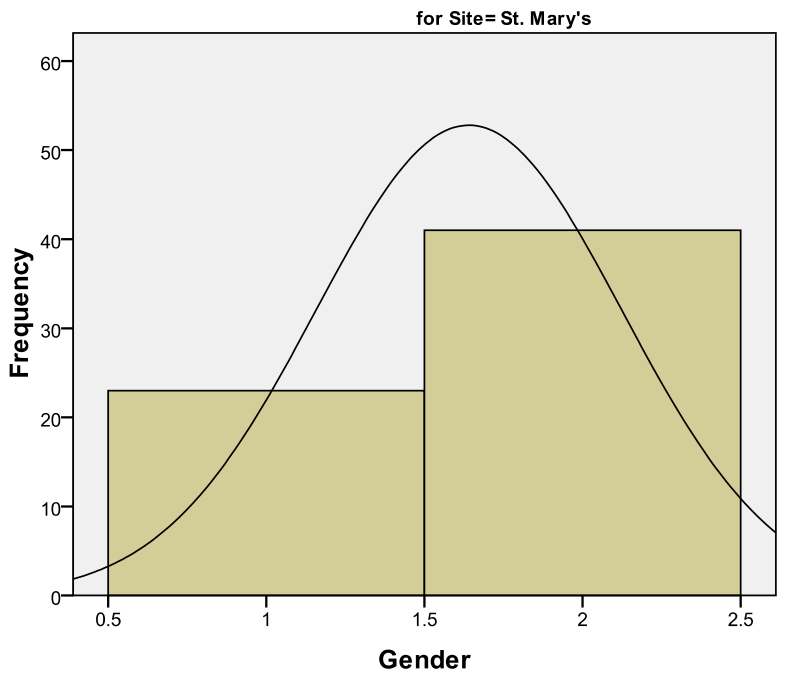
Mean $=1.64$
Std. Dev. $=0.484$
$\mathrm{~N}=64$

- Normal

\section{Histogram}

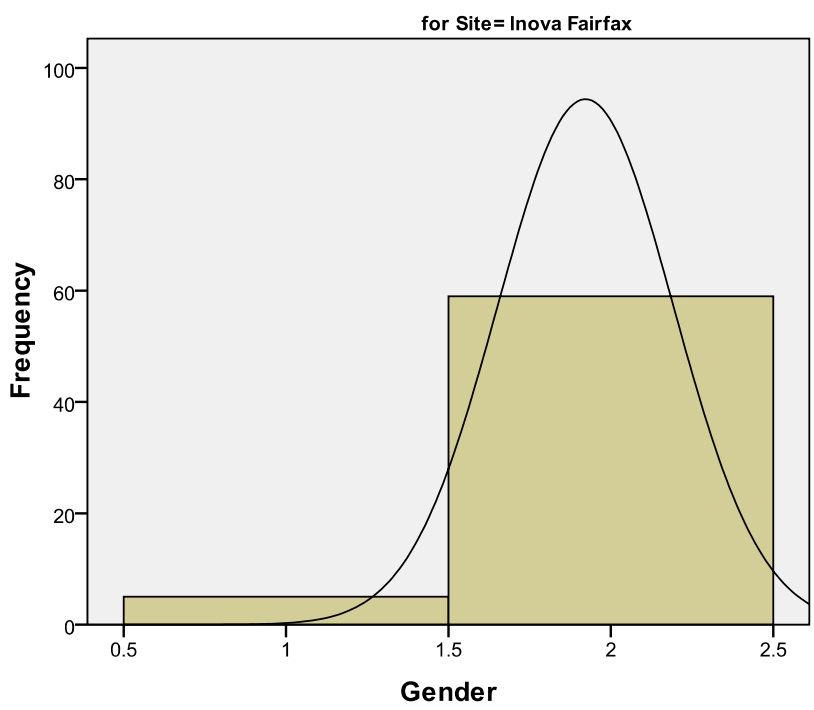

Mean $=1.92$ Dev. $=0.27$ $\mathrm{N}=64$ 
Histogram

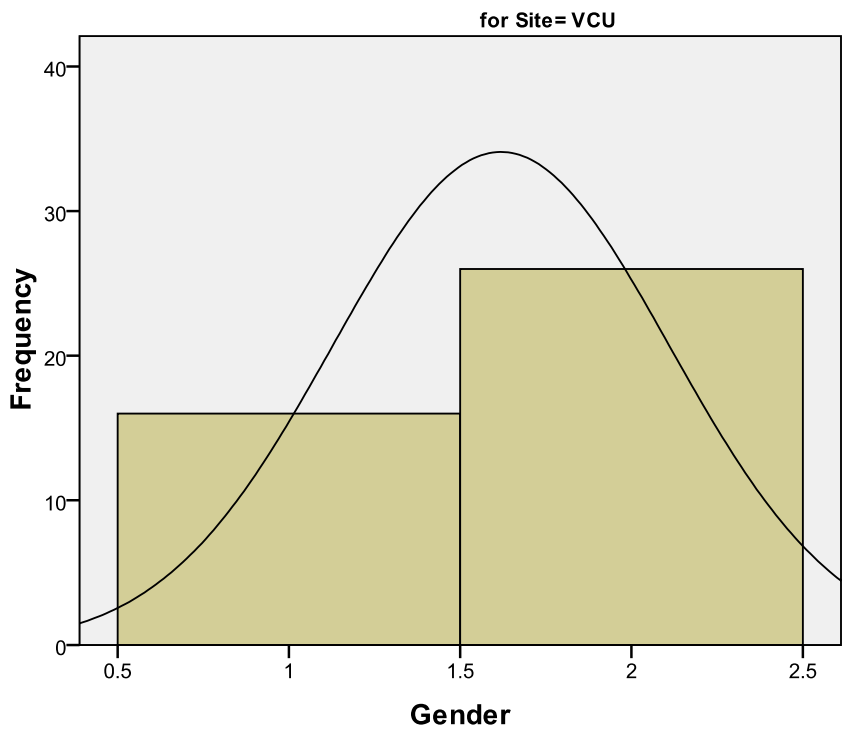
Mean $=1.62$
Std. Dev. $=0.492$ $\mathrm{N}=42$

- Normal

\section{Histogram}

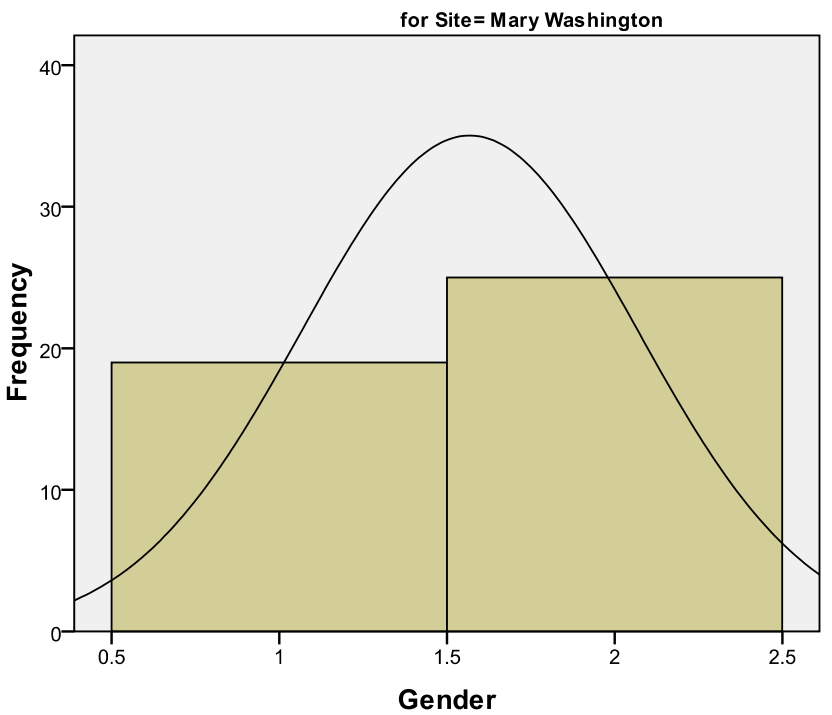

Mean $=1.57$ td. Dev. $=0.501$ 
Histogram

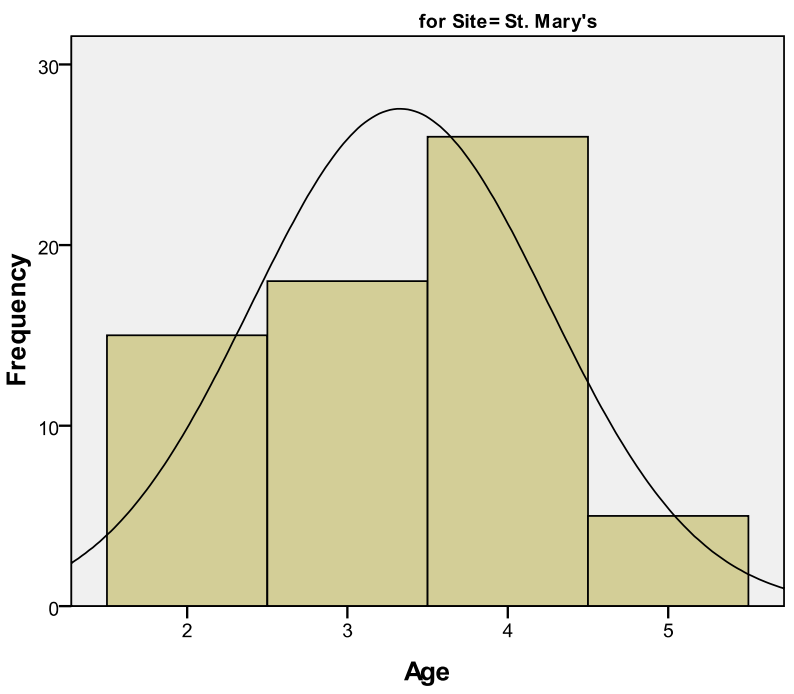

Mean $=3.33$

$\mathrm{N}=64$

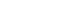

— Normal

Histogram

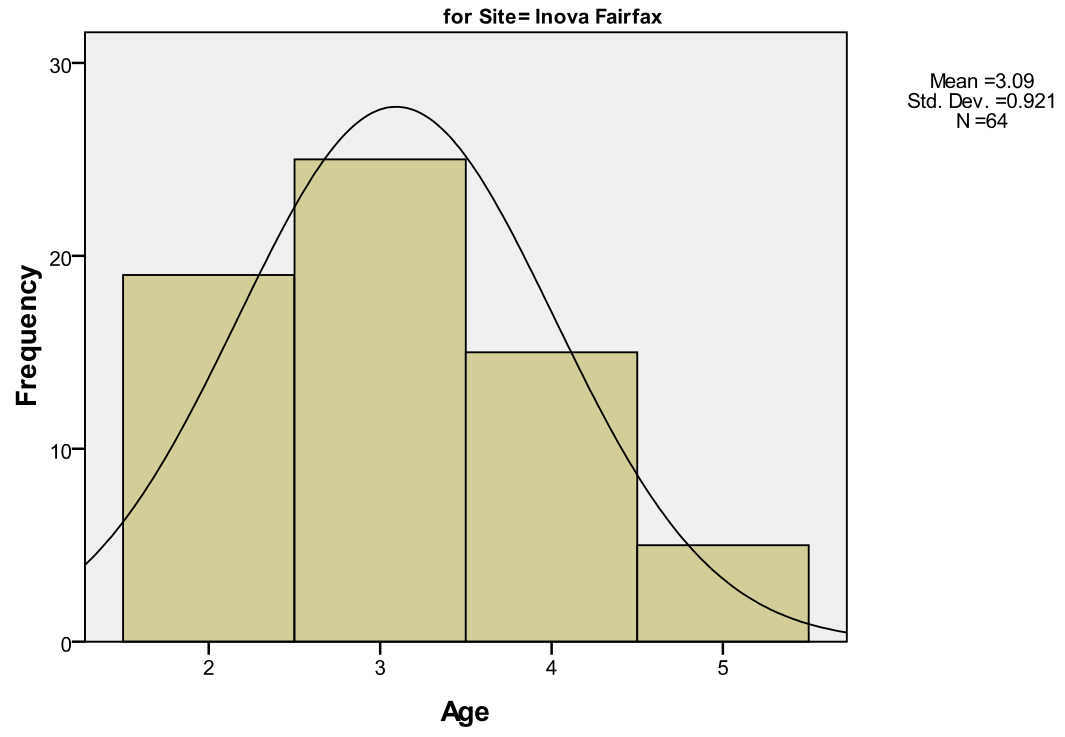


Histogram

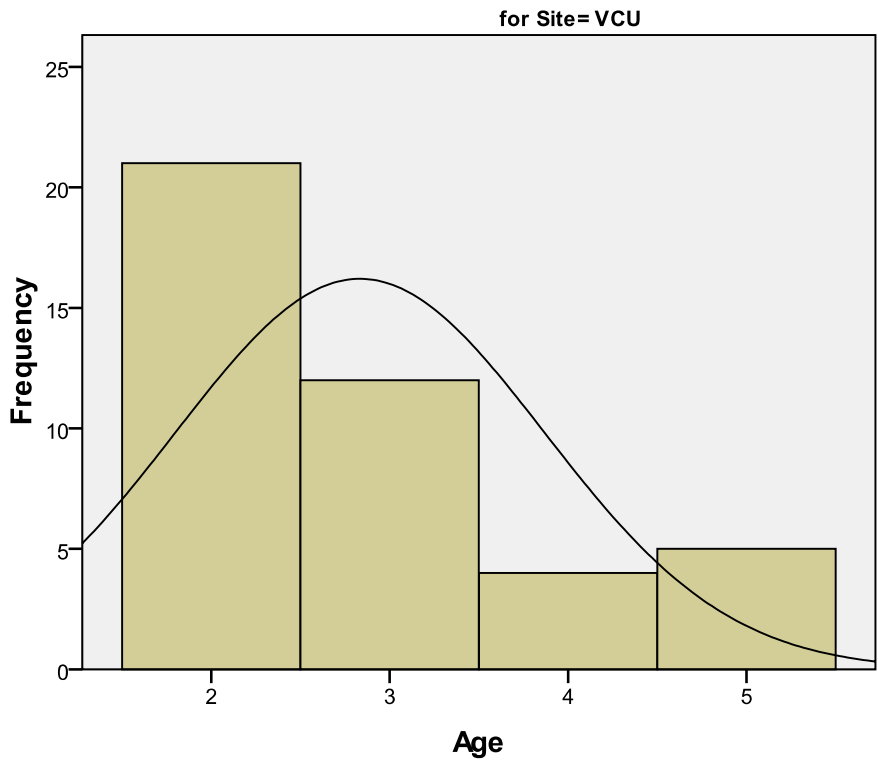

Mean $=2.83$

Std. Dev. $=1.034$
$N=42$

Histogram

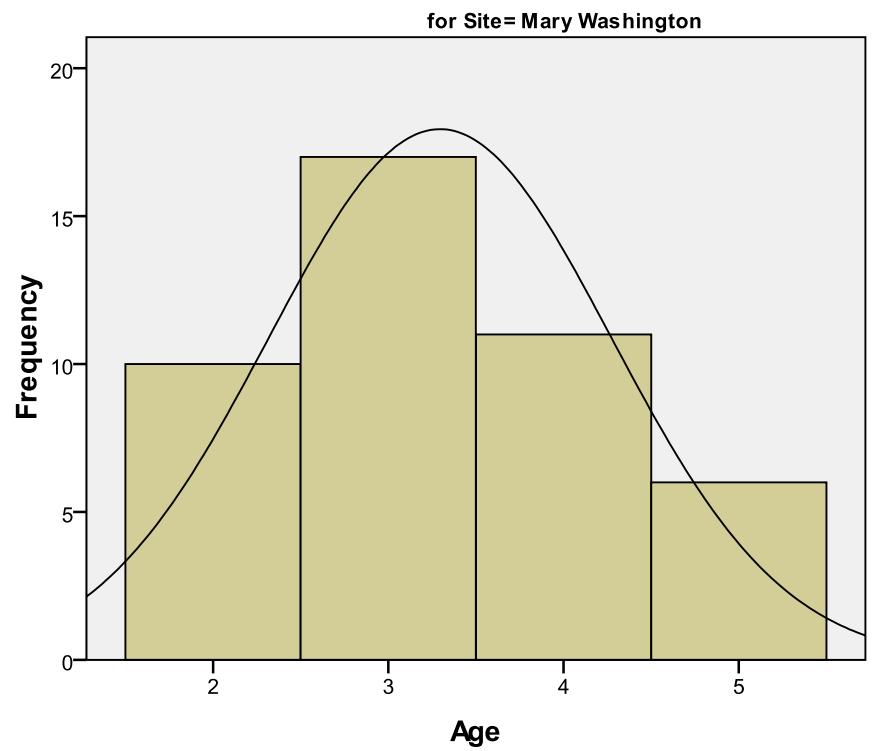

Mean $=3.3$ Std. Dev. $=0.978$

$\mathrm{N}=44$ 
Histogram

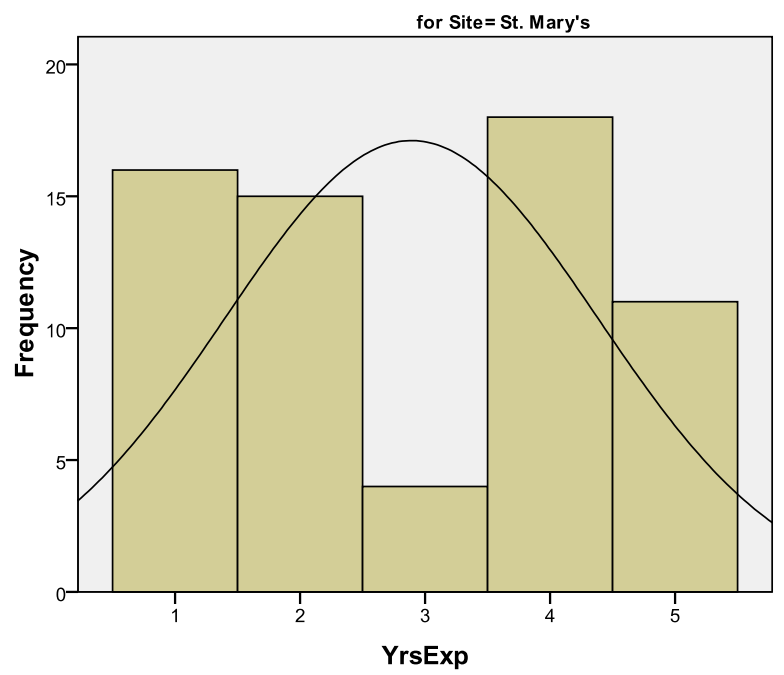

Histogram

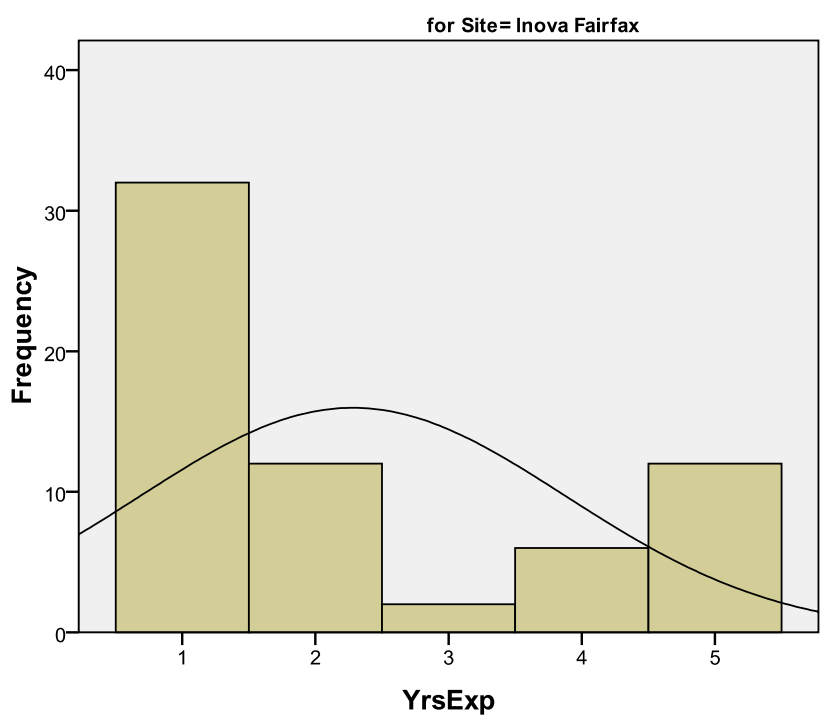

$\begin{aligned} \text { Mean } & =2.89 \\ \text { Std. Dev. }=1.492 & \end{aligned}$ $\mathrm{N}=64$
$\mathrm{~N}=\mathrm{Nev}$

- Normal

Mean $=2.28$
Std. Dev. $=1.598$ $\mathrm{N}=64$
$\mathrm{~N}=1$. 
Histogram

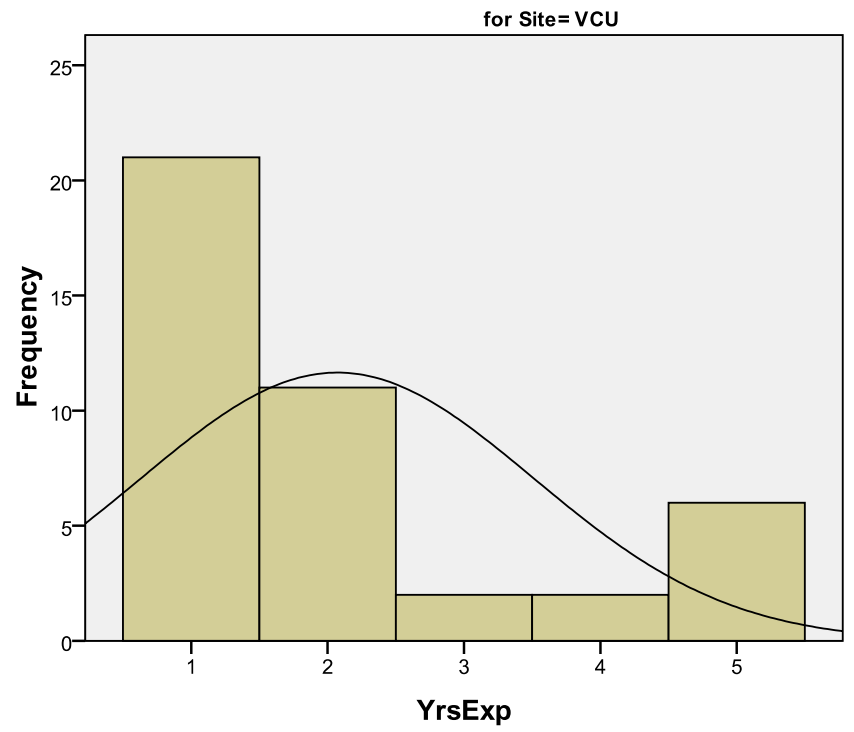

$\begin{aligned} \text { Mean } & =2.07 \\ & \end{aligned}$ d. Dev. $=1.438$

- Normal

Histogram

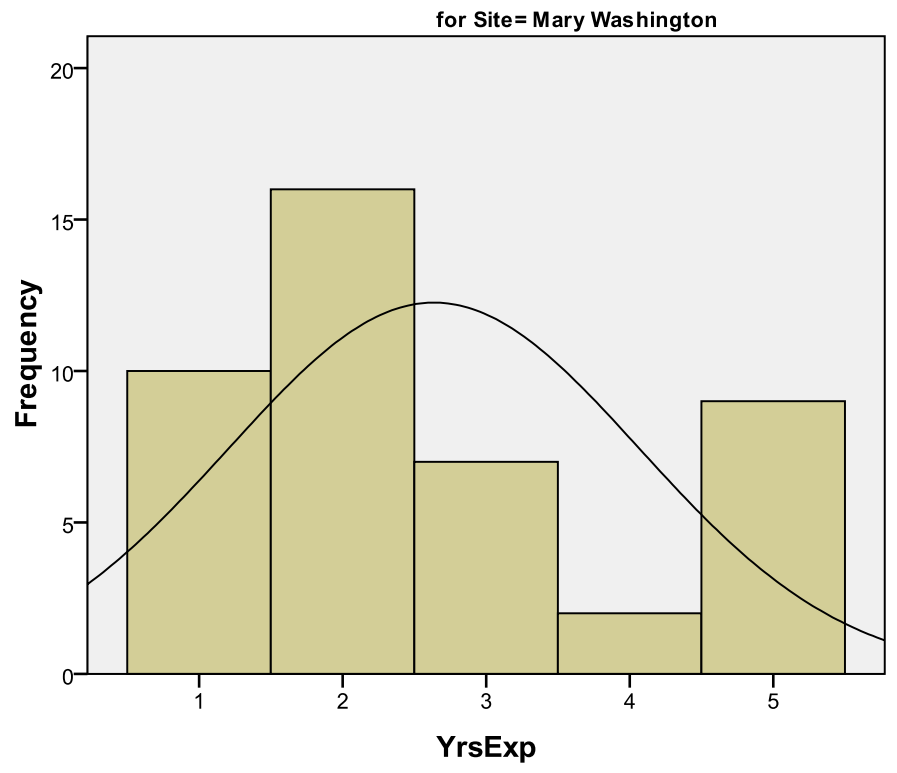

Mean $=2.64$ Std. Dev. $=1.432$ $\mathrm{N}=44$ 


\section{Histogram}

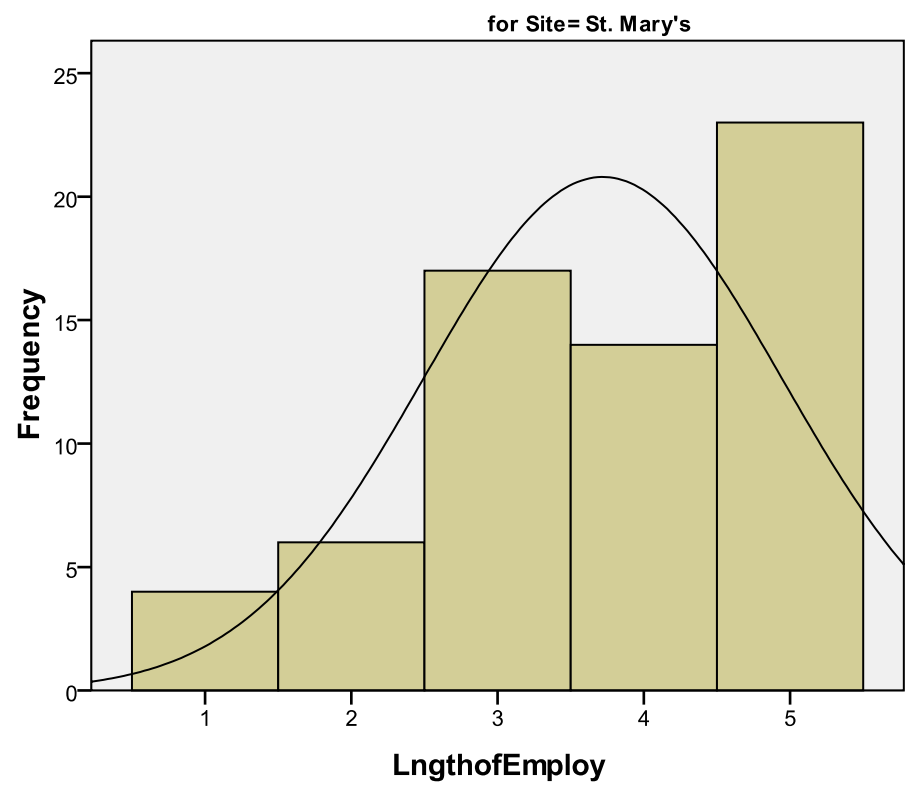

Mean $=3.72$ Std. Dev. $=1.228$ $\mathrm{N}=64$

- Normal

Histogram

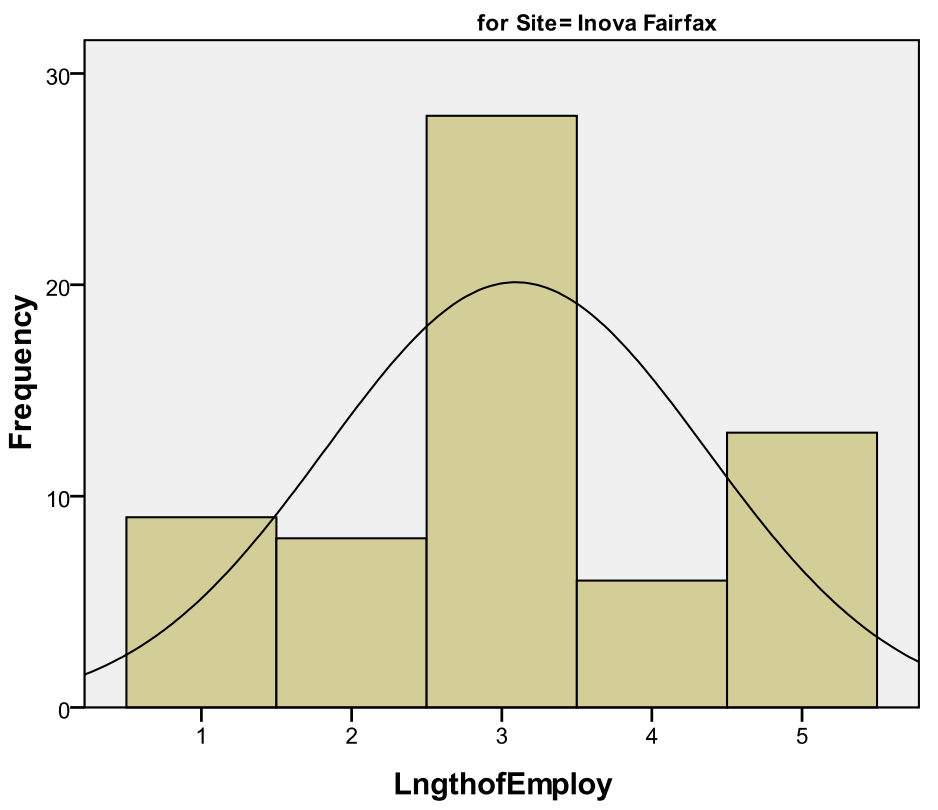

Mean $=3.09$ Std. Dev. $=1.269$ 
Histogram

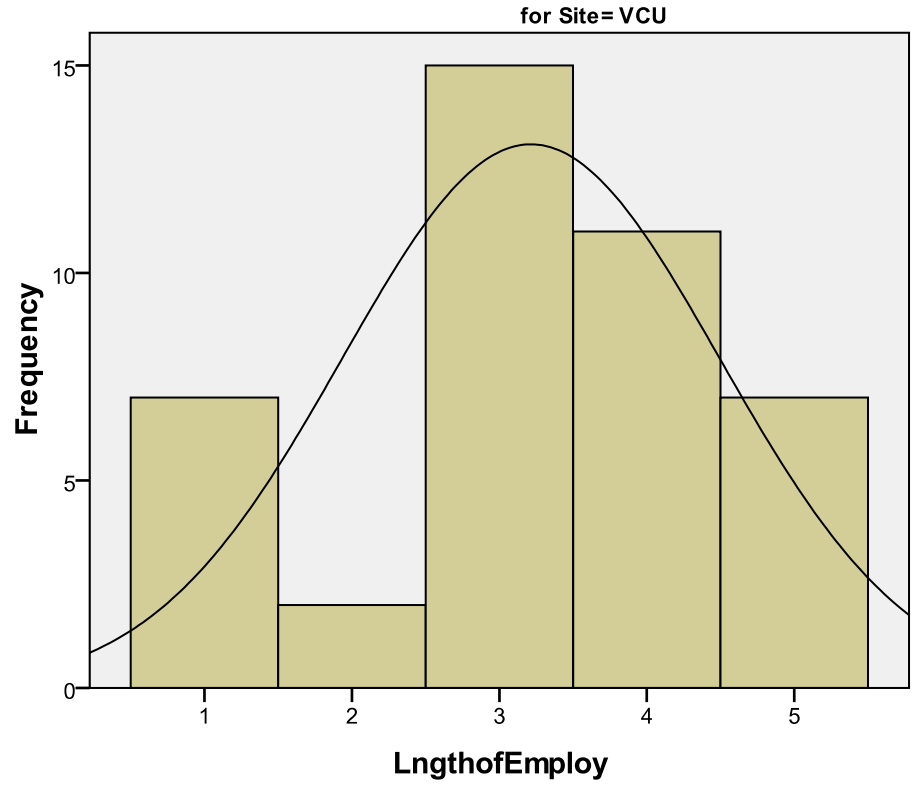
Mean $=3.21$
Std. Dev. $=1.279$
$N=42$

— Normal

Histogram

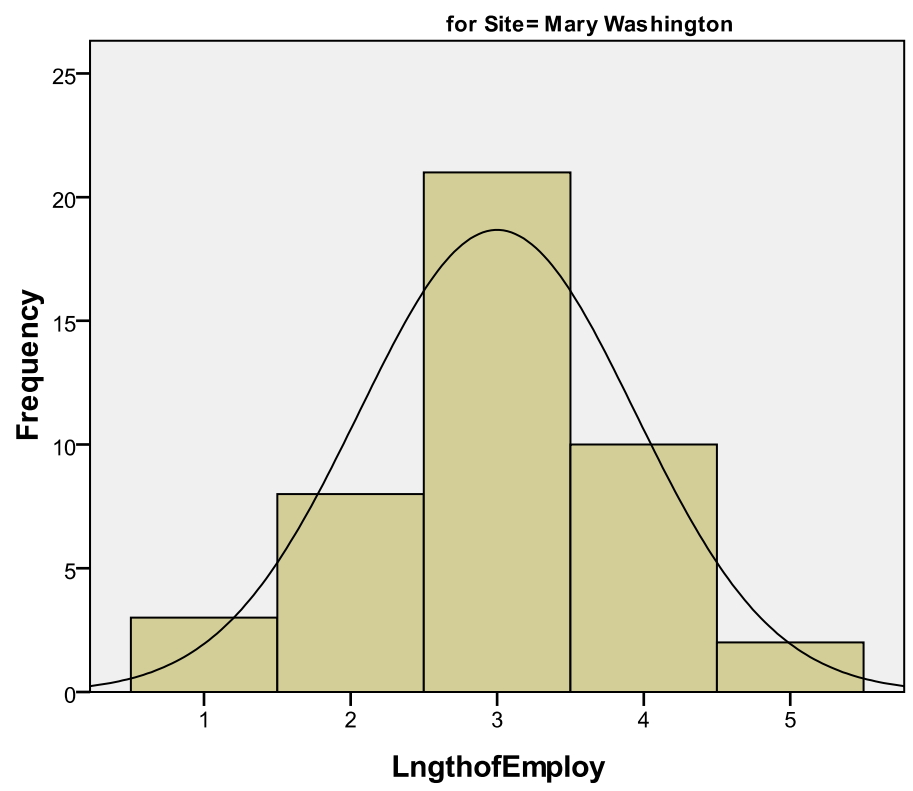

Mean $=3$ Std. Dev. $=0.94$

$\mathrm{N}=44$ 
Histogram

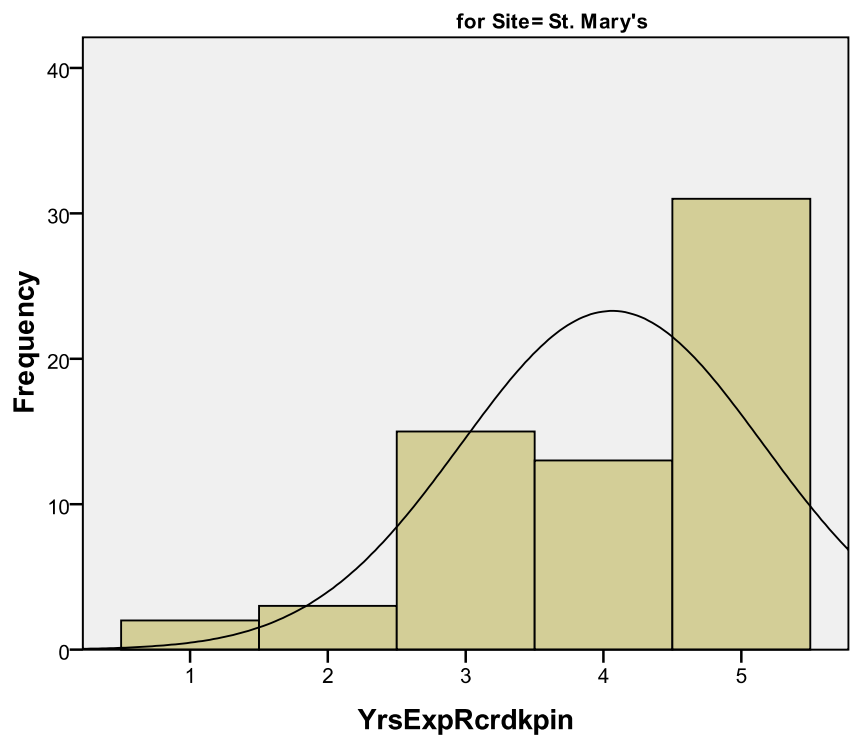

Mean $=4.06$ d. Dev. $\mathrm{N}=64$

- Normal

Histogram

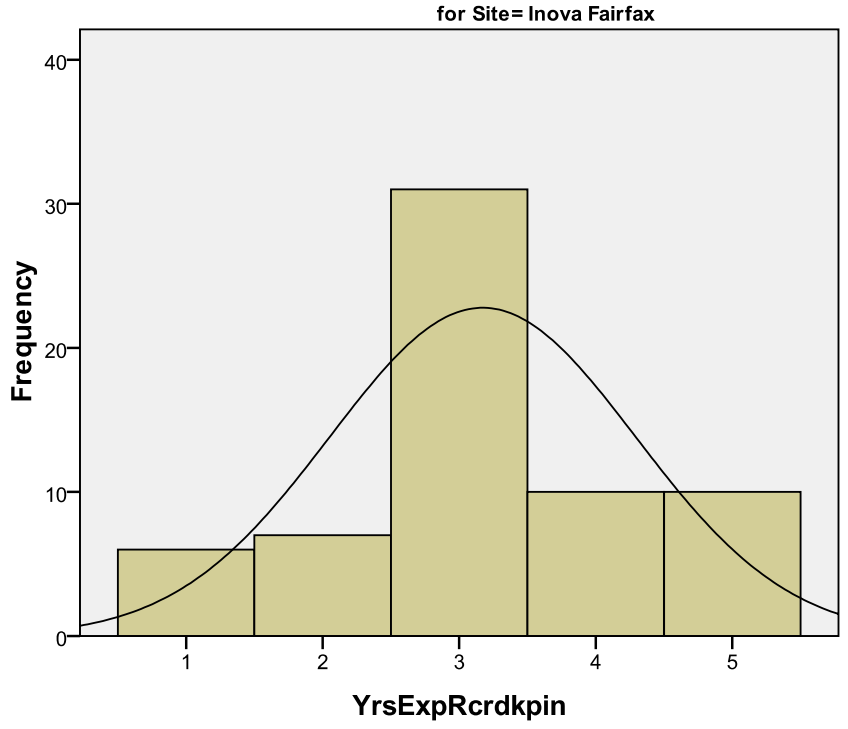

Mean $=3.17$ Std. Dev. $=1.121$ $\mathrm{N}=64$ 


\section{Histogram}

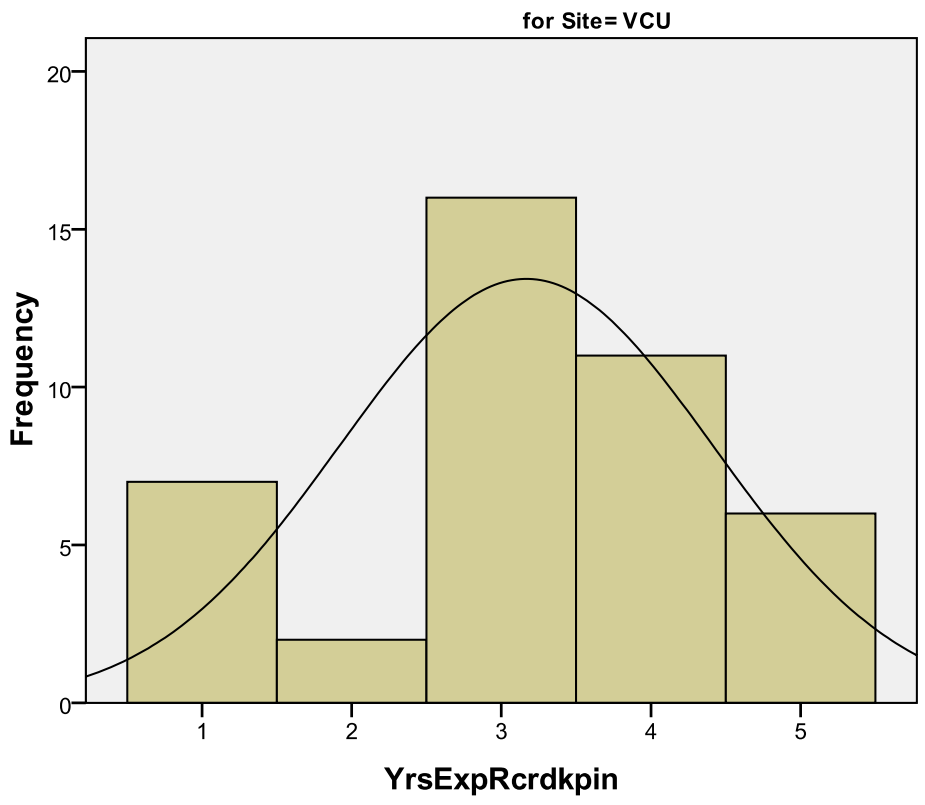

- Normal

Histogram

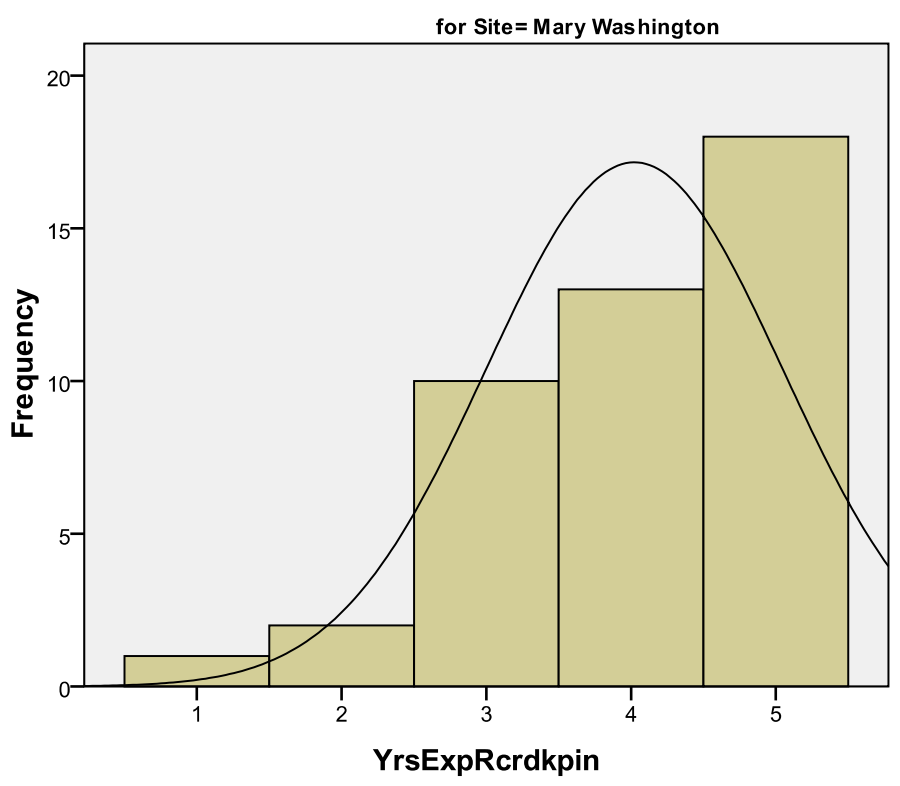

Mean $=3.17$ Std. Dev. $=1.248$ $\mathrm{N}=42$ 


\section{Histogram}

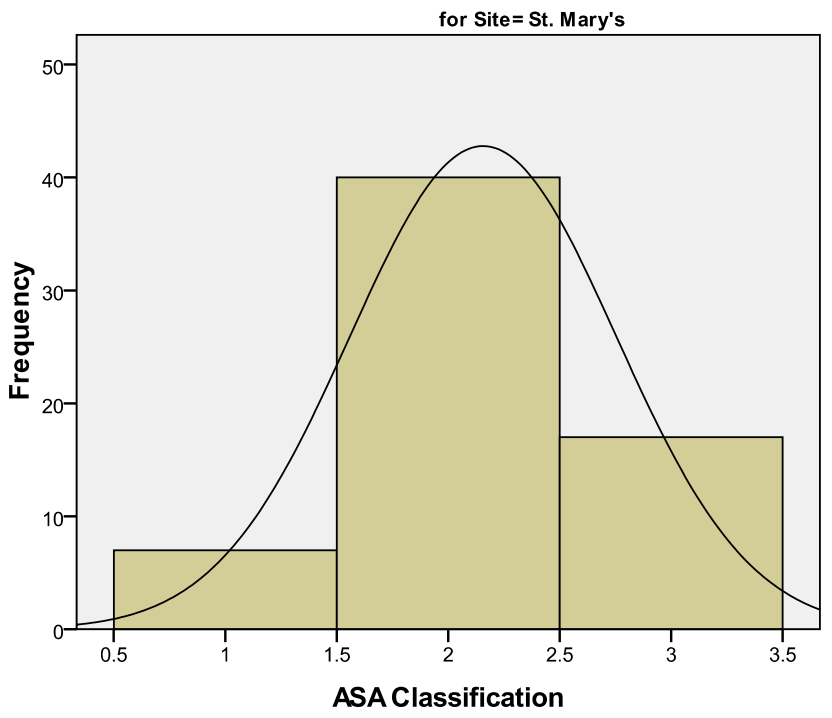

Mean $=2.16$ Std. Dev. $=0.597$ $\mathrm{N}=64$

- Normal

Histogram

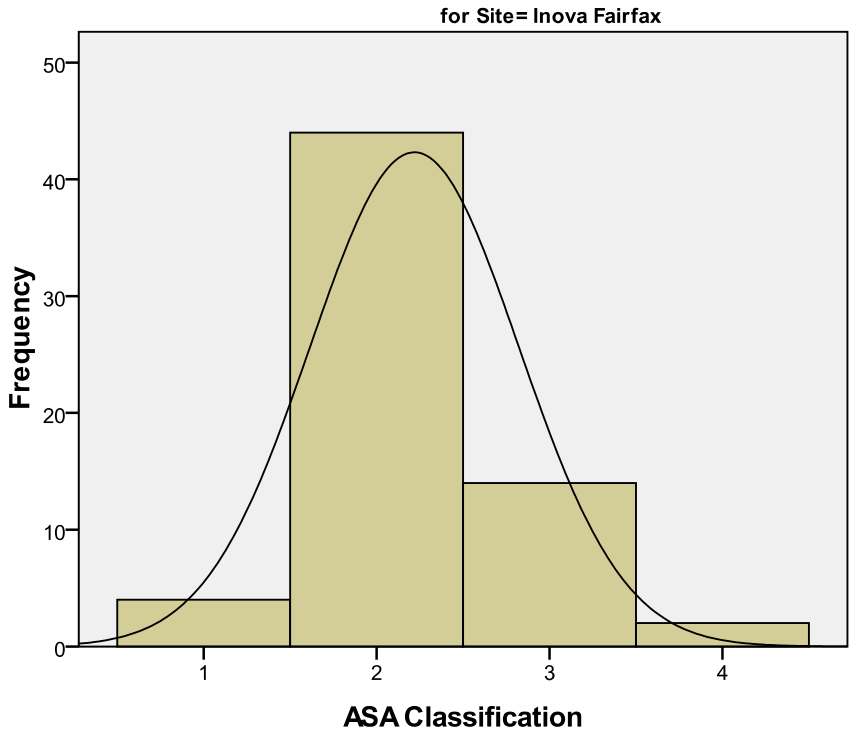

Mean $=2.22$ $\begin{aligned} \text { Std. Dev. } & =0.603 \\ N & =64\end{aligned}$ 
Histogram

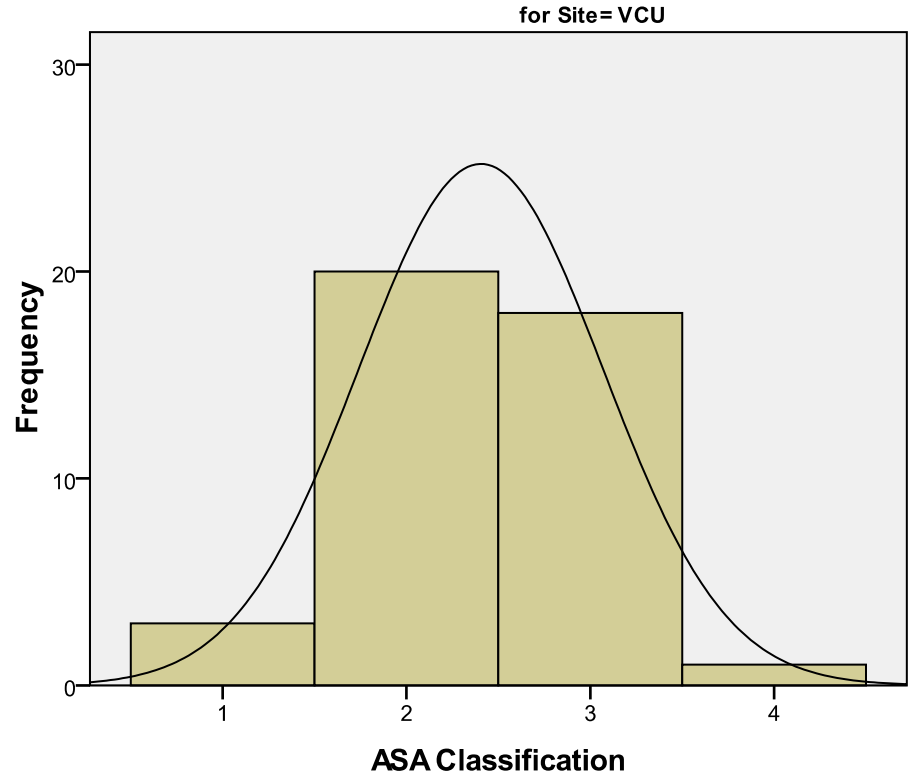
$\begin{aligned} \text { Mean } & =2.4 \\ \text { Std. Dev. } & =0.665\end{aligned}$ $\mathrm{N}=42$

- Normal

Histogram

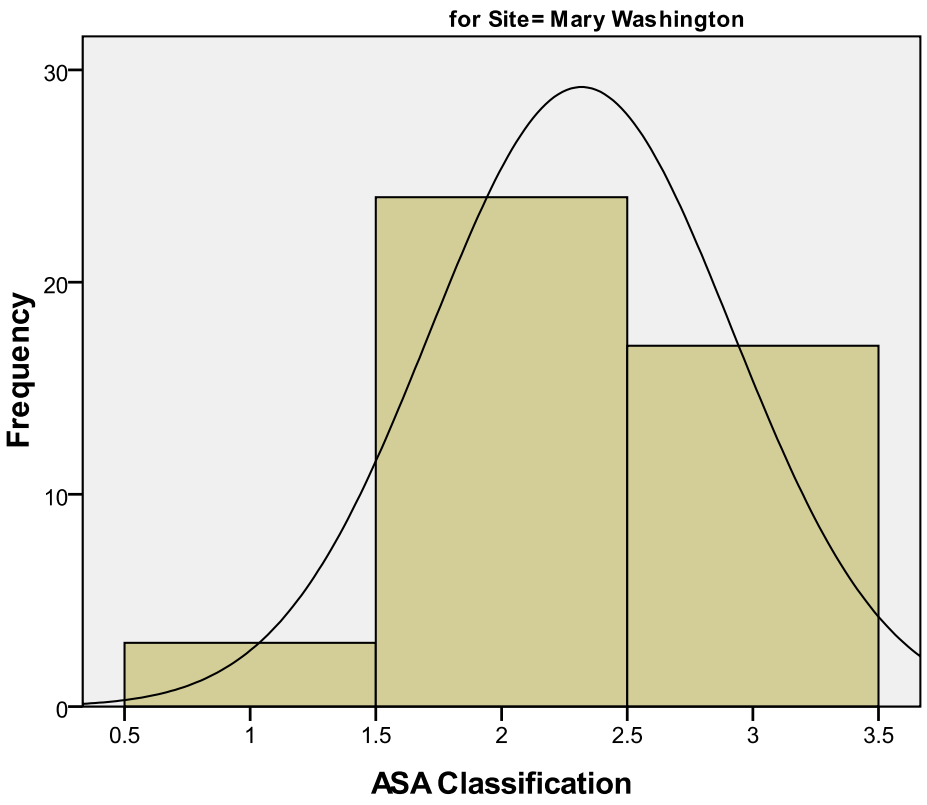

Mean $=2.32$ Std. Dev. $=0.601$ $\mathrm{N}=44$ 
APPENDIX D: HISTOGRAMS OF COVARIATES BY METHOD OF RECORDKEEPING 
Histograms of Covariates by Method of Recordkeeping

- Normal

\section{Histogram}

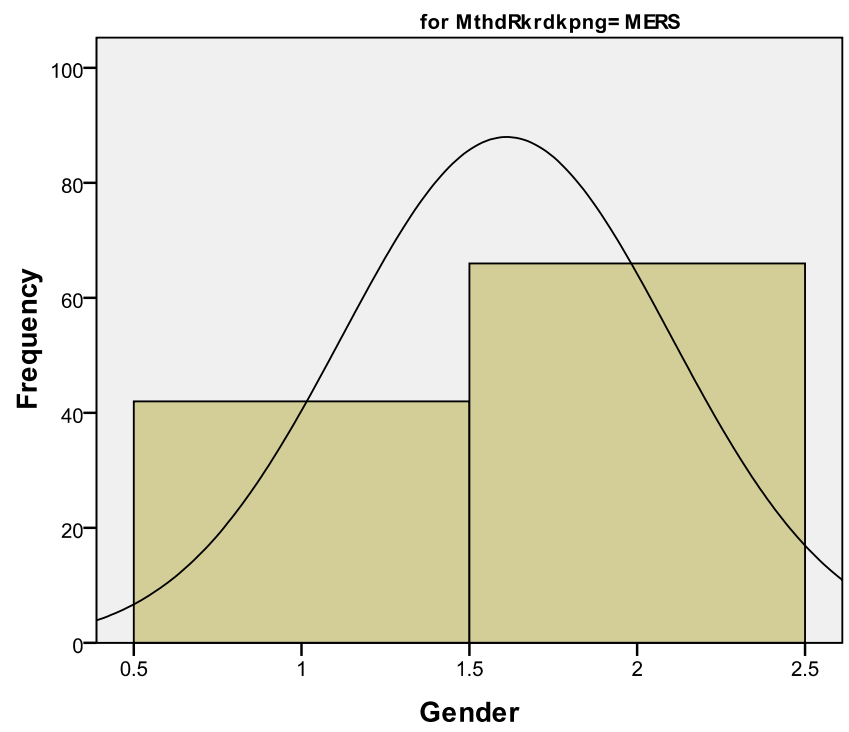

Mean $=1.61$

td. Dev. $=0.49$

— Normal

\section{Histogram}

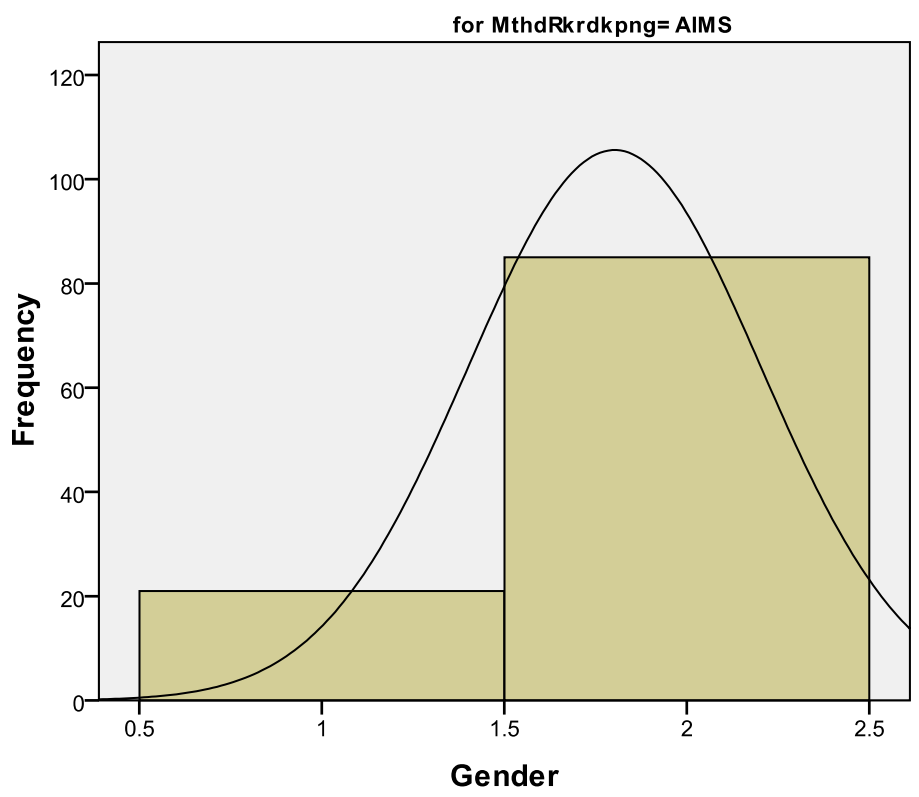

Mean $=1.8$ Std. Dev. $=0.4$ $\mathrm{N}=106$ 
- Normal

Histogram

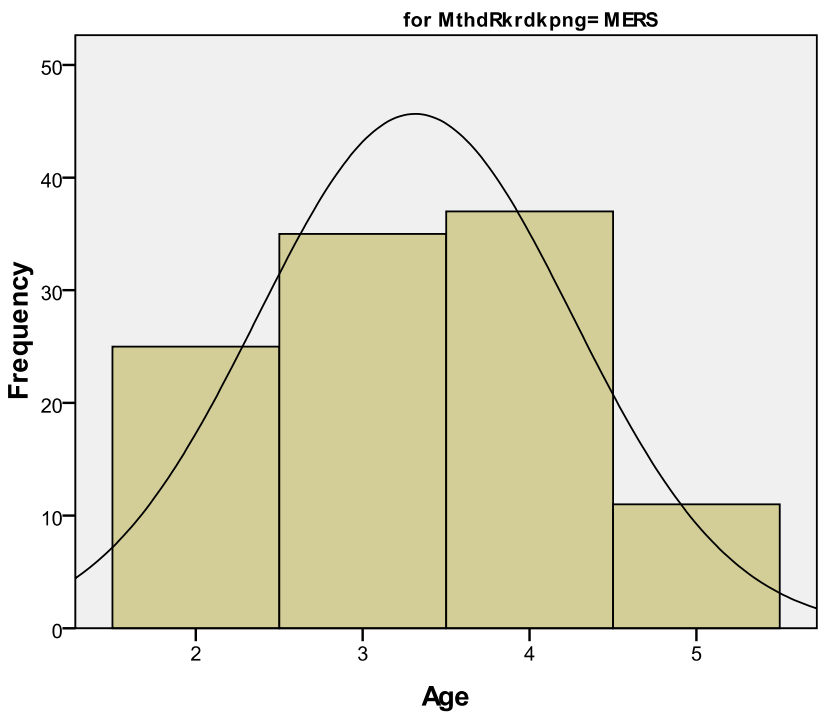

Mean $=3.31$ Std. Dev. $=0.944$
$N=108$

Histogram

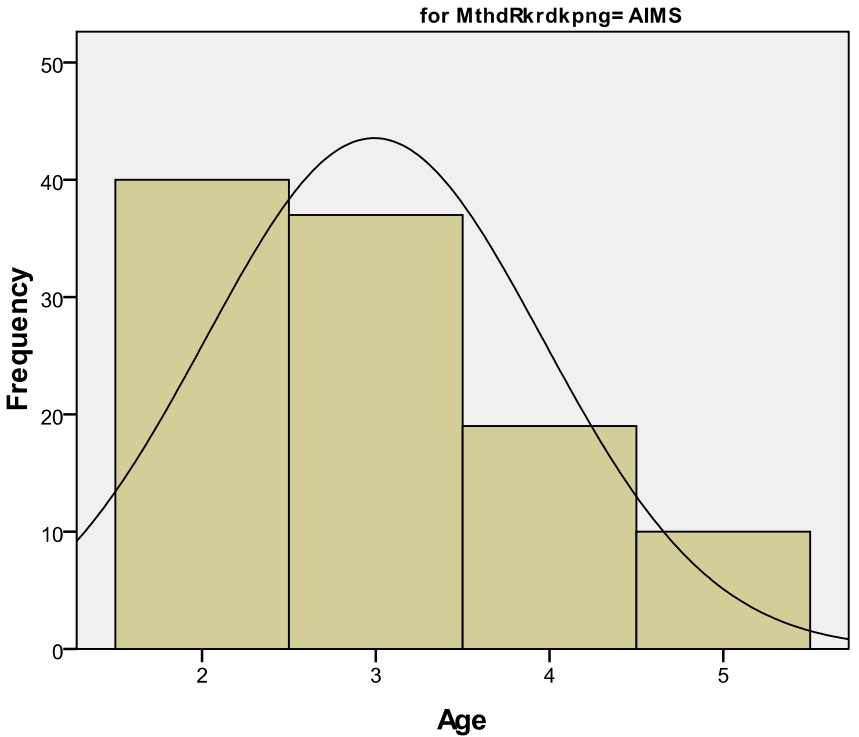

Mean $=2.99$

$\begin{aligned} \text { Std. Dev. } & =0.971 \\ N=106 & \end{aligned}$ 


\section{Histogram}

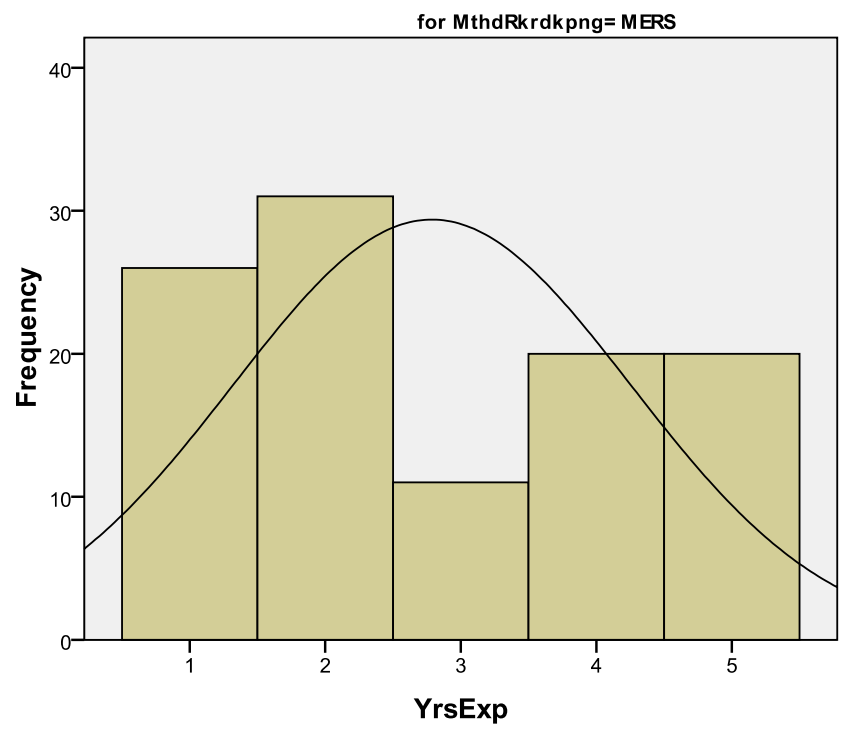

Mean $=2.79$

$\mathrm{Nev}=1.466$
$\mathrm{~N}=108$ 


\section{Histogram}
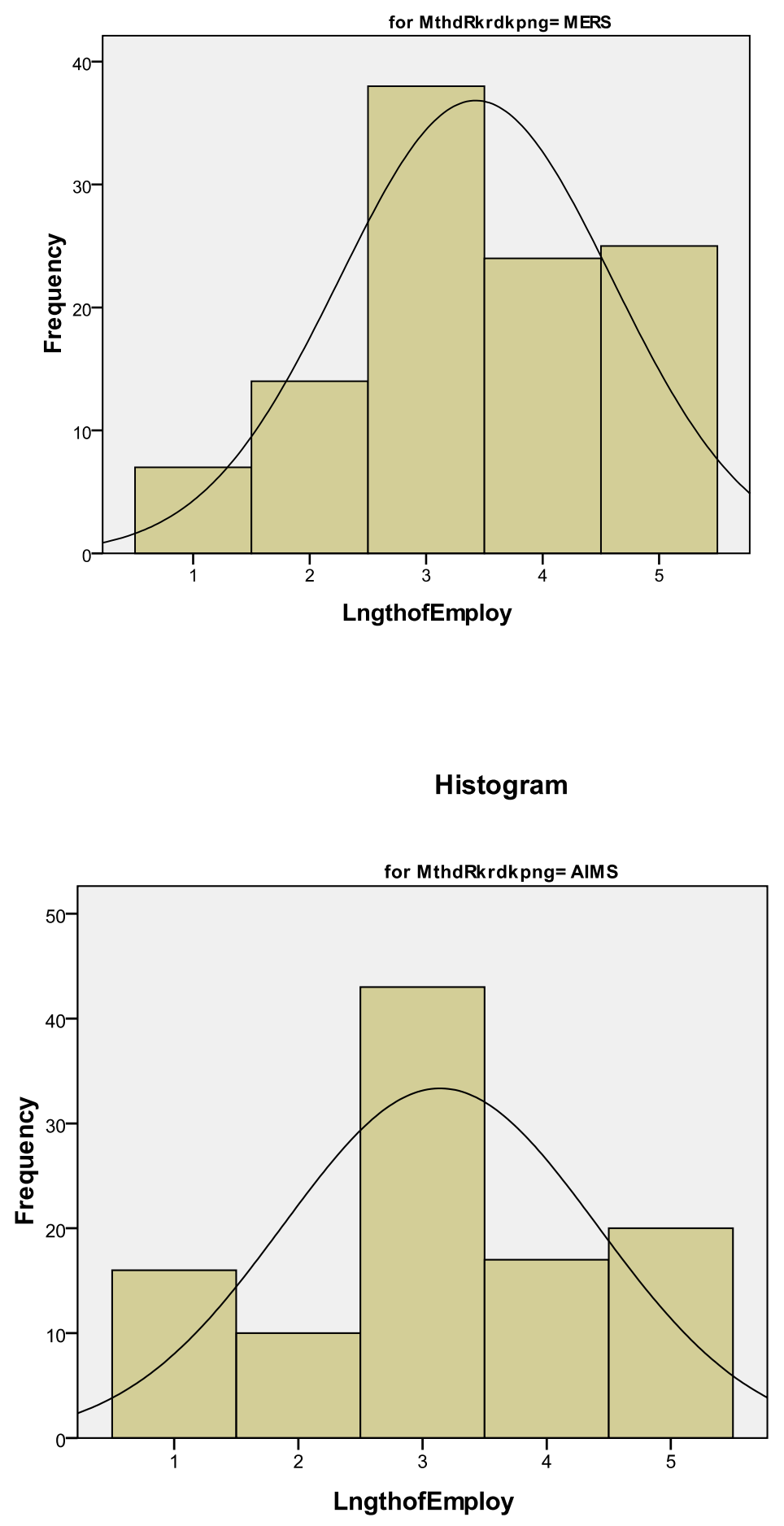

Mean $=3.43$ Std. Dev $=1.17$

$\mathrm{N}=108$

- Normal

\section{Histogram}

Mean $=3.14$ Std. Dev $=1.268$ $\mathrm{N}=106$ 


\section{Histogram}

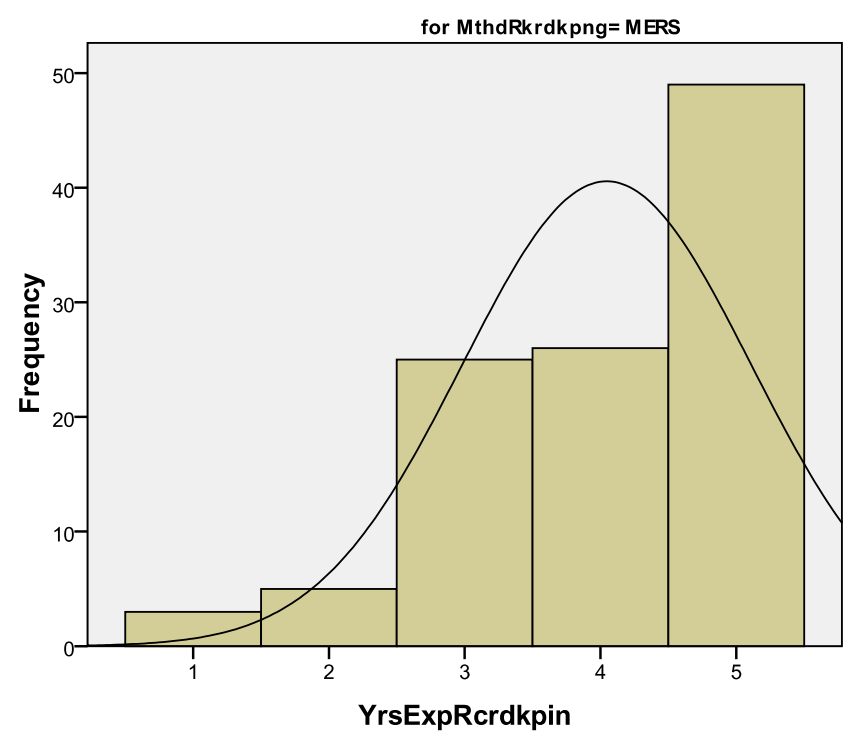

Mean $=4.05$ Std. Dev $=1.062$
$N=108$

- Normal

\section{Histogram}

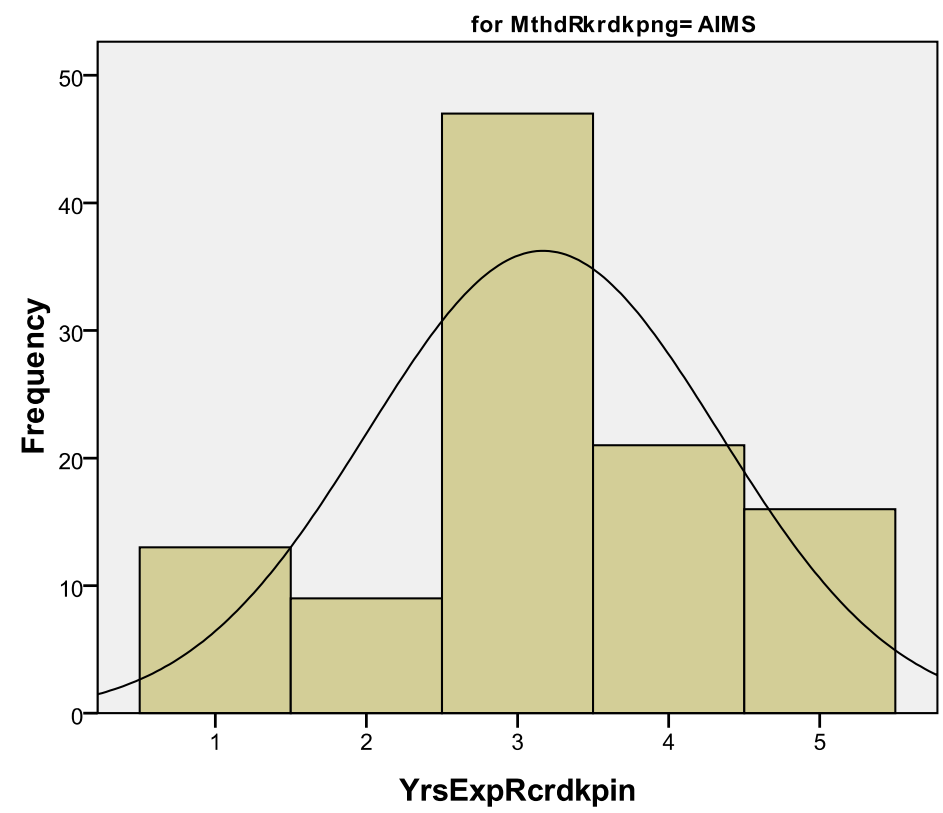

Mean $=3.17$ Std. $\begin{gathered}\mathrm{Dev} \\ \mathrm{N}=106\end{gathered}$ 


\section{Histogram}

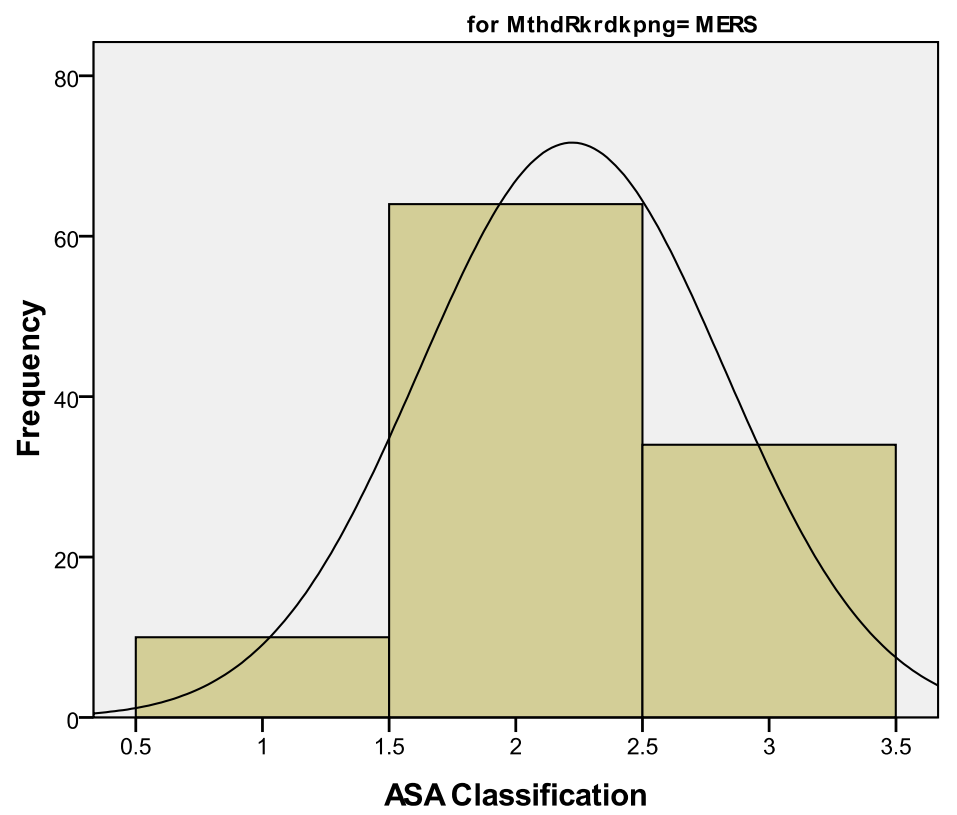

Mean $=2.22$

Std. Dev. $=0.601$

$\mathrm{N}=108$

- Normal

Histogram

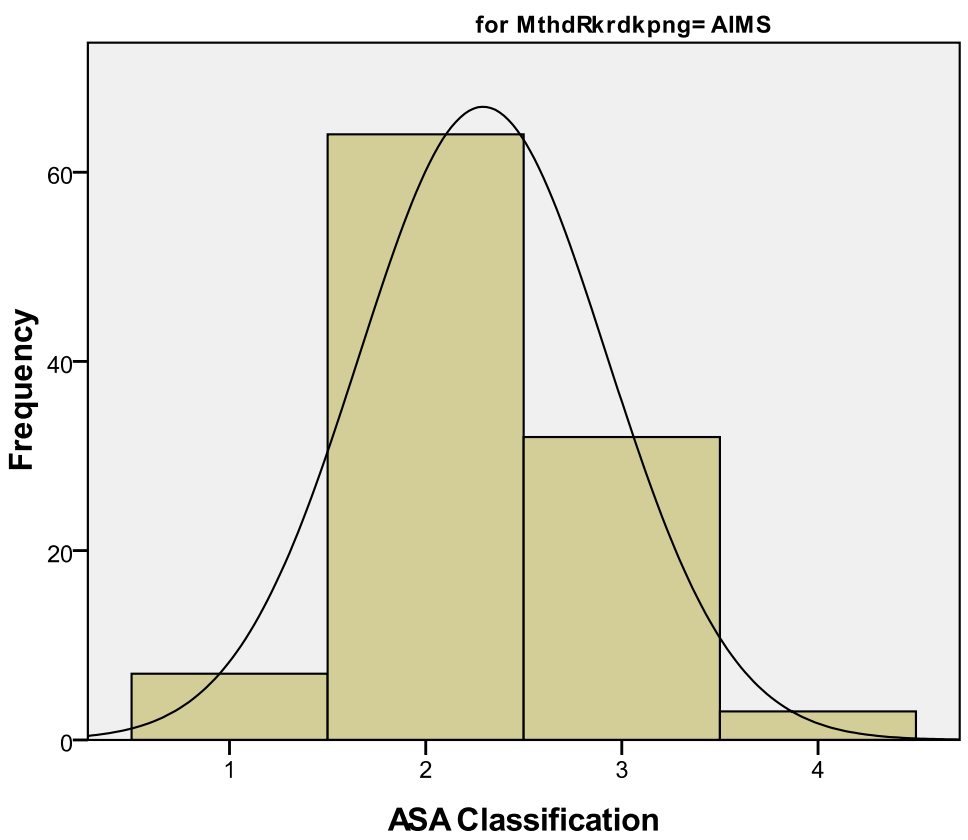

Mean $=2.29$ Std. $\mathrm{Dev}=0.632$
$\mathrm{~N}=106$ 
APPENDIX E: HISTOGRAMS OF DEPENDENT VARIABLES 
Histograms of Dependent Variables

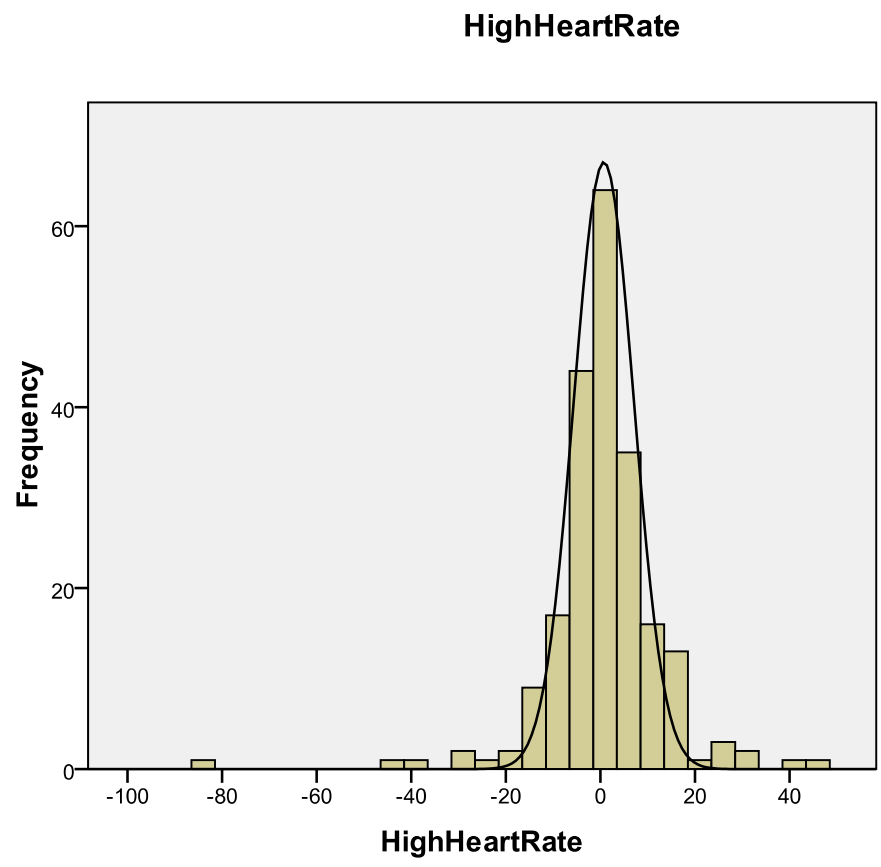
Mean $=0.82$ Std. Dev. $=12.296$

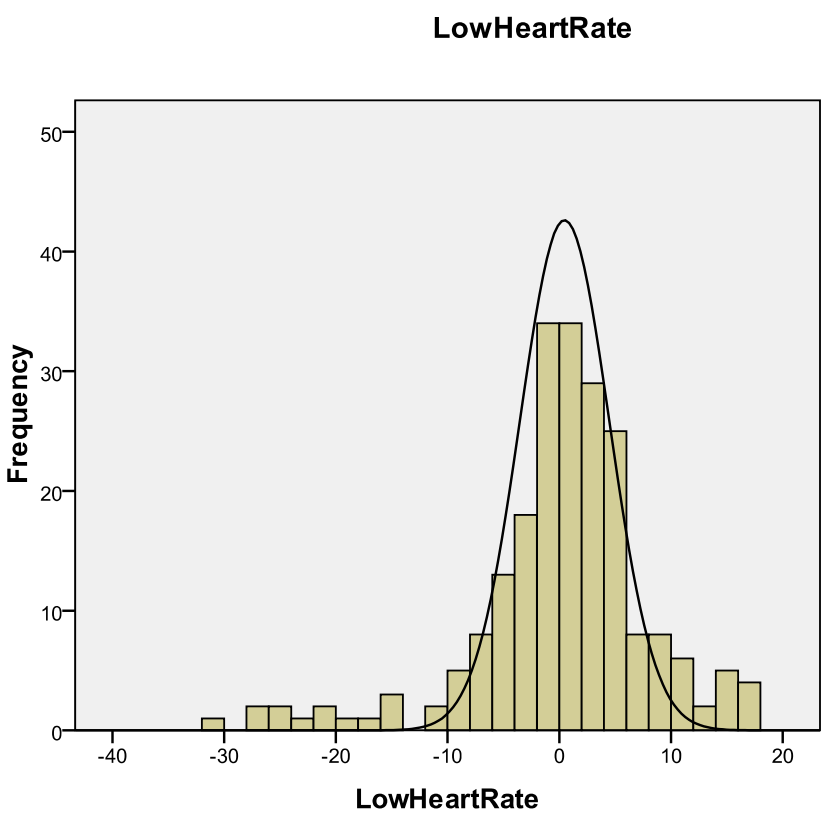

Mean $=-0.29$
Men Std. Dev. $=7.2907$ $\mathrm{N}=2$ 
HighSBP

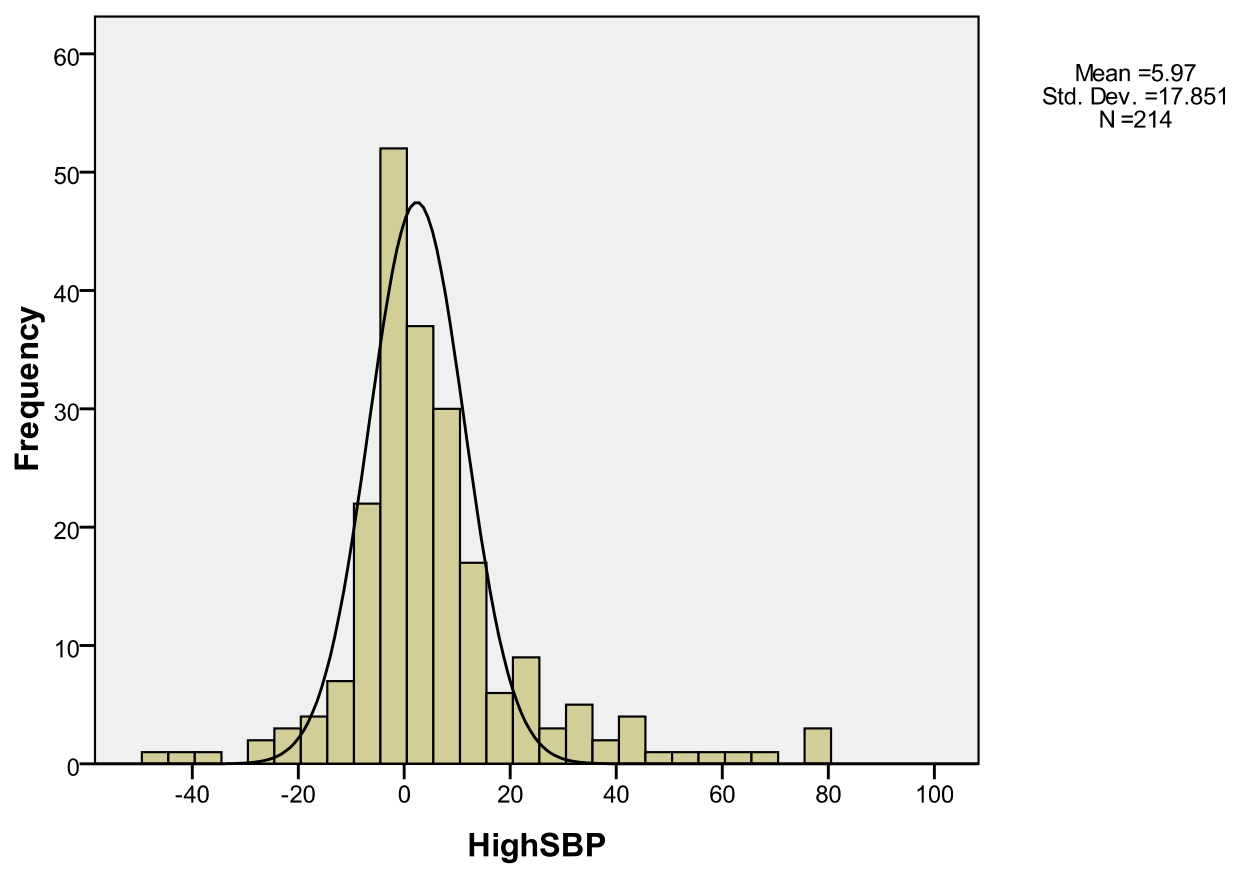

LowSBP

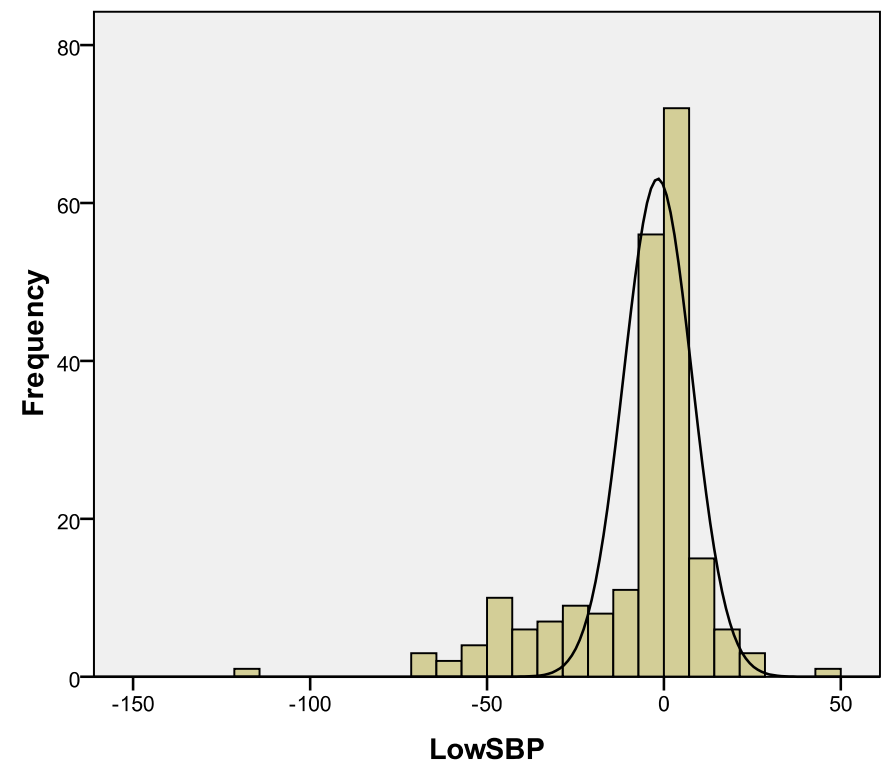

$\begin{aligned} \text { Mean } & =-7.87 \\ \text { Std. } & =20.334\end{aligned}$ $\mathrm{N}=214$ 
HighETCO2

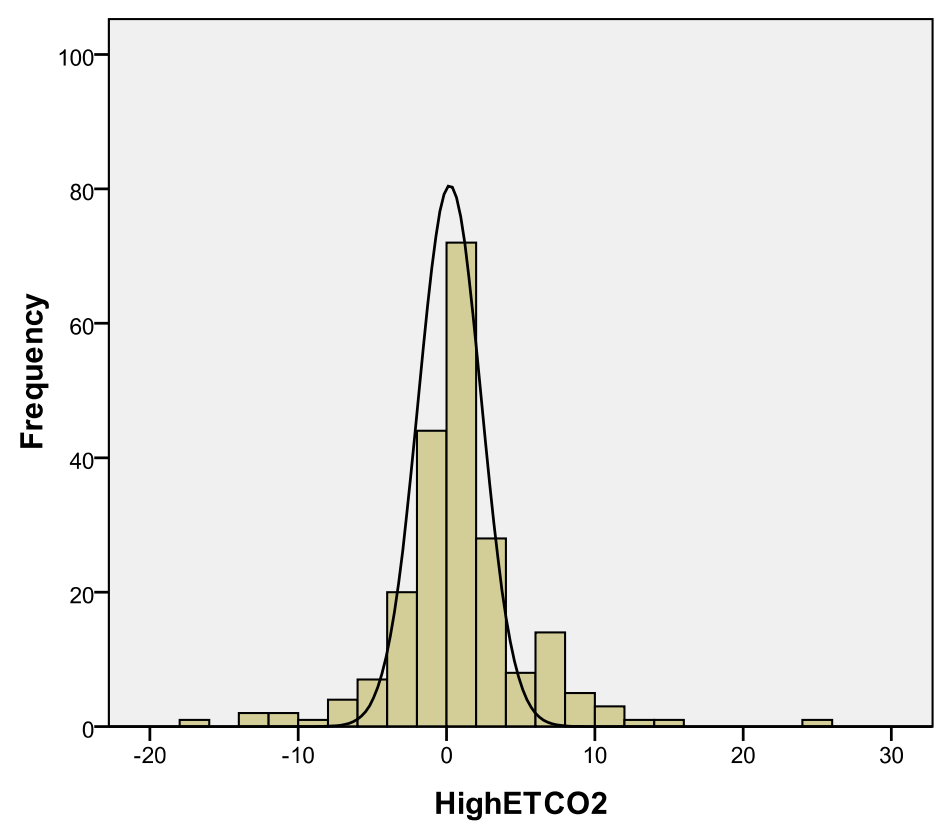

$\begin{aligned} \text { Mean } & =0.34 \\ \text { Std. } & \end{aligned}$ Std. Dev. $=4.523$
$N=214$

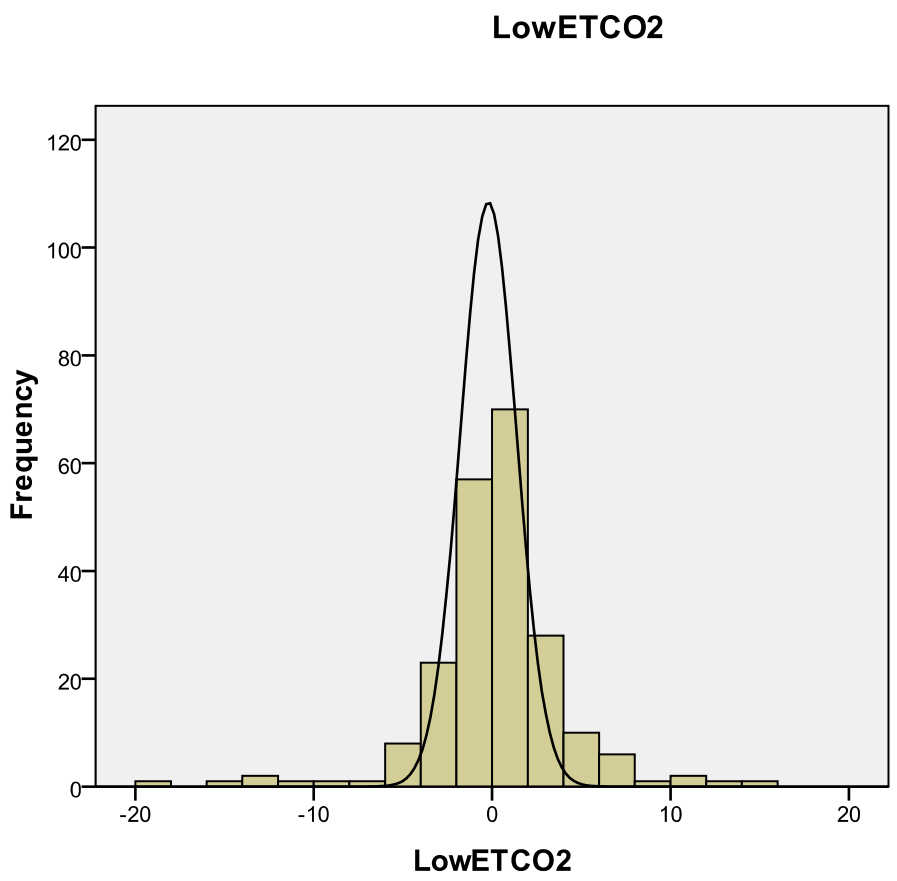

Mean $=-0.23$ Std. Dev. $=3.841$ 


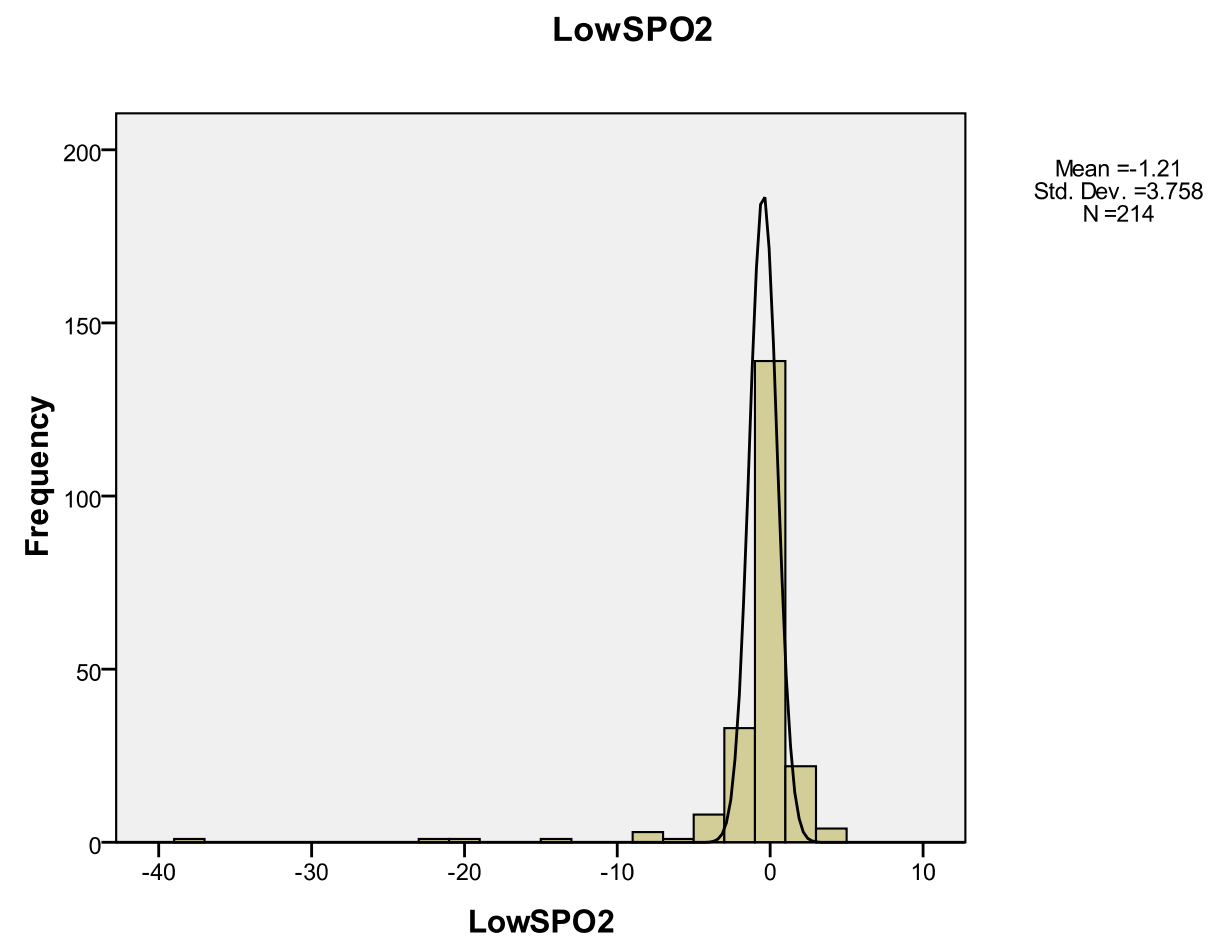


HighlnspPress

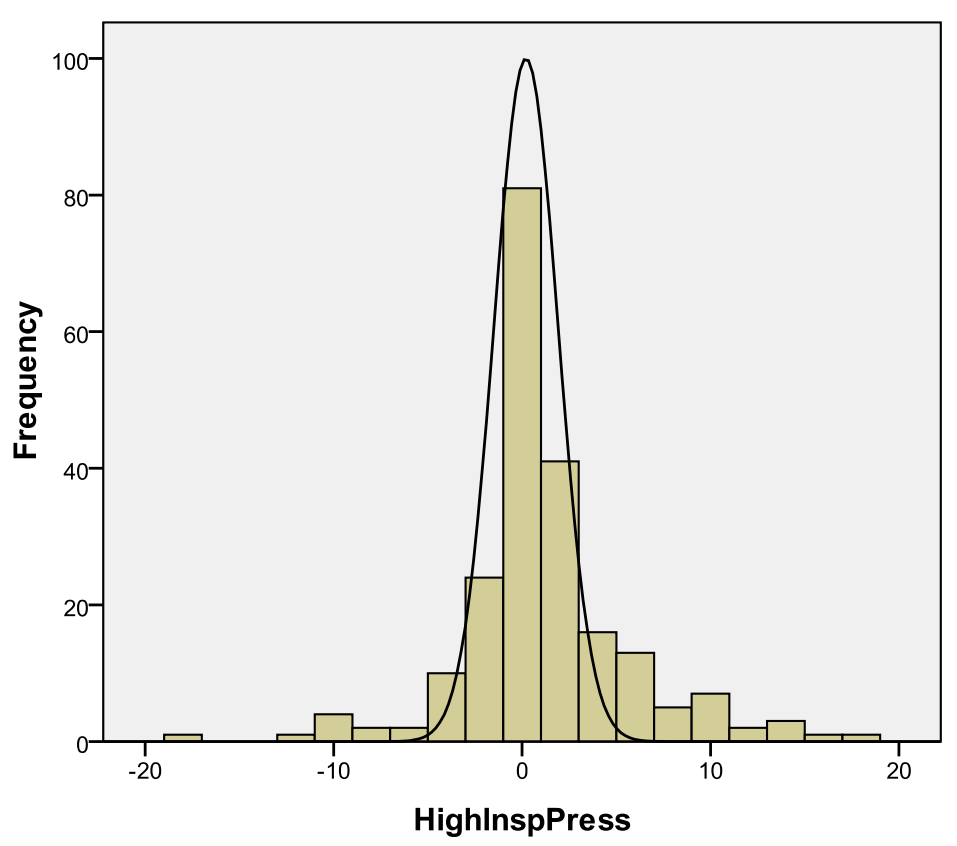

Mean $=0.77$

Std. Dev. $=4.512$
$N=214$

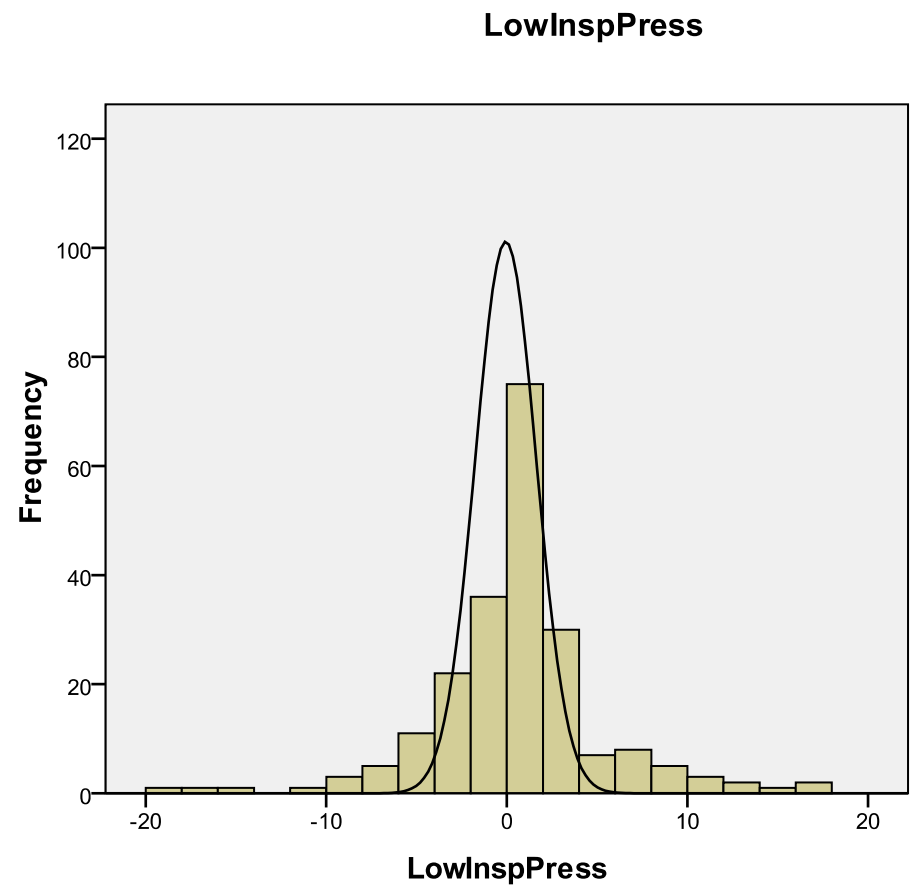

Mean $=0$

Std. Dev. $=4.659$

$\mathrm{N}=214$ 


\section{TotalFluidVol}

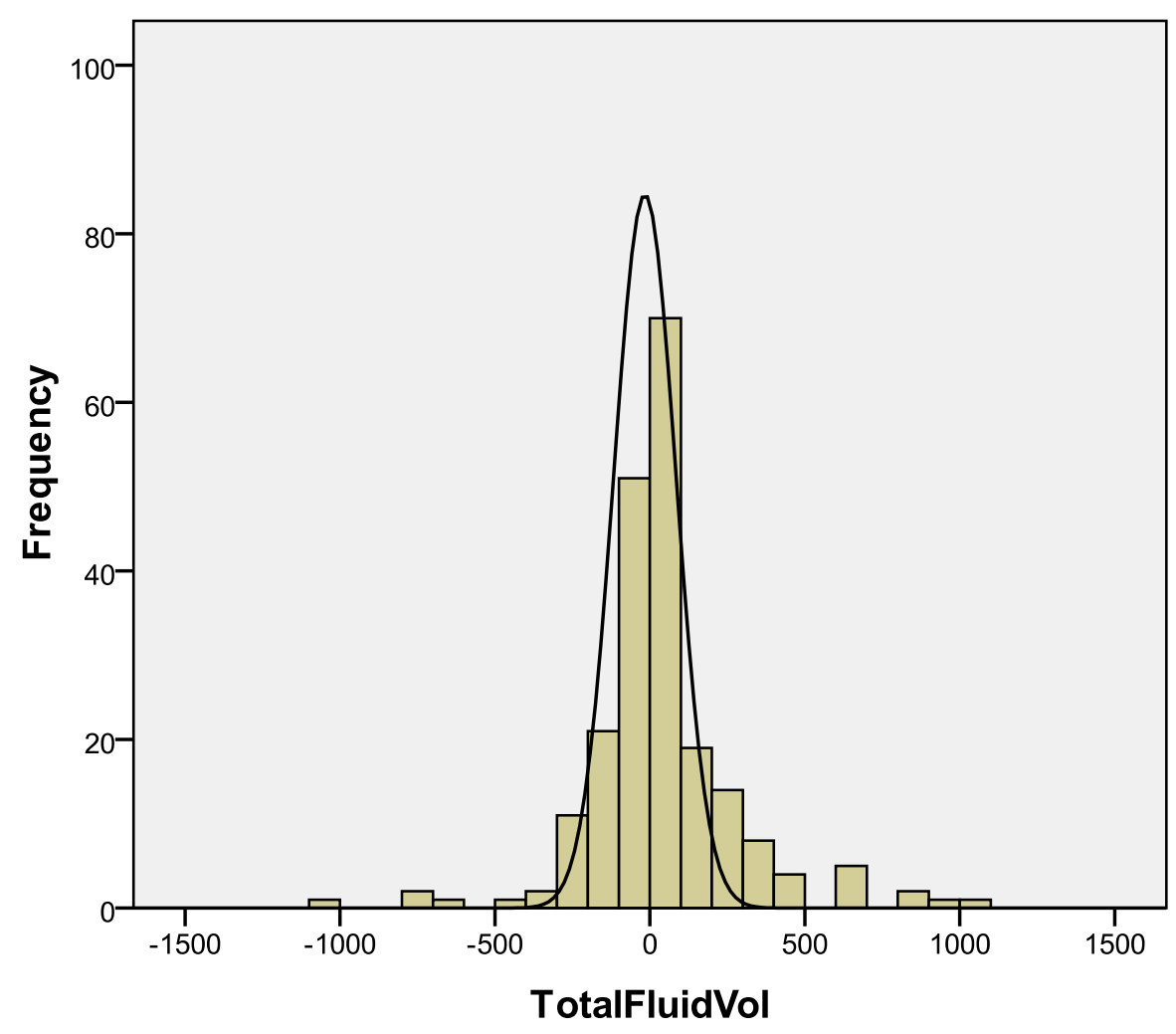

$\begin{aligned} \text { Mean } & =-0.02 \\ \text { Std. Dev. } & =241.193\end{aligned}$

$\mathrm{N}=214$ 
APPENDIX F: DATA ANALYSIS RESULTS - RESEARCH QUESTION \#2 


\section{Research Question \#2}

Dependent Variable - High Heart Rate

$\underline{\text { Tests A-D - High Heart Rate }}$

Descriptive Statistics - Tests A-D

\begin{tabular}{lccc}
\hline & Mean & Standard Deviation & $\mathrm{N}$ \\
\hline O2 & -2.05 & 23.561 & 20 \\
O5 & 1.90 & 14.739 & 20 \\
O7 & 6.65 & 8.331 & 20 \\
O9 & .70 & 8.228 & 20 \\
\hline
\end{tabular}

Main \& Interaction Effects - Tests A-D

\begin{tabular}{|c|c|c|c|c|c|c|c|}
\hline \multicolumn{2}{|c|}{ Source } & $\begin{array}{l}\text { Type III Sum } \\
\text { of Squares }\end{array}$ & $\mathrm{df}$ & $\begin{array}{l}\text { Mean } \\
\text { Square }\end{array}$ & $\mathrm{F}$ & Significance & $\begin{array}{c}\text { Partial } \\
\text { Eta } \\
\text { Squared }\end{array}$ \\
\hline \multicolumn{2}{|l|}{ Pretest } & 281.250 & 1 & 281.250 & 1.287 & .272 & .063 \\
\hline \multicolumn{2}{|l|}{ Treatment } & 20 & 1 & 20 & .108 & .746 & .061 \\
\hline \multicolumn{2}{|c|}{ Pretest*Treatment } & 490.050 & 1 & 490.050 & 2.321 & .144 & .109 \\
\hline Test & Effect & & put & $\mathrm{F}$ & & Significance & $\begin{array}{l}\text { Partial Eta } \\
\text { Squared }\end{array}$ \\
\hline Test A & Interaction & Pretest*' & reatment & at 2.321 & & 144 & .109 \\
\hline Test B & Main & Treatme & & .108 & & 746 & .061 \\
\hline Test $\mathrm{C}$ & Main & Pretest & & 1.287 & & 272 & .063 \\
\hline Test D & Main & Treatme & & .108 & & 746 & .061 \\
\hline
\end{tabular}


$\underline{\text { Test E - High Heart Rate }}$

Descriptive Statistics - Test E

\begin{tabular}{cccc}
\hline & Mean & Standard Deviation & $\mathrm{N}$ \\
\hline $\mathrm{O} 2$ & -2.05 & 23.561 & 20 \\
$\mathrm{O} 5$ & 1.90 & 14.739 & 20 \\
\hline
\end{tabular}

Main \& Interaction Effects - Test E

\begin{tabular}{lcccccc}
\hline \multicolumn{1}{c}{ Source } & $\begin{array}{c}\text { Type III Sum of } \\
\text { Squares }\end{array}$ & df & $\begin{array}{c}\text { Mean } \\
\text { Square }\end{array}$ & F & Significance & $\begin{array}{c}\text { Partial } \\
\text { Eta } \\
\text { Squared }\end{array}$ \\
\hline Postest & 122.139 & 1 & 122.139 & .353 & .560 & .020 \\
Posttest*HHRO1 & 33.948 & 1 & 33.948 & .098 & .758 & .006 \\
Posttest*HHRO4 & 174.454 & 1 & 174.454 & .505 & .487 & .029 \\
\hline
\end{tabular}

\begin{tabular}{cccccc}
\hline Test & Effect & Output & F & Significance & $\begin{array}{c}\text { Partial Eta } \\
\text { Squared }\end{array}$ \\
\hline Test E & Interaction & Posttest*HHRO1 & .098 & .758 & .006 \\
Test E & Interaction & Posttest*HHRO4 & .505 & .487 & .029 \\
\hline
\end{tabular}

$\underline{\text { Test F - High Heart Rate }}$

Descriptive Statistics - Test F

\begin{tabular}{cccc}
\hline & Mean & Standard Deviation & $\mathrm{N}$ \\
\hline O1 & -.57 & 13.902 & 21 \\
O9 & 1.86 & 9.614 & 21 \\
O4 & 1.00 & 13.327 & 21 \\
O7 & 6.57 & 8.128 & 21 \\
\hline
\end{tabular}


Main \& Interaction Effects - Test F

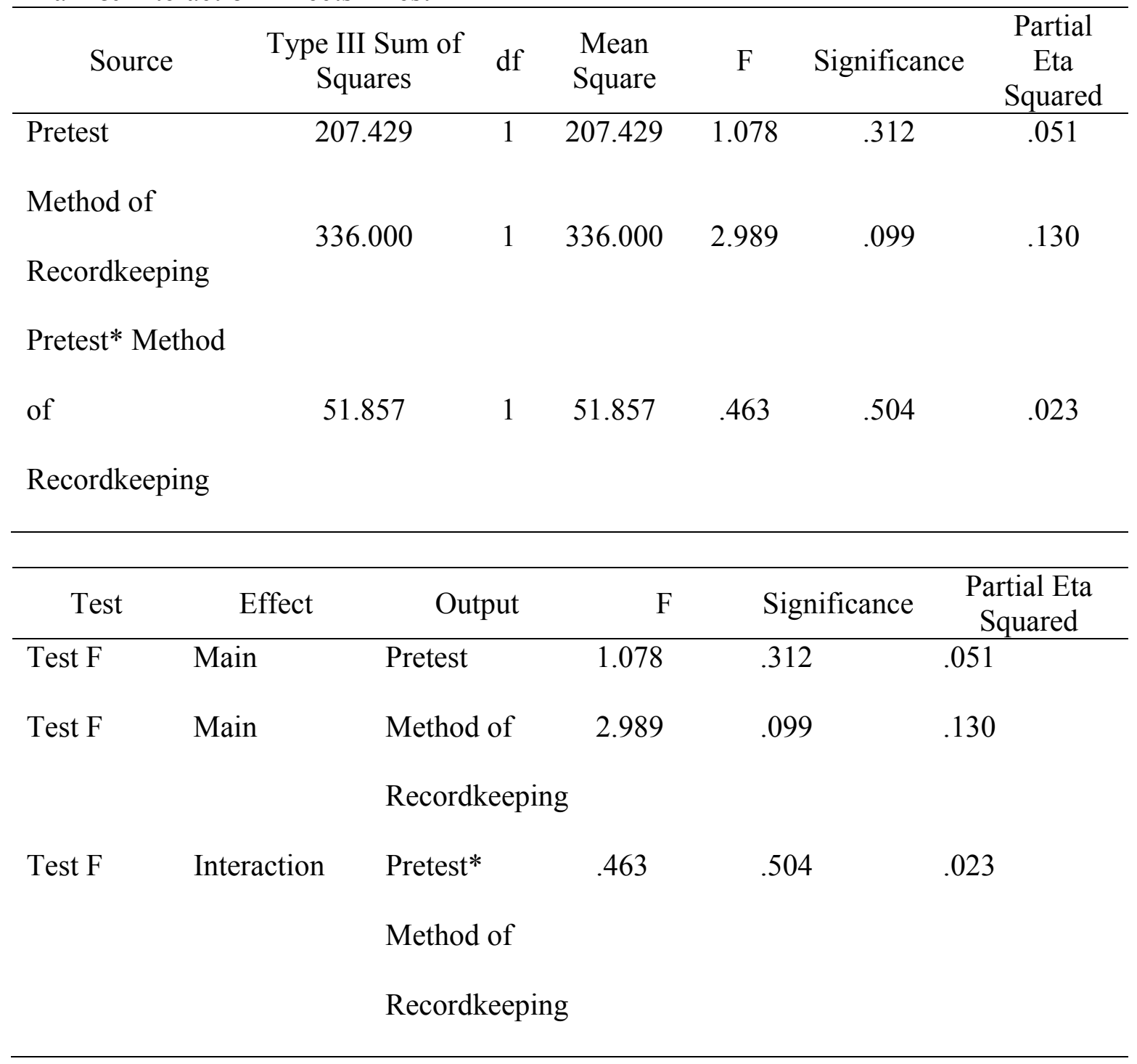

\section{Test G - High Heart Rate}

Descriptive Statistics - Test G

\begin{tabular}{lccc}
\hline & Mean & Standard Deviation & N \\
\hline O1 2 & -.90 & 14.179 & 20 \\
O4 & -2.05 & 23.561 & 20 \\
O5 & 1.00 & 13.673 & 20 \\
& 1.90 & 14.739 & 20 \\
\hline
\end{tabular}


Main \& Interaction Effects - Test G

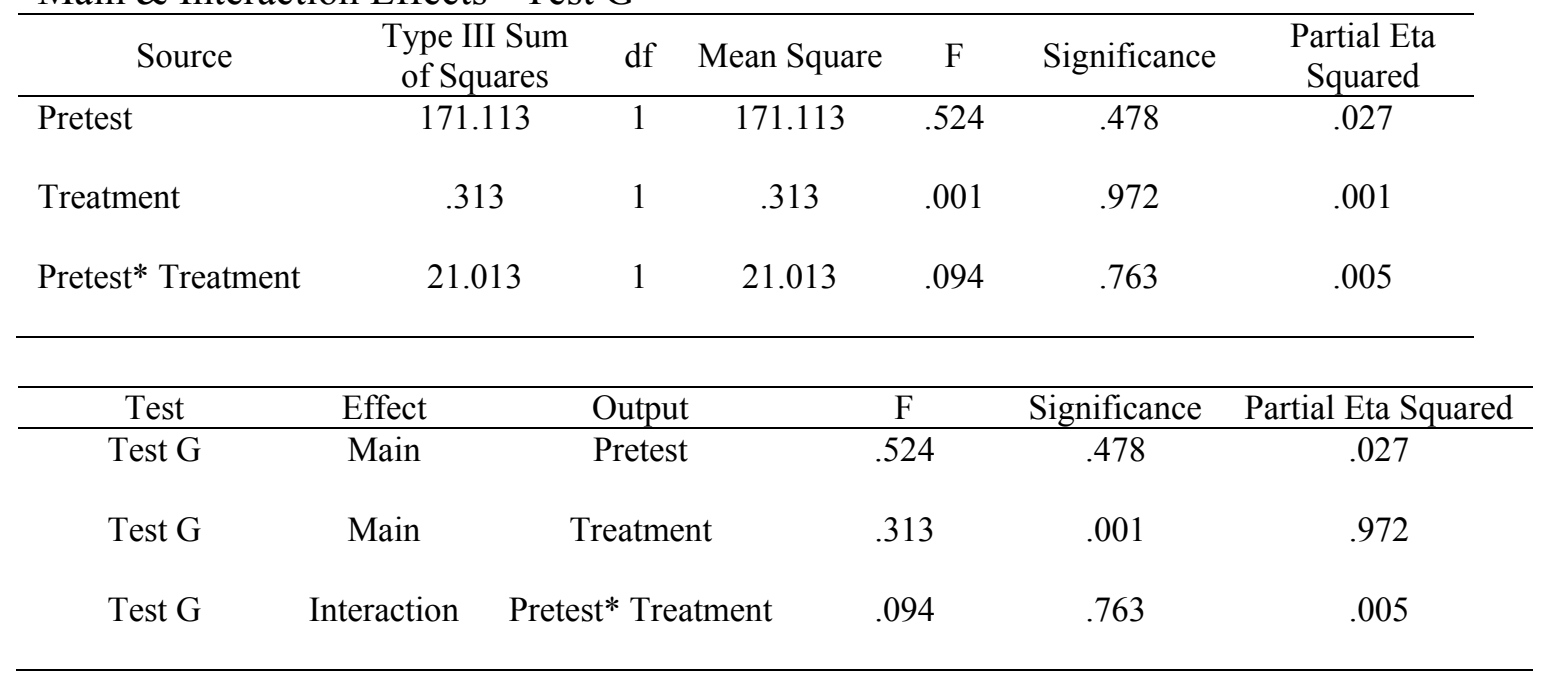




\section{Dependent Variable - Low Heart Rate}

\section{Tests A-D - Low Heart Rate}

Descriptive Statistics - Tests A-D

\begin{tabular}{lccc}
\hline & Mean & Standard Deviation & $\mathrm{N}$ \\
\hline O2 & 2.00 & 8.039 & 20 \\
O5 & -2.20 & 7.925 & 20 \\
O7 & 1.50 & 5.960 & 20 \\
O9 & -1.30 & 6.666 & 20 \\
\hline
\end{tabular}

Main \& Interaction Effects - Tests A-D

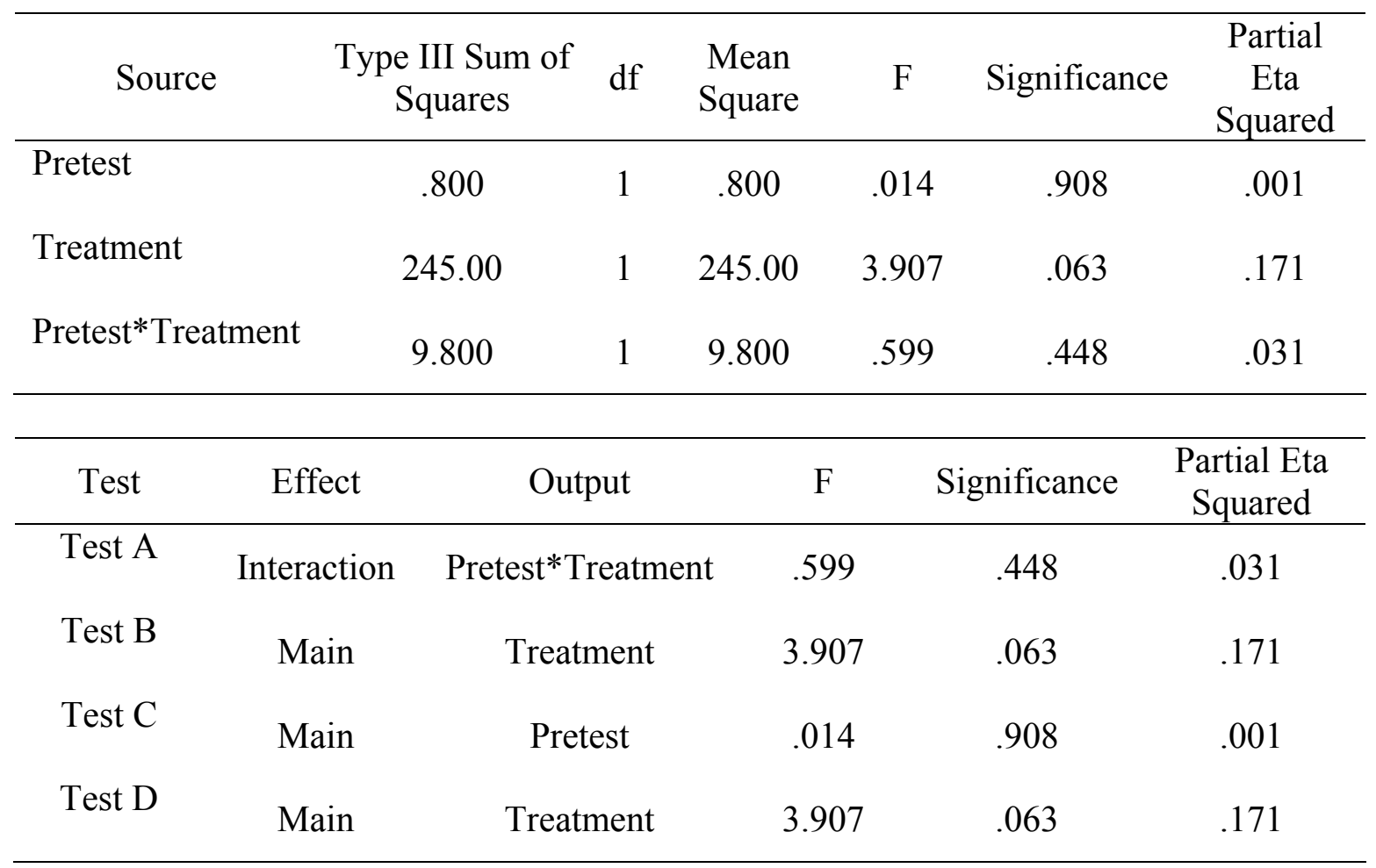


$\underline{\text { Test E - Low Heart Rate }}$

Descriptive Statistics - Test E

\begin{tabular}{cccc}
\hline & Mean & Standard Deviation & $\mathrm{N}$ \\
\hline O2 & 2.00 & 8.039 & 20 \\
O5 & -2.20 & 7.295 & 20 \\
\hline
\end{tabular}

Main \& Interaction Effects - Test E

\begin{tabular}{lcccccc}
\hline \multicolumn{1}{c}{ Source } & $\begin{array}{c}\text { Type III Sum of } \\
\text { Squares }\end{array}$ & df & $\begin{array}{c}\text { Mean } \\
\text { Square }\end{array}$ & F & Significance & $\begin{array}{c}\text { Partial } \\
\text { Squared }\end{array}$ \\
\hline Postest & 147.935 & 1 & 147.935 & 2.551 & .129 & .130 \\
Posttest*LHRO1 & 2.376 & 1 & 2.376 & .041 & .842 & .002 \\
Posttest*LHRO4 & 27.304 & 1 & 27.304 & .471 & .502 & .027 \\
& & & & & &
\end{tabular}

\begin{tabular}{cccccc}
\hline Test & Effect & Output & F & Significance & $\begin{array}{c}\text { Partial Eta } \\
\text { Squared }\end{array}$ \\
\hline Test E & Interaction & Posttest*LHRO1 & .041 & .842 & .002 \\
Test E & Interaction & Posttest*LHRO4 & .471 & .502 & .027 \\
\hline
\end{tabular}

$\underline{\text { Test F - Low Heart Rate }}$

Descriptive Statistics - Test F

\begin{tabular}{cccc}
\hline O1 & Mean & Standard Deviation & N \\
O9 & -.76 & 10.246 & 21 \\
O4 & -1.33 & 6.499 & 21 \\
O7 & -.86 & 9.150 & 21 \\
& 1.62 & 5.835 & 21 \\
\hline
\end{tabular}


Main \& Interaction Effects - Test F

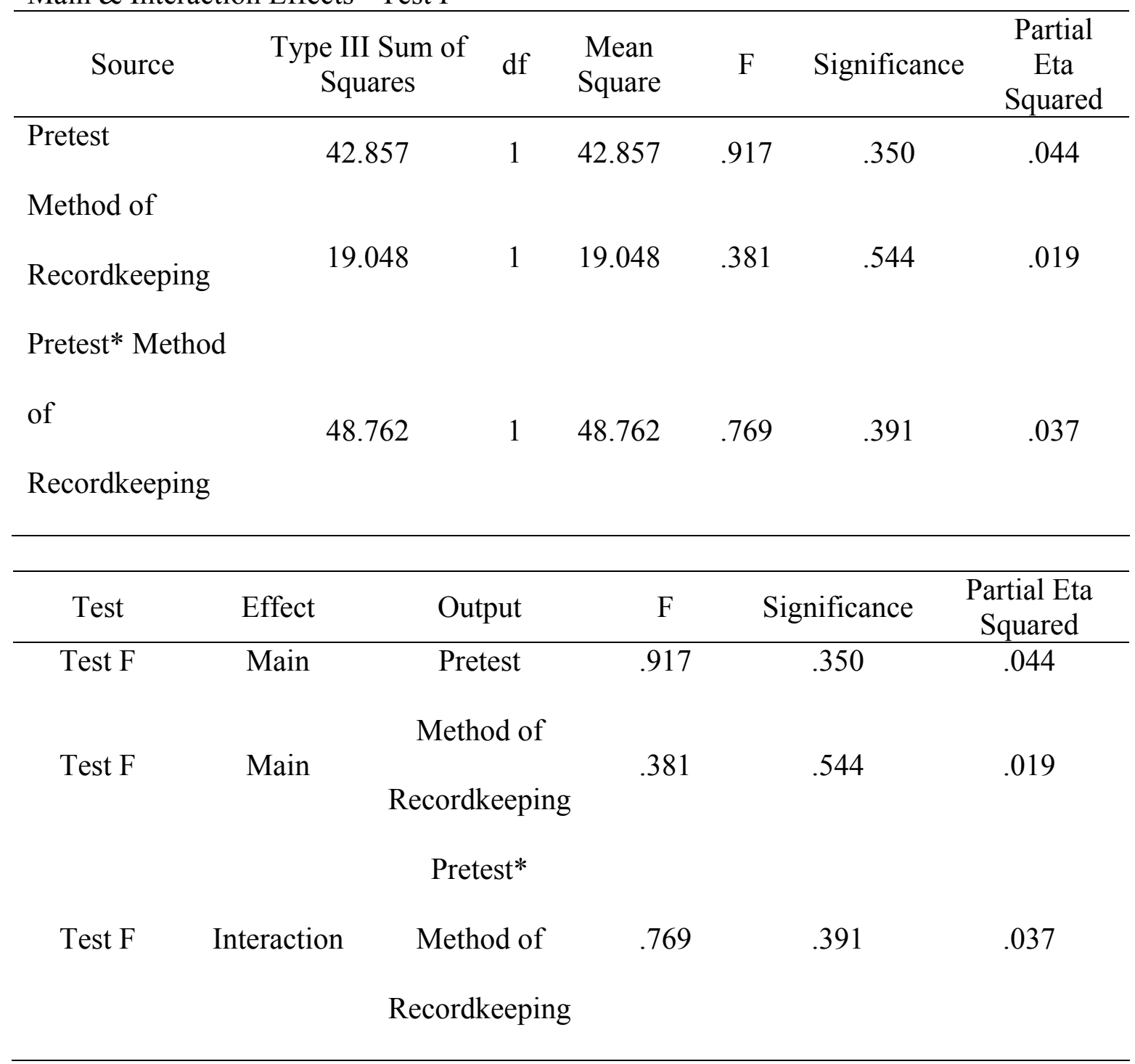

$\underline{\text { Test G - Low Heart Rate }}$

Descriptive Statistics - Test G

\begin{tabular}{cccc}
\hline O1 & Mean & Standard Deviation & $\mathrm{N}$ \\
\hline $\mathrm{O} 2$ & -1.10 & 10.392 & 20 \\
$\mathrm{O} 4$ & 2.00 & 8.039 & 20 \\
$\mathrm{O} 5$ & -1.30 & 9.154 & 20 \\
& -2.20 & 7.295 & 20 \\
\hline
\end{tabular}


Main \& Interaction Effects - Test G

\begin{tabular}{|c|c|c|c|c|c|c|c|}
\hline \multicolumn{2}{|l|}{ Source } & $\begin{array}{c}\text { Type III Sum of } \\
\text { Squares }\end{array}$ & $\mathrm{df}$ & Mean Square & $\mathrm{F}$ & Significance & $\begin{array}{l}\text { Partial Eta } \\
\text { Squared }\end{array}$ \\
\hline Pretest & & 800 & 1 & 96.800 & 1.265 & .275 & .062 \\
\hline Treatment & & 200 & 1 & 24.200 & .280 & .603 & .015 \\
\hline Pretest* Treatmer & & 000 & 1 & 80.000 & 1.194 & 288 & .059 \\
\hline Test & Effect & & & $\mathrm{F}$ & Sign & Partic & Eta Squared \\
\hline Test G & Main & & & 1.265 & & & .062 \\
\hline Test G & Main & Trea & ment & .280 & & & .015 \\
\hline Test $\mathrm{G}$ & Interaction & Pretest* & reatmen & 1.194 & & & .059 \\
\hline
\end{tabular}


Dependent Variable - High Systolic Blood Pressure

Tests A-D - High Systolic Blood Pressure

Descriptive Statistics - Tests A-D

\begin{tabular}{cccc}
\hline & Mean & Standard Deviation & N \\
\hline O2 & 10.95 & 22.814 & 20 \\
O7 & 4.70 & 13.944 & 20 \\
O9 & 12.70 & 20.327 & 20 \\
& 6.70 & 20.901 & 20 \\
\hline
\end{tabular}

Main \& Interaction Effects - Tests A-D

\begin{tabular}{|c|c|c|c|c|c|c|c|c|}
\hline \multicolumn{2}{|c|}{ Source } & $\begin{array}{l}\text { Type III Sum } \\
\text { of Squares }\end{array}$ & $\mathrm{df}$ & \multicolumn{2}{|c|}{$\begin{array}{l}\text { Mean } \\
\text { Square }\end{array}$} & $\mathrm{F}$ & Significance & $\begin{array}{c}\text { Partial } \\
\text { Eta } \\
\text { Squared }\end{array}$ \\
\hline Pretest & & 70.313 & 1 & \multicolumn{2}{|c|}{70.313} & .192 & .666 & .010 \\
\hline Treatment & & 750.313 & 1 & \multicolumn{2}{|c|}{750.313} & 1.937 & .180 & .093 \\
\hline \multicolumn{2}{|c|}{ Pretest*Treatment } & .313 & 1 & .31 & & .001 & .980 & .001 \\
\hline Test & Effect & $\mathrm{Ou}$ & put & & $\mathrm{F}$ & & ignificance & $\begin{array}{c}\text { Partial Eta } \\
\text { Squared }\end{array}$ \\
\hline Test A & Interaction & Pretest*' & reatmen & & .001 & & .980 & .001 \\
\hline Test B & Main & Trea & ment & & 1.937 & & .180 & .093 \\
\hline Test C & Main & $\operatorname{Pre}$ & est & & .192 & & .666 & .010 \\
\hline Test D & Main & Trea & ment & & 1.937 & & .180 & .093 \\
\hline
\end{tabular}


$\underline{\text { Test E - High Systolic Blood Pressure }}$

Descriptive Statistics - Test E

\begin{tabular}{cccc}
\hline & Mean & Standard Deviation & $\mathrm{N}$ \\
\hline O2 & 10.95 & 22.814 & 20 \\
O5 & 4.70 & 13.944 & 20 \\
\hline
\end{tabular}

Main \& Interaction Effects - Test E

\begin{tabular}{lcccccc}
\hline \multicolumn{1}{c}{ Source } & $\begin{array}{c}\text { Type III Sum } \\
\text { of Squares }\end{array}$ & df & $\begin{array}{c}\text { Mean } \\
\text { Square }\end{array}$ & F & Significance & $\begin{array}{c}\text { Partial } \\
\text { Eta } \\
\text { Squared }\end{array}$ \\
\hline Postest & 675.734 & 1 & 675.734 & 2.137 & .162 & .112 \\
Posttest*HSBPO1 & 311.774 & 1 & 311.774 & .986 & .335 & .055 \\
Posttest*HSBPO4 & 1828.297 & 1 & 1828.297 & 5.782 & .028 & .254 \\
\hline
\end{tabular}

\begin{tabular}{cccccc}
\hline Test & Effect & Output & F & Significance & $\begin{array}{c}\text { Partial Eta } \\
\text { Squared }\end{array}$ \\
\hline Test E & Interaction & Posttest*HSBPO1 & .986 & .335 & .055 \\
Test E & Interaction & Posttest*HSBPO4 & 5.782 & .028 & .254 \\
\hline
\end{tabular}

Test F - High Systolic Blood Pressure

Descriptive Statistics - Test F

\begin{tabular}{lccc}
\hline & Mean & Standard Deviation & $\mathrm{N}$ \\
\hline O1 & 5.19 & 11.492 & 21 \\
O9 & 6.52 & 20.388 & 21 \\
O4 & 9.62 & 21.477 & 21 \\
O7 & 12.00 & 20.070 & 21 \\
\hline
\end{tabular}


Main \& Interaction Effects - Test F

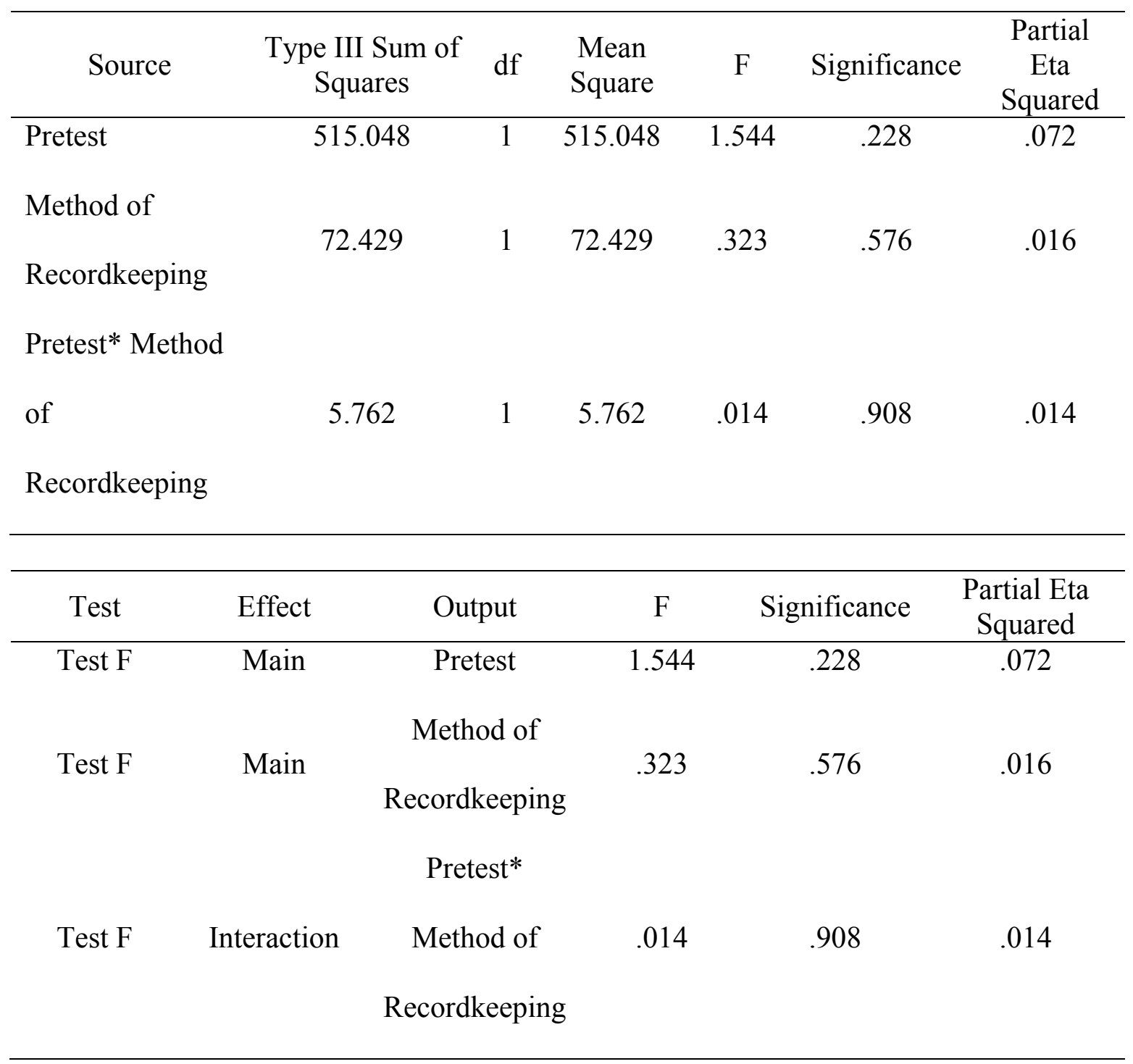


$\underline{\text { Test G - High Systolic Blood Pressure }}$

Descriptive Statistics - Test G

\begin{tabular}{cccc}
\hline & Mean & Standard Deviation & $\mathrm{N}$ \\
\hline O1 & 5.70 & 11.544 & 20 \\
O4 & 10.95 & 22.814 & 20 \\
O5 & 10.40 & 21.727 & 20 \\
& 4.70 & 13.944 & 20 \\
\hline
\end{tabular}

Main \& Interaction Effects - Test G

\begin{tabular}{lcccccc}
\hline \multicolumn{1}{c}{ Source } & $\begin{array}{c}\text { Type III Sum } \\
\text { of Squares }\end{array}$ & df & $\begin{array}{c}\text { Mean } \\
\text { Square }\end{array}$ & F & Significance & $\begin{array}{c}\text { Partial Eta } \\
\text { Squared }\end{array}$ \\
\hline Pretest & 12.012 & 1 & 12.012 & .023 & .882 & .001 \\
Treatment & 1.012 & 1 & 1.012 & .003 & .954 & .000 \\
Pretest* Treatment & 599.513 & 1 & 599.513 & 3.626 & .072 & .160 \\
\hline
\end{tabular}

\begin{tabular}{cccccc}
\hline Test & Effect & Output & F & Significance & Partial Eta Squared \\
\hline Test G & Main & Pretest & .023 & .882 & .001 \\
Test G & Main & Treatment & .003 & .954 & .000 \\
Test G & Interaction & Pretest* Treatment & 3.626 & .072 & .160 \\
\hline
\end{tabular}




\section{Dependent Variable - Low Systolic Blood Pressure}

Tests A-D - Low Systolic Blood Pressure

Descriptive Statistics - Tests A-D

\begin{tabular}{lccc}
\hline & Mean & Standard Deviation & $\mathrm{N}$ \\
\hline O2 & -2.90 & 12.226 & 20 \\
O5 & -7.95 & 21.722 & 20 \\
O7 & -14.90 & 24.999 & 20 \\
O9 & -8.80 & 19.116 & 20 \\
\hline
\end{tabular}

Main \& Interaction Effects - Tests A-D

\begin{tabular}{|c|c|c|c|c|c|c|c|}
\hline \multicolumn{2}{|c|}{ Source } & $\begin{array}{l}\text { Type III Sum } \\
\text { of Squares }\end{array}$ & $\mathrm{df}$ & $\begin{array}{l}\text { Mean } \\
\text { Square }\end{array}$ & $\mathrm{F}$ & Significance & $\begin{array}{c}\text { Partial } \\
\text { Eta } \\
\text { Squared }\end{array}$ \\
\hline \multicolumn{2}{|l|}{ Pretest } & 825.613 & 1 & 825.613 & 1.330 & .263 & .065 \\
\hline \multicolumn{2}{|l|}{ Treatment } & 5.512 & 1 & 5.512 & . 022 & .883 & .001 \\
\hline \multicolumn{2}{|c|}{ Pretest*Treatment } & 621.613 & 1 & 621.613 & 1.627 & .217 & .079 \\
\hline Test & Effect & & & & & Significance & $\begin{array}{c}\text { Partial Eta } \\
\text { Squared }\end{array}$ \\
\hline Test A & Interaction & Pretest* & reatmen & & & .217 & .079 \\
\hline Test B & Main & Tre & ment & & & .883 & .001 \\
\hline Test C & Main & & & & & .263 & .065 \\
\hline Test D & Main & Tre & ment & & 22 & .883 & .001 \\
\hline
\end{tabular}


$\underline{\text { Test E - Low Systolic Blood Pressure }}$

Descriptive Statistics - Test E

\begin{tabular}{cccc}
\hline & Mean & Standard Deviation & $\mathrm{N}$ \\
\hline O2 & -2.90 & 12.226 & 20 \\
O5 & -7.95 & 21.722 & 20 \\
\hline
\end{tabular}

Main \& Interaction Effects - Test E

\begin{tabular}{|c|c|c|c|c|c|c|c|c|c|}
\hline \multicolumn{2}{|c|}{ Source } & \multicolumn{2}{|c|}{$\begin{array}{l}\text { Type III Sum } \\
\text { of Squares }\end{array}$} & $\mathrm{df}$ & \multicolumn{2}{|c|}{$\begin{array}{l}\text { Mean } \\
\text { Square }\end{array}$} & $\mathrm{F}$ & Significance & $\begin{array}{l}\text { Partial } \\
\text { Eta } \\
\text { Squared }\end{array}$ \\
\hline \multicolumn{2}{|l|}{ Postest } & \multicolumn{2}{|c|}{894.735} & 1 & \multicolumn{2}{|c|}{894.735} & 4.126 & .058 & .195 \\
\hline \multicolumn{2}{|c|}{ Posttest*LSBPO1 } & \multicolumn{2}{|c|}{1008.862} & 1 & \multicolumn{2}{|c|}{1008.862} & 4.653 & .046 & .215 \\
\hline \multicolumn{2}{|c|}{ Posttest*LSBPO4 } & \multicolumn{2}{|c|}{5.508} & 1 & \multicolumn{2}{|c|}{5.508} & .025 & .875 & .001 \\
\hline Test & & \multicolumn{4}{|c|}{ Output } & $\mathrm{F}$ & \multicolumn{2}{|c|}{ Significance } & $\begin{array}{l}\text { Partial Eta } \\
\text { Squared }\end{array}$ \\
\hline Test $\mathrm{E}$ & \multicolumn{2}{|c|}{ Interaction } & \multicolumn{3}{|c|}{ Posttest*LSBPO1 } & 4.653 & \multicolumn{2}{|r|}{.046} & .215 \\
\hline Test $\mathrm{E}$ & \multicolumn{2}{|c|}{ Interaction } & \multicolumn{3}{|c|}{ Posttest*LSBPO4 } & .025 & & .875 & .001 \\
\hline
\end{tabular}

Test F - Low Systolic Blood Pressure

Descriptive Statistics - Test F

\begin{tabular}{lccc}
\hline & Mean & Standard Deviation & $\mathrm{N}$ \\
\hline O1 & -11.33 & 21.763 & 21 \\
O9 & -8.43 & 18.710 & 21 \\
O4 & -1.62 & 12.714 & 21 \\
O7 & -13.81 & 24.873 & 21 \\
\hline
\end{tabular}


Main \& Interaction Effects - Test F

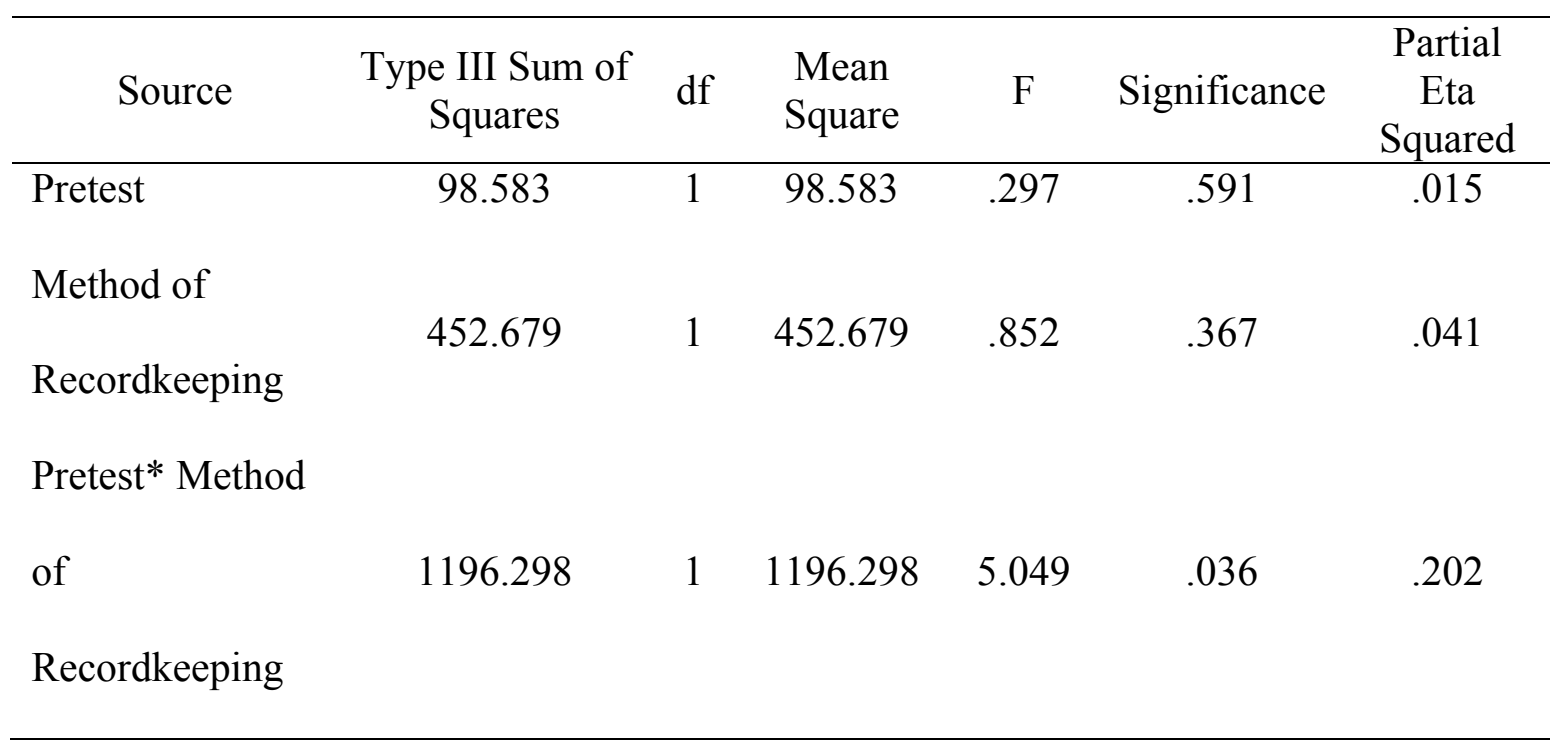

\begin{tabular}{cccccc}
\hline Test & Effect & Output & F & Significance & $\begin{array}{c}\text { Partial Eta } \\
\text { Squared }\end{array}$ \\
\hline Test F & Main & Pretest & .297 & .591 & .015 \\
Test F & Main & Method of & & & \\
& & Recordkeeping & .852 & .367 & .041 \\
& & Pretest* & & \\
Test F & Interaction & Method of & 5.049 & .036 & .202 \\
& & Recordkeeping & & \\
& & &
\end{tabular}


$\underline{\text { Test G - Low Systolic Blood Pressure }}$

Descriptive Statistics - Test G

\begin{tabular}{lccc}
\hline & Mean & Standard Deviation & $\mathrm{N}$ \\
\hline O1 & -11.45 & 22.322 & 20 \\
O2 & -2.90 & 12.226 & 20 \\
O4 & -2.15 & 12.803 & 20 \\
O5 & -7.95 & 21.722 & 20 \\
\hline
\end{tabular}

Main \& Interaction Effects - Test G

\begin{tabular}{|c|c|c|c|c|c|c|c|c|}
\hline Source & \multicolumn{2}{|c|}{$\begin{array}{l}\text { Type III Sum of } \\
\text { Squares }\end{array}$} & $\mathrm{df}$ & \multicolumn{2}{|c|}{$\begin{array}{l}\text { Mean } \\
\text { Square }\end{array}$} & $\mathrm{F}$ & Significance & $\begin{array}{l}\text { Partial Eta } \\
\text { Squared }\end{array}$ \\
\hline Pretest & \multicolumn{2}{|c|}{90.313} & 1 & \multicolumn{2}{|c|}{90.313} & .272 & .608 & .014 \\
\hline Treatment & \multicolumn{2}{|c|}{37.812} & 1 & \multicolumn{2}{|c|}{37.812} & .079 & .782 & .004 \\
\hline Pretest* Treatmen & \multicolumn{2}{|c|}{1029.613} & 1 & \multicolumn{2}{|c|}{1029.613} & 7.550 & .013 & .284 \\
\hline Test & Effect & \multicolumn{2}{|c|}{ Output } & & $\mathrm{F}$ & \multicolumn{2}{|c|}{ Significance } & artial Eta Squared \\
\hline Test G & Main & \multicolumn{2}{|c|}{ Pretest } & \multicolumn{2}{|r|}{.272} & \multicolumn{2}{|c|}{.608} & .014 \\
\hline Test G & Main & \multicolumn{2}{|c|}{ Treatment } & \multicolumn{2}{|r|}{.079} & \multicolumn{2}{|r|}{.782} & .004 \\
\hline Test G & Interaction & \multicolumn{2}{|c|}{ Pretest* Treatmen } & & 7.550 & & .013 & .284 \\
\hline
\end{tabular}




\section{Dependent Variable - High $\mathrm{ETCO}_{2}$}

$\underline{\text { Tests A-D - High } \mathrm{ETCO}_{2}}$

Descriptive Statistics - Tests A-D

\begin{tabular}{lccc}
\hline & Mean & Standard Deviation & $\mathrm{N}$ \\
\hline $\mathrm{O} 2$ & .90 & 5.261 & 20 \\
$\mathrm{O} 5$ & -.65 & 3.924 & 20 \\
$\mathrm{O} 7$ & 2.15 & 6.124 & 20 \\
$\mathrm{O} 9$ & -.65 & 3.675 & 20 \\
\hline
\end{tabular}

Main \& Interaction Effects - Tests A-D

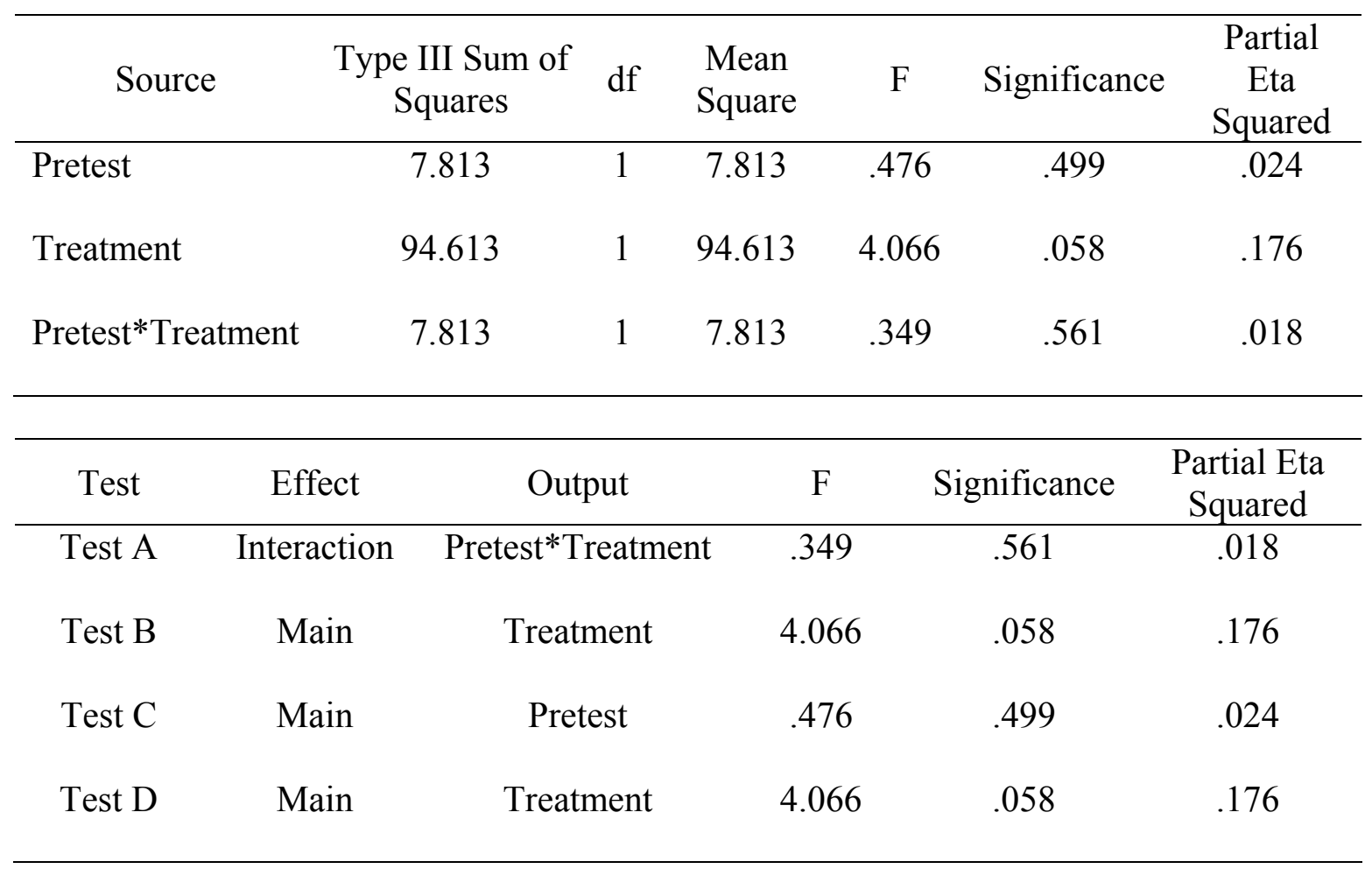


$\underline{\text { Test E - High ETCO }} \underline{2}_{2}$

Descriptive Statistics - Test E

\begin{tabular}{cccc}
\hline & Mean & Standard Deviation & $\mathrm{N}$ \\
\hline $\mathrm{O} 2$ & .90 & 5.261 & 20 \\
$\mathrm{O} 5$ & -.65 & 3.924 & 20 \\
\hline
\end{tabular}

Main \& Interaction Effects - Test E

\begin{tabular}{|c|c|c|c|c|c|c|c|}
\hline \multicolumn{2}{|c|}{ Source } & $\begin{array}{l}\text { Type III Sum of } \\
\text { Squares }\end{array}$ & df & Mean Square & $\mathrm{F}$ & Significance & $\begin{array}{c}\text { Partial Eta } \\
\text { Squared }\end{array}$ \\
\hline \multicolumn{2}{|l|}{ Postest } & 23.820 & 1 & 23.820 & 1.064 & .317 & .059 \\
\hline \multicolumn{2}{|c|}{ Posttest*HETCO ${ }_{2} \mathrm{O} 1$} & 10.726 & 1 & 10.726 & .479 & .498 & .027 \\
\hline \multicolumn{2}{|c|}{ Posttest* $\mathrm{HETCO}_{2} \mathrm{O} 4$} & .634 & 1 & .634 & .028 & .868 & .002 \\
\hline Test & Effect & Outpr & & $\mathrm{F}$ & Signi & icance & $\begin{array}{l}\text { Partial Eta } \\
\text { Squared }\end{array}$ \\
\hline Test $\mathrm{E}$ & Interaction & Posttest*HE & $\mathrm{CO}_{2} \mathrm{O} 1$ & .479 & & 98 & .027 \\
\hline Test E & Interaction & Posttest* HE & $\mathrm{CO}_{2} \mathrm{O} 4$ & .028 & & 68 & .002 \\
\hline
\end{tabular}

$\underline{\text { Test } \mathrm{F} \text { - High } \mathrm{ETCO}_{2}}$

Descriptive Statistics - Test F

\begin{tabular}{lccc}
\hline & Mean & Standard Deviation & $\mathrm{N}$ \\
\hline $\mathrm{O} 1$ & .14 & 6.755 & 21 \\
$\mathrm{O} 9$ & -.57 & 3.600 & 21 \\
$\mathrm{O} 4$ & .00 & 3.975 & 21 \\
$\mathrm{O} 7$ & 2.00 & 6.008 & 21 \\
\hline
\end{tabular}


Main \& Interaction Effects - Test F

\begin{tabular}{lcccccc}
\hline \multicolumn{1}{c}{ Source } & $\begin{array}{c}\text { Type III Sum of } \\
\text { Squares }\end{array}$ & df & $\begin{array}{c}\text { Mean } \\
\text { Square }\end{array}$ & F & Significance & $\begin{array}{c}\text { Partial } \\
\text { Eta } \\
\text { Squared }\end{array}$ \\
\hline Pretest & 30.964 & 1 & 30.964 & 1.543 & .229 & .072 \\
Method of & & & & & & \\
Recordkeeping & 8.679 & 1 & 8.679 & .363 & .554 & .018 \\
Pretest* Method & & & & & & \\
of \\
Recordkeeping
\end{tabular}


$\underline{\text { Test G - High } \mathrm{ETCO}_{2}}$

Descriptive Statistics - Test G

\begin{tabular}{lccc}
\hline & Mean & Standard Deviation & $\mathrm{N}$ \\
\hline $\mathrm{O} 1$ & .15 & 6.930 & 20 \\
$\mathrm{O} 2$ & .90 & 5.261 & 20 \\
$\mathrm{O} 4$ & -.35 & 3.731 & 20 \\
$\mathrm{O} 5$ & -.65 & 3.924 & 20 \\
\hline
\end{tabular}

Main \& Interaction Effects - Test G

\begin{tabular}{|c|c|c|c|c|c|c|}
\hline Source & $\begin{array}{c}\text { Type III Sum of } \\
\text { Squares }\end{array}$ & $\mathrm{df}$ & $\begin{array}{l}\text { Mean } \\
\text { Square }\end{array}$ & $\mathrm{F}$ & Significance & $\begin{array}{c}\text { Partial Eta } \\
\text { Squared }\end{array}$ \\
\hline Pretest & 21.013 & 1 & 21.013 & .755 & .396 & .038 \\
\hline Treatment & 1.013 & 1 & 1.013 & .054 & .819 & .003 \\
\hline Pretest* Treatment & 5.513 & 1 & 5.513 & .255 & .619 & .013 \\
\hline
\end{tabular}

\begin{tabular}{cccccc}
\hline & & & & & \\
\hline Test & Effect & Output & F & Significance & Partial Eta Squared \\
\hline Test G & Main & Pretest & .755 & .396 & .038 \\
Test G & Main & Treatment & .054 & .819 & .003 \\
Test G & Interaction & Pretest* Treatment & .255 & .619 & .013 \\
\hline
\end{tabular}




\section{Dependent Variable - Low $\mathrm{ETCO}_{2}$}

$\underline{\text { Tests A-D - Low } \text { ETCO }_{2}}$

Descriptive Statistics - Tests A-D

\begin{tabular}{lccc}
\hline & Mean & Standard Deviation & $\mathrm{N}$ \\
\hline O2 & -1.00 & 3.494 & 20 \\
O5 & .20 & 3.915 & 20 \\
O7 & -.30 & 1.949 & 20 \\
O9 & -.75 & 4.541 & 20 \\
\hline
\end{tabular}

Main \& Interaction Effects - Tests A-D

\begin{tabular}{|c|c|c|c|c|c|c|c|c|}
\hline \multicolumn{2}{|c|}{ Source } & $\begin{array}{l}\text { Type III Sum of } \\
\text { Squares }\end{array}$ & $\mathrm{df}$ & \multicolumn{2}{|c|}{$\begin{array}{l}\text { Mean } \\
\text { Square }\end{array}$} & $\mathrm{F}$ & Significance & $\begin{array}{c}\text { Partial } \\
\text { Eta } \\
\text { Squared }\end{array}$ \\
\hline \multicolumn{2}{|l|}{ Pretest } & .313 & 1 & \multicolumn{2}{|l|}{.313} & .018 & .895 & .001 \\
\hline \multicolumn{2}{|l|}{ Treatment } & 2.813 & 1 & \multicolumn{2}{|l|}{2.813} & .283 & .601 & .015 \\
\hline \multicolumn{2}{|c|}{ Pretest*Treatment } & 13.613 & 1 & 13.613 & & .774 & .390 & .039 \\
\hline Test & Effect & Out & & & $\mathrm{F}$ & & Significance & $\begin{array}{c}\text { Partial Eta } \\
\text { Squared }\end{array}$ \\
\hline Test A & Interaction & Pretest*T & reatmer & & .774 & & .390 & .039 \\
\hline Test B & Main & Treat & ment & & .283 & & .601 & .015 \\
\hline Test $\mathrm{C}$ & Main & Pre & & & .018 & & .895 & .001 \\
\hline Test D & Main & Treat & ment & & .283 & & .601 & .015 \\
\hline
\end{tabular}


$\underline{\text { Test E - Low ETCO }} \underline{2}_{2}$

Descriptive Statistics - Test E

\begin{tabular}{cccc}
\hline & Mean & Standard Deviation & $\mathrm{N}$ \\
\hline O2 & -1.00 & 3.494 & 20 \\
O5 & .20 & 3.915 & 20 \\
\hline
\end{tabular}

Main \& Interaction Effects - Test E

\begin{tabular}{lcccccc}
\hline \multicolumn{1}{c}{ Source } & $\begin{array}{c}\text { Type III Sum of } \\
\text { Squares }\end{array}$ & df & $\begin{array}{c}\text { Mean } \\
\text { Square }\end{array}$ & F & Significance & $\begin{array}{c}\text { Partial Eta } \\
\text { Squared }\end{array}$ \\
\hline Postest & 10.00 & 1 & 10.00 & .601 & .449 & .034 \\
Posttest*LETCO & \\
& 15.470 & 1 & 15.470 & .930 & .348 & .052 \\
Posttest*L LETCO $2 \mathrm{O} 4$ & 9.957 & 1 & 9.957 & .598 & .450 & .034 \\
\hline
\end{tabular}

\begin{tabular}{cccccc}
\hline Test & Effect & Output & F & Significance & $\begin{array}{c}\text { Partial Eta } \\
\text { Squared }\end{array}$ \\
\hline Test E & Interaction & Posttest*LETCO 201 & .930 & .348 & .052 \\
\multirow{2}{*}{ Test E } & Interaction & Posttest* L LETCO 204 & .598 & .450 & .034 \\
\hline
\end{tabular}

$\underline{\text { Test F - Low ETCO }} \underline{2}_{2}$

Descriptive Statistics - Test F

\begin{tabular}{cccc}
\hline O1 & Mean & Standard Deviation & $\mathrm{N}$ \\
\hline O9 & -.67 & 6.296 & 21 \\
O4 & -.71 & 4.429 & 21 \\
O7 & 1.00 & 3.130 & 21 \\
& -.29 & 1.901 & 21 \\
\hline
\end{tabular}


Main \& Interaction Effects - Test F

\begin{tabular}{|c|c|c|c|c|c|c|}
\hline Source & $\begin{array}{c}\text { Type III Sum of } \\
\text { Squares }\end{array}$ & df & $\begin{array}{l}\text { Mean } \\
\text { Square }\end{array}$ & $\mathrm{F}$ & Significance & $\begin{array}{l}\text { Partial } \\
\text { Eta } \\
\text { Squared }\end{array}$ \\
\hline Pretest & 23.048 & 1 & 23.048 & 1.251 & .277 & .059 \\
\hline $\begin{array}{l}\text { Method of } \\
\text { Recordkeeping }\end{array}$ & 9.333 & 1 & 9.333 & .405 & .532 & .020 \\
\hline Pretest* Method & & & & & & \\
\hline of & 8.048 & 1 & 8.048 & .507 & .485 & .025 \\
\hline Recordkeeping & & & & & & \\
\hline
\end{tabular}

\begin{tabular}{cccccc}
\hline Test & Effect & Output & F & Significance & $\begin{array}{c}\text { Partial Eta } \\
\text { Squared }\end{array}$ \\
\hline Test F & Main & Pretest & 1.251 & .277 & .059 \\
Test F & Main & Method of & & & \\
& & Recordkeeping & .405 & .532 & .020 \\
& & Pretest* & & \\
Test F & Interaction & Method of & .507 & .485 & .025 \\
& & Recordkeeping & & \\
& & &
\end{tabular}


$\underline{\text { Test G - Low ETCO }} 2$

Descriptive Statistics - Test G

\begin{tabular}{lccc}
\hline & Mean & Standard Deviation & $\mathrm{N}$ \\
\hline O1 & -.80 & 6.429 & 20 \\
$\mathrm{O} 2$ & -1.0 & 3.494 & 20 \\
$\mathrm{O} 4$ & .95 & 3.203 & 20 \\
$\mathrm{O} 5$ & .20 & 3.915 & 20 \\
\hline
\end{tabular}

Main \& Interaction Effects - Test G

\begin{tabular}{|c|c|c|c|c|c|c|c|}
\hline \multicolumn{2}{|c|}{ Source } & $\begin{array}{l}\text { Type III Sum of } \\
\text { Squares }\end{array}$ & df & $\begin{array}{l}\text { Mean } \\
\text { Square }\end{array}$ & $\mathrm{F}$ & Significance & $\begin{array}{c}\text { Partial Eta } \\
\text { Squared }\end{array}$ \\
\hline \multicolumn{2}{|l|}{ Pretest } & 43.513 & 1 & 43.513 & 2.416 & .137 & 113 \\
\hline \multicolumn{2}{|l|}{ Treatment } & 4.513 & 1 & 4.513 & .188 & .670 & .010 \\
\hline \multicolumn{2}{|c|}{ Pretest* Treatment } & 1.513 & 1 & 1.513 & .066 & .801 & .066 \\
\hline Test & Effect & \multicolumn{2}{|c|}{ Output } & $\mathrm{F}$ & \multicolumn{2}{|c|}{ Significance } & 1 Eta Squared \\
\hline Test G & Main & \multicolumn{2}{|c|}{ Pretest } & 2.416 & \multicolumn{2}{|c|}{.137} & .113 \\
\hline Test G & Main & \multicolumn{2}{|c|}{ Treatment } & .188 & \multicolumn{2}{|c|}{.670} & .010 \\
\hline Test G & Interaction & \multicolumn{2}{|c|}{ Pretest* Treatment } & .066 & \multicolumn{2}{|c|}{.801} & .066 \\
\hline
\end{tabular}




\section{Dependent Variable - Low $\mathrm{SPO}_{2}$}

Tests A-D - Low $\mathrm{SPO}_{2}$

Descriptive Statistics - Tests A-D

\begin{tabular}{lccc}
\hline O2 & Mean & Standard Deviation & $\mathrm{N}$ \\
\hline O5 & -3.05 & 8.363 & 20 \\
O7 & -2.20 & 6.717 & 20 \\
O9 & -1.25 & 3.522 & 20 \\
& -.55 & 1.820 & 20 \\
\hline
\end{tabular}

Main \& Interaction Effects - Tests A-D

\begin{tabular}{|c|c|c|c|c|c|c|c|}
\hline \multicolumn{2}{|c|}{ Source } & $\begin{array}{l}\text { Type III Sum of } \\
\text { Squares }\end{array}$ & $\mathrm{df}$ & $\begin{array}{l}\text { Mean } \\
\text { Square }\end{array}$ & $\mathrm{F}$ & Significance & $\begin{array}{l}\text { Partial } \\
\text { Eta } \\
\text { Squared }\end{array}$ \\
\hline \multicolumn{2}{|l|}{ Pretest } & 59.513 & 1 & 59.513 & 3.228 & .088 & .145 \\
\hline \multicolumn{2}{|l|}{ Treatment } & 12.013 & 1 & 12.013 & .232 & .635 & .012 \\
\hline \multicolumn{2}{|c|}{ Pretest*Treatment } & .113 & 1 & .113 & .006 & .938 & .000 \\
\hline Test & Effect & \multicolumn{2}{|c|}{ Output } & \multicolumn{2}{|c|}{$\mathrm{F}$} & Significance & $\begin{array}{c}\text { Partial Eta } \\
\text { Squared }\end{array}$ \\
\hline Test A & Interaction & \multicolumn{2}{|c|}{ Pretest*Treatment } & \multicolumn{2}{|c|}{.006} & .938 & .000 \\
\hline Test B & Main & \multicolumn{2}{|c|}{ Treatment } & \multicolumn{2}{|c|}{.232} & .635 & .012 \\
\hline Test C & Main & \multicolumn{2}{|c|}{ Pretest } & \multicolumn{2}{|c|}{3.228} & .088 & .145 \\
\hline Test D & Main & \multicolumn{2}{|c|}{ Treatment } & \multicolumn{2}{|c|}{.232} & .635 & .012 \\
\hline
\end{tabular}


$\underline{\text { Test E - Low } \mathrm{SPO}_{2}}$

Descriptive Statistics - Test E

\begin{tabular}{cccc}
\hline & Mean & Standard Deviation & N \\
\hline O2 & -3.05 & 8.363 & 20 \\
O5 & -2.20 & 6.717 & 20 \\
\hline
\end{tabular}

Main \& Interaction Effects - Test E

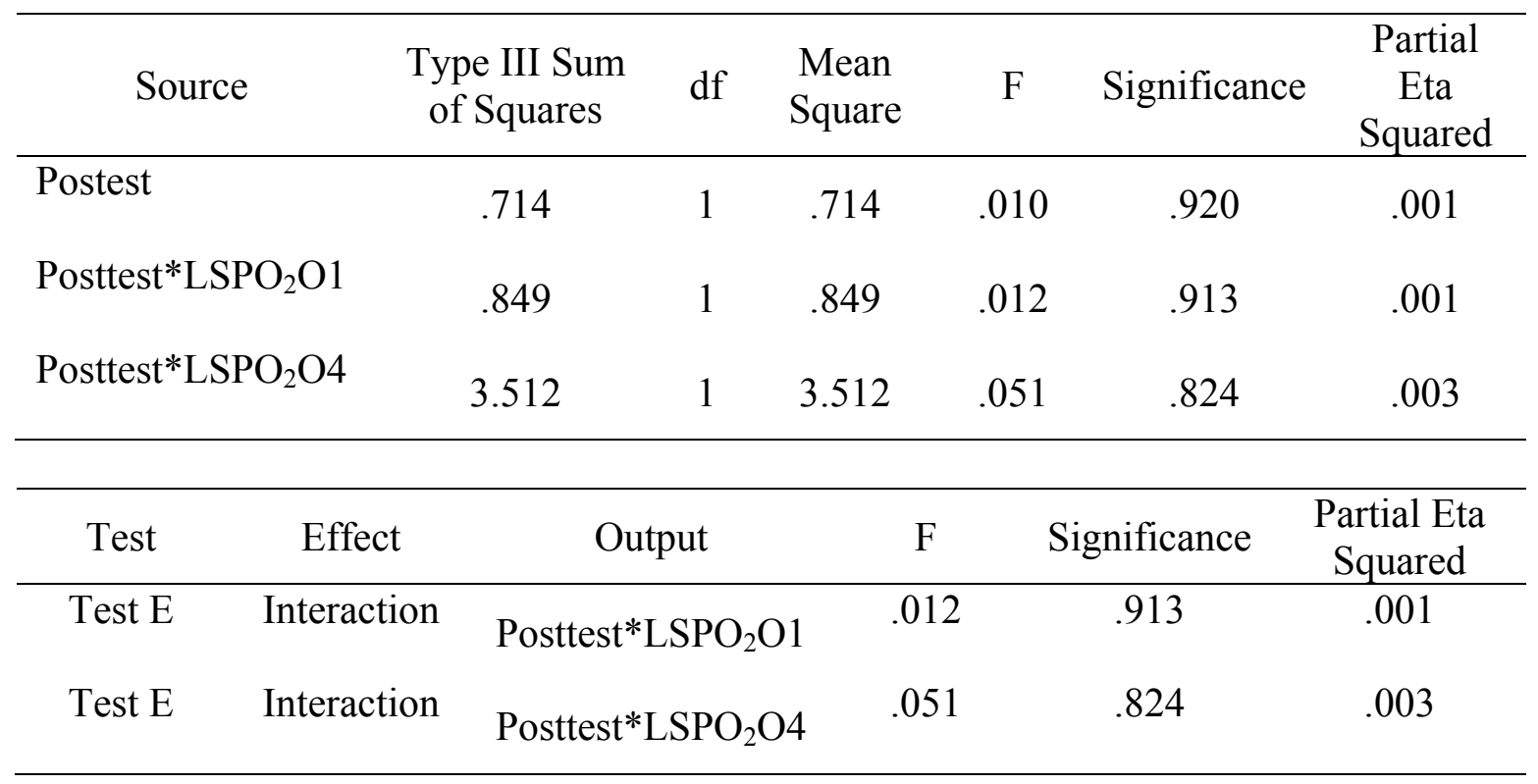

$\underline{\text { Test F - Low } \mathrm{SPO}_{2}}$

Descriptive Statistics - Test F

\begin{tabular}{lccc}
\hline & Mean & Standard Deviation & $\mathrm{N}$ \\
\hline O1 & -.43 & 1.363 & 21 \\
O9 & -.62 & 1.802 & 21 \\
O4 & -1.24 & 2.343 & 21 \\
O7 & -1.10 & 3.506 & 21 \\
\hline
\end{tabular}


Main \& Interaction Effects - Test F

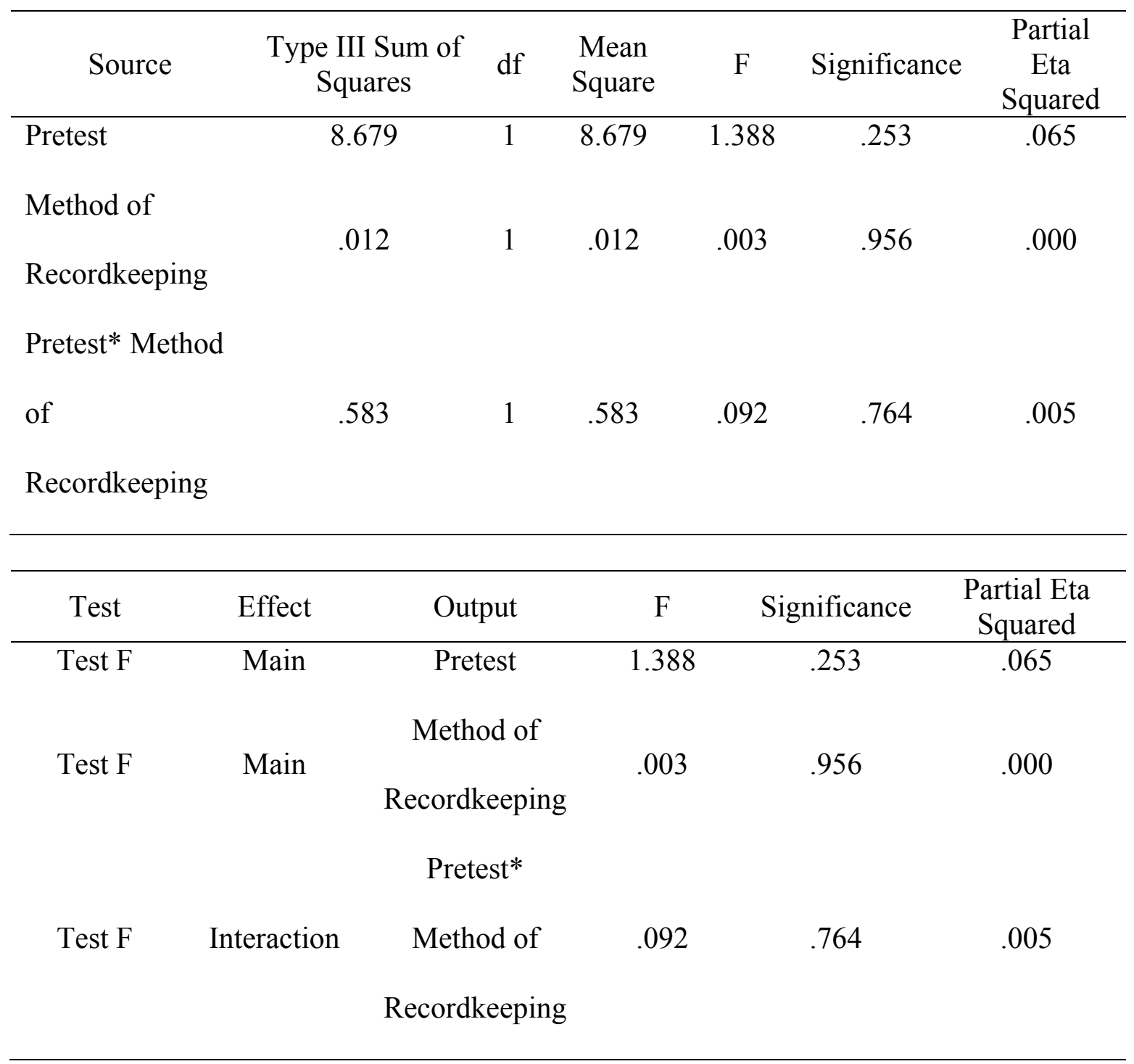


$\underline{\text { Test G - Low } \mathrm{SPO}_{2}}$

Descriptive Statistics - Test G

\begin{tabular}{lccc}
\hline & Mean & Standard Deviation & $\mathrm{N}$ \\
\hline O1 & -11.45 & 22.322 & 20 \\
O2 & -3.05 & 8.363 & 20 \\
O4 & -1.35 & 2.346 & 20 \\
O5 & -2.20 & 6.717 & 20 \\
\hline
\end{tabular}

Main \& Interaction Effects - Test G

\begin{tabular}{|c|c|c|c|c|c|c|c|}
\hline \multicolumn{2}{|c|}{ Source } & $\begin{array}{c}\text { Type III Sum of } \\
\text { Squares }\end{array}$ & $\mathrm{df}$ & $\begin{array}{c}\text { Mean } \\
\text { Square }\end{array}$ & $\mathrm{F}$ & Significance & $\begin{array}{l}\text { Partial Eta } \\
\text { Squared }\end{array}$ \\
\hline \multicolumn{2}{|l|}{ Pretest } & 599.513 & 1 & 599.513 & 3.885 & .063 & .170 \\
\hline \multicolumn{2}{|l|}{ Treatment } & 285.013 & 1 & 285.013 & 1.548 & .229 & .075 \\
\hline \multicolumn{2}{|c|}{ Pretest* Treatment } & 427.813 & 1 & 427.813 & 2.948 & .102 & .134 \\
\hline Test & Effect & \multicolumn{2}{|c|}{ Output } & $\mathrm{F}$ & \multicolumn{2}{|c|}{ Significance } & 1 Eta Squared \\
\hline Test G & Main & \multicolumn{2}{|c|}{ Pretest } & 3.885 & \multicolumn{2}{|c|}{.063} & .170 \\
\hline Test G & Main & \multicolumn{2}{|c|}{ Treatment } & 1.548 & \multicolumn{2}{|c|}{.229} & .075 \\
\hline Test G & Interaction & \multicolumn{2}{|c|}{ Pretest* Treatment } & 2.948 & \multicolumn{2}{|c|}{.102} & .134 \\
\hline
\end{tabular}




\section{Dependent Variable - High Inspiratory Pressure}

Tests A-D - High Inspiratory Pressure

Descriptive Statistics - Tests A-D

\begin{tabular}{lccc}
\hline & Mean & Standard Deviation & $\mathrm{N}$ \\
\hline O2 & 2.60 & 5.051 & 20 \\
O5 & 1.00 & 4.668 & 20 \\
O7 & .15 & 6.620 & 20 \\
O9 & 2.00 & 3.112 & 20 \\
\hline
\end{tabular}

Main \& Interaction Effects - Tests A-D

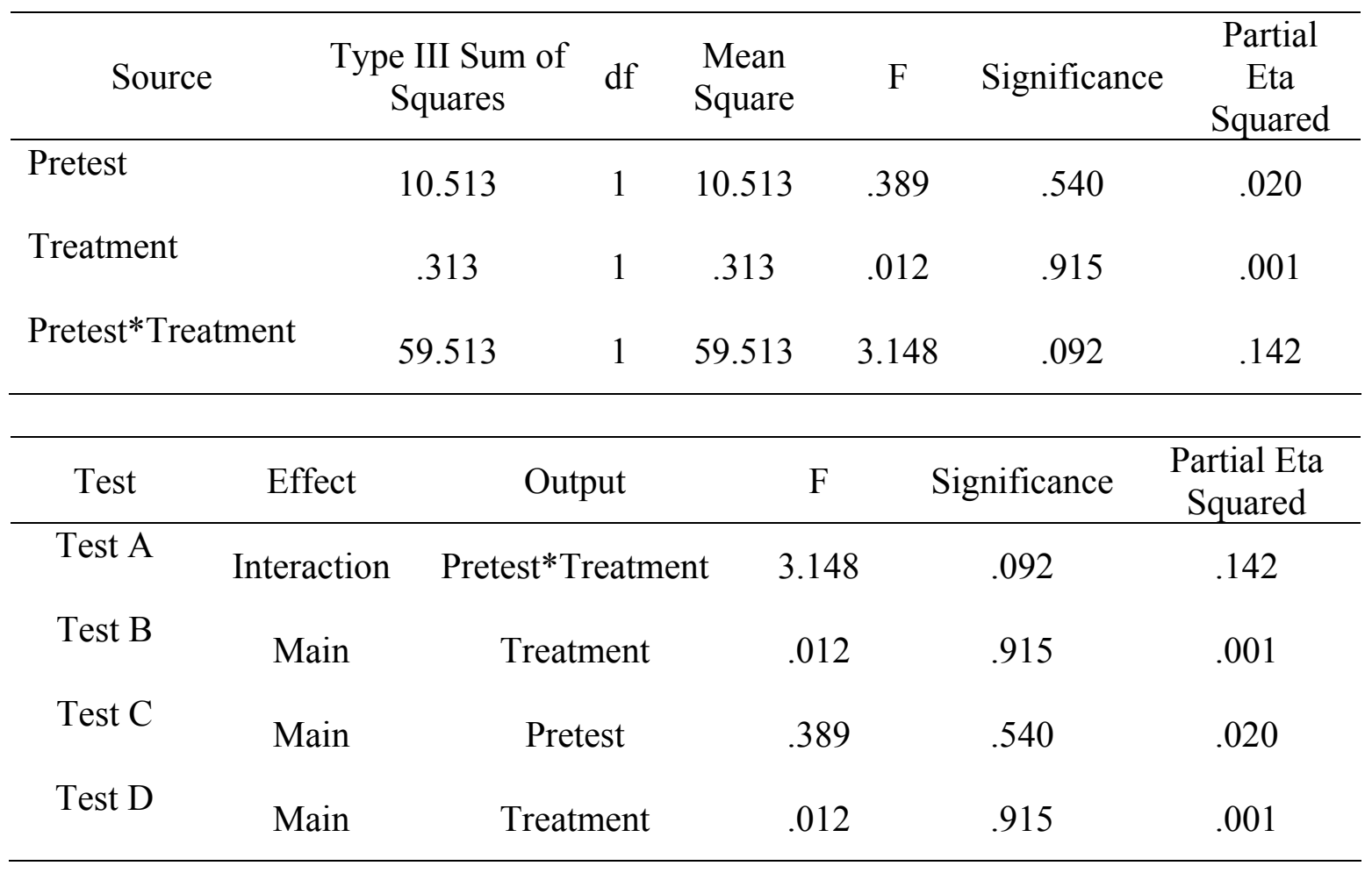


$\underline{\text { Test E - High Inspiratory Pressure }}$

Descriptive Statistics - Test E

\begin{tabular}{cccc}
\hline & Mean & Standard Deviation & $\mathrm{N}$ \\
\hline $\mathrm{O} 2$ & 2.60 & 5.051 & 20 \\
$\mathrm{O} 5$ & 1.00 & 4.668 & 20 \\
\hline
\end{tabular}

Main \& Interaction Effects - Test E

\begin{tabular}{|c|c|c|c|c|c|c|c|}
\hline Source & \multicolumn{2}{|c|}{$\begin{array}{c}\text { Type III Sum of } \\
\text { Squares }\end{array}$} & $\mathrm{df}$ & $\begin{array}{c}\text { Mean } \\
\text { Square }\end{array}$ & $\mathrm{F}$ & Significance & $\begin{array}{c}\text { Partial } \\
\text { Eta } \\
\text { Squared }\end{array}$ \\
\hline Postest & \multicolumn{2}{|c|}{17.278} & 1 & 17.278 & .671 & .424 & .038 \\
\hline Posttest*HIPO1 & \multicolumn{2}{|c|}{.905} & 1 & .905 & .035 & .853 & .002 \\
\hline Posttest*HIPO4 & \multicolumn{2}{|c|}{14.239} & 1 & 14.239 & .553 & .467 & .032 \\
\hline Test & Effect & $\mathrm{Ou}$ & & & & ignificance & $\begin{array}{c}\text { Partial Eta } \\
\text { Squared }\end{array}$ \\
\hline Test E & Interaction & Posttest & $\mathrm{HIPO}$ & & & .853 & .002 \\
\hline Test E & Interaction & Posttest & $\mathrm{HIPO}_{2}$ & & & .467 & .032 \\
\hline
\end{tabular}

$\underline{\text { Test F - High Inspiratory Pressure }}$

Descriptive Statistics - Test F

\begin{tabular}{lccc}
\hline & Mean & Standard Deviation & $\mathrm{N}$ \\
\hline O1 & -.29 & 5.951 & 21 \\
O9 & 2.05 & 3.041 & 21 \\
O4 & .95 & 4.031 & 21 \\
O7 & .57 & 6.735 & 21 \\
\hline
\end{tabular}


Main \& Interaction Effects - Test F

\begin{tabular}{|c|c|c|c|c|c|c|}
\hline Source & $\begin{array}{l}\text { Type III Sum of } \\
\text { Squares }\end{array}$ & df & $\begin{array}{l}\text { Mean } \\
\text { Square }\end{array}$ & $\mathrm{F}$ & Significance & $\begin{array}{c}\text { Partial } \\
\text { Eta } \\
\text { Squared }\end{array}$ \\
\hline Pretest & 298 & 1 & .298 & .009 & .924 & .000 \\
\hline \multicolumn{7}{|l|}{ Method of } \\
\hline Recordkeeping & 20.012 & 1 & 20.012 & .649 & .430 & .031 \\
\hline \multicolumn{7}{|l|}{ Pretest* Method } \\
\hline of & 38.679 & 1 & 38.679 & 2.255 & .149 & .101 \\
\hline Recordkeeping & & & & & & \\
\hline
\end{tabular}

\begin{tabular}{cccccc}
\hline Test & Effect & Output & F & Significance & $\begin{array}{c}\text { Partial Eta } \\
\text { Squared }\end{array}$ \\
\hline Test F & Main & Pretest & .009 & .924 & .000 \\
Test F & Main & Method of & & & \\
& & Recordkeeping & .649 & .430 & .031 \\
& & Pretest* & & \\
Test F & Interaction & Method of & 2.255 & .149 & .101 \\
& & Recordkeeping & & \\
\end{tabular}


$\underline{\text { Test G - High Inspiratory Pressure }}$

Descriptive Statistics - Test G

\begin{tabular}{lccc}
\hline & Mean & Standard Deviation & $\mathrm{N}$ \\
\hline O1 & -.30 & 6.105 & 20 \\
O2 & 2.60 & 5.051 & 20 \\
O4 & .90 & 4.128 & 20 \\
O5 & 1.00 & 4.668 & 20 \\
\hline
\end{tabular}

Main \& Interaction Effects - Test G

\begin{tabular}{|c|c|c|c|c|c|c|c|}
\hline Source & Type & $\begin{array}{l}\text { I Sum of } \\
\text { uares }\end{array}$ & $\mathrm{df}$ & $\begin{array}{l}\text { Mean } \\
\text { Square }\end{array}$ & $\mathrm{F}$ & Significance & $\begin{array}{l}\text { Partial Eta } \\
\text { Squared }\end{array}$ \\
\hline Pretest & & 300 & 1 & .800 & .033 & .858 & .002 \\
\hline Treatment & & .000 & 1 & 45.000 & 2.426 & .136 & .113 \\
\hline Pretest* Treatment & & .200 & 1 & 39.200 & 1.418 & 248 & .069 \\
\hline Test & Effect & \multicolumn{2}{|c|}{ Output } & $\mathrm{F}$ & & nificance & 1 Eta Squared \\
\hline Test $\mathrm{G}$ & Main & \multicolumn{2}{|c|}{ Pretest } & .033 & & .858 & .002 \\
\hline Test G & Main & \multicolumn{2}{|c|}{ Treatment } & 2.426 & & .136 & .113 \\
\hline Test G & nteraction & \multicolumn{2}{|c|}{ Pretest* Treatment } & 1.418 & & .248 & .069 \\
\hline
\end{tabular}




\section{Dependent Variable - Low Inspiratory Pressure}

Tests A-D - Low Inspiratory Pressure

Descriptive Statistics - Tests A-D

\begin{tabular}{lccc}
\hline O2 & Mean & Standard Deviation & N \\
\hline O5 & -1.95 & 4.968 & 20 \\
O7 & .30 & 4.041 & 20 \\
O9 & 2.25 & 7.820 & 20 \\
& 1.35 & 5.706 & 20 \\
\hline
\end{tabular}

Main \& Interaction Effects - Tests A-D

\begin{tabular}{|c|c|c|c|c|c|c|c|}
\hline \multicolumn{2}{|c|}{ Source } & $\begin{array}{l}\text { Type III Sum } \\
\text { of Squares }\end{array}$ & $\mathrm{df}$ & $\begin{array}{l}\text { Mean } \\
\text { Square }\end{array}$ & $\mathrm{F}$ & Significance & $\begin{array}{c}\text { Partial } \\
\text { Eta } \\
\text { Squared }\end{array}$ \\
\hline \multicolumn{2}{|l|}{ Pretest } & 137.813 & 1 & 137.813 & 3.047 & .097 & .138 \\
\hline \multicolumn{2}{|l|}{ Treatment } & 9.113 & 1 & 9.113 & .478 & .498 & .025 \\
\hline \multicolumn{2}{|c|}{ Pretest*Treatment } & 49.613 & 1 & 49.613 & 1.395 & .252 & .068 \\
\hline Test & Effect & \multicolumn{2}{|c|}{ Output } & \multicolumn{2}{|c|}{$\mathrm{F}$} & ignificance & $\begin{array}{c}\text { Partial Eta } \\
\text { Squared }\end{array}$ \\
\hline Test A & Interaction & \multicolumn{2}{|c|}{ Pretest*Treatmen } & \multicolumn{2}{|c|}{1.395} & .252 & .068 \\
\hline Test B & Main & \multicolumn{2}{|c|}{ Treatment } & \multicolumn{2}{|c|}{.478} & .498 & .025 \\
\hline Test C & Main & \multicolumn{2}{|c|}{ Pretest } & \multicolumn{2}{|c|}{3.047} & .097 & .138 \\
\hline Test D & Main & \multicolumn{2}{|c|}{ Treatment } & \multicolumn{2}{|c|}{1.395} & .252 & .068 \\
\hline
\end{tabular}


$\underline{\text { Test E - Low Heart Rate }}$

Descriptive Statistics - Test E

\begin{tabular}{cccc}
\hline & Mean & Standard Deviation & N \\
\hline O2 & -1.95 & 4.968 & 20 \\
O5 & .30 & 4.041 & 20 \\
\hline
\end{tabular}

Main \& Interaction Effects - Test E

\begin{tabular}{|c|c|c|c|c|c|c|c|}
\hline Source & \multicolumn{2}{|c|}{$\begin{array}{c}\text { Type III Sum of } \\
\text { Squares }\end{array}$} & $\mathrm{df}$ & $\begin{array}{l}\text { Mean } \\
\text { Square }\end{array}$ & $\mathrm{F}$ & Significance & $\begin{array}{c}\text { Partial } \\
\text { Eta } \\
\text { Squared }\end{array}$ \\
\hline Postest & \multicolumn{2}{|c|}{46.399} & 1 & 46.399 & 1.952 & .180 & .103 \\
\hline Posttest*LIPO1 & \multicolumn{2}{|c|}{22.761} & 1 & 22.761 & .958 & .342 & .053 \\
\hline Posttest*LIPO4 & \multicolumn{2}{|c|}{1.334} & 1 & 1.334 & .056 & .816 & .003 \\
\hline Test & Effect & $\mathrm{Ou}$ & put & & & Significance & $\begin{array}{c}\text { Partial Eta } \\
\text { Squared }\end{array}$ \\
\hline Test E & Interaction & Posttes & *LIPO1 & & & .342 & .053 \\
\hline Test E & Interaction & Posttes & $*$ LIPO4 & & & .816 & .003 \\
\hline
\end{tabular}

$\underline{\text { Test F - Low Inspiratory Pressure }}$

Descriptive Statistics - Test F

\begin{tabular}{cccc}
\hline & Mean & Standard Deviation & $\mathrm{N}$ \\
\hline O1 & 1.24 & 4.582 & 21 \\
$\mathrm{O} 4$ & 1.29 & 5.569 & 21 \\
$\mathrm{O} 7$ & -.90 & 2.143 & 21 \\
& 2.52 & 7.724 & 21 \\
\hline
\end{tabular}


Main \& Interaction Effects - Test F

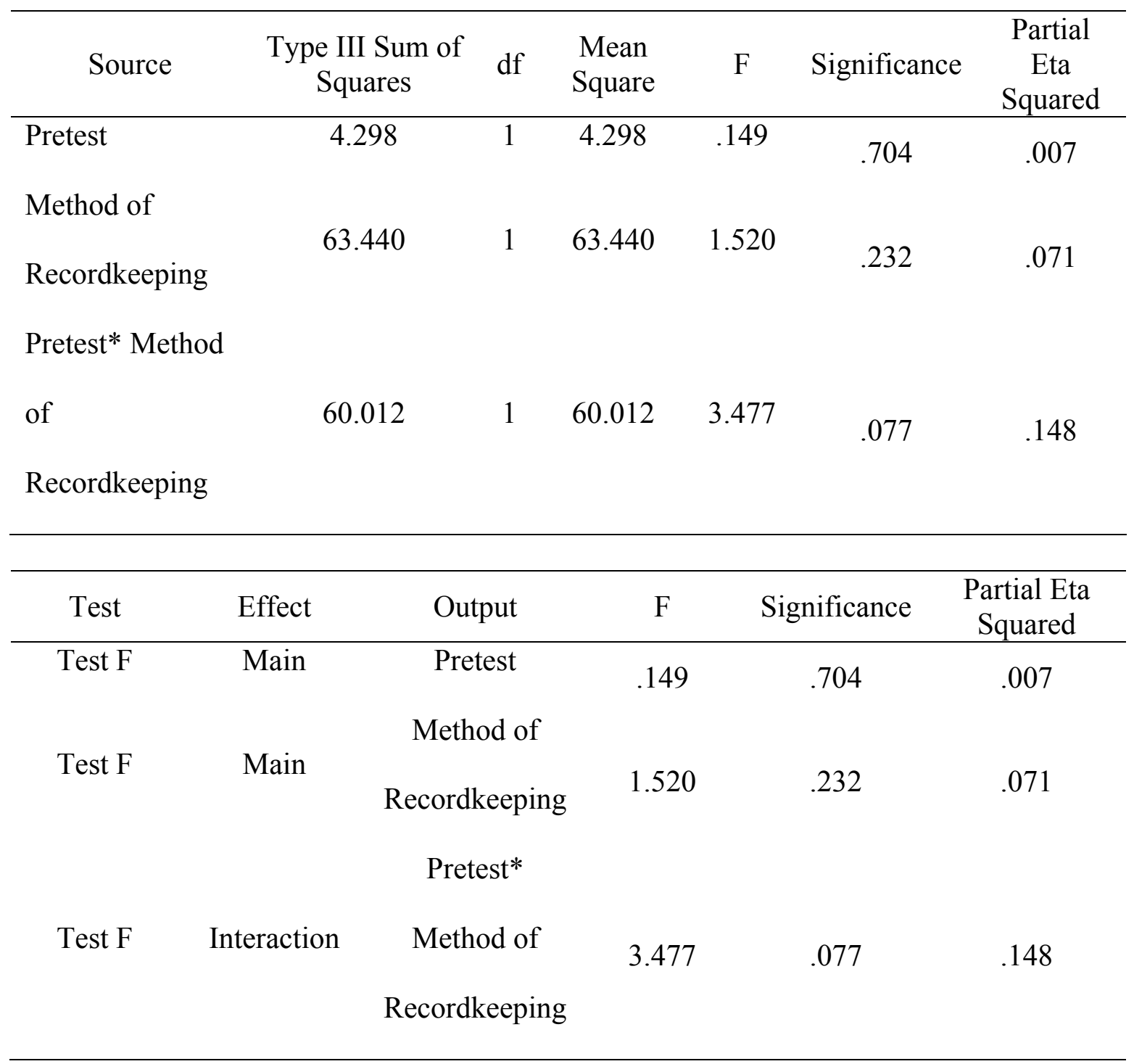


$\underline{\text { Test G - Low Inspiratory Pressure }}$

Descriptive Statistics - Test G

\begin{tabular}{lccc}
\hline & Mean & Standard Deviation & $\mathrm{N}$ \\
\hline O1 & 1.15 & 4.682 & 20 \\
O2 & -1.95 & 4.968 & 20 \\
O4 & -1.05 & 2.089 & 20 \\
O5 & .30 & 4.041 & 20 \\
\hline
\end{tabular}

Main \& Interaction Effects - Test G

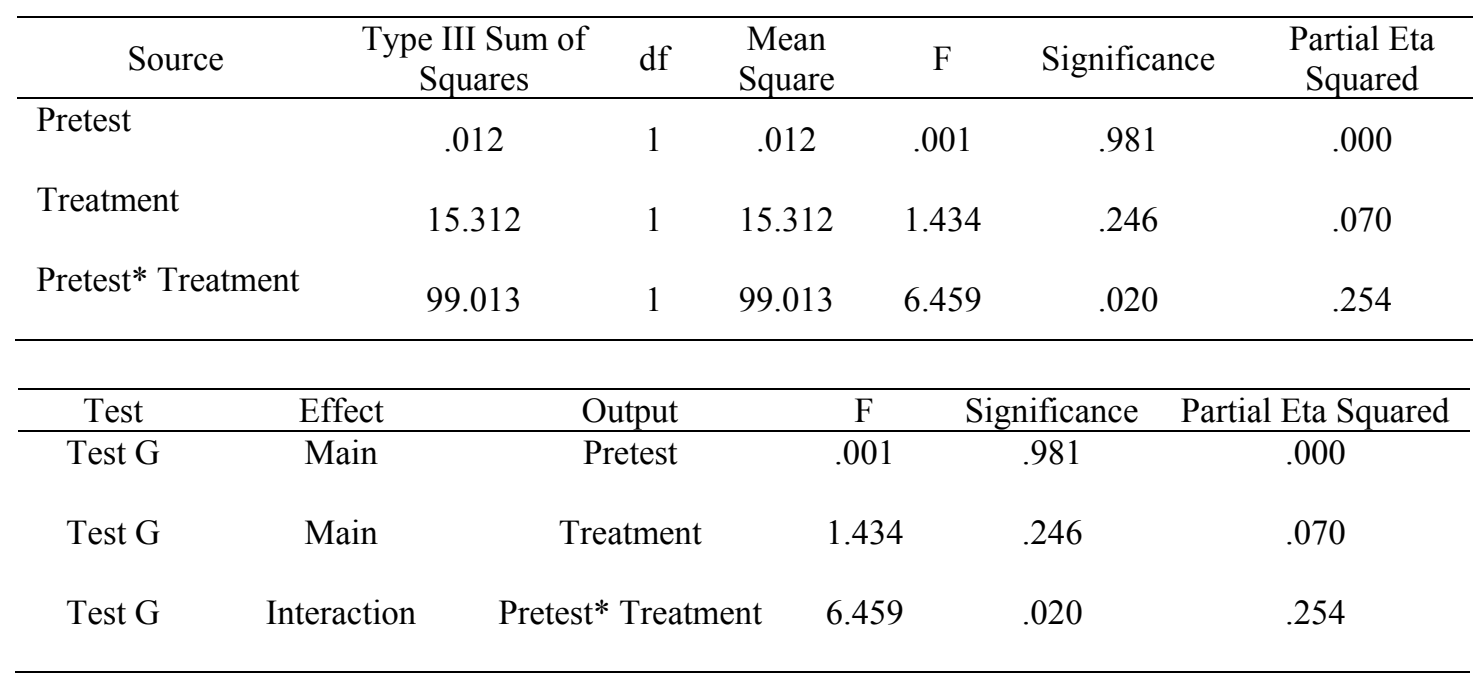




\section{Dependent Variable - Total Fluid Volume}

Tests A-D - Total Fluid Volume

Descriptive Statistics - Tests A-D

\begin{tabular}{lccc}
\hline O2 & Mean & Standard Deviation & $\mathrm{N}$ \\
\hline O5 & 22.50 & 254.680 & 20 \\
O7 & -65.50 & 223.994 & 20 \\
O9 & 62.50 & 379.014 & 20 \\
& -15.00 & 146.987 & 20 \\
\hline
\end{tabular}

Main \& Interaction Effects - Tests A-D

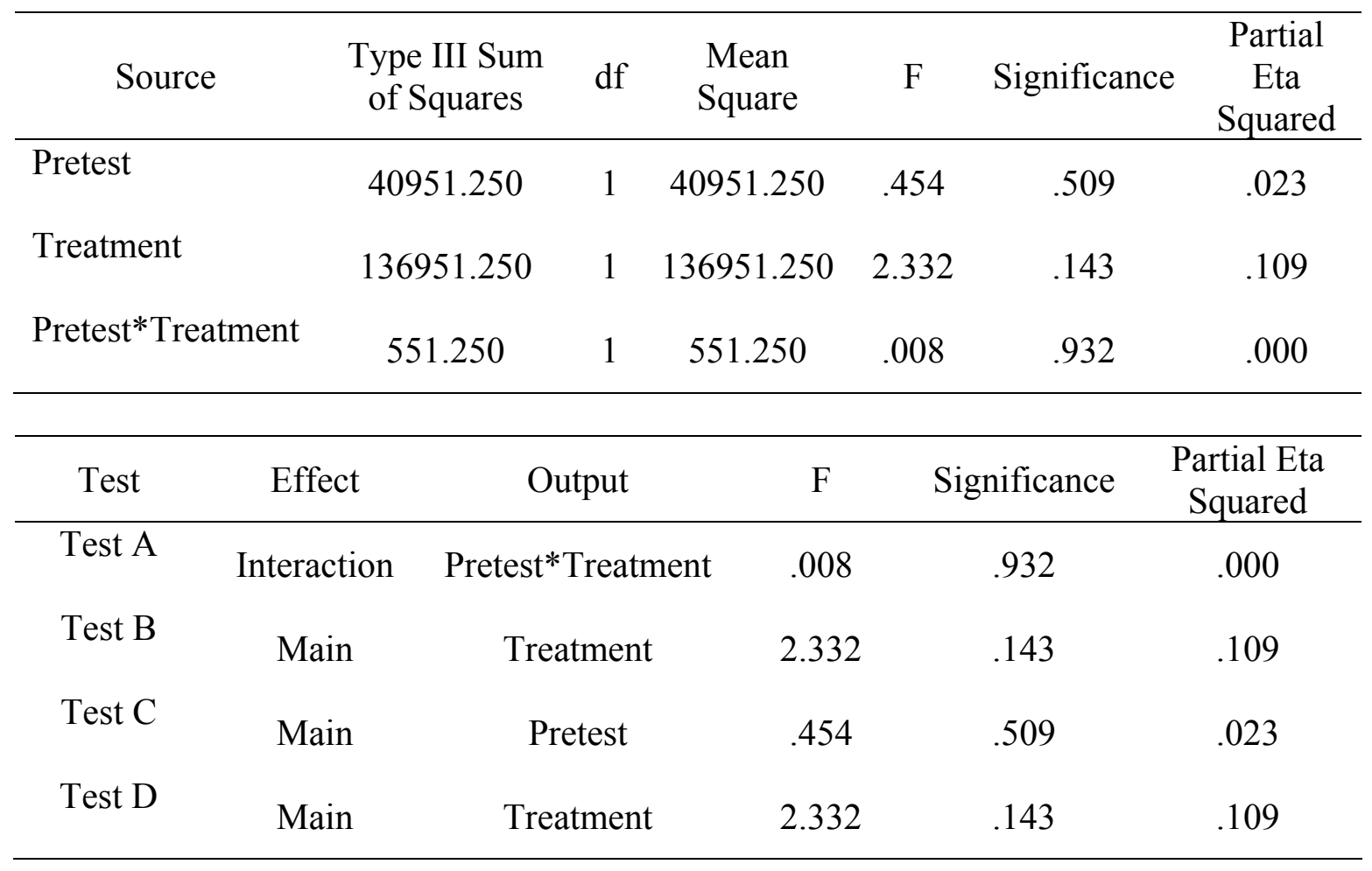


$\underline{\text { Test E - Total Fluid Volume }}$

Descriptive Statistics - Test E

\begin{tabular}{cccc}
\hline & Mean & Standard Deviation & N \\
\hline O2 & 22.50 & 254.680 & 20 \\
O5 & -65.50 & 223.994 & 20 \\
\hline
\end{tabular}

Main \& Interaction Effects - Test E

\begin{tabular}{|c|c|c|c|c|c|c|c|c|c|}
\hline \multicolumn{2}{|l|}{ Source } & \multicolumn{2}{|c|}{$\begin{array}{c}\text { Type III Sum } \\
\text { of Squares }\end{array}$} & $\mathrm{df}$ & \multicolumn{2}{|c|}{$\begin{array}{l}\text { Mean } \\
\text { Square }\end{array}$} & $\mathrm{F}$ & Significance & $\begin{array}{c}\text { Partial } \\
\text { Eta } \\
\text { Squared }\end{array}$ \\
\hline \multicolumn{2}{|l|}{ Postest } & \multicolumn{2}{|c|}{40465.538} & 1 & \multicolumn{2}{|c|}{40465.538} & .865 & .365 & .048 \\
\hline \multicolumn{2}{|c|}{ Posttest*TFVO1 } & \multicolumn{2}{|c|}{353544.614} & 1 & \multicolumn{2}{|c|}{353544.614} & 7.560 & .014 & .308 \\
\hline \multicolumn{2}{|c|}{ Posttest*TFVO4 } & \multicolumn{2}{|c|}{52729.232} & 1 & \multicolumn{2}{|c|}{52729.232} & 1.127 & .303 & .062 \\
\hline Test & \multicolumn{2}{|c|}{ Effect } & \multicolumn{3}{|c|}{ Output } & $\mathrm{F}$ & \multicolumn{2}{|c|}{ Significance } & $\begin{array}{l}\text { Partial Eta } \\
\text { Squared }\end{array}$ \\
\hline Test E & \multicolumn{2}{|c|}{ Interaction } & \multicolumn{3}{|c|}{ Posttest*TFVO1 } & 7.560 & \multicolumn{2}{|r|}{.014} & .308 \\
\hline Test E & \multicolumn{2}{|c|}{ Interaction } & \multicolumn{3}{|c|}{ Posttest*TFVO4 } & 1.127 & \multicolumn{2}{|r|}{.303} & .062 \\
\hline
\end{tabular}

$\underline{\text { Test F - Total Fluid Volume }}$

Descriptive Statistics - Test F

\begin{tabular}{cccc}
\hline & Mean & Standard Deviation & N \\
\hline O1 & 63.81 & 238.337 & 21 \\
O4 & -11.90 & 143.966 & 21 \\
O7 & -88.10 & 241.819 & 21 \\
& 59.52 & 369.668 & 21 \\
\hline
\end{tabular}


Main \& Interaction Effects - Test F

\begin{tabular}{|c|c|c|c|c|c|c|}
\hline Source & $\begin{array}{c}\text { Type III Sum of } \\
\text { Squares }\end{array}$ & $\mathrm{df}$ & $\begin{array}{l}\text { Mean } \\
\text { Square }\end{array}$ & $\mathrm{F}$ & Significance & $\begin{array}{c}\text { Partial } \\
\text { Eta } \\
\text { Squared }\end{array}$ \\
\hline Pretest & 34001.190 & 1 & 34001.190 & .604 & .446 & .029 \\
\hline $\begin{array}{l}\text { Method of } \\
\text { Recordkeeping }\end{array}$ & 27144.048 & 1 & 27144.048 & .434 & .517 & .021 \\
\hline Pretest* Method & & & & & & \\
\hline of & 261858.333 & 1 & 261858.333 & 3.305 & .084 & .142 \\
\hline Recordkeeping & & & & & & \\
\hline
\end{tabular}

\begin{tabular}{cccccc}
\hline Test & Effect & Output & F & Significance & $\begin{array}{c}\text { Partial Eta } \\
\text { Squared }\end{array}$ \\
\hline Test F & Main & Pretest & .604 & .446 & .029 \\
Test F & Main & Method of & & .517 & .021 \\
& & Recordkeeping & .434 & & \\
Test F & Interaction & Pretest* & & .142 \\
& & Method of & 3.305 & .084 & \\
& & Recordkeeping & & \\
\hline
\end{tabular}


$\underline{\text { Test G - Total Fluid Volume }}$

Descriptive Statistics - Test G

\begin{tabular}{cccc}
\hline O1 & Mean & Standard Deviation & $\mathrm{N}$ \\
\hline O2 & 57.00 & 242.424 & 20 \\
O4 & 22.50 & 254.680 & 20 \\
O5 & -87.50 & 248.085 & 20 \\
& -65.50 & 223.994 & 20 \\
\hline
\end{tabular}

Main \& Interaction Effects - Test G

\begin{tabular}{|c|c|c|c|c|c|c|c|}
\hline \multicolumn{2}{|c|}{ Source } & $\begin{array}{c}\text { Type III Sum of } \\
\text { Squares }\end{array}$ & $\mathrm{df}$ & $\begin{array}{l}\text { Mean } \\
\text { Square }\end{array}$ & $\mathrm{F}$ & Significance & $\begin{array}{l}\text { Partial Eta } \\
\text { Squared }\end{array}$ \\
\hline \multicolumn{2}{|l|}{ Pretest } & 270281.250 & 1 & 270281.250 & 3.244 & .088 & .146 \\
\hline \multicolumn{2}{|l|}{ Treatment } & 781.250 & 1 & 781.250 & .017 & .899 & .001 \\
\hline \multicolumn{2}{|c|}{ Pretest* Treatment } & 15961.250 & 1 & 15961.250 & .325 & .575 & .017 \\
\hline Test & Effect & Outpu & & $\mathrm{F}$ & Sigr & icance & Eta Squared \\
\hline Test $\mathrm{G}$ & Main & Pretes & & 3.244 & & 88 & .146 \\
\hline Test G & Main & Treatme & & .017 & & 99 & .001 \\
\hline Test G & Interaction & Pretest* Tre & ment & .325 & & 75 & .017 \\
\hline
\end{tabular}


APPENDIX G: IRB APPROVAL 


\section{VCUMemo

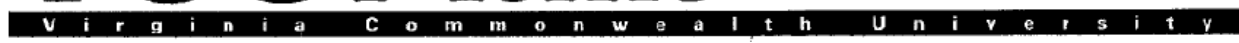

Office of Research Subjects Protection

BioTechnology Research Park

BioTech One, 800 E. Leigh Street, \#114

P.O. Box 980568

Richmond, Virginia 23298-0568

DATE: $\quad$ December 21, 2009

(804) 828-0868, fax (804) 827-1448

TO: $\quad$ Clarence J. Biddle, $\mathrm{PhD}$

Nurse Anesthesia, $\mathrm{PhD}$

Box 980226

FROM: Lloyd H. Byrd, MS $\quad$ B $/ \not x$

Chairperson, VCU IRB Panel $E$

Box 980568

RE:

VCU IRB \#: HM12567

Title: Anesthesia Recordkeeping Accuracy of Recall with Computerized and Manual Entry

Recordkeeping

On December 18, 2009 the following research study qualified for exemption according to 45 CFR 46.101(b) Category 2.

This approval includes the following items reviewed by this Panel:

RESEARCII APPLICATION/PROPOSAL: NONE

PROTOCOL: Anesthesia Recordkeeping Accuracy of Recall with Computerized and Manual Entry Recordkeeping ,version $10 / 26 / 09$, received $11 / 2 / 09$

\section{CONSENT/ASSENT:}

- Because the project is exempt from federal regulations, the procedures described in $\S 46.116$ (Consent) and 46.117 (Documentation of Consent) are not applicable to your research study. Nevertheless, the Common Law of the Commonwealth of Virginia, as well as the canons of sound ethics require you to inform potential subjects of foreseeable risks and possible benefits (if any) associated with participation in your research study. Therefore potential subjects should be informed of foreseeable risks and possible benefits of participation in your research study. They should also be informed that they may refuse to participate in your research and they should understand that they might withdraw at any time without penalty.

- This process of informed decision-making should be documented along with other information associated with the study.

\section{ADDITIONAL DOCUMENTS:}

- None

The Primary Reviewer assigned to your rescarch study is Gwendolyn Parker, MS, FNP-C. If you have any questions, plcasc contact Ms. Parker at ggparker@ycu.edu and 828-5090; or you may contact Donna Gross, IRB Coordinator, VCU Office of Research Subjects Protection, at dsgross@vcu.edu or 827-2261. 
June 9, 2010

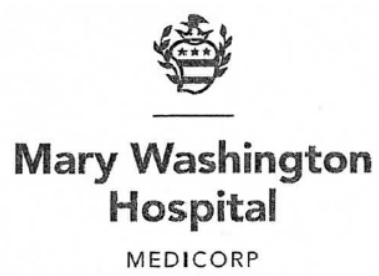

Institutional Review Board

Corey Davis, MSNA, CRNA

9101 Olde Hartley Drive

Glen Allen, VA 23060

Dear Mr. Davis:

The Mary Washington Hospital Institutional Review Board (MWH IRB), which operates in accordance with DHHS regulations for the protection of human research subjects (Title 45 Code of Federal Regulation), the Food and Drug Administration (Title 21 Code of Federal Regulation) and the International Conference of Harmonization (ICH) Good Clinical Practice Guidelines, has reviewed and approved the following protocol:

\section{MWH IRB \# 2010-14 Protocol Anesthesia Recordkeeping: Accuracy of Recall with Computerized} and Manual Entry Recordkeeping

Approval was based on your submission of the following:

- Protocol Submission Form CR 100 dated 6/4/2010

- Recordkeeping Survey Sheet dated 10/26/09

- Recordkeeping Survey - Investigator Sheet dated 10/26/09

- VCU Memo from Lloyd H. Byrd, MS dated 12/21/09

This is notification that you have been granted expedited approval by Rebecca Bigoney, MD, MWH IRB Chair. This action will be reported to all committee members at the next full board meeting of the IRB.

This review was conducted under processes permissible under 45CFR46.110 and 21CFR56.110 category (7) "Research employing survey, interview, oral history, focus group, program evaluation, human factors evaluation, or quality assurance methodologies". Waiver of documentation of informed consent is granted based on 45 CFR 46.117: The only record linking the subject and the research would be the consent document and risk would be potential harm resulting from a breach of confidentiality if the subject demonstrated poor recall accuracy. The research presents no more than minimal risk of harm to subjects and a written consent is not required outside of the research context.

Your IRB approval reference number is \#2010-14. Please include this reference number in all of your future correspondence and reporting to the IRB Office. Your IRB approval expires June 8, 2011.

Please do not hesitate to contact our office at 741-1236 if you have any questions.

Sincerely,

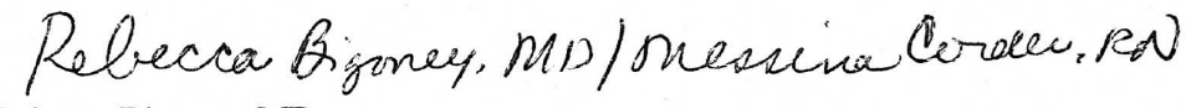

Rebecca Bigoney, MD

Chairman

MWH Institutional Review Board 
VITA 
Thomas Corey Davis was born in Axton, Virginia. He is a 1991 graduate of the Virginia Commonwealth University School of Nursing, and a 1997 graduate of the Virginia Commonwealth University School of Allied Health Professions, Department of Nurse Anesthesia. He has been a practicing Certified Registered Nurse Anesthetist since January of 1998, and is currently the Director of Clinical Education for the VCU School of Allied Health Professions, Department of Nurse Anesthesia. 